Synthesis and Characterization of Nano-to-Meso Scale Ordered Structures in the Si1-xGex Alloy System

\author{
A Dissertation \\ Presented to \\ the faculty of the School of Engineering and Applied Science \\ University of Virginia
}

\author{
in partial fulfillment \\ of the requirements for the degree \\ Doctor of Philosophy \\ by
}

Jatin M. Amatya

December

2016 


\section{APPROVAL SHEET}

The dissertation

is submitted in partial fulfillment of the requirements

for the degree of

Doctor of Philosophy

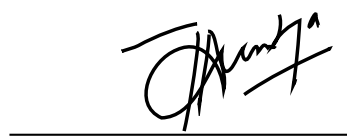

AUTHOR

The dissertation has been read and approved by the examining committee:

\begin{tabular}{c} 
Jerrold A. Floro \\
\hline $\begin{array}{c}\text { Advisor } \\
\text { Petra Reinke }\end{array}$ \\
\hline James M. Howe \\
\hline Stephen McDonnell \\
\hline Joshua Choi
\end{tabular}

Accepted for the School of Engineering and Applied Science:

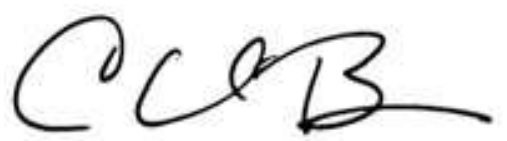

Craig H. Benson, Dean, School of Engineering and Applied Science

December

2016 


\section{Abstract}

This dissertation describes investigations of structural ordering in the Si-Ge material system from nano-to-meso scale in order to modify electronic and thermal transport properties for potential improvement in thermoelectric or nanoelectronics applications.

We first present the synthesis and characterization of mono-layer scale chemically ordered $\mathrm{Si}_{1-\mathrm{x}} \mathrm{Ge}_{\mathrm{x}}$ alloys. Here, metastable ordering to an $\mathrm{L} 1_{1}$-like structure, occurring during heteroepitaxial growth of $\mathrm{Si}_{1-\mathrm{x}} \mathrm{Ge}_{\mathrm{x}}$ thin film alloys on $\mathrm{Si}(001)$ and $\mathrm{Ge}(001)$ substrates, is investigated. A parametric study was performed to study how strain, surface roughness, and growth parameters affect the order parameter during the alloy growth. The order parameter for the alloy films was quantified carefully using x-ray diffraction, taking into account an often-overlooked issue associated with the presence of multiple spatial variants associated with ordering along equivalent $<111>$ directions. The sometimes contradictory roles of strain, extended surface roughness and surface steps in dictating the observed order parameter is discussed.

For the second part, we present our comprehensive investigation on the directed selfassembly of Si-Ge alloy quantum dots (QD) on patterned Si surfaces with variable morphology. Coherently strained $\mathrm{Si}_{1-\mathrm{x}} \mathrm{Ge}_{\mathrm{x}}$ QD self-assemble during epitaxial growth on $\mathrm{Si}(001)$ substrate via the Stranski-Krastanov growth mode, typically with a broad size distribution and spatial disorder. For device applications control over the QD position and size distribution can be realized by templated self-assembly, where a lithographic technique is used to precisely define the nucleation sites and improve the size homogeneity. The templated QDs can then be used as 2D seed "crystals" to propagate the dots into third dimension through subsequent multilayer growth to form quantum dot mesocrystals 
(QDMC). QDMCs have their own synthetic properties related to the mesocrystal structure, such that by manipulating the interdot spacing and the size of the dots of a QDMC structure, we can potentially tune the transport properties. This work develops both an enabling synthesis technology for QDMCs, and generates better fundamental understanding of selfassembly directed by pre-existing modulation of the substrate surface morphology. The multilayer growth is not covered here; however, the research serves as a core foundation to the ongoing efforts in our group for QDMC synthesis along with characterization of the resultant electronic and thermal transport measurements.

For the successful growth of a QDMC structure, the initial underlying 2D template or the substrate pattern morphology plays a critical role. Therefore, the primary challenge was to develop a process and methodology to create highly uniform pit-patterned 2D templates on $\mathrm{Si}(001)$ substrates. Specifically, the challenges of patterning with focused-ion-beam (FIB) and electron beam lithography (EBL) based approach will be discussed. Significant effort was made to avoid extrinsic biases to surface diffusion by residual defectivity and contamination during the pit-patterning of $\mathrm{Si}$ substrates. Growth of $\mathrm{Si}_{1-\mathrm{x}} \mathrm{Ge}_{\mathrm{x}} \mathrm{QDs}$ on these pit-patterned $\operatorname{Si}(001)$ substrates was investigated. Growth of QDs on such patterned substrates is reported to yield precise positioning and improved size homogeneity. However, contradictory results are found regarding QD site selection on patterned substrates. A large body of both theoretical and experimental work has been published to study QD evolution on patterned substrates, but a unified analysis is still lacking regarding the site-selection of QDs on modified substrates. Here, we investigate QD site-selection on a patterned $\mathrm{Si}(001)$ substrate as a function of underlying substrate pattern morphology from pit-in-terrace to quasi-sinusoidal. The pit-in-terrace morphology leads to well-ordered QDs 
centered in the pits over a wide range of pattern wavelengths. However, for a quasisinusoidal morphology, when the pattern wavelength is twice the intrinsic wavelength, QDs suddenly bifurcate and shift to form in every saddle point, with high uniformity in size and site occupancy. Comparison of our results with existing models of QD formation on patterned surfaces will be discussed. Further, we use a relatively unusual approach to growing the $\mathrm{Si}-\mathrm{Ge} \mathrm{QDs}$, in which a conformal layer is grown at low temperature, and then annealed in situ to promote surface diffusion and self-assembly. This provides additional control over the size of the QDs, and turns out to lend additional insights into QD growth kinetics, Ostwald ripening, and morphological transitions in the presence of an underlying surface modulation. 


\section{Acknowledgements}

Firstly, I would like to express my sincere gratitude to my advisor Prof. Jerry Floro for providing me this great opportunity to learn and grow as a research scientist. I would like to thank him for his selfless contributions of time, efforts, guidance and support throughout my graduate carrier. I could not have imagined having a better advisor for my $\mathrm{PhD}$ study. I gratefully acknowledge the funding received from National Science Foundation (NSF) without which this $\mathrm{PhD}$ would not have been possible.

I would like to thank my committee members: Prof. Reinke, Prof. Howe, Prof. McDonnell and Prof. Choi. for their continued guidance and critical questioning of my research.

I would like to thank the Department of Materials Science and Engineering for providing me a great learning environment and especially Kimberly Fitzhugh-Higgins (Kim) for her care and encouragement. I would also like to thank my fellow colleagues, Dr Christoper Petz, Dr. Priya Ghatwai, Greg Stronko, Gopal Ramalingam, Rob Golden, Ehsan Monazami, Junchong Shen, Wade Jensen, Naiming Liu, and Eli Sullivan. I particularly want to thank Dr. Joseph Kassim and Dr. Chris Duska for their selfless support and guidance during my graduate career.

I would like to acknowledge Prof. Arthur Lichtenberger, Alex Lobo, Michael Stogoski, Joseph Beatrice and Jian-Zhong Zhang at the University of Virginia Microfabrication Laboratories and Jonathan Hummel at the University of Maryland for their guidance and assistance during pattern processing. 
Finally, but by no means least, I want to thank my family and especially my wife Dr. Ujuma Shrestha, without whom, I would not have had the courage to embark on this journey in the first place. 


\section{List of Figures}

Figure 1-1: (a) L1 1-like ordered $\mathrm{Si}_{0.5} \mathrm{Ge}_{0.5}$ (RS2) structure, where green and blue atoms represent each chemical species. The view is along the $<321>$ direction, and shows a conventional unit cell of the ordered structure with 64 atoms. The volume shaded red color represents the primitive unit cell. (b) The four equivalent $\{111\}$ planes relative to the (001) surface that dictate the possible spatial variants for RS2 ordering

Figure 1-2: (left) Actual computational cells used in the NEMD simulations. (right)

Thermal conductivity vs. LJ reduced temperature for order parameters from $\operatorname{random}(\eta=0)$ to fully ordered $(\eta=1) .{ }^{17}$

Figure 1-3: (left) $\mathrm{L}_{0}$ and $\mathrm{L}_{2}$ ordered structure in $\mathrm{Cu}-\mathrm{Au}$ system. (right) $\mathrm{Cu}-\mathrm{Au}$ phase diagram showing ordered regions along with electrical resistivity of ordered alloys from experiment (symbols) and theory (lines). ${ }^{18}$

Figure 1-4: (a) Schematic illustration of QDMC (cross-sectional view) along with the unit cell, showing three lattice parameters, and the QD diameter, $\varphi$. (b) Type-II band alignment between the QDs and strained Si matrix.

Figure 1-5: (a) A single SiGe QD embedded in a Si matrix showing the strained Si at top, bottom and edges of the QD due to lattice mismatch forming Type-II band alignment, and (c) Optimized lateral and vertical positioning of QD arrays is predicted to yield coupling of electronic states of QDs resulting in strain-induced high-mobility electron channels

Figure 1-6: The confined electron states (blue, red, green) associated with strain imposed on the surrounding Si matrix by the Ge QDs (yellow). The blue states are of particular interest here as high-mobility conduction channels for electrons. 32

Figure 1-7: (a) Electrical conductivity, (b) power factor and (c) figure of merit - zT; for QDMC structure with doping concentrations of $10^{19} \mathrm{~cm}^{-3}$. For comparison the calculated values of bulk Si are shown for the same doping concentration (dashed gray)

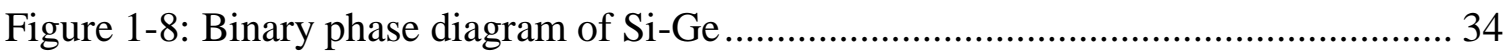

Figure 1-9: Schematic representation of the S-K growth mode ................................. 35 
Figure 1-10: STM images of Ge islands on Si(001): (a) pyramid, (b) dome and (c) superdome. ${ }^{30}$

Figure 1-11: Schematic illustration showing the development of instability during the early stages of $\mathrm{Si}_{1-\mathrm{x}} \mathrm{Ge}_{\mathrm{x}}$ alloy growth. 36

Figure 1-12: STM images of (1) prepyramid island and (b) truncated $\{105\}$ faceted pyramid prior to the formation of a complete $\{105\}$ faceted quasi-pyramidal $3 \mathrm{D}$ islands. ${ }^{37}$.

Figure 1-13: (a) Free energy $\mathrm{E}$ (normalized to the activation energy $\mathrm{E}_{\mathrm{A}}$ for nucleation of (105) faceted huts) as a function of island volume V (relative to critical volume of a hut $\mathrm{V}_{\mathrm{c}}$ ). The solid line shows the energy for (105) facet island and the dashed line shows the evolution path where the sidewall angles grow continuously from $0^{\circ}$ $11^{\circ} .^{36}$ (b) Kinetic phase diagram representing the main growth regimes of $\mathrm{Si}_{1-\mathrm{x}} \mathrm{Ge}_{\mathrm{x}}$ layers. ${ }^{140}$

Figure 1-14: The Matthews and Blakeslee critical thickness plotted against Ge fraction (x). ${ }^{42,141}$.....

Figure 1-15: (a) Measured stress-strain data in SiGe system calculated by Floro et al. ${ }^{47}$ The solid curve (highlighted) is the quadratic fit to the data from which the $\mathrm{M}_{\mathrm{SiGe}}$ can be calculated, (b) Surface energies versus biaxial strain for Ge surface under strain. Highlighted solid line refers to the (001) Ge surface energy under various biaxial strain. ${ }^{48}$ 40

Figure 1-16: $\lambda_{\text {ATGS }}$ plotted against Ge concentration (x), showing our calculated data with experimental and theoretical data from the literature.

Figure 2-1: UHV Hyperthermal MBE setup at UVa showing key components for film synthesis. [A]- MBE chamber, [B]-Load Lock chamber, [C]- RHEED gun, [D]Sputter guns, [E]- Manual gate valve, [F]- Transfer arm.

Figure 2-2: Schematic of a magnetron sputtering process.......................................... 44

Figure 2-3: Probability distribution of sputtered atom energy from the target. ${ }^{54}$ 45

Figure 2-4: (a) 10x10 $\mathrm{m} 2$ AFM height image showing patterned region prior to the insertion to MBE. (b) 10x10 $\mu \mathrm{m} 2$ AFM height image of the same area as shown in (a) post $800{ }^{\circ} \mathrm{C}$ desorb cycle in $\mathrm{MBE}$ - no Si buffer deposited. 
Figure 2-5: Schematic of the RHEED setup in the MBE chamber showing a RHEED pattern recorded from a $\mathrm{Si}(001)(2 \times 1)$ surface along a $<110>$ direction.

Figure 2-6: RHEED patterns of Si (001) along <110>: (a) diffuse 2D pattern prior to desorb cycle, (b) first order and (c) second order Laue spots indicating a (2x1) surface reconstruction of $\mathrm{Si},(\mathrm{d})$ atomically rough $3 \mathrm{D}$ pattern, showing diffraction through 3D QDs.

Figure 2-7: Layout of an atomic force microscopy (NTMDT solver pro AFM manual) . 50 Figure 2-8: AFM micrograph of Si (001) showing the surface morphology post (a) $50 \mathrm{~nm}$ of Si buffer layer growth at $700{ }^{\circ} \mathrm{C}$, and (b) $3 \mathrm{~nm}$ of $\mathrm{Si}_{0.5} \mathrm{Ge}_{0.5}$ alloy growth at 650 ${ }^{\circ} \mathrm{C}$

Figure 2-9: Schematic of the Rigaku SmartLab x-ray diffractometer. ${ }^{62}$........................ 52

Figure 2-10: (a) Bragg peaks for an epitaxial film (single crystal) (b) Diffraction rings . 52 Figure 2-11: Reciprocal space map (RSM) of (a) 004 peak and (b) 224 peak for $300 \mathrm{~nm}$ thick $\mathrm{Si}_{0.5} \mathrm{Ge}_{0.5}$ alloy grown on $\mathrm{Si}(001)$ substrate. $\mathrm{Q}_{\mathrm{x}}$ and $\mathrm{Q}_{\mathrm{z}}$ are the reciprocal space units along <110> and <001> respectively. ( $Q_{z}$ for (224) scan is along <224> however it has been normalized along <001> for comparison with (004) RSM scan)

Figure 2-12: Schematics of XRD scanning geometry and direction. 54

Figure 2-13: $\omega$-2 $\theta$ scan of (004) and (224) peak for $100 \mathrm{~nm}$ thick $\mathrm{Si}_{0.5} \mathrm{Ge}_{0.5}$ alloy deposited on $\mathrm{Si}(001)$ substrate: (a), (b) and Ge(001) substrate: (c), (d).

Figure 2-14: X-ray reflectivity curve of: (a) Au film on Si substrate with different thickness, (b) $\mathrm{Au}, \mathrm{Cu}$ and $\mathrm{SiO}_{2}$ film on $\mathrm{Si}$ substrate (film thickness is $20 \mathrm{~nm}$ ), (c) $\mathrm{Si}$ substrate with two different values of surface roughness, and (d) Si substrate with different interface roughness (film thickness is $20 \mathrm{~nm})^{72}$ 57

Figure 2-15: XRR data for nominal 100nm thick $\mathrm{Si}_{0.5} \mathrm{Ge}_{0.5} / \mathrm{Si}(001)$ sample. The reflectivity curve obtained from sample was analyzed with Rigaku software simulator; extracted results are listed in the table. 58

Figure 2-16: Comparison of 1/2(111) superlattice peak of $300 \mathrm{~nm}$ thick $\mathrm{Si}_{0.5} \mathrm{Ge}_{0.5} / \mathrm{Si}(001)$ grown at various temperature with (111) fundamental alloy peak for order parameter calculation. 59 
Figure 2-17: Outline of EBL patterning process to form nanoscale pattern with a positivetone resist layer. 60

Figure 2-18: (a) Dot-pattern generated with AutoCAD for e-beam exposure, (b) optical image of the patterned region after developing the patterns, (c) SEM image of one of the patterned region, and (d) a series of dot/pixel exposed at increasing dose from bottom to top showing the ability to control the feature size with dose control....... 61

Figure 2-19: (a) Raith-50 at UVa was not optimized to perform patterning with pitch $\leq$ $500 \mathrm{~nm}$ where patterns with lower pitch were non-uniform. (b)Pattern pitch 75-200 nm; Raith-e-line was able to perform high resolution patterning with better uniformity and precision 62

Figure 2-20: A schematic diagram of electron-sample interaction. ${ }^{73}$ 63

Figure 2-21: TEM images of a $500 \mathrm{~nm}$ thick $\mathrm{Si}_{0.5} \mathrm{Ge}_{0.5} / \mathrm{Si}$ sample (a) BF image, (c) STEM image: Ge having a greater $\mathrm{Z}$ than $\mathrm{Si}$ therefore $\mathrm{SiGe}$ alloy appears as the lighter intensity due to increased high angle scattering, and (c) HRTEM image along with FFT image showing the Si lattice along a $<110>$ ZA.

Figure 2-22: SEM images of (a) patterned region from where a TEM cross-section is required, (b) $50 \mu \mathrm{m}$ wide carbon strap is deposited to protect the patterned region on the surface, (c)-(e) sequential milling steps into the Si substrate to expose a sample for lift-out, and (f) thinned sample mounted to half grid for TEM.

Figure 3-1: Proposed rhombohedral structure (RS) models for the ordered structure in $\mathrm{Si}_{1}$ ${ }_{x} G_{x}$ alloys: (a) RS1 model, (b) RS2 model and (c) RS3 model. View for all the ordered unit cell is along $<110>. \alpha, \beta$ and $\gamma, \delta$ represent Ge-rich and Si-rich sites, respectively. ${ }^{7}$

Figure 3-2: (a) Cross-sectional view of a 2x1 dimer surface along a $\langle 110\rangle$. Surface dimers result in the strain underneath the surface resulting in sites under compression favoring Si occupancy, black circles, and sites under tension favoring Ge occupancy, white circles. (b) Proposed growth model by LeGoues, et al., where growth occurs by the motion of double steps in order to permit large domains along a single $\langle 111>$. 5 69 
Figure 3-3: Proposed growth model by Jesson, et al., with growth-induced compositional ordering by kink flow growth at $\mathrm{D}_{\mathrm{B}}$ steps. (a) Plan-view of a $2 \times 1$ dimer surface with kink where, $\mathrm{A}$ and $\mathrm{B}$ are surface dimers, and $\mathrm{C}$ to $\mathrm{E}$ represent rebonded edge atoms at the step edge. (b) to (e) shows how the growth takes place by the Ge-pump mechanism resulting in the ordered structure where $\alpha, \beta$ and $\gamma, \delta$ represent Ge-rich and Si-rich sites, respectively. White circles represent Ge atoms and black circles represent $\mathrm{Si}$ atoms. ${ }^{15}$

Figure 3-4: Surface stress induced ordering mechanism proposed by LeGoues, et al., and revised by Araki, et al., showing ML by ML growth process of the ordering 71

Figure 3-5: $\omega$-2 $\theta$ scan for $100 \mathrm{~nm}$ thick $\mathrm{Si}_{0.5} \mathrm{Ge}_{0.5} / \mathrm{Ge}(001)$, (a) (111) azimuth scan showing significant alloy peak overlapping with the substrate peak, and (b) (333) azimuth scan showing distinct alloy peak resulting in lesser peak-fitting errors. .... 74

Figure 3-6: Computed structure factor amplitude comparison with that of the RS2 model by Tischler, et al., for the half-order superlattice reflections. 76

Figure 3-7: Order parameter for $300 \mathrm{~nm}$ thick $\mathrm{SiGe/Si}$ samples grown at various temperatures. The $400^{\circ} \mathrm{C}$ sample was grown several times to ensure repeatability. 78 Figure 3-8: (a) Effective order parameter $\left(\mathrm{S}_{\mathrm{eff}}\right)$ plotted against growth temperature for $100 \mathrm{~nm} \mathrm{Si}_{0.5} \mathrm{Ge}_{0.5}$ on $3^{\circ}$ miscut $\mathrm{Si}(001)$ substrate, and (b) True order parameter for $300 \mathrm{~nm}$ thick $\mathrm{Si}_{0.5} \mathrm{Ge}_{0.5} / \mathrm{Si}(001)$ and $100 \mathrm{~nm}$ thick $\mathrm{Si}_{0.5} \mathrm{Ge}_{0.5} / \mathrm{Ge}(001)$. 79

Figure 3-9: Order parameter vs Deposition rate for $100 \mathrm{~nm}$ thick $\mathrm{Si}_{0.5} \mathrm{Ge}_{0.5} / \mathrm{Si}(001) \ldots \ldots .80$

Figure 3-10: (a) TEM bright field cross-sectional image of a $\mathrm{Si}_{0.5} \mathrm{Ge}_{0.5} 500 \mathrm{~nm}$ alloy on $\mathrm{Si}(001)$ low-miscut substrate (b) SAD pattern along [-1-10], and (c) SAD pattern along [-3-10] zone axis showing half-order superlattice diffraction spots. 80

Figure 3-11: DF image taken by placing an OA over the corresponding superlattice peaks as labelled with [-3-10] ZA. The BF image of the sample is shown in Figure 3-10. 82 Figure 3-12: (a) HRSTEM image for $500 \mathrm{~nm}$ thick $\mathrm{Si}_{0.5} \mathrm{Ge}_{0.5} / \mathrm{Ge}(001)$ along [110] ZA, (b) FFT image taken from the HRSTEM image, (c) Domain 1 (red) created by performing reverse FFT and taking $1 / 2(-11-1)$ and $1 / 2(1-11)$ superlattice spots, (d) Domain 2 (green) created by performing reverse FFT and taking $1 / 2(-111)$ and 1/2(1-11) superlattice spots, (e) superimposed image of (c) and (d) 83 
Figure 3-13: Miscut changes the step population on the growth surface; $S_{N}$ is the surface normal (b) Effective order parameter of all four ordered domains, for 100nm thick $\mathrm{Si}_{0.5} \mathrm{Ge}_{0.5}$ alloy on $3^{\circ}$ miscut $\mathrm{Si}$ substrates.

Figure 3-14: Order parameter vs thickness for samples grown on low-miscut (miscut of $\left.<0.1^{\circ}\right) \mathrm{Si}(001)$ and $\mathrm{Ge}(001)$ substrate. 85

Figure 3-15: AFM micrographs and local area slope images showing the morphology of $\mathrm{Si}_{0.5} \mathrm{Ge}_{0.5}$ (a) $100 \mathrm{~nm}$, (b) $300 \mathrm{~nm}$, (c) $500 \mathrm{~nm}$ thick alloy films grown on $\mathrm{Ge}(001)$ substrate and (d) $100 \mathrm{~nm}$, (e) $300 \mathrm{~nm}$, (f) $500 \mathrm{~nm}$ thick alloys on $\mathrm{Si}(001)$ substrate. (g) Color bar indicates the local slope in degrees.

Figure 3-16: (a) Order parameter vs surface roughness, (b) order parameter vs residual strain; labels 1,3 and 5 refer to $100 \mathrm{~nm}, 300 \mathrm{~nm}$ and $500 \mathrm{~nm}$ thick alloy, respectively. A residual strain of 50\% means that half the initial mismatch strain has been relaxed.

Figure 3-17: Order parameter for $300 \mathrm{~nm}$ thick $\mathrm{SiGe/Si}$ samples grown at various temperatures with two different MBE setups. 88

Figure 4-1: AFM image showing $\mathrm{Si}_{0.5} \mathrm{Ge}_{0.5}$ QDs nucleating randomly on a $\mathrm{Si}(001)$ substrate: (a) 3D perspective view, (b) topography image, and (c) local area slope image outlining the facets of quasi-pyramidal and dome morphology.

Figure 4-2: (a) Single layer of S-K Ge QDs, capped with Si, but randomly oriented inplane. (b) A second layer aligns to the first via propagated strain fields on the Si cap surface. (c) Repeating the Ge/Si growth sequence leads to a QDSL. (d) If the first layer of Ge QDs are made to be 2D periodic, then subsequent Ge/Si layering leads to a QDMC. 97

Figure 4-3: Various classes of ordered nanostructures: (a) periodically faceted surfaces; (b) surface structures of planar domains; (c) ordered array of coherently strained islands; (d) cross-sectional scheme of a multisheet array of 2D islands; (e) crosssectional scheme of a multisheet array of 3D islands (QDMC). ${ }^{7}$

Figure 4-4: (a) QDs ordering along <100> near the edges of a lithographically defined $\mathrm{Si}(001)$ plateau $^{16}$, (b) QDs aligned along a $<110>$ oriented ridges of the Si strip mesas $^{17}$, (c) trench formation on the substrate resulting in the strain field modulation 
to direct QD formation on a flat top surface ${ }^{19}$, (d) QDs nucleating inside the pits of a pit-patterned substrate yielding highly ordered dots ${ }^{34}$. 98

Figure 4-5: Schematic of a pit profile with top (t), bottom (b) and sidewall (s). Due to the curvature, the chemical potential is minima at the pit-bottom $\left(\mu_{\mathrm{t}}>\mu_{\mathrm{s}}>\mu_{\mathrm{b}}\right){ }^{35}$. 99

Figure 4-6: AFM images in derivative mode showing the results for $3 \mathrm{ML}$ of Ge deposited on a pit-patterned $\mathrm{Si}(001)$ substrate at $700{ }^{\circ} \mathrm{C}$ with various pitch $\left(\lambda_{\mathrm{p}}\right)$ ranging $425 \mathrm{~nm}-3400 \mathrm{~nm}$. 101

Figure 4-7: Local area slope images for a single pit comparing the position/ localization of QDs relative to the pit-sidewall angle $\left(\alpha_{\mathrm{pit}}\right)$. QDs were observed to localize at the edges for $\alpha_{\text {pit }}>30^{\circ} .^{14}$ 102

Figure 4-8: (a) Elastic energy density ( $\rho$ ) vs pit inclination angle $(\alpha)$ plot, $\rho$ is normalized to the energy density of Ge dome on a flat surface. Elastic energy maps for domes with (a) $\alpha=0^{\circ}$ (flat surface), (b) $\alpha=20^{\circ}$, and (c) $\alpha=60^{\circ} .{ }^{23}$ 102

Figure 4-9: (a) 1x1 $\mu \mathrm{m} 2$ AFM image of the top layer of QDMC with lateral period of 49 nm. (b) Cross-section STEM image of the sample shown in (a) 103

Figure 4-10: AFM data for $50 \mathrm{~nm}$ pitch pattern showing (a) Ge QDs and (b) same region of Si surface after Ge was etched off. (c) AFM linescans from the Ge QDs (blue) and Si surface (black) after etching Ge QDs. 104

Figure 4-11: $2.5 \times 2.5 \mu \mathrm{m}^{2}$ AFM image showing the results for $1.4 \mathrm{~nm}$ of Ge deposited on patterned $\mathrm{Si}(001)$ substrate at different growth temperatures $\left(\mathrm{T}_{\text {growth }}\right)$. (a) $\mathrm{T}_{\text {growth }}=$ $750{ }^{\circ} \mathrm{C}$ with pitch $=150 \mathrm{~nm}$ (inset shows the higher magnification image of 3 QDs localized on crown region), (b) $\mathrm{T}_{\text {growth }}=750{ }^{\circ} \mathrm{C}$ with pitch $=350 \mathrm{~nm}$, and (c) $\mathrm{T}_{\text {growth }}$ $=550{ }^{\circ} \mathrm{C}$ with pitch $=150 \mathrm{~nm}$ showing QDs nucleating only in the pits. ${ }^{9}$ 105

Figure 4-12: (a) $\lambda_{\eta}$ is the artificial wavelength imposed by pattern and $\lambda_{\text {ATGS }}$ is the intrinsic wavelength. (b) Phase space for growth location of Ge QDs on a quasisinusoidal pattern, $\mathrm{H}$ is the film thickness and $\mathrm{H}_{\mathrm{c}}$ is the wetting layer thickness... 106

Figure 5-1: Topographic AFM images of 30kV energy Ga+ ion patterns for various ion dose with 8ML Ge (no Si buffer). (a) 20K ions/site with $100 \mathrm{~nm}$ pitch, (b) $10 \mathrm{~K}$ ions/site with $100 \mathrm{~nm}$ pitch, (c) $5 \mathrm{~K}$ ions/site with $100 \mathrm{~nm}$ pitch, (d) $3 \mathrm{~K}$ ions/site with $100 \mathrm{~nm}$ pitch, (e) $2 \mathrm{~K}$ ions/site with $100 \mathrm{~nm}$ pitch, (f) off-pattern region 109 
Figure 5-2: Topographic AFM images of 30kV energy Ga+ ion patterns for various ion dose. (a) $5 \mathrm{~K}$ ions/site with $50 \mathrm{~nm}$ pitch and $20 \mathrm{~K}$ ions/site with $100 \mathrm{~nm}$ pitch, (b) $10 \mathrm{~K}$ ions/site with $100 \mathrm{~nm}$ pitch, (c) $5 \mathrm{~K}$ ions/site with $100 \mathrm{~nm}$ pitch, (d) $3 \mathrm{~K}$ ions/site with $100 \mathrm{~nm}$ pitch, (e) $2 \mathrm{~K}$ ions/site with $100 \mathrm{~nm}$ pitch, (f) $1 \mathrm{~K}$ ions/site with $100 \mathrm{~nm}$ pitch

Figure 5-3: Topographic AFM images of $8 \mathrm{kV}$ energy $\mathrm{Ga}+$ ion patterns with (a) $5 \mathrm{~K}$ ions/site with $50 \mathrm{~nm}$ pitch and $20 \mathrm{~K}$ ions/site with $100 \mathrm{~nm}$ pitch; (b) $10 \mathrm{~K}$ ions/site with $100 \mathrm{~nm}$ pitch and 5K ions/site with 100nm pitch; (c) 5K ions/site with $100 \mathrm{~nm}$ pitch, $3 \mathrm{~K}$ ions/site with $100 \mathrm{~nm}$ pitch and $3 \mathrm{~K}$ ions/site with $100 \mathrm{~nm}$ pitch 111

Figure 5-4: Topographic AFM images of $30 \mathrm{kV}$ energy Ga+ ion patterns with (a) $15 \mathrm{~nm} \mathrm{Si}$ buffer at $700{ }^{\circ} \mathrm{C}$ with $6 \mathrm{ML} \mathrm{Ge}$ at $600{ }^{\circ} \mathrm{C}$; (b) $10 \mathrm{~nm}$ Si buffer at $600{ }^{\circ} \mathrm{C}$ with $6 \mathrm{ML}$ Ge at $600{ }^{\circ} \mathrm{C}$ 112

Figure 5-5: Schematic for the workflow used for EBL based patterning approach optimization. 113

Figure 5-6: 950K PMMA-A resists spin curve, solids: $2 \%-7 \%$ in Anisole. ${ }^{10}$ 115

Figure 5-7: Forward and backscattering of incident electrons during resist exposure. .. 116 Figure 5-8: $500 \mathrm{~nm}$ pitch pattern exposed using Raith-50 at a constant dose with spot size 2 and beam energy of (a) $10 \mathrm{kV}$, (b) $15 \mathrm{kV}$, (c) $20 \mathrm{kV}$ and (d) $25 \mathrm{kV}$. At lower and higher beam energy, the beam broadening effect is prominent. 117

Figure 5-9: (a) Dot-pattern generated with Elphy Quantum Raith software for e-beam exposure showing (b) constant dose test field and dose variation test field along with (c) an optical image of the patterned region after development stage 118

Figure 5-10: Dose variation test field showing AFM micrograph of 75 and $100 \mathrm{~nm}$ pattern along with calibrated color dose range map comparing three distinct regions.

Figure 5-11: Plot of Dot diameter or pit opening vs. dot dose. Blue dots are the actual data and red dashed line is the fitted curve. Dot diameter is the pit opening width.

Figure 5-12: $2 \times 2 \mu \mathrm{m}^{2}$ AFM images showing the effect of dose change at various pitch. Dose (d) is increasing from left to right and is given in units of $10^{-3} \mathrm{pC}$. 121 
Figure 5-13: Plot of dot dose vs pattern pitch. The symbols represent the actual data with boundaries separating the three distinct regions observed experimentally during exposure - (1) under-dose region: result of insufficient clearance of the resist, (2) acceptable/ideal-dose region, and (3) over-dose region: excessive dose or exposure of pattern resulting in pattern degradation.

Figure 5-14: (a) Polymer sub-unit of PMMA, (b) fragmentation of polymer chain during EBL exposure, and (c) Spatial distribution of volume fraction of small fragments (less than 10 monomers) within the resist during single point exposure. ${ }^{2}$. 123

Figure 5-15: 1.5x1.5 $\mu \mathrm{m}^{2}$ AFM image with $50 \mathrm{~nm}$ pitch. The patterns were developed at room temperature (RT) and at $-10{ }^{\circ} \mathrm{C}$ with a development time of $\sim 10 \mathrm{sec}$. The pattern resolution and contrast is clearly enhanced by the low temperature development process. The line scan is taken from the $-10^{\circ} \mathrm{C}$ development sample.

Figure 5-16: (a) anisotropic etching, and (b) isotropic etching of Si during RIE.......... 126

Figure 5-17: cross-sectional SEM image of $50 \mathrm{~nm}$ wide features etched with $\mathrm{SF}_{6}$ (12 $\mathrm{sccm})$ and $\mathrm{C}_{4} \mathrm{~F}_{8}(27 \mathrm{sccm})$ chemistry at $10{ }^{\circ} \mathrm{C}, 10 \mathrm{~m}$ Torr pressure, $750 \mathrm{~W}$ ICP power and 20 W RF power. (source: Oxford PlasmaLab 100 RIE manual)

Figure 5-18: $2 \times 2 \mu \mathrm{m}^{2}$ AFM images showing $150 \mathrm{~nm}$ pattern pitch; (a) after the development stage (before RIE), (b) post RIE and resist removal stage, and (c) line scan along <110> comparing the pattern morphology showing square-wave like patterns post RIE. 128

Figure 6-1: Time vs growth temperature plot of the deposition cycle in MBE divided into 4 distinct regions. $t_{\text {anneal }}$ is the annealing time. 131

Figure 6-2: $2 \times 2 \mu \mathrm{m}^{2}$ AFM images of $150 \mathrm{~nm}$ pitch pattern: (a) after RIE and resist cleaning, (b) after $50 \mathrm{~nm}$ of Si buffer growth at $450{ }^{\circ} \mathrm{C}$, and (c) line scan along $<110>$ showing the transition of rough and square-wave-like pattern to smooth Gaussian-like pit pattern profile 132

Figure 6-3: $2 \times 2 \mu \mathrm{m}^{2}$ AFM images of $100 \mathrm{~nm}$ pitch pattern (a) after RIE, and (b) after 50 $\mathrm{nm}$ Si buffer deposited at $550{ }^{\circ} \mathrm{C}$. 133 
Figure 6-4: AFM images along with the local-area slope image after $50 \mathrm{~nm}$ of Si buffer deposition for pattern with pitch (a) $100 \mathrm{~nm}$, (b) $150 \mathrm{~nm}$, and (c) $200 \mathrm{~nm}$............ 133

Figure 6-5: (a) $5 \times 5 \mathrm{~m}^{2}$ AFM image showing the disappearance of pits at the lowest dose region where the pits have narrow opening and shallow depth, (b) local slope image of the same AFM image showing the decreasing pit-sidewall slope with decreasing dose.

Figure 6-6: (a) Pattern with $\lambda_{\mathrm{p}}=150 \mathrm{~nm}$ after Si buffer growth. The long linescan along [110] reveals the gradual evolution from pit-in-terrace to quasi-sinusoidal. The inset defines the locations: (1) pit bottom, (2) crown region and (3) saddle region. (b) Line scan along [110] comparing pit-in-terrace vs sinusoidal-like profile. 135

Figure 6-7: Comparison of the AFM images with their corresponding line scans along $<110>$ and $<100>$ for the deposition of $1.3 \mathrm{~nm}$ of $\mathrm{Si}_{0.5} \mathrm{Ge}_{0.5}$ on patterns with pitch (a) $100 \mathrm{~nm}$, (b) $150 \mathrm{~nm}$ and (c) 200nm. For patterns with same pitch, the scans were taken from three different samples varying $t_{\text {anneal, }}$ but in the same dose region such that the pit morphology is nominally identical prior to annealing. $\left(2 \times 10^{-3} \mathrm{pC}, 2.5 \times 10^{-}\right.$ ${ }^{3} \mathrm{pC}$ and $\left.4.5 \times 10^{-3} \mathrm{pC}\right)$..... 138

Figure 6-8: Comparison of volume distribution of QDs for the deposition of $1.3 \mathrm{~nm}$ of $\mathrm{Si}_{0.5} \mathrm{Ge}_{0.5}$ in absolute (left) and normalized (right) units, at various $\mathrm{t}_{\text {anneal }}$ for patterns with pitch (a) $100 \mathrm{~nm}$, (b) $150 \mathrm{~nm}$, and (c) 200nm. 141

Figure 6-9: Schematic diagram of the absolute collection area and effective collection area per unit pit in terms of $\lambda_{p}$ and surface diffusion length $\left(d_{1}\right)$. If $d_{l}>\lambda_{p} / 2$ or comparable to $\lambda_{\mathrm{p}}$, ripening of QDs will dominate as the annealing time progresses.

Figure 6-10: Effective collection area for four individual neighboring pits with pattern pitch of (a) $100 \mathrm{~nm}$, (b) $150 \mathrm{~nm}$, and (c) $200 \mathrm{~nm}$. The dimensions in the schematics are in scale for comparison where $d_{1}$ has been scaled to match $90 \mathrm{~nm}$ (refer section 6.8 for our diffusion length calculations). 143

Figure 6-11: (a) Plot of mean volume vs anneal time at various pitch for $1.3 \mathrm{~nm}$ of $\mathrm{Si}_{0.5} \mathrm{Ge}_{0.5}$ alloy. The plot is subdivided into four distinct regions outlining the growth kinetics. (b) Plot of normalized FWHM vs anneal time 145 
Figure 6-12: Comparison of AFM topography scans of quasi-sinusoidal pattern region at various $t_{\text {anneal }}$ for $1.3 \mathrm{~nm} \mathrm{Si}{ }_{0.5} \mathrm{Ge}_{0.5}$ deposited on patterns with pitch (a) $150 \mathrm{~nm}$, (b) $175 \mathrm{~nm}$, and (c) $200 \mathrm{~nm}$.

Figure 6-13: $2 \times 2 \mu \mathrm{m}^{2}$ AFM micrographs along with local slope images and 3D perspective view of $\mathrm{Si}_{0.5} \mathrm{Ge}_{0.5}$ alloy deposited at $450{ }^{\circ} \mathrm{C}$ and then annealed at $650{ }^{\circ} \mathrm{C}$ for $\mathrm{t}_{\text {anneal }}=5 \mathrm{~min}$ with alloy thickness of (a) $1.3 \mathrm{~nm}$, (b) $1.6 \mathrm{~nm}$, (c) $2 \mathrm{~nm}$, (d) 2.6 $\mathrm{nm}$, and (e) $3 \mathrm{~nm}$. 149

Figure 6-14: Comparison of AFM topography images for $1.3 \mathrm{~nm}$ and $2.6 \mathrm{~nm}$ of $\mathrm{Si}_{0.5} \mathrm{Ge}_{0.5}$ alloy with their corresponding local area slope image and 3D perspective image for patterns with pitch (a) $100 \mathrm{~nm}$, (b) $150 \mathrm{~nm}$, and (c) $200 \mathrm{~nm} .\left(2 \times 10^{-3} \mathrm{pC}, 2.5 \times 10^{-3} \mathrm{pC}\right.$ and $4.5 \times 10^{-3} \mathrm{pC}$ for pitch 100,150 and $200 \mathrm{~nm}$ respectively) 150

Figure 6-15: (a) 3D perspective image, (b) AFM topography image, and (c) local area slope image comparing patterned and non-patterned region for $2.6 \mathrm{~nm}$ thick $\mathrm{Si}_{0.5} \mathrm{Ge}_{0.5}$ alloy annealed at $650{ }^{\circ} \mathrm{C}$ for $5 \mathrm{~min}$. 151

Figure 6-16: Comparison of volume distribution of QDs for the deposition of $1.3 \mathrm{~nm} v \mathrm{~s}$ $1.6 \mathrm{~nm}$ of $\mathrm{Si}_{0.5} \mathrm{Ge}_{0.5}$ in absolute and normalized scale for pattern with pitch (a) 100 $\mathrm{nm}$, (b) $150 \mathrm{~nm}$, and (c) $200 \mathrm{~nm}$. 153

Figure 6-17: AFM topography scans of quasi-sinusoidal pattern region at $650{ }^{\circ} \mathrm{C}$ with $\mathrm{t}_{\text {anneal }}=5 \mathrm{~min}$, comparing $1.3 \mathrm{~nm}$ vs $2.6 \mathrm{~nm}$ thick $\mathrm{Si}_{0.5} \mathrm{Ge}_{0.5}$ alloy deposited on patterns with pitch (a) $150 \mathrm{~nm}$, (b) $175 \mathrm{~nm}$, and (c) $200 \mathrm{~nm}$. 154

Figure 6-18: Comparison of AFM image (left) along with volume distribution (right) of QDs for the deposition of $1.3 \mathrm{~nm}$ thick $\mathrm{Si}_{0.5} \mathrm{Ge}_{0.5}$ alloy annealed at $650{ }^{\circ} \mathrm{C}$ with $\mathrm{t}_{\text {anneal }}$ $=5 \mathrm{~min}$ for patterns with pitch (a) $100 \mathrm{~nm}$, (b) $150 \mathrm{~nm}$, and (c) 200nm. 156

Figure 6-19: AFM micrographs comparing the effect of pattern orientation for the pit-interrace and quasi-sinusoidal patterned region with $\lambda_{\mathrm{p}}=150 \mathrm{~nm}$, (a) pattern oriented along $\langle 110\rangle$, and (b) pattern oriented along $\langle 100\rangle$. 157

Figure 6-20: AFM 3D perspective image along with topography and local area slope image showing the comparative results for $2.6 \mathrm{~nm}$ thick $\mathrm{Si}_{0.5} \mathrm{Ge}_{0.5}$ alloy growth on $\lambda_{\mathrm{p}}$ $=200 \mathrm{~nm}$ region with varying pit-opening size $-\mathrm{W}\left(\mathrm{t}_{\text {anneal }}=5 \mathrm{~min}\right.$ at $\left.650{ }^{\circ} \mathrm{C}\right) \ldots .159$

Figure 6-21: Schematics of patterned regions showing the diffusion length parameters along with pattern pitch $\left(\lambda_{\mathrm{p}}\right)$ and pit-opening $(\mathrm{W})$. 160 
Figure 6-22: Schematics of the growth conditions showing surface diffusion kinetics for (a) adatom species under a constant deposition flux J, and (b) adatom species during annealing post deposition at lower temperature. ESDNo anneal is the energy barrier for surface adatoms to diffuse and ESDAnneal is the energy barrier for the surface adatoms to diffuse post deposition during anneal. 162

Figure 6-23: AFM micrographs highlighting the transition regions in patterns with $\lambda_{\mathrm{p}}=$

(a) $100 \mathrm{~nm}$, (b) $125 \mathrm{~nm}$, (c) $150 \mathrm{~nm}$, (d) $175 \mathrm{~nm}$, and (e) $200 \mathrm{~nm}$. (1.3 nm thick $\mathrm{Si}_{0.5} \mathrm{Ge}_{0.5}$ alloy with $\mathrm{t}_{\text {anneal }}=5 \mathrm{~min}$ at $650{ }^{\circ} \mathrm{C}$ ) 163

Figure 6-24: AFM micrographs from $\lambda \mathrm{p}=150 \mathrm{~nm}$ pattern, showing (a) saddle nucleation, and (b) pit nucleation. Both topography (left) and local slope (right) images are shown. In (a), the inset shows a close-up of four QDs on the saddle points surrounding a pit. All the QDs are $\{105\}$ faceted. (c) and (d) are 3D perspective image of (a) and (b) AFM topography images respectively. (e) and (f) are Line scan comparisons along $\langle 110\rangle$ and $<100\rangle$ respectively. (for line scan comparison the AFM images has been normalized to crown region) 164

Figure 6-25: Maps of the observed QD site preference as a function of the pattern pitch $\left(\lambda_{\mathrm{p}}\right)$ and the pit opening width $(\mathrm{W})$ for $1.3 \mathrm{~nm} \mathrm{Si}_{0.5} \mathrm{Ge}_{0.5}$ alloy growth with $\mathrm{t}_{\text {anneal }}=5$ min at $650{ }^{\circ} \mathrm{C}$ in (a) absolute scale and (b) normalized scale. In (b), the transition region $(\mathrm{TR})$ is indicated.

Figure A1-1: Deposition rates for (a) Si sputter target and (c) Ge sputter target. The calibration was performed at a chamber pressure of 4.75 mTorr of Ar. 186

Figure A1-2: (a) Manipulator assembly installed with a 2 inch wafer handling system along with substrate heating assembly, (b) tungsten heating filament coiled around ceramic beads which is about 2 inch in diameter to heat the substrate uniformly, and (c) heating filament under operation at UHV condition. 187

Figure A1-3: Temperature vs. Filament current plot 188

Figure A3-1: Slope distribution plots of $\mathrm{Si}_{0.5} \mathrm{Ge}_{0.5}$ alloys grown on $\mathrm{Ge}(001)$ with thickness (a) $100 \mathrm{~nm}$, (b) $300 \mathrm{~nm}$, and (c) $500 \mathrm{~nm}$. 192 
Figure A3-2: Slope distribution plots of $\mathrm{Si}_{0.5} \mathrm{Ge}_{0.5}$ alloys grown on $\mathrm{Si}(001)$ with thickness

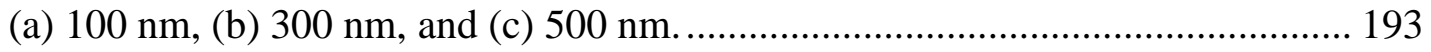

Figure A4-1: Probability plots comparing to the normal distribution reference line for three of the datasets discussed in chapter $6.100 \mathrm{~nm}$ pitch pattern with a population of 300 points and anneal time of (a) $2 \mathrm{~min} 30 \mathrm{sec}$, (b) $5 \mathrm{~min}$, and (c) $10 \mathrm{~min} \ldots \ldots . . .195$ 


\section{List of Terms and Symbols}

AFM: Atomic force microscopy

$\mathrm{a}_{\mathrm{Ge}}$ : Lattice constant of $\mathrm{Ge}$

asi: Lattice constant of $\mathrm{Si}$

asiGe: Lattice constant of SiGe alloy

ATGS: Asaro-Tiller-Grinfeld-Srolovitz

BZ: Brillouin zone

$\mathrm{d}_{\mathrm{e}-\mathrm{e}}$ : Edge to edge distance between pits

$\mathrm{d}_{1}$ : Surface diffusion length of adatoms

EBL: Electron beam lithography

FCC: Face centered cubic

FIB: Focused-ion beam

IPA: Isopropyl alcohol

L: Lorentz factor

MBE: Molecular beam epitaxy

MIBK: Methyl isobutyl ketone

ML: monolayer

NEMD: Non-equilibrium molecular dynamics

PMMA: Poly-methyl methacrylate

QD: Quantum dot

QDMC: Quantum dot mesocrystal

RHEED: Reflection high-energy electron diffraction

RS: Rhombohedral structure

RT: Room temperature

S: Seebeck coefficient

S-K: Stranski-Krastanov

TCE: Trichloroethylene

UV: Ultraviolet

W: Pit-opening width

WL: Wetting layer 
XRD: X-ray diffraction

XRR: X-ray reflectivity

zT: Thermoelectric figure of merit

$\kappa:$ Thermal conductivity

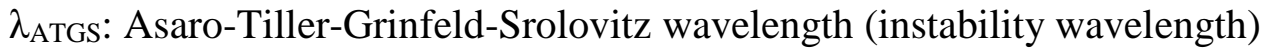

$\lambda_{\mathrm{p}}$ : Pattern pitch 


\section{Table of Contents}

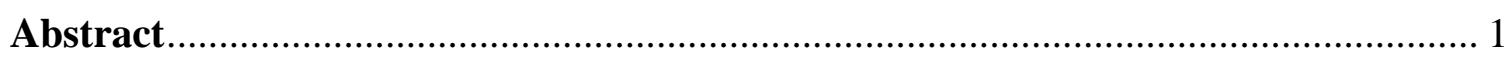

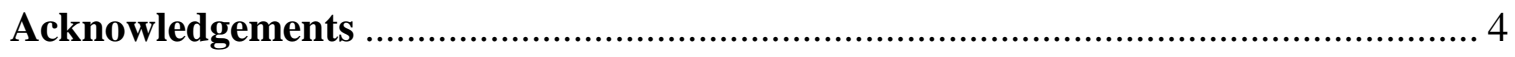

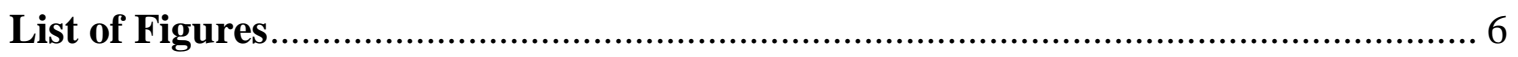

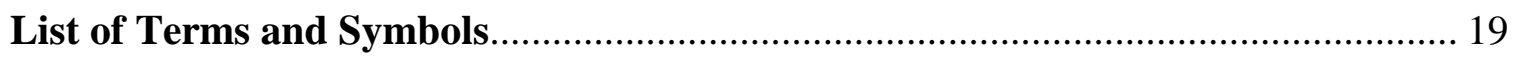

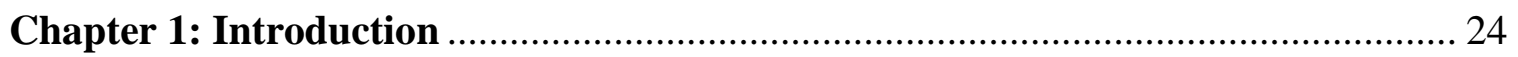

$1.1 \quad$ Background and Motivation ...................................................................... 24

1.1.1 Nano-scale ordered structure in $\mathrm{Si}_{1-\mathrm{x}} \mathrm{Ge}_{\mathrm{x}}$ alloys....................................... 26

1.1.2 Meso-scale ordered structure in $\mathrm{Si}_{1-\mathrm{x}} \mathrm{Ge}_{\mathrm{x}}$ alloys......................................... 30

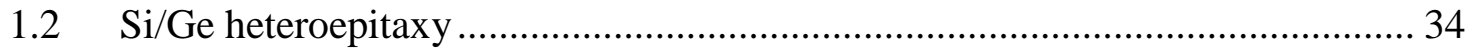

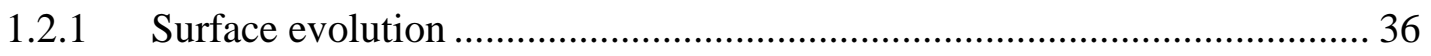

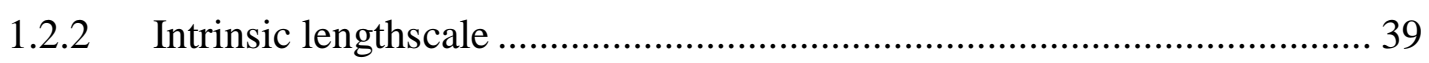

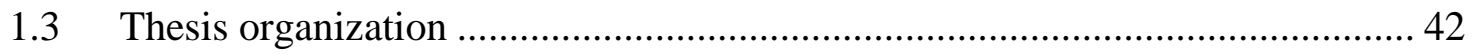

Chapter 2: Experimental and Characterization Methods ........................................ 43

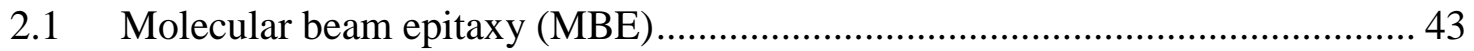

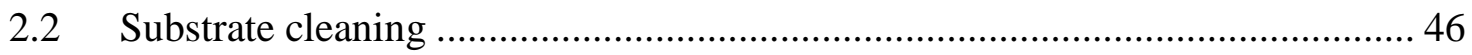

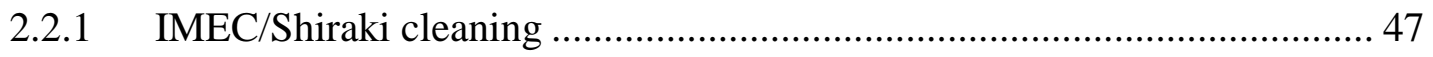

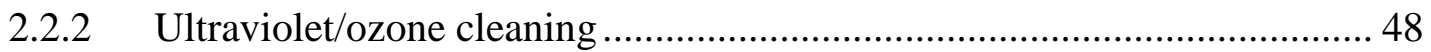

2.3 Reflection high-energy electron diffraction (RHEED) ...................................... 49

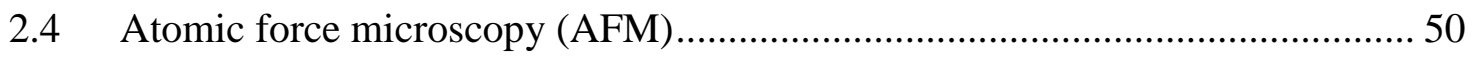

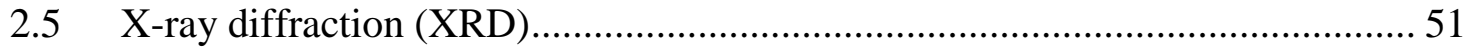

2.5.1 Lattice parameter, composition and strain characterization ....................... 54

2.5.2 Thickness measurement .......................................................................... 57

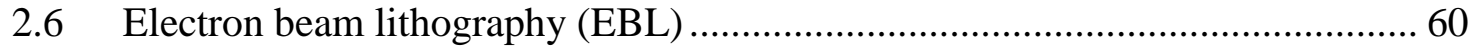

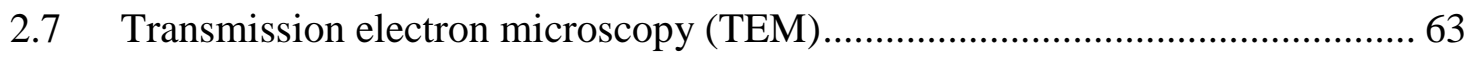

Chapter 3: Mono-layer scale chemically ordered $\mathrm{Si}_{1-\mathbf{x}} \mathbf{G e}_{\mathbf{x}}$ alloy ................................6 66

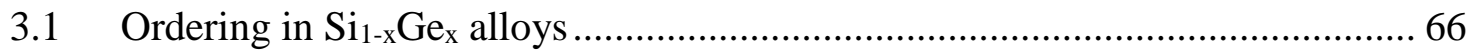

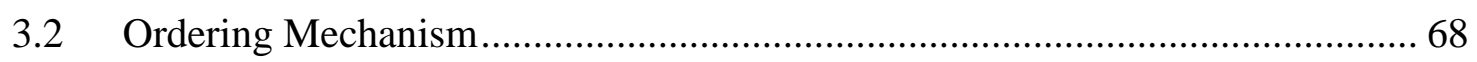




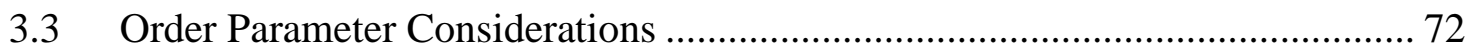

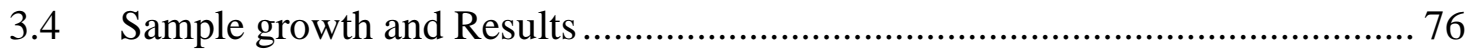

3.4.1 Critical growth temperature .................................................................. 77

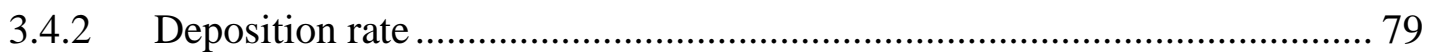

3.4.3 Domain orientation, size and symmetry ……………………................ 80

3.4.4 Epitaxial strain and growth surface morphology ....................................... 85

3.4.5 Comparative MBE grown samples ............................................................ 87

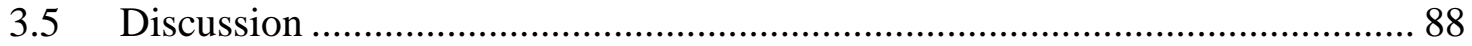

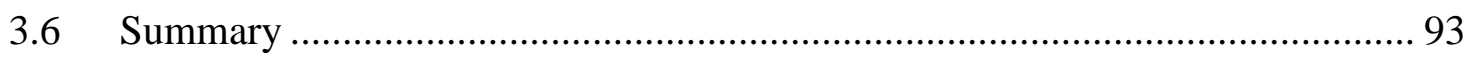

Chapter 4: Directed self-assembly of $\mathrm{Si}_{1-\mathrm{x}} \mathrm{Ge}_{\mathrm{x}}$ QDs................................................ 95

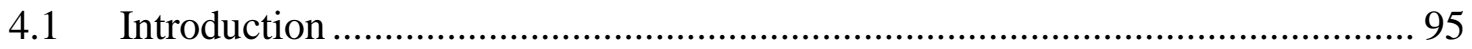

4.2 Surface chemical potential: 2D template …………….................................. 99

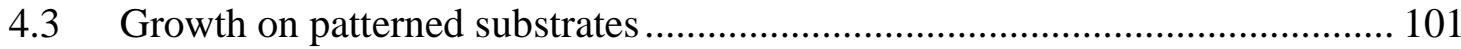

Chapter 5: Directed self-assembly of $\mathrm{Si}_{1-\mathrm{x}} \mathrm{Ge}_{\mathbf{x}}$ QDs: 2D template development..... 108

5.1 Preliminary work on FIB based patterning approach..................................... 108

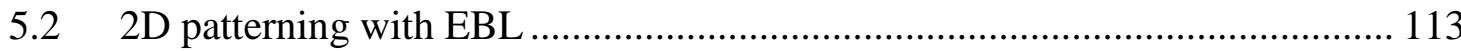

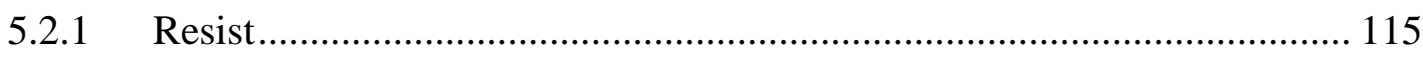

5.2.2 Exposure and EBL dose optimization.................................................. 117

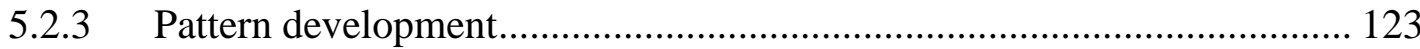

5.2.4 Pattern transfer and resist removal....................................................... 126

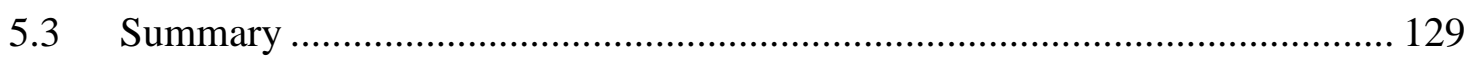

Chapter 6: Growth of $\mathrm{Si}_{1-\mathrm{x}} \mathrm{Ge}_{\mathrm{x}}$ QDs on a patterned $\mathrm{Si}(\mathbf{0 0 1})$ substrate .................... 130

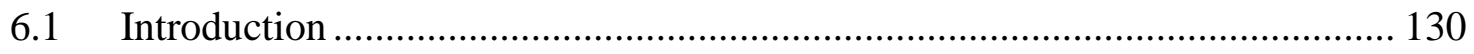

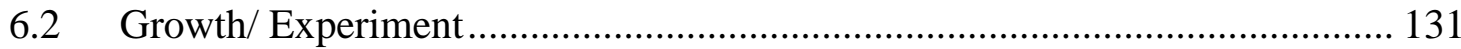

6.3 Si buffer growth: Homoepitaxy ................................................................... 132

6.4 Pattern morphology: pit-in-terrace pattern vs quasi-sinusoidal ....................... 135

6.5 Annealing time and growth temperature: Growth kinetics ............................. 136

6.6 Effects of varying $\mathrm{Si}_{0.5} \mathrm{Ge}_{0.5}$ alloy thickness .................................................. 148

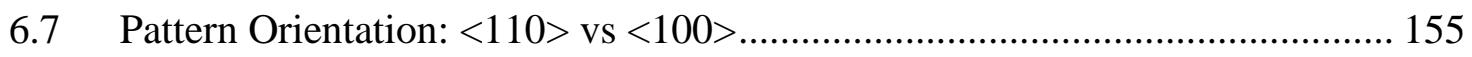

6.8 Estimation of surface diffusion length .......................................................... 158 
6.9 Site-selection of $\mathrm{Si}_{0.5} \mathrm{Ge}_{0.5}$ QDs on patterned region................................. 162

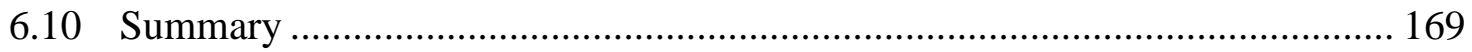

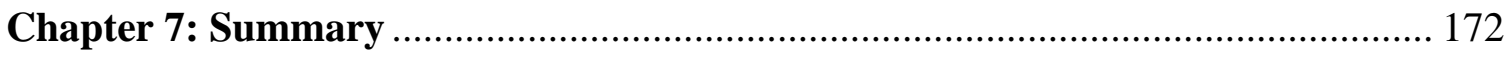

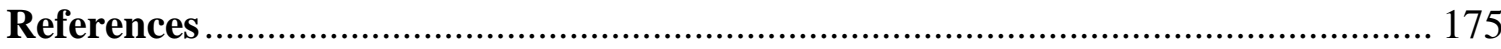

Appendix 1: Deposition rates and substrate temperature calibration................... 185

A1.1 Deposition rate calibration for DC magnetron sputter guns ......................... 185

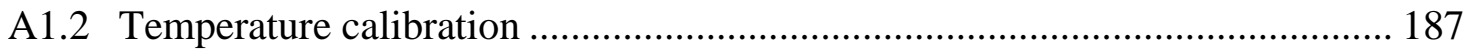

Appendix 2: IMEC-Shiraki Si cleaning process (Floro Group) ........................... 189

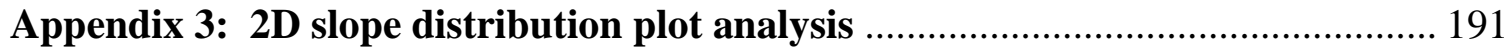

Appendix 4: Normality test: Probability plot................................................. 194 


\section{Chapter 1: Introduction}

This dissertation presents our extensive work on the structural ordering in SiGe system from nano-to-meso scale where our core motivation is driven by the enhancement in thermoelectric figure of merit (zT) achieved by structural ordering. Even though the measurement of transport properties and overall evaluation of zT does not fall under the scope of this dissertation, it serves as a foundation for our motivation for the synthesis of these structurally-ordered materials. In this chapter, we present the background and motivation for the entire dissertation. Further, we will also discuss in brief the Si-Ge heteroepitaxy which serves as a backbone for the introduction of our material system.

\subsection{Background and Motivation}

The ability to tailor material properties via synthetic physical and chemical structuring at the nano-to-meso scale, according to the fundamental paradigms of materials science, provides new opportunities to improve existing electronic device performance. This dissertation will focus on describing the strategies and efforts to investigate structural ordering of semiconducting material systems from nano-to-meso scale in order to modify electronic and thermal transport properties. The improvement of electrical transport, in particular the carrier mobility, would be important to many different device technologies. Control of thermal transport is similarly important to reducing thermal heating in scaled devices, or to improving performance in thermoelectric applications.

Thermoelectric efficiency of a material system is given by a dimensionless figure of merit (zT), 


$$
\mathrm{zT}=\frac{\mathrm{S}^{2} \sigma \mathrm{T}}{\kappa}=\frac{\mathrm{S}^{2} \sigma \mathrm{T}}{\kappa_{\mathrm{l}}+\kappa_{\mathrm{e}}}=\frac{\mathrm{S}^{2} \sigma \mathrm{T}}{\kappa_{\mathrm{l}}+\mathrm{L} \sigma \mathrm{T}}
$$

where $S$ is the Seebeck coefficient, $\sigma$ is the electrical conductivity, $\kappa$ is the thermal conductivity, here resolved into its lattice (phonon) and electronic components, and L is the Lorentz factor, making use of the Wiedemann-Franz relationship. Maximizing zT involves a delicate balance of effects that depend on the electronic and phononic dispersion, carrier concentration and effective mass, as well as scattering mechanisms for both electrical carriers and phonons. For improving zT, researchers have been focusing on the appealing concept of the "electron crystal/phonon glass", in which the material simultaneously possesses the electronic conduction properties of a single crystal, but scatters phonons like an amorphous solid. ${ }^{1}$ The basic methodology to improve thermoelectric performance is to minimize the lattice thermal conductivity while maximizing the electrical conductivity. The most commonly utilized approach for accomplishing this in commercial materials has simply been alloying of two or more chemical components. This increases phonon scattering at multiple length scales, thereby reducing " $\kappa$ ". Although electrical conductivity is adversely impacted by alloy scattering, there is nonetheless an overall increase in zT due to alloying. A review by Snyder et al., succinctly summarizes the broad strategies underlying modern research approaches aimed at improving the zT of a material. ${ }^{2}$ He identifies these as: (i) exploiting disorder within the unit cell to enhance phonon scattering while limiting reductions in electrical conduction; (ii) exploiting complex crystal structures that "separate" the phonon glass and electron crystal aspects; and (iii) incorporation of a high density of interfaces to scatter phonons 
through nanostructuring, relying on the different mean free paths for phonons vs. electrons to preserve the electrical conduction properties as much as possible.

The following section outlines a foundation for our motivation which is sub-divided into two major parts. Part I - presents the effect of nano-scale ordering on transport properties for monolayer scale chemically ordered $\mathrm{Si}_{1-\mathrm{x}} \mathrm{Ge}_{\mathrm{x}}$ thin film alloys. Part II - discusses the effect of meso-scale ordering on transport properties for quantum-dot mesocrystals (QDMC).

\subsubsection{Nano-scale ordered structure in $\mathrm{Si}_{1-\mathrm{x}} \mathrm{Ge}_{\mathrm{x}}$ alloys}

Chemically-ordered alloy systems have a unique crystal structure with their own symmetry, unit cell and associated properties that are different than their disordered counterparts. The concept of using monolayer-scale chemical ordering to tune thermal and electronic transport properties in semiconductors could offer major improvements in applications such as solid-state thermal rectification ${ }^{3}$, thermal storage devices ${ }^{4}$, transistors based on thermal switching ${ }^{5}$, thermal barrier coatings ${ }^{6}$, nanoelectronic cooling ${ }^{7}$ and thermoelectrics. ${ }^{8}$

Si-Ge alloys, which have already seen significant application in high-temperature thermoelectrics for space applications ${ }^{9}$, are known to undergo metastable chemical ordering during heteroepitaxial growth on $\mathrm{Si}(001)$ substrates. ${ }^{10-15}$ The ordering most commonly observed in $\mathrm{Si}_{1-\mathrm{x}} \mathrm{Ge}_{\mathrm{x}}$ alloys is an $\mathrm{L} 1_{1}$-like (in the Strukterbericht notation) structure, with alternating $\{111\}$ bilayers enriched in one or the other species, see Figure 1-1(a). The ordering doubles the lattice constant along the <111> direction, thereby increasing the rhombohedral primitive unit cell volume by a factor of 2 . This results in the reduction of Brillouin Zone (BZ) volume by a factor of 2. This reduced BZ size associated 


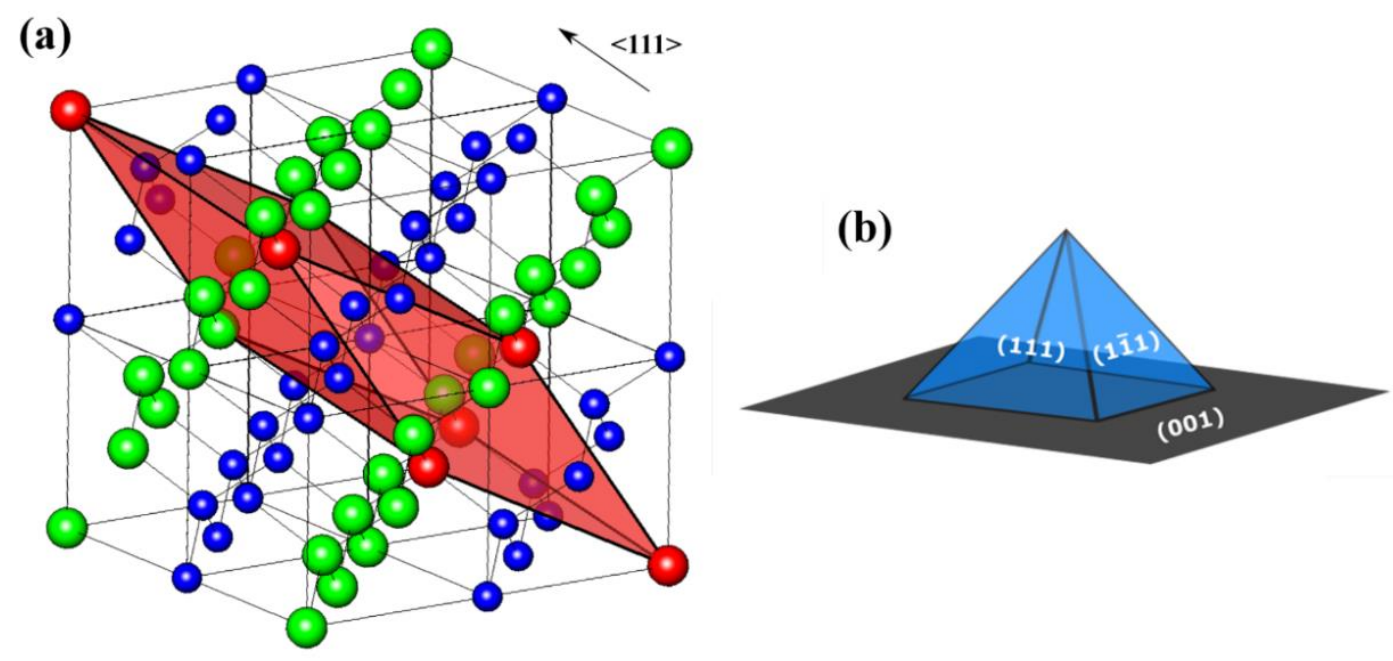

Figure 1-1: (a) $\mathrm{L}_{1}$-like ordered $\mathrm{Si}_{0.5} \mathrm{Ge}_{0.5}$ (RS2) structure, where green and blue atoms represent each chemical species. The view is along the $<321>$ direction, and shows a conventional unit cell of the ordered structure with 64 atoms. The volume shaded red color represents the primitive unit cell. (b) The four equivalent $\{111\}$ planes relative to the (001) surface that dictate the possible spatial variants for RS2 ordering.

with ordering inherently leads to enhanced Umklapp scattering of phonons, resulting in reduced lattice thermal conductivity at elevated temperature. Non-equilibrium molecular dynamics (NEMD) simulations study on the role of atomic ordering on thermal conductivity have shown that a fully ordered structures like $\mathrm{L}_{1}$ alloy can have a thermal conductivity almost as low as that of a random alloy at elevated temperature due to enhanced Umklapp scattering of phonons. ${ }^{16,17}$ Schematics of three of the L1 ${ }_{1}$-like computational cells having varying order parameter used in the NEMD study are shown in Figure 1-2. The figure also shows results for the change in thermal conductivity with temperature and order parameter.

In Figure 1-2, the degree of order, which characterizes the extent to which chemical segregation to non-random sites occur is characterized by a dimensionless order parameter, $\eta$, that ranges from $0-1$, corresponding to random-complete order respectively. The order 
parameter can be determined by diffraction-based methods. The random alloy, $\eta=0$, has only a very weak temperature dependence to $\kappa$, while the fully ordered alloy exhibits a $7 \mathrm{x}$ decrease in $\kappa$ over the range of temperature. The key result is that even a fully ordered $\mathrm{L}_{1}$ like alloy can have a thermal conductivity almost as low as that of a random alloy at elevated temperature.
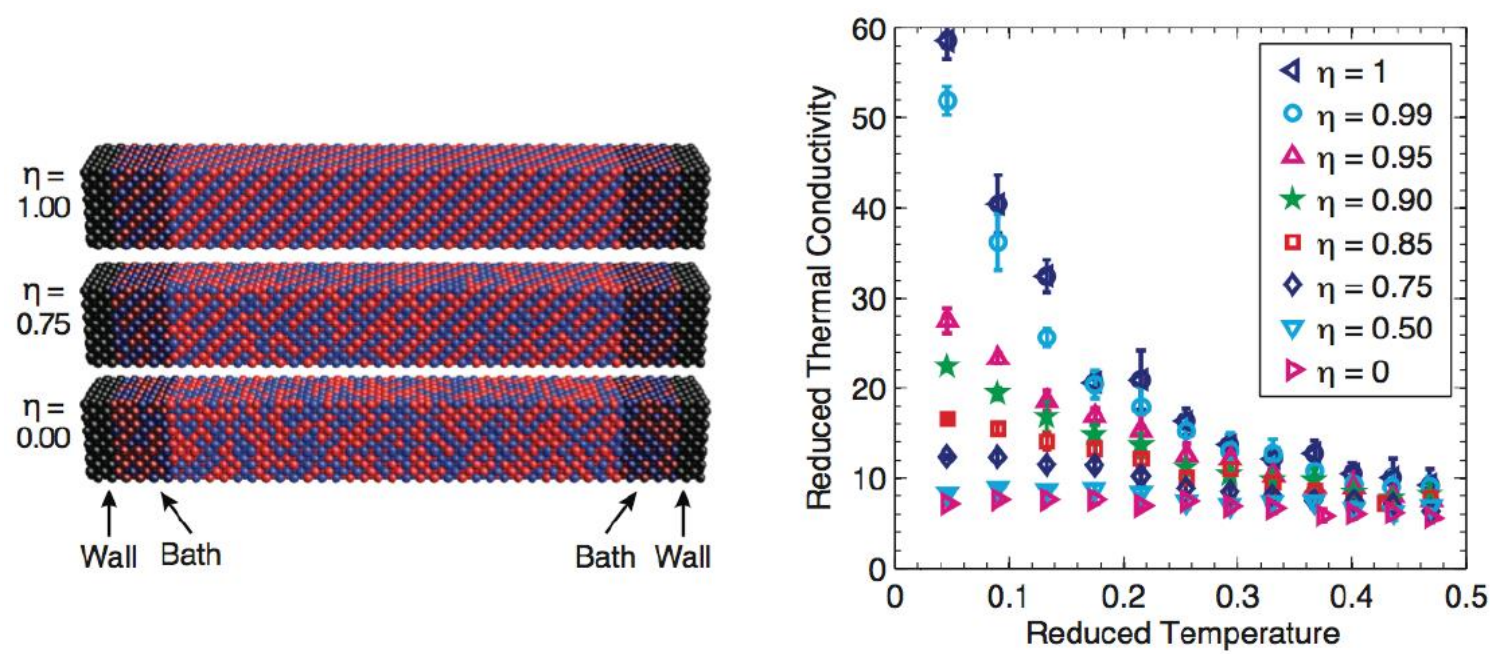

Figure 1-2: (left) Actual computational cells used in the NEMD simulations. (right) Thermal conductivity vs. $\mathrm{LJ}$ reduced temperature for order parameters from random( $\mathrm{\eta}=0)$ to fully ordered $(\eta=1) \cdot{ }^{17}$

Further, based on the behavior in ordered metallic alloys ${ }^{18}$, an ordered semiconductor alloy is expected to have an electrical conductivity significantly superior to that of a random alloy. A classic example is the $\mathrm{Cu}-\mathrm{Au}$ binary system. For system compositions near equimolar, $\mathrm{Cu}$-Au forms a disordered $\mathrm{FCC}$ solid solution at temperatures above $390{ }^{\circ} \mathrm{C}$, see Figure 1-3. Here "disordered" in this context refers to a fully crystalline structure in which chemical occupation of any FCC site is random, weighted only by the system stoichiometry. Below $390{ }^{\circ} \mathrm{C}, \mathrm{Au}-\mathrm{Cu}$ undergoes a sequence of chemical ordering transitions, and forms the so-called $\mathrm{L}_{0}$ ordered structure over an extended range of composition and temperature. The $\mathrm{L} 1_{0}$ and $\mathrm{L} 1_{2}$ ordered structures are shown in Figure 1- 
3 , and essentially represent layered structures with a repeat period of 2 monolayers. In binary systems such as $\mathrm{Cu}-\mathrm{Au}, \mathrm{Fe}-\mathrm{Pt}, \mathrm{Co}-\mathrm{Pt}$, etc., these ordered structures are thermodynamic equilibrium phases. An important observation is that the resistivity drops dramatically for chemically ordered alloys, metallic solids, due to the reduction in alloy scattering of electrons. An example is shown in Figure 1-3, where there is up to a 9x decrease in resistivity observed upon ordering.
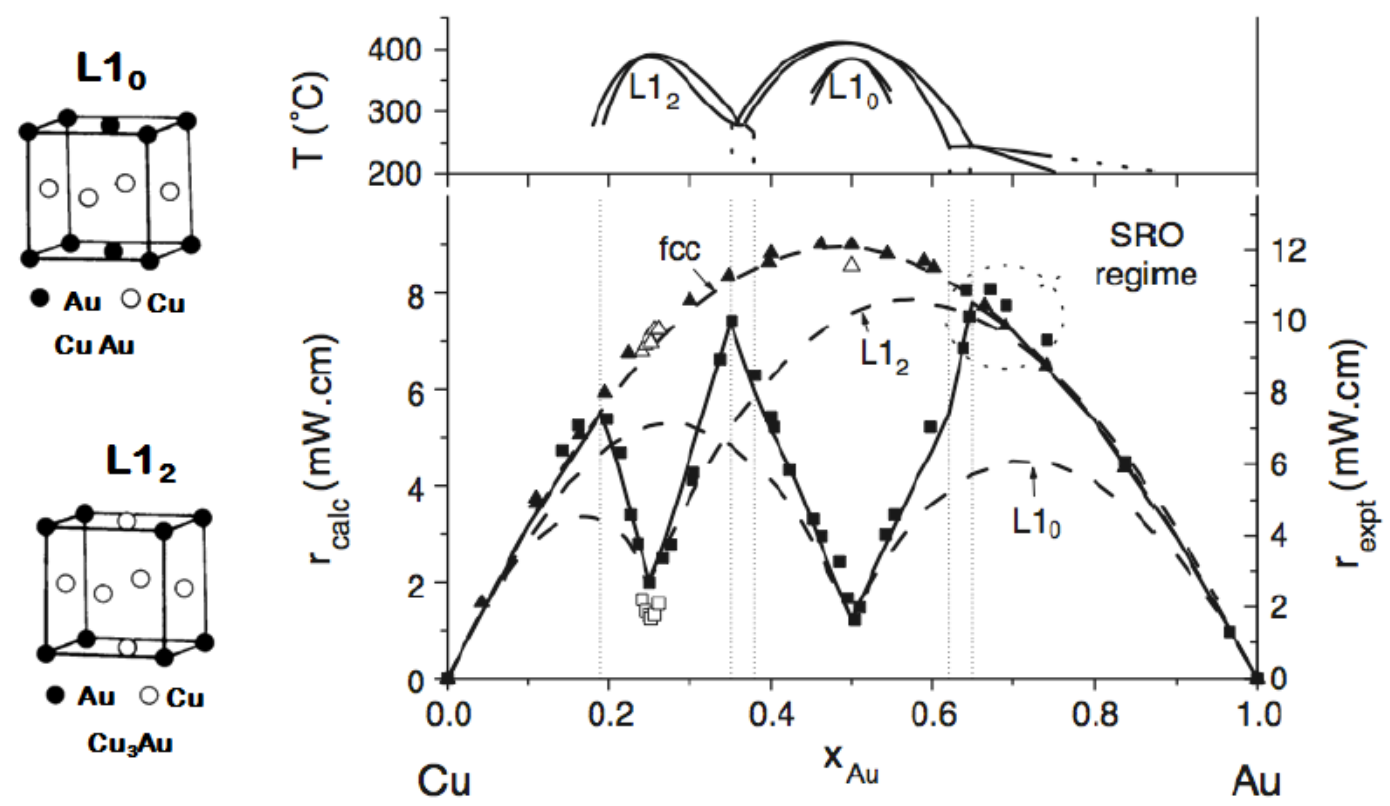

Figure 1-3: (left) $\mathrm{L}_{1}$ and $\mathrm{L}_{2}$ ordered structure in $\mathrm{Cu}-\mathrm{Au}$ system. (right) $\mathrm{Cu}-\mathrm{Au}$ phase diagram showing ordered regions along with electrical resistivity of ordered alloys from experiment (symbols) and theory (lines). ${ }^{18}$

Here, our strategy of improving thermoelectric performance best aligns with strategy (ii) as identified by Snyder et al. ${ }^{2}$ For example, implement chemical ordering to improve electrical transport characteristics like single crystals, while retain reduced thermal conductivity due to enhanced Umklapp scattering in the reduced BZ at elevated temperature. Such tuning of thermal and electronic transport properties could provide for 
increased thermoelectric performance at high temperatures where power generation from waste heat is obtained.

\subsubsection{Meso-scale ordered structure in $\mathrm{Si}_{1-\mathrm{x}} \mathrm{Ge}_{\mathrm{x}}$ alloys}

A quantum-dot mesocrystal (QDMC) is a highly ordered 3D array of quantum dots (QD) epitaxially embedded in a matrix material, also referred to as an "artificial crystal", where the interdot spacing and the size of the dots controls the mesocrystal properties. A schematic illustration of a unit cell of a QDMC is shown in Figure 1-4(a), where each QD can be considered as an "artificial atom". For the SiGe material system, $\mathrm{Si}_{1-\mathrm{x}} \mathrm{Ge}_{\mathrm{x}} \mathrm{QDs}$ embedded in a Si matrix form a Type-II band alignment, where holes are confined within the QDs and electrons are confined in the shallow potential well in-between the dots in strained Si matrix, see Figure 1-4(b). ${ }^{19}$ Such electronic structures can be ultimately used to generate new properties associated with the QDMC structure.

(a)

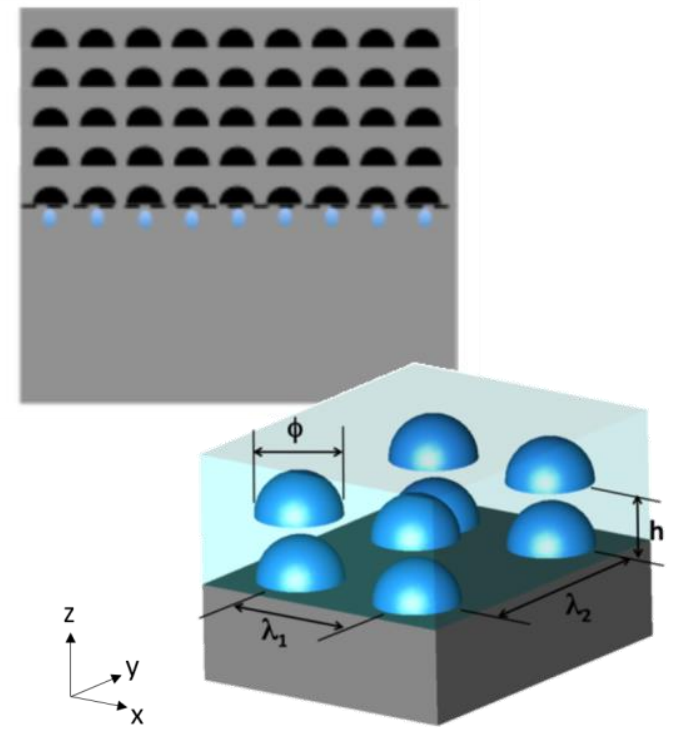

(b)

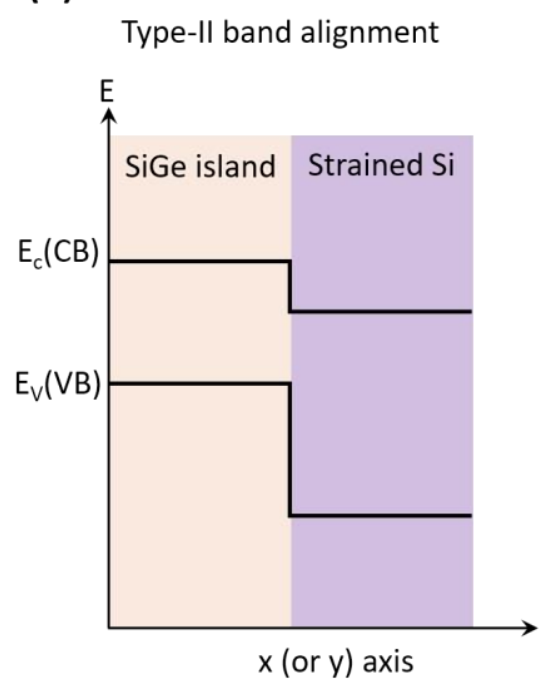

Figure 1-4: (a) Schematic illustration of QDMC (cross-sectional view) along with the unit cell, showing three lattice parameters, and the QD diameter, $\varphi$. (b) Type-II band alignment between the QDs and strained Si matrix. 
For thermoelectric applications, as described in Section 1.1, we need to tailor a material property such that it has a low lattice thermal conductivity and a large electrical conductivity. It has been shown that the thermoelectric figure of merit of such QDMC structures can be improved compared to random dot structures due to increased phonon scattering (reduced thermal conductivity, with reported thermal conductivity to be as low as $\left.1 \mathrm{WK}^{-1} \mathrm{~m}^{-1}\right){ }^{20-22} \mathrm{SiGe}$ alloys have low thermal conductivity compared to pure $\mathrm{Si}$ as alloying increases the impurity scattering of phonons. But the figure of merit zT reported in such material systems has not exceeded a value of about 1.5, even at higher temperatures, as alloying not only scatters the phonons but scattering of electrons is also increased, which adversely affects the electrical conductivity. ${ }^{2,23}$

The Si matrix embedding the QD is strained due to lattice mismatch, see Figure 1-5(a). As a result of strain, holes are confined within the QDs and electrons are localized in the strained Si on top and below, as well as around the QDs. For a highly ordered QD structures like QDMC, provided that the lateral and vertical spacing between each dot is not too large (such that the strain field overlaps with neighboring dots), a coupling of localized electron

(a)

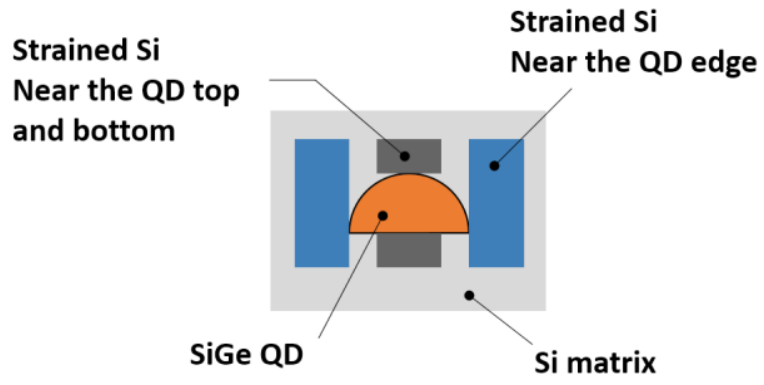

(b)

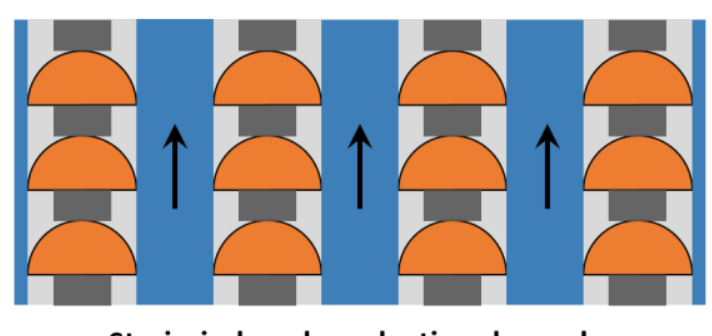

Strain-induced conductive channels

Figure 1-5: (a) A single SiGe QD embedded in a Si matrix showing the strained Si at top, bottom and edges of the QD due to lattice mismatch forming Type-II band alignment, and (c) Optimized lateral and vertical positioning of QD arrays is predicted to yield coupling of electronic states of QDs resulting in strain-induced high-mobility electron channels. 
states at the edges of QDs is predicted resulting in strain-induced high mobility electron channels as shown in Figure 1-5(b). Fromherz et al. ${ }^{24}$, from their model calculations of the 3D band structures of QDMC, indicated that extended 2D states form between each nearest-neighbor dot, due to the elastic strain imposed upon the intervening Si layer, see Figure 1-6. This is predicted to occur for a lateral interdot spacing of $35 \mathrm{~nm}$. These 2D states extend vertically throughout the mesocrystal provided that the stacked QD layers are in close proximity. Fiedler and Kratzer ${ }^{25}$ also worked out a detailed theoretical model for the electronic structure of such QDMC structures and predicted very similar 2D bands in the Si between the QDs, resulting in strain-induced conductive channels. Electron transport along the 2D bands in Si matrix was found to exhibit high mobility (>400\% relative to equivalently-doped bulk $\mathrm{Si}$ ) resulting in an enhanced zT at lower temperatures. The results of their calculation are shown in Figure 1-7 as function of temperature for conductivity, power factor and zT. Even though the model used for their computational supercell had 23 $\mathrm{nm}$ interdot spacing, the effect predicted is not directly related to center-center distance of the dots but rather edge-edge distance, which controls the ability to overlap the band states laterally. Thus, in principle, small center-to-center spacings are not required to get mobility

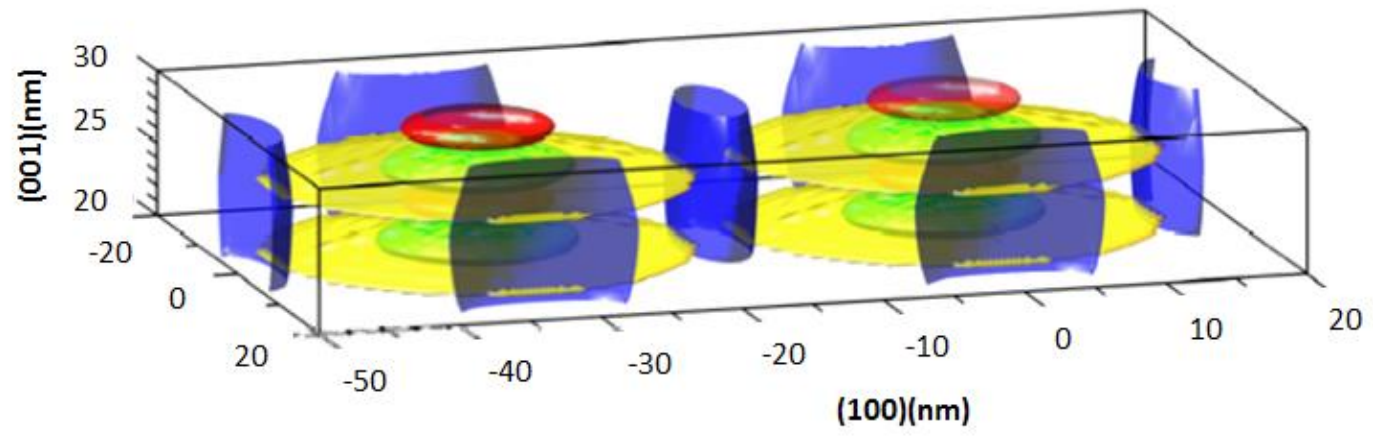

Figure 1-6: The confined electron states (blue, red, green) associated with strain imposed on the surrounding Si matrix by the Ge QDs (yellow). The blue states are of particular interest here as high-mobility conduction channels for electrons. 
enhancement. However, a smaller center-to-center distance will increase the density of the 2D conduction channels, i.e., the number of these per unit in-plane area.
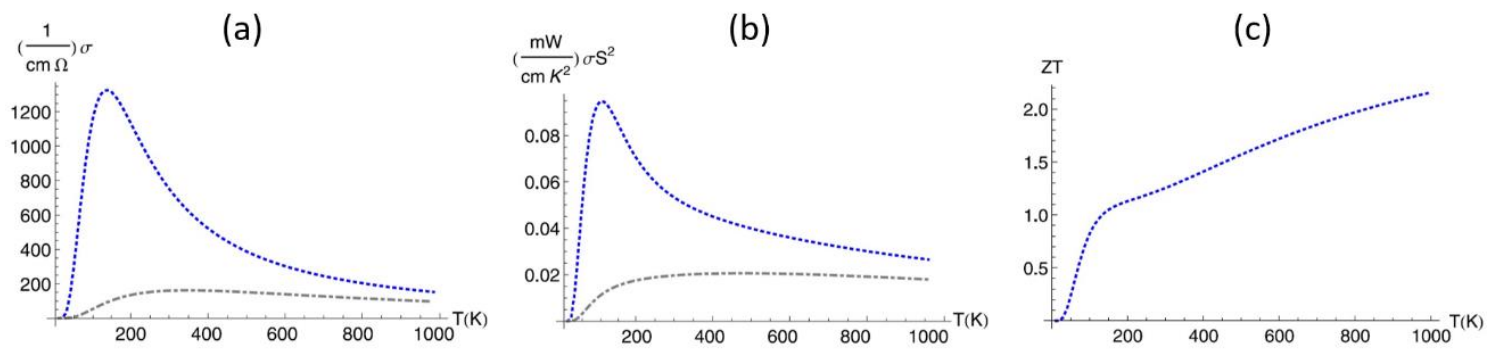

Figure 1-7: (a) Electrical conductivity, (b) power factor and (c) figure of merit - zT; for QDMC structure with doping concentrations of $10^{19} \mathrm{~cm}^{-3}$. For comparison the calculated values of bulk $\mathrm{Si}$ are shown for the same doping concentration (dashed gray).

SiGe QDs, above a critical thickness, self-assemble on $\mathrm{Si}(001)$ substrate by the development of $\{105\}$-faceted, quasi-pyramidal islands that form to relieve elastic and surface energy. ${ }^{26,27}$ The size of these QD structures are composition dependent and significant and useful modifications of transport properties have been predicted for reduced edge-edge distance between the QDs. Reducing the lateral spacing of QDs require a thorough understanding of material synthesis science including but not limited to the knowledge of fundamental origin of QD formation and coarsening suppression mechanisms. 


\subsection{Si/Ge heteroepitaxy}

Silicon and Germanium are two of the most commonly used materials for semiconductor device fabrication. In addition to the large expertise base of industrial processing for these group IV elements, non-toxicity, ease of doping and its ability to form high quality crystals have made $\mathrm{Si}$ and $\mathrm{Ge}$ a leading material for semiconductor device applications. Both $\mathrm{Si}$ and Ge have a diamond cubic crystal structure with lattice constants of asi $=5.4309 \AA$ and $\mathrm{a}_{\mathrm{Ge}}=5.6575 \AA$ at $300 \mathrm{~K}$. They exhibit a complete solid solubility in diamond phase, see Figure 1-8.

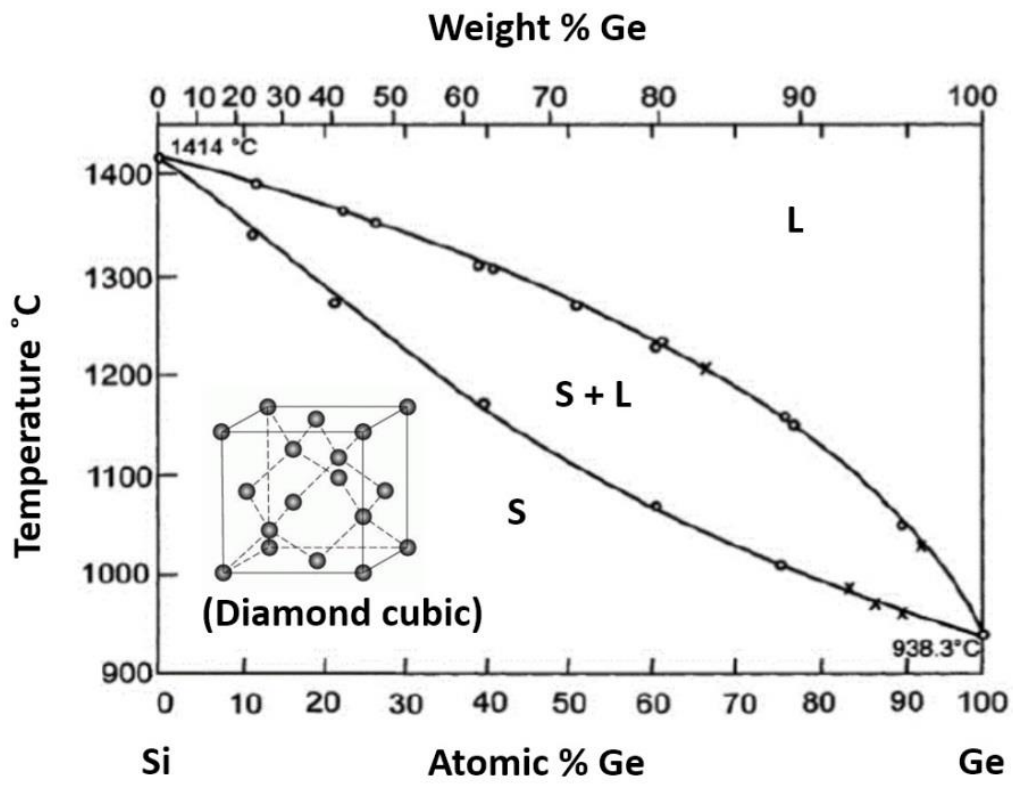

Figure 1-8: Binary phase diagram of Si-Ge

For $\mathrm{Ge}$ and $\mathrm{Si}_{1-\mathrm{x}} \mathrm{Ge}_{\mathrm{x}}$ films deposited onto a $\mathrm{Si}$ substrate, a biaxial misfit strain parallel to the substrate interface arises for a fully coherent film, given by,

$$
\varepsilon_{\text {coh }}=\frac{a_{\text {subst }}-a_{\text {film }}}{a_{\text {film }}},
$$




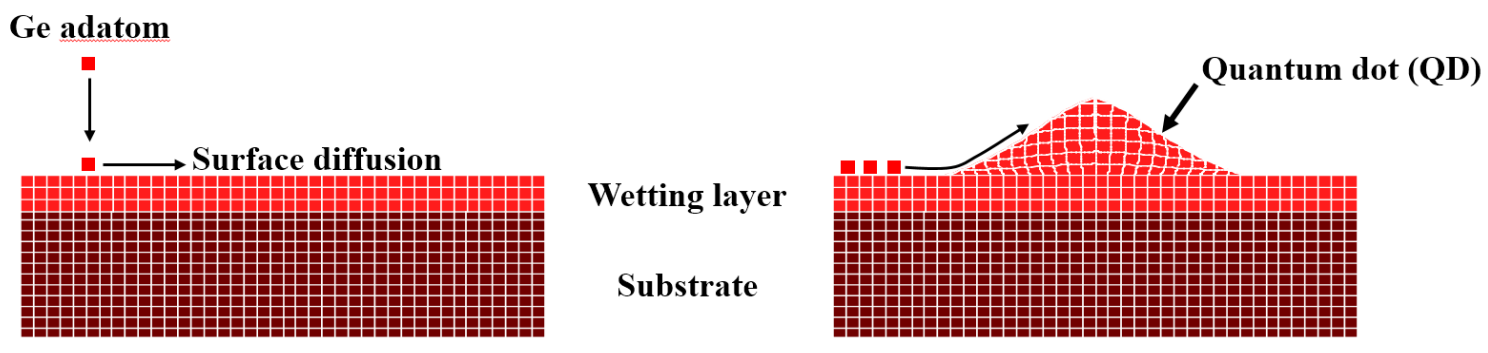

Figure 1-9: Schematic representation of the S-K growth mode

For pure Ge deposited on a single crystal Si substrate, the $4.2 \%$ lattice mismatch results in the evolution of Ge QDs to partially relax the strain via Stranski-Krastanov (S-K) growth mode. ${ }^{28}$ In the S-K growth mode of Ge/Si (001), initially a planar Ge wetting layer (WL) grows pseudomorphically matching the lattice constant of the Si substrate. After reaching a critical WL thickness of 3-4 $\mathrm{ML}^{29}$, Ge layer relaxes by forming faceted 3D islands, in order to relax some of the stored elastic energy, see Figure 1-9. Ge QDs initially selfassemble on Si substrate by the development of $\{105\}$-faceted coherent quasi-pyramidal islands. With further Ge deposition the pyramidal islands transition to domes and superdomes, where each shape has increasingly steeper $\{h \mathrm{hl}\}$ facets, see Figure $1-10 .^{26,27,30}$ Thus, QDs are 3D structures that form to relieve the strain induced due to lattice mismatch during heteroepitaxy and strain relaxation is the common driving force that results in the

(a)

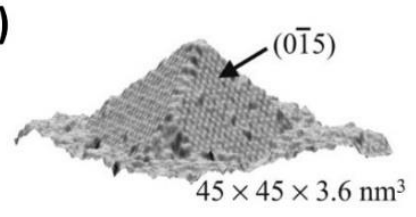

(b)

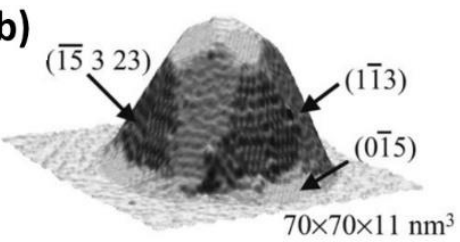

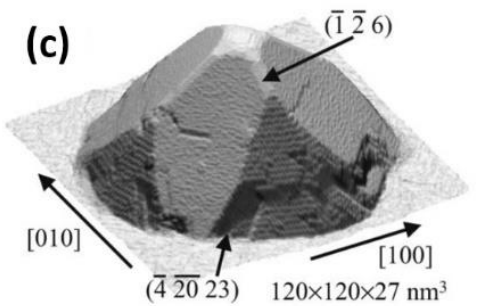

Figure 1-10: STM images of Ge islands on $\mathrm{Si}(001)$ : (a) pyramid, (b) dome and (c) superdome. ${ }^{30}$ 
evolution of a flat and strained 2D surface to a faceted 3D surface. However, the simplified S-K growth mode does not describe the early stages of 2D/3D transition characterized by surface roughening or morphological instability.

\subsubsection{Surface evolution}

For the growth of $\mathrm{Si}_{1-\mathrm{x}} \mathrm{Ge}_{\mathrm{x}}$ on $\mathrm{Si}(001)$ substrate, the first stage is the layer-by-layer growth resulting in the formation of flat and strained 2D wetting-layer (WL). Strain relaxation is the common driving force which results in the surface morphological evolution in these epitaxial films. The overall energy of the strained film in such case is simply a sum of elastic energy and the surface energy. Compared to bulk, the surface energy cannot be neglected in thin films. Therefore, initially the film is essentially flat and smooth in order to limit the surface energy.

As the growth progresses, the compressive strain in the film is relaxed by the formation of dimer vacancies, kinks, Ge segregation and increased surface step density resulting in a gradual rise of surface roughness. The initial stage of roughening is characterized by a nucleationless morphological instability regime where a periodic corrugation is induced, see Figure 1-11. The surface roughness at this stage can exhibit a characteristic lateral lengthscale termed as intrinsic lengthscale $\left(\lambda_{\mathrm{ATGS}}\right)$ or Asaro-Tiller-Grinfeld-Srolovitz

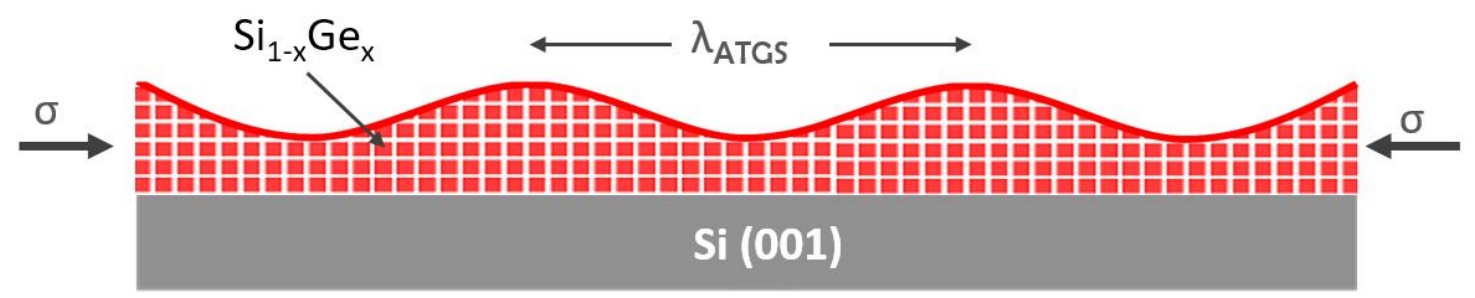

Figure 1-11: Schematic illustration showing the development of instability during the early stages of $\mathrm{Si}_{1-\mathrm{x}} \mathrm{Ge}_{\mathrm{x}}$ alloy growth. 
(ATGS) instability ${ }^{31-33}$. Formation of quantum dots has also been modeled in terms of classical nucleation theory, resulting in a critical nucleus size ${ }^{34,35}$. The scaling of the intrinsic wavelength (or critical size) is discussed below.

As the growth further progresses, the roughness mounds as shown in Figure 1-11, defined by morphological instability, evolves into prepyramids with rounded shapes which ultimately transforms to a $\{105\}$ faceted truncated pyramidal structure, see Figure 1-12 for the initial stages prior to a complete pyramidal formation and see Figure 1-13(a) for free energy change during the evolution.
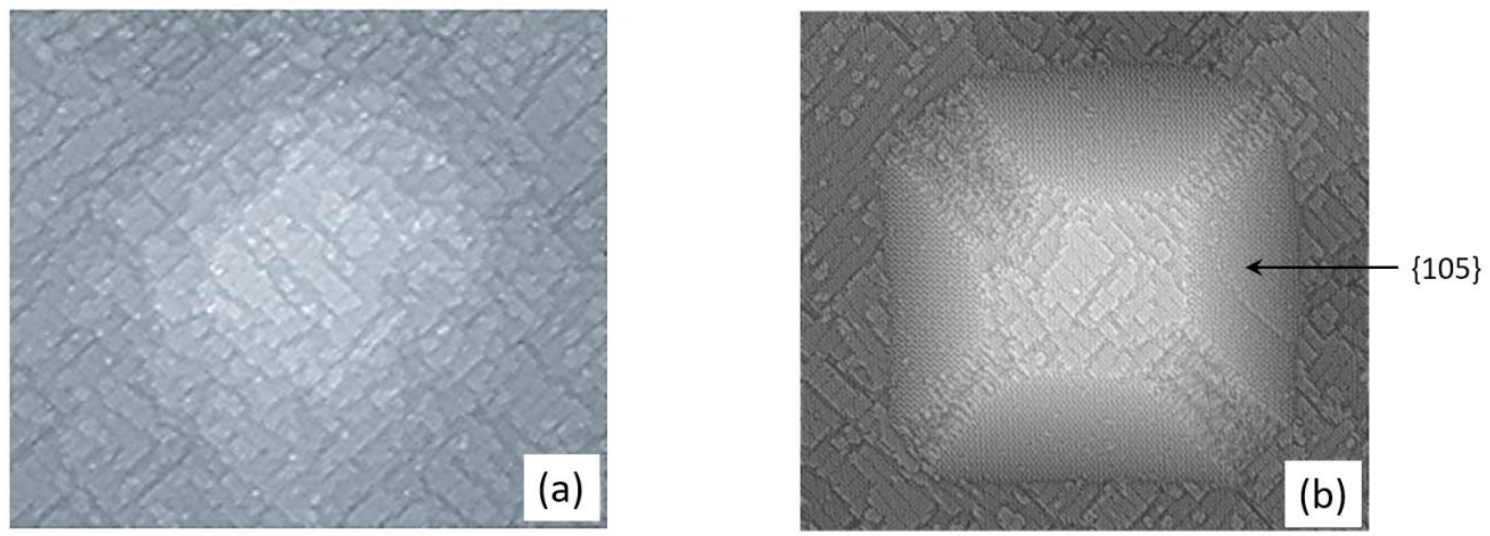

Figure 1-12: STM images of (1) prepyramid island and (b) truncated $\{105\}$ faceted pyramid prior to the formation of a complete $\{105\}$ faceted quasi-pyramidal $3 \mathrm{D}$ islands. ${ }^{37}$

Further into the growth, these $\{105\}$ faceted quasi-pyramidal structures evolve into domes and superdomes as described in the previous section. A kinetic phase diagram representing various growth regimes of $\mathrm{Si}_{1-\mathrm{x}} \mathrm{Ge}_{\mathrm{x}}$ growth as a function of the deposited thickness and strain (or composition) is shown in Figure 1-13(b). This phenomenon of morphological instability is commonly observed for $\mathrm{Si}_{1-\mathrm{x}} \mathrm{Ge}_{\mathrm{x}}$ system at low misfit strain $(\mathrm{x} \leq 0.6) \cdot{ }^{36-41}$ For system with larger misfit strain or higher Ge composition, growth instability develops 
much faster, resulting in a conventional 3D island nucleation and growth. ${ }^{40}$ For instance with reference to Figure 1-13(b), region-II and region-III is very narrow at higher strain (or higher Ge composition) resulting in a sudden 2D-3D transition with a direct formation of 3D islands.
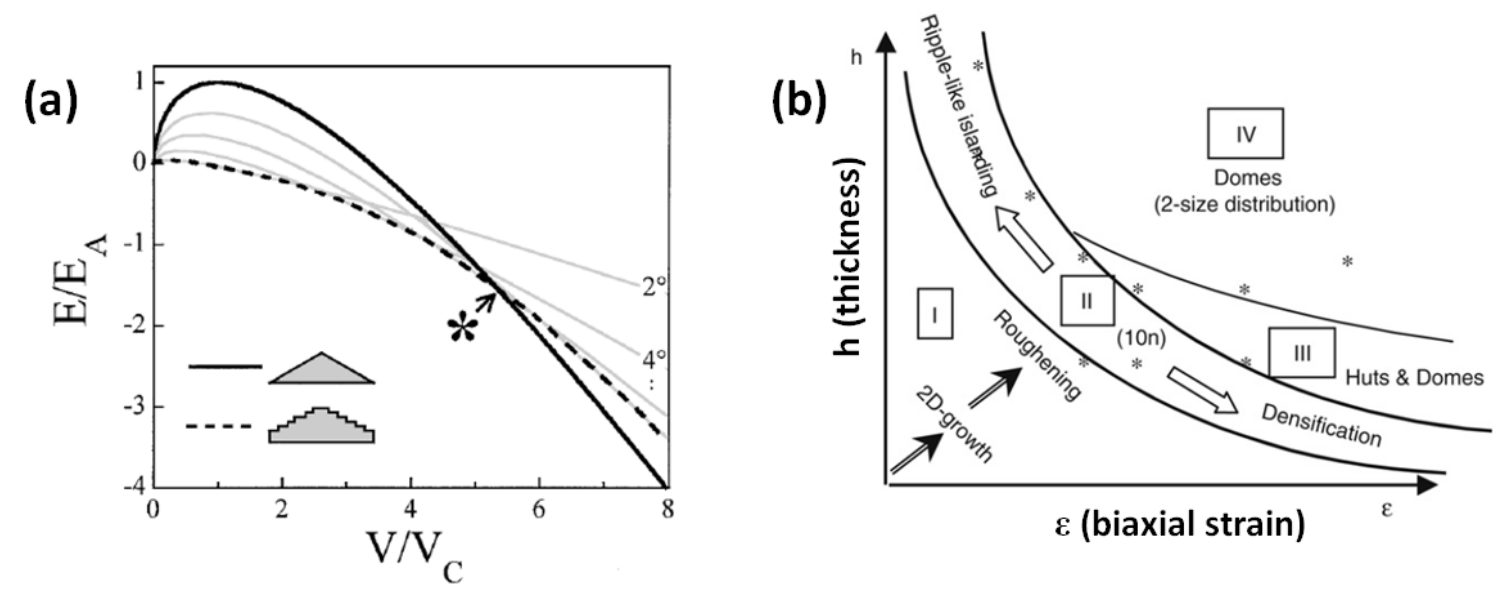

Figure 1-13: (a) Free energy E (normalized to the activation energy $E_{A}$ for nucleation of (105) faceted huts) as a function of island volume $\mathrm{V}$ (relative to critical volume of a hut $V_{c}$ ). The solid line shows the energy for (105) facet island and the dashed line shows the evolution path where the sidewall angles grow continuously from $0^{\circ}-11^{\circ} .^{36}$ (b) Kinetic phase diagram representing the main growth regimes of $\mathrm{Si}_{1-\mathrm{x}} \mathrm{Ge}_{\mathrm{x}}$ layers. ${ }^{140}$

It should be noted that the surface evolution during SiGe heteroepitaxy to reduce the strain energy is only effective at lower thickness. As the growth progresses, at larger thickness, the larger strain in the film will ultimately be relaxed by the introduction of misfit dislocations at the substrate interface. There exists a critical thickness, above which the misfit dislocations are introduced to relieve the strain. Figure 1-14 shows the critical thickness calculated by Matthews and Blakeslee ${ }^{42}$ plotted against Ge fraction for $\mathrm{Si}_{1-\mathrm{x}} \mathrm{Ge}_{\mathrm{x}}$ layers grown on $\mathrm{Si}(001)$. 


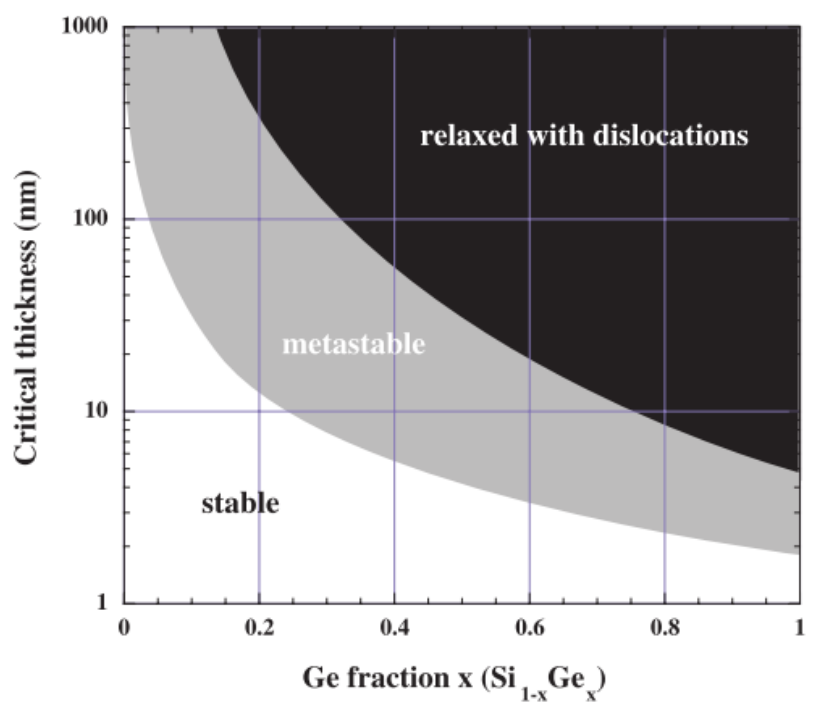

Figure 1-14: The Matthews and Blakeslee critical thickness plotted against Ge fraction $(\mathrm{x}){ }^{42,141}$

\subsubsection{Intrinsic lengthscale}

QD formation requires growth conditions where adatom diffusion lengths must match or exceed the intrinsic length scale ( $\left.\lambda_{\mathrm{ATGS}}\right) .{ }^{33}$ This "wavelength" arises during the early stages of the $2 \mathrm{D} / 3 \mathrm{D}$ transition due to the competition between the reduction of the elastic energy by islanding vs. the increase in surface area. The morphological instability in semiconductor epitaxy was first reported in InGaAs systems ${ }^{43}$ and then later in $\mathrm{Si}_{1-\mathrm{x}} \mathrm{Ge}_{\mathrm{x}}$ systems ${ }^{44-46}$. In a simple notion, the ATGS wavelength is found to have inverseproportional relation to the Young modulus and misfit square. Sutter and Lagally ${ }^{36}$ experimentally verified for SiGe systems, intrinsic length decreases with increasing $\mathrm{Ge}$ concentration and deduced the relation - $\lambda_{\text {ATGS }} \alpha \varepsilon^{-(1.0 \pm 0.1)}$. Tromp et al..$^{40}$ showed that in SiGe systems morphological evolution was evident for composition $0.2 \leq \mathrm{x} \leq 0.6$. For $\mathrm{x}>$ 0.6 evolution was observed to be similar to that of pure Ge i.e, conventional direct 3D island nucleation. The growth instability develops much faster for higher strain or higher 
Ge composition systems as discussed in previous section. The instability value was worked out in detail by Asaro and Tiller ${ }^{31}$, Grinfeld $^{32}$ and later Srolovitz ${ }^{33}$, given by

$$
\lambda_{\mathrm{ATGS}}=\frac{4 \pi}{3} * \frac{\gamma_{\mathrm{WL}}}{\mathrm{M} * \varepsilon^{2}}
$$

where $\gamma_{\mathrm{WL}}$ is the surface energy and $M \varepsilon^{2}$ is the elastic energy density in the film due to lattice mismatch. The biaxial modulus " $\mathrm{M}$ " is given by

$$
\mathrm{M}=\mathrm{C}_{11}+\mathrm{C}_{12}-\frac{2 \mathrm{C}_{12}^{2}}{\mathrm{C}_{11}}
$$

where the elastic moduli " $\mathrm{C}_{11}$ " and" $\mathrm{C}_{12}$ " for $\mathrm{Si}$ and Ge is 166.2, 64.4 and 128.4, 48.2 GPa respectively. Here, Equation 1-4 gives the biaxial modulus value for a pure element (Si or $\mathrm{Ge}$ ). Thus for an alloy, in our case $\mathrm{Si}_{1-\mathrm{x}} \mathrm{Ge}_{\mathrm{x}}$, the biaxial modulus is given by ${ }^{47}$

$$
\mathrm{M}_{\mathrm{Si}_{1-\mathrm{x}} \mathrm{Ge}_{\mathrm{x}}}=\mathrm{M}_{\mathrm{Si}}+\frac{\mathrm{a}_{\mathrm{Si}}\left(\mathrm{M}_{\mathrm{Ge}}-\mathrm{M}_{\mathrm{Si}}\right)}{\left(\mathrm{a}_{\mathrm{Si}}-\mathrm{a}_{\mathrm{Ge}}\right)} * \varepsilon_{(\mathrm{x})}
$$

See Figure 1-15(a), where $M_{\text {SiGe }}$ is the slope $\left(\sigma=M^{*} \varepsilon\right)$. For the calculation of $\lambda_{\mathrm{ATGS}}$ from Equation 1-3 the only required value now is $\gamma_{\mathrm{WL}}$. For pure Ge the (001) surface under
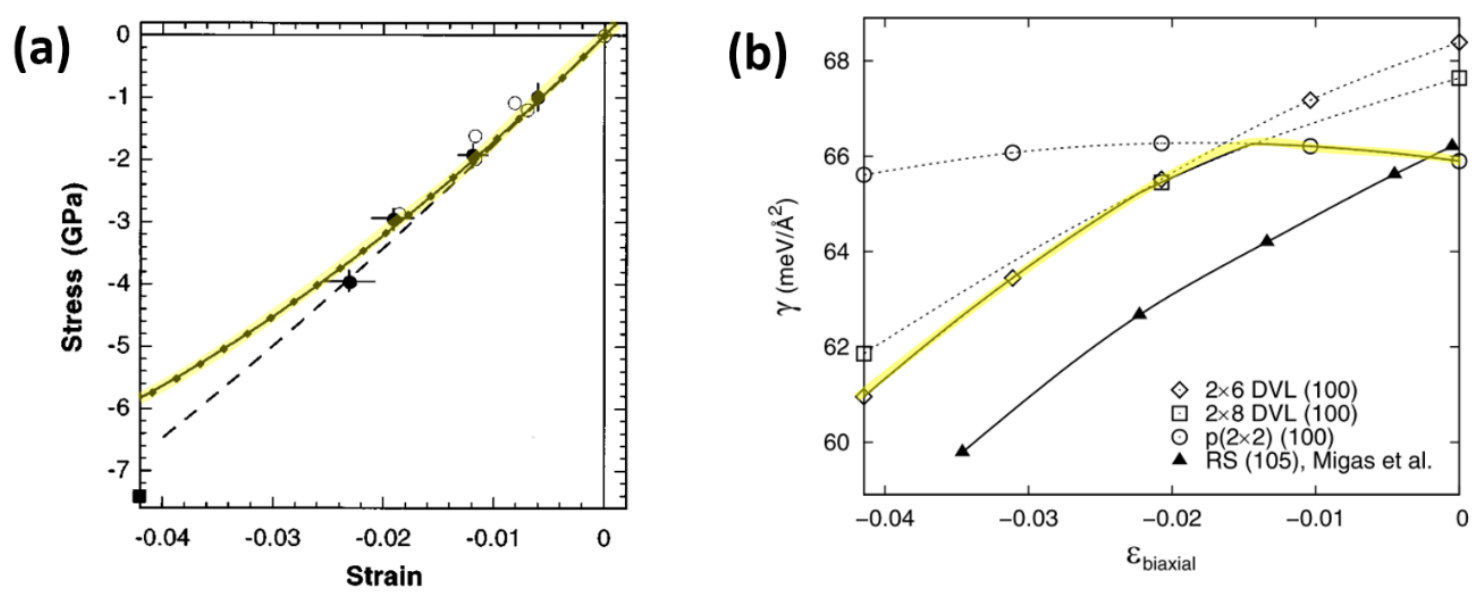

Figure 1-15: (a) Measured stress-strain data in SiGe system calculated by Floro et al. ${ }^{47}$ The solid curve (highlighted) is the quadratic fit to the data from which the $\mathrm{M}_{\mathrm{SiGe}}$ can be calculated, (b) Surface energies versus biaxial strain for Ge surface under strain. Highlighted solid line refers to the (001) Ge surface energy under various biaxial strain. ${ }^{48}$ 
compressive strain, the wetting layer surface energy is $61 \mathrm{meV} / \AA^{2}$ from first principles calculations. ${ }^{48}$ Thus, with Equation 1-3 $\lambda_{\text {ATGS }}$ value for a pure Ge is calculated to be $\sim 16 \mathrm{~nm}$. With an assumption that the surface in SiGe film is Ge rich (due to Ge segregation ${ }^{49}$ ) the $\gamma_{\mathrm{WL}}$ value for a SiGe film can then be taken as $\gamma_{\mathrm{WL}}$ value of pure Ge (001) under various strain condition, see Figure 1-15(b).

Therefore, from Equation 1-3 for $\mathrm{Si}_{0.5} \mathrm{Ge}_{0.5}$, using $\mathrm{M}=161.7 \mathrm{GPa}, \gamma=65.5 \mathrm{meV} / \AA^{2}$, and $\varepsilon$ $=-0.0195$, we obtain $\lambda_{\text {ATGS }} \approx 72 \mathrm{~nm}$. Figure $1-16$ shows the dependence of $\lambda_{\text {ATGS }}$ on Ge concentration of the alloy film, calculated from Equation 1-3. Further, we have included the reported value from literature at various compositions for comparison with our calculated data.

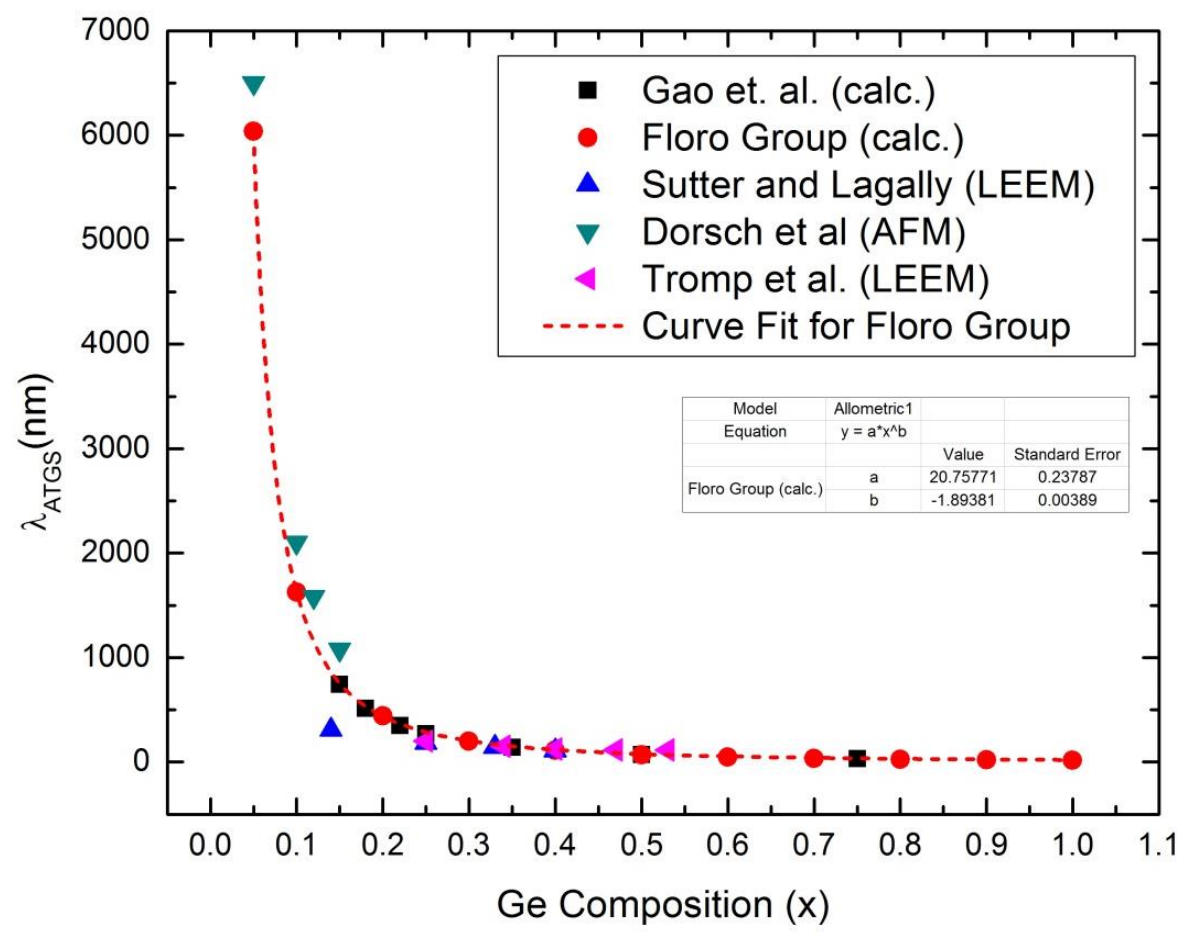

Figure 1-16: $\lambda_{\text {ATGS }}$ plotted against Ge concentration (x), showing our calculated data with experimental and theoretical data from the literature. 


\subsection{Thesis organization}

The background and motivation for the entire thesis work has already been described in this chapter along with a brief introduction for the $\mathrm{Si} / \mathrm{Ge}$ heteroepitaxy which serves as a backbone for the introduction of our material system. The thesis is organized in the following manner: in Chapter 2 the specifics of our growth and characterization methods used during our experimental works are discussed. For instance, details about the growth chamber setup, cleaning methods used for growth substrates, patterning methodology etc., have been discussed in details. In Chapter 3, results for the growth of $\mathrm{Si}_{0.5} \mathrm{Ge}_{0.5}$ alloy film is presented along with a detail discussion regarding the monolayer scale chemical ordering observed in these alloys. Chapter 4 discusses the science of directed self-assembly of QDs along with a brief review of QD growth on patterned substrates. In Chapter 5, we present the process and methodology used for creating 2D templates on Si substrates followed by results and discussion of QDs growth on such 2D templated substrates in Chapter 6. And finally, in Chapter 7 we finish by discussing the key results and future outlook of our current work. 


\section{Chapter 2: Experimental and Characterization Methods}

\subsection{Molecular beam epitaxy (MBE)}

Molecular beam epitaxy (MBE) is one of the experimental techniques that utilize ultrahigh-vacuum (UHV) environment to produce high quality epitaxial structures with a control of deposition at monolayer (ML) scale. The design of MBE and growth techniques depends on the desired structure, type of material and needs. However, the underlying principle of all MBE growth is essentially the same: atoms or cluster of atoms are created and allowed to migrate in the vapor through an UHV environment. They then impinge and

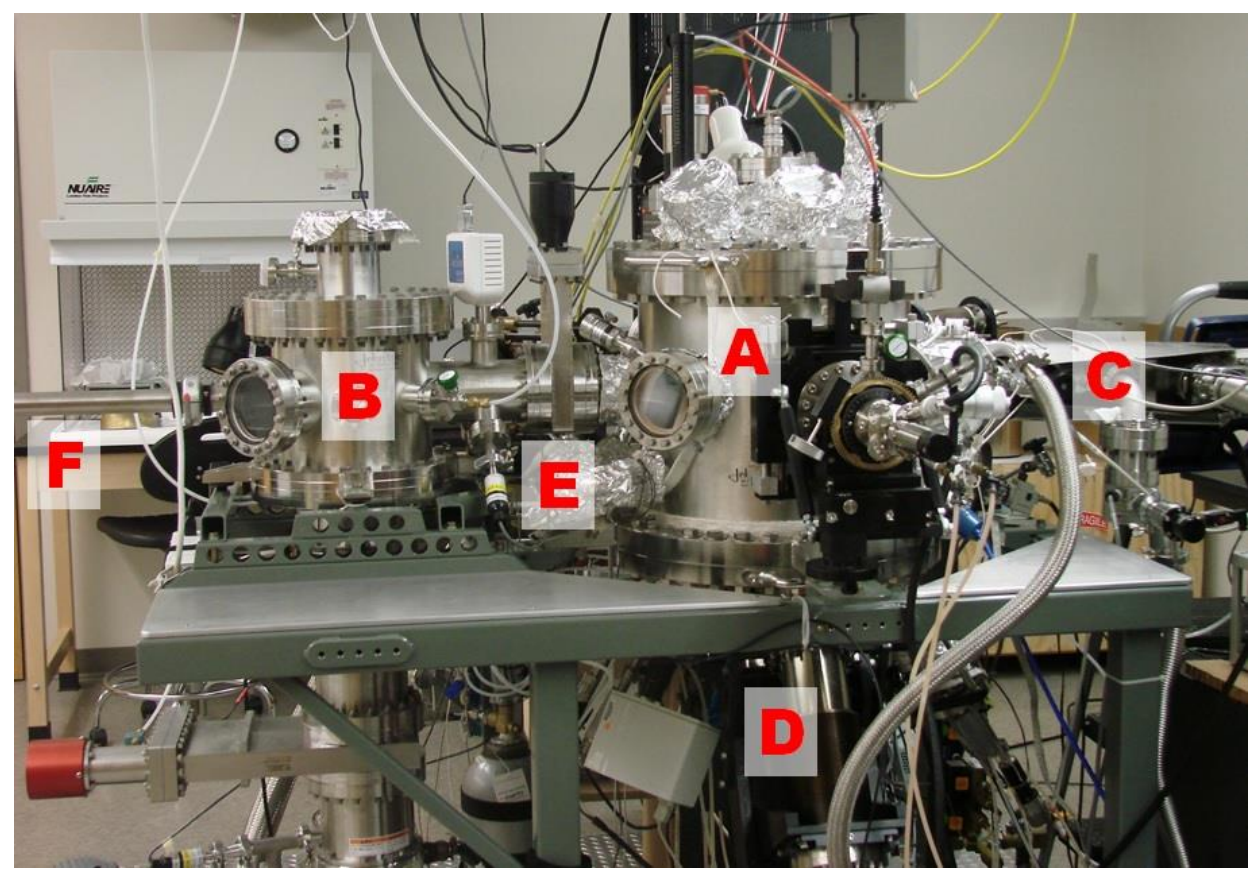

Figure 2-1: UHV Hyperthermal MBE setup at UVa showing key components for film synthesis. [A]- MBE chamber, [B]-Load Lock chamber, [C]- RHEED gun, [D]- Sputter guns, [E]- Manual gate valve, [F]- Transfer arm.

condense on a single crystal substrate, often held at elevated temperature to promote surface diffusion and for epitaxial growth. Apart from just being a high purity material deposition chamber, MBE offers highly-controlled growth of ultra-thin layers, atomically 
smooth heterointerfaces, and tailored doping profiles, enhanced by the potential for lower growth temperatures and deposition rates. ${ }^{50}$ All of the growth experiments included in this dissertation were conducted in our custom, UHV hyperthermal MBE (HyperMBE) system at UVa, see Figure 2-1. The HyperMBE is designed to access a broad window of kinetic deposition parameters.

Generally, a Group IV MBE is equipped with thermal evaporation and/or electron beam evaporation system for material deposition which is operated under UHV to minimize chances of contamination. However, these evaporation techniques do have certain disadvantages like extensive outgassing, poor rate stability and thermal loading of the sample. In contrast to these thermal deposition techniques, our HyperMBE employs variable-distance direct current (DC) magnetron sputter guns for deposition of Ge and Si. Magnetron sputtering has been shown to yield high-quality Si and Ge growth surfaces by

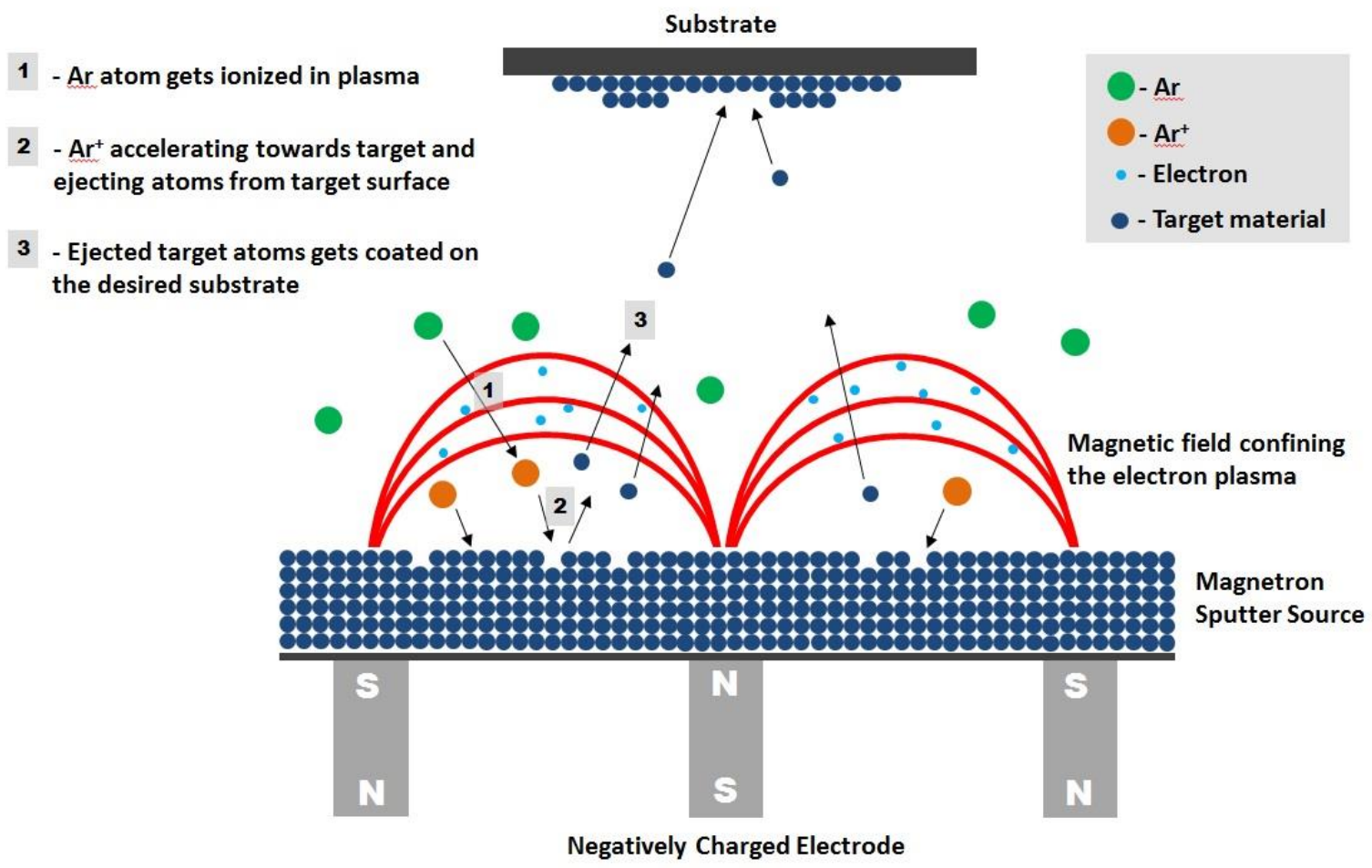

Figure 2-2: Schematic of a magnetron sputtering process. 
scanning tunneling microscopy, when performed under otherwise very clean conditions. ${ }^{51,52}$ Sputtering is a deposition technique utilizing a gaseous plasma to create ions $\left(\mathrm{Ar}^{+}\right)$which are then accelerated into some negatively charged source material (like Si and Ge target materials as cathode). Atoms get ejected from the target material as a result of ion bombardment which will then get coated to a substrate. In magnetron sputtering, strong magnets are placed behind the target material to trap the free electrons in a magnetic field directly above the target surface. The increase in the density of free electrons near the target surface, enhances the probability of ionizing a neutral Ar atom by several orders of magnitude, resulting in a higher plasma density at lower working pressures, see Figure 22. For sputtering, the energy of the bombardment for $\mathrm{Ar}^{+}$is greater than the surface binding energy $\left(E_{B}\right)$ of the source targets, in our case, $E_{B}$ of $S i$ and Ge are $4.73 \mathrm{eV} /$ atom and 4.29 eV/atom respectively. ${ }^{53}$ The energetics of ejected species during deposition plays a vital role in the crystal growth quality. The estimated energies of the ejected target species for our sputter system are in the range of 1-20 eV, which is described by the Thompson

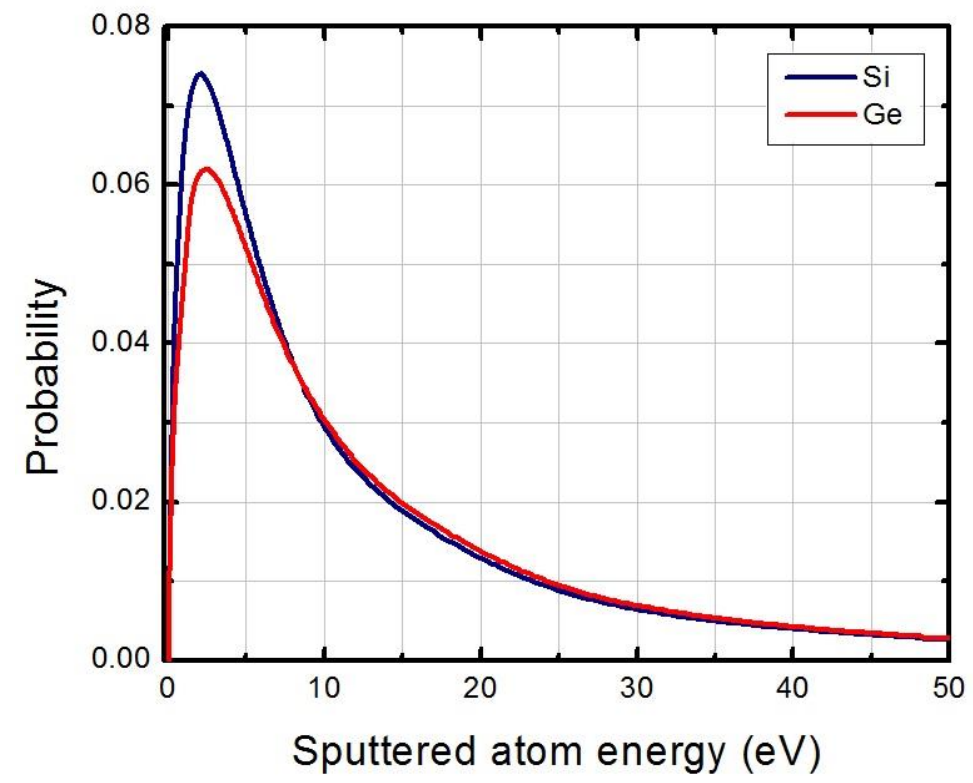

Figure 2-3: Probability distribution of sputtered atom energy from the target. ${ }^{54}$ 
distribution, see Figure $2-3 .{ }^{54}$ Of course, the sputtered atoms will lose energy to collisions with the background gas during transit from the target to the substrate. This distance can be varied in our chamber, in order to vary the degree of thermalization.

Deposition rates for our magnetron sputter guns can be varied between $0.05 \AA / s$ and 6.5 $\AA / s$ depending on power and substrate-target separation; see Appendix 1 for the calibration rates. Both $\mathrm{Si}$ and Ge sputter sources are mounted on a 6 inch linear translation stage which allows the substrate-target separation to be adjusted between 2 and $12 \mathrm{~cm}$. These sputter guns are DC biased, with up to 80 Watts incident power. The deposition rates from both the sputter guns were calibrated using a quartz crystal rate monitor (QCM). During the deposition, sputtering requires the back filling of the chamber with a non-reactive Ar gas to 5-10 mTorr pressures. To minimize the contamination, high purity Ar gas (99.9995\% pure) line is fitted with a hot getter pump, cold getter pump and a liquid nitrogen trap in a series before the introduction of gas into the chamber through a fine leak valve to minimize contamination. The getters are designed to reduce contamination to parts per billion. Sputtering has other benefits however, including higher deposition rates, a wider growth parameter space, as well as the ability to deposit high melting point materials without high thermal loading of the targets.

\subsection{Substrate cleaning}

Surface contaminants on a wafer exist as absorbed ions and elements, thin films, discrete particles, particulates and adsorbed gases. ${ }^{55}$ The objective of substrate cleaning is to remove these chemical impurities and particles from the semiconductor surface to promote a clean epitaxial growth. There are various methods like plasma etch, (dry and wet) chemical etch, flashing etc., to achieve a clean substrate. Based on the type of substrate and 
growth temperature requirement, we use a modified approach of wafer cleaning based on aqueous-chemical processes as described below.

\subsubsection{IMEC/Shiraki cleaning}

All $\mathrm{Si}(001)$ substrates were chemically cleaned using a modified $\mathrm{IMEC}^{56} /$ Shiraki $^{57}$ cleaning process unless otherwise stated. This involves a $\mathrm{H}_{2} \mathrm{SO}_{4}+\mathrm{H}_{2} \mathrm{O}_{2}$ (2:1) mixture to remove organic materials from $\mathrm{Si}$ surface, such as hardened polymer resists and other contaminants of organic nature. For inorganic contaminants, a buffered oxide etch (HF), followed by a $\mathrm{H}_{2} \mathrm{O}$ (DI) $+\mathrm{HCl}+\mathrm{H}_{2} \mathrm{O}_{2}$ (4:1:1) heated solution, with subsequent buffered oxide strip is performed multiple times. A final etch with concentrated $\mathrm{H}_{2} \mathrm{O}(\mathrm{DI})+\mathrm{HCl}+$ $\mathrm{H}_{2} \mathrm{O}_{2}$ (2:4:1) mixture creates a passivating suboxide $\left(\mathrm{SiO}_{\mathrm{x}}\right)$ layer. The passivated wafers were rinsed, dried, then fixed on a molybdenum sample holder using ceramic tweezers, then transferred through a load-lock chamber into the MBE growth chamber. The cleaning sequence is attached in Appendix 2. The passive $\mathrm{SiO}_{\mathrm{x}}$ layer was desorbed in situ at $800{ }^{\circ} \mathrm{C}$ after an overnight temperature ramp, plus $5 \mathrm{hr}$. prebake at $600{ }^{\circ} \mathrm{C}$, resulting in a clean $2 \mathrm{x} 1$ reconstructed Si surface for epitaxial growth.

The cleaning sequence mentioned above requires a process temperature of $800{ }^{\circ} \mathrm{C}$ for oxide desorption, which degrades a pre-imposed lithographic pattern due to high temperature processing, see Figure 2-4. For our directed self-assembly research, we adopted a low temperature cleaning process to preserve the patterns. This low temperature cleaning process involves the IMEC/Shiraki clean sequence, as explained earlier, followed with a quick dip in buffered HF. The HF dip strips the passive oxide formed by IMEC/Shiraki clean and then creates a passive $\mathrm{H}$ - terminated Si surface. This process was based on the work by Thompson ${ }^{58}$ and Eaglesham ${ }^{59}$. The passive H-termination layer was desorbed in 
situ at $550{ }^{\circ} \mathrm{C}$ after an overnight temperature ramp, plus $5 \mathrm{hr}$. prebake at $400{ }^{\circ} \mathrm{C}$, resulting in a clean $2 \times 1$ reconstructed Si surface for epitaxial growth.
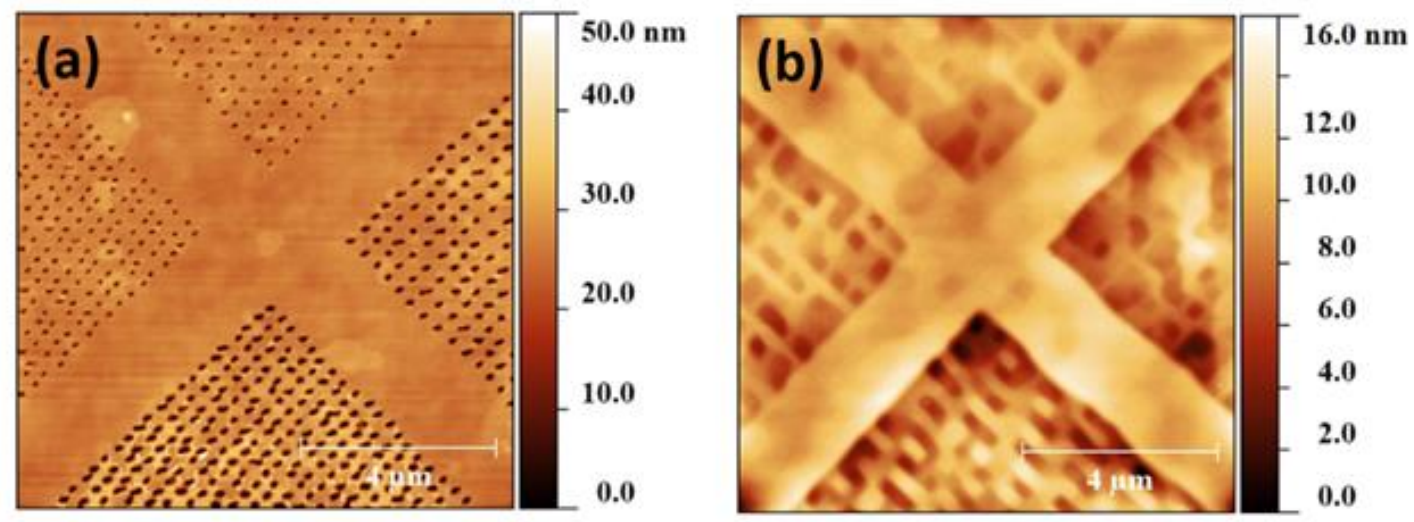

Figure 2-4: (a) 10x10 $\mu^{2}$ AFM height image showing patterned region prior to the insertion to MBE. (b) $10 \times 10 \mu \mathrm{m}^{2}$ AFM height image of the same area as shown in (a) post $800{ }^{\circ} \mathrm{C}$ desorb cycle in $\mathrm{MBE}$ - no Si buffer deposited.

\subsubsection{Ultraviolet/ozone cleaning}

The handling and cleaning processes prior to insertion into the MBE for $\mathrm{Ge}(001)$ substrates were different compared to the $\mathrm{Si}$ substrate. $\mathrm{Ge}(001)$ substrates were ultrasonically precleaned in successive rinses of trichloroethylene (TCE), acetone and isopropyl alcohol (IPA). They were then rinsed in DI water to dissolve the native oxide followed by ozone exposure in an ultraviolet (UV) lamp enclosure for rapid regrowth of clean germanium oxide. The process of DI water and UV-ozone exposure was repeated several times with the last step being the UV-ozone exposure. ${ }^{60,61}$ The oxide layer was desorbed in situ at about $450{ }^{\circ} \mathrm{C}$ after a $5 \mathrm{hr}$ prebake at $350{ }^{\circ} \mathrm{C}$, resulting in a clean $2 \times 1$ reconstructed $\mathrm{Ge}$ surface for epitaxial growth. 


\subsection{Reflection high-energy electron diffraction (RHEED)}

Throughout the deposition process in MBE, the Si and Ge surface was monitored in situ using reflection high-energy electron diffraction (RHEED), which gives a real-time characterization of epitaxial growth and crystalline surface quality. A high-energy (10-30 $\mathrm{keV}$ ) electron beam at a glancing angle $\left(<5^{\circ}\right)$ towards a substrate is used to probe the surface of the substrate, see Figure 2-5 showing the schematics of the RHEED setup. Even though RHEED is operated at higher energies the penetration depth is in the order of $<10$ $\mathrm{nm}$ due to the glancing angle setup, therefore making it a surface sensitive technique. The forward scattered electrons from the surface are viewed on a phosphor screen, which is recorded by a charge coupled device (CCD) camera. (Note: Surface diffraction occurs when the Ewald sphere intersects with the reciprocal lattice rods).

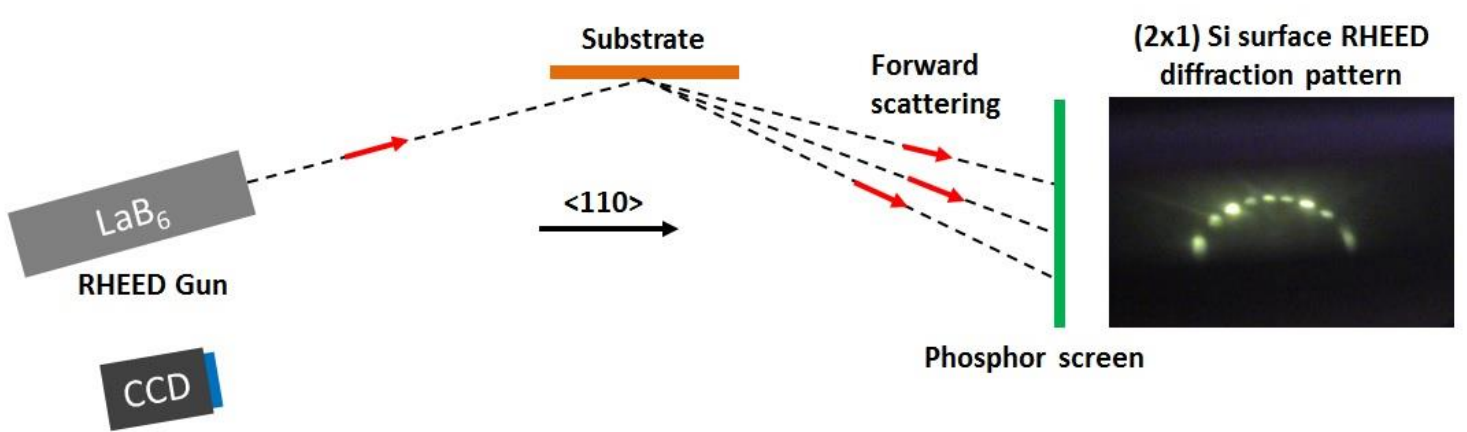

Figure 2-5: Schematic of the RHEED setup in the MBE chamber showing a RHEED pattern recorded from a $\mathrm{Si}(001)(2 \times 1)$ surface along a $\langle 110\rangle$ direction.

Alignment of the substrate azimuth is critical to achieve the appropriate diffraction condition for the RHEED setup. For $\mathrm{Si}(001)$ and $\mathrm{Ge}(001)$ substrates, the electron beam is typically aligned along a $\langle 110\rangle$ direction. Types of diffraction patterns recorded along the $<110>$ direction are shown in Figure 2-6. By monitoring the RHEED pattern as shown in Figure 2-6, we get evidence about the surface quality/ condition during epitaxial growth as 
well as during in situ desorb/ cleaning stages. It should be noted that the incident beam diameter on the sample surface is in the order of $1 \mathrm{~mm}$ and thus gives the surface information on a macroscopic lateral scale.
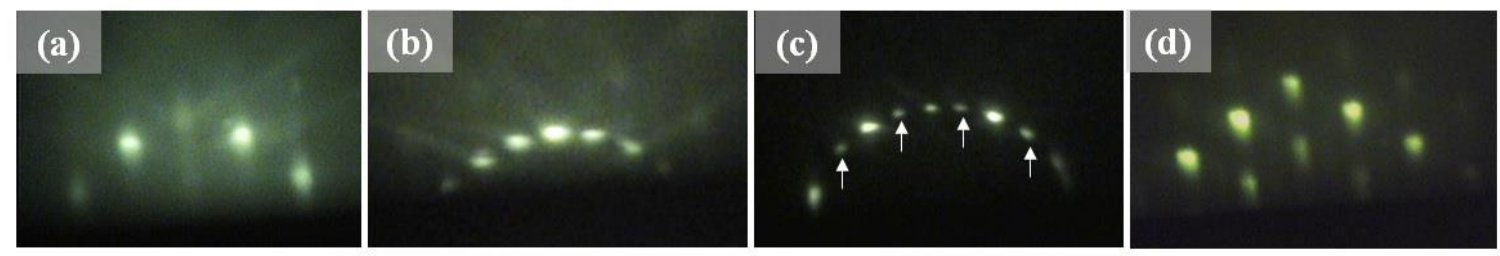

Figure 2-6: RHEED patterns of $\mathrm{Si}(001)$ along $<110\rangle$ : (a) diffuse 2D pattern prior to desorb cycle, (b) first order and (c) second order Laue spots indicating a (2x1) surface reconstruction of $\mathrm{Si}$, (d) atomically rough 3D pattern, showing diffraction through 3D QDs.

\subsection{Atomic force microscopy (AFM)}

Surface morphology for all our samples was characterized ex situ using atomic force microscopy (AFM) with a NT-MDT Solver Pro in tapping mode, using NSG01/NSG10 tips with a typical tip curvature radius of $6 \mathrm{~nm}$.

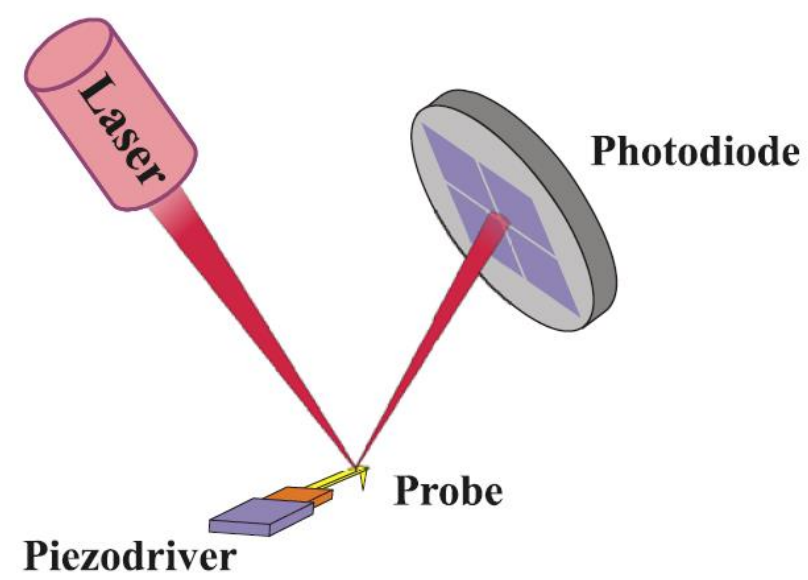

Figure 2-7: Layout of an atomic force microscopy (NTMDT solver pro AFM manual)

AFM is a surface analysis technique that allows the topographical measurement of all surface morphological nanostructures. Topographic imaging of the samples was accomplished by operating the AFM in tapping (semi-contact) mode where the tip/probe 
is oscillated at a resonating frequency and changes of the tip amplitude is measured, see Figure 2-7. The intermittent contact with the sample during tapping mode causes the change in tip oscillation due to surface interactions. A piezoelectric drive adjusts the tip height by raising it away from the sample in order to maintain the tip-oscillation amplitude. In this way, the tip can be rastered across the sample and the z-height is recorded. Figure 2-8 shows typical AFM micrographs displaying a smooth $\mathrm{Si}(001)$ surface post $\mathrm{Si}$ buffer growth along with a much rougher Si surface with 3D faceted QDs on the surface.
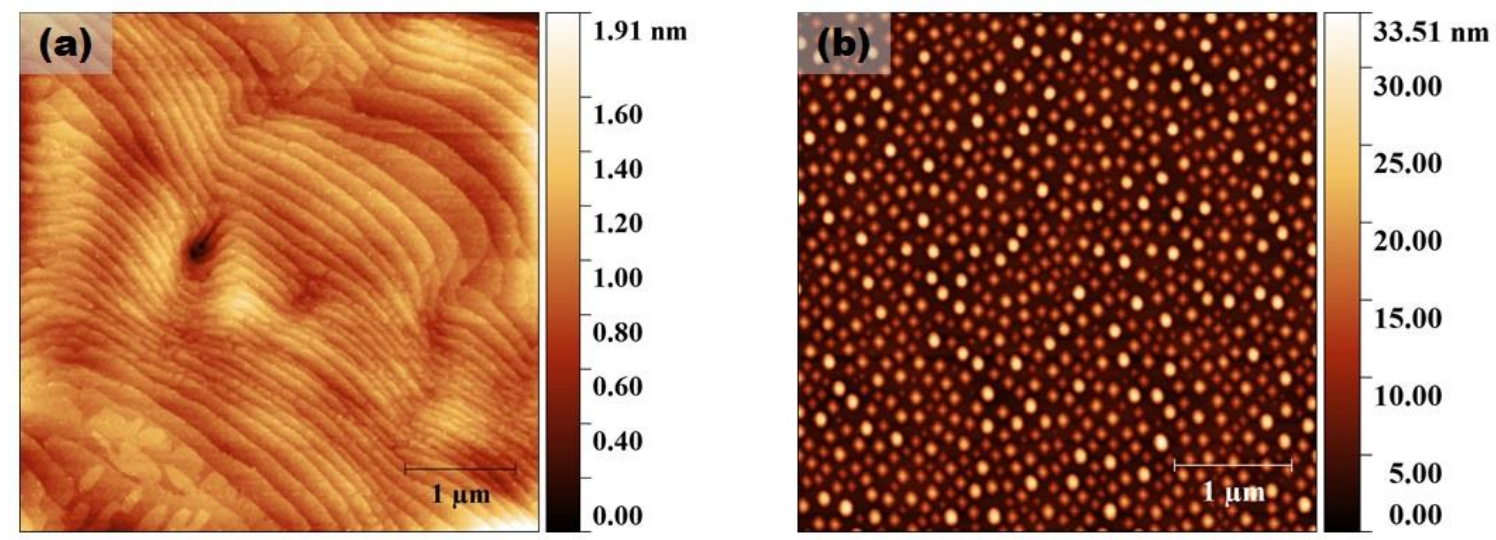

Figure 2-8: AFM micrograph of $\mathrm{Si}$ (001) showing the surface morphology post (a) $50 \mathrm{~nm}$ of Si buffer layer growth at $700{ }^{\circ} \mathrm{C}$, and (b) $3 \mathrm{~nm}$ of $\mathrm{Si}_{0.5} \mathrm{Ge}_{0.5}$ alloy growth at $650{ }^{\circ} \mathrm{C}$.

\subsection{X-ray diffraction (XRD)}

Strain, thickness and composition of the alloy films were characterized ex situ by x-ray diffraction (XRD) thin-film measurement technique. The XRD instrument used was a Rigaku SmartLab x-ray diffractometer ${ }^{62}$ in the laboratory of Prof. Jiwei Lu, with a $3 \mathrm{~kW}$ copper sealed tube generator. All the measurements were made at a wavelength of $\lambda=$ $1.5406 \AA$ obtained using a parallel beam Ge 220x2-bounce monochromator. A schematic of the XRD setup is shown in Figure 2-9. 


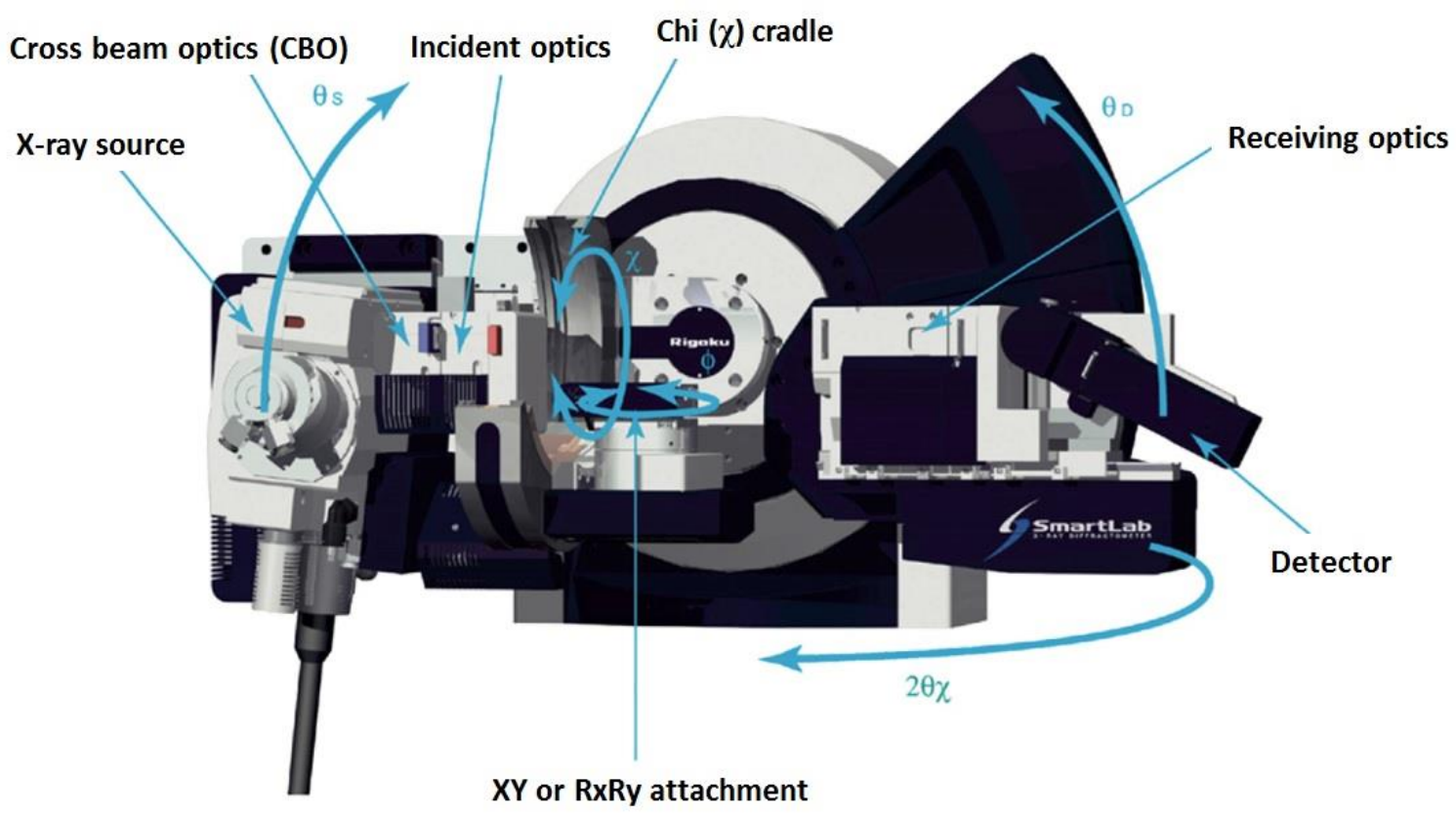

Figure 2-9: Schematic of the Rigaku SmartLab x-ray diffractometer. ${ }^{62}$

As our samples are epitaxial thin films, the XRD measurement technique is different and more difficult compared with measurements of a powder sample. For instance, an epitaxial thin film (single crystal) has a strong preferred orientation such that only one set of specific lattice planes can be detected. Therefore, even a slight misalignment of the incident optics prior to the measurement will result in no observable intensity from the specific lattice
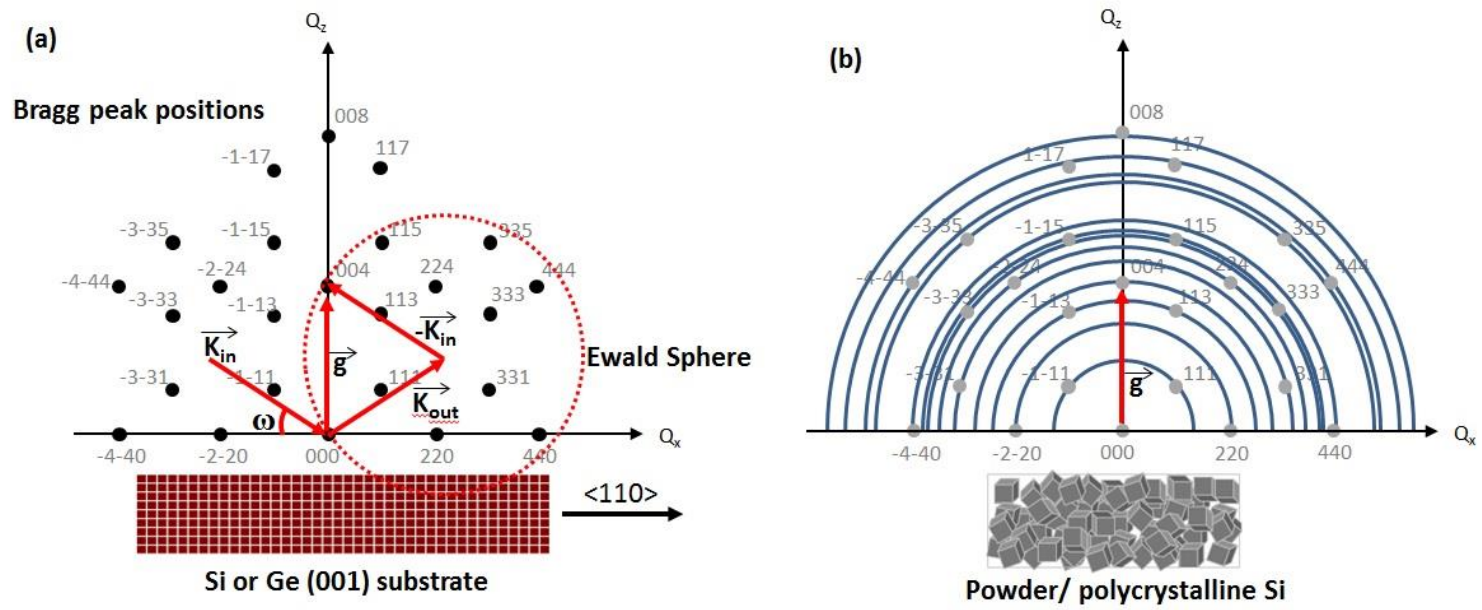

Figure 2-10: (a) Bragg peaks for an epitaxial film (single crystal) (b) Diffraction rings 
planes for epitaxial thin film samples, see Figure 2-10. Note that for single crystal Si(001), a $\omega / 2 \theta$ scan along the $(001)$ azimuth results only in $00 l$ diffraction peak. By comparision, for powder sample, a $\omega / 2 \theta$ scan normal to the surface (which does not need to be in any specific azimuth) results in all possible $h k l$ diffraction peaks. Figure 2-11 shows the reciprocal space map (RSM) of the (004) and (224) peaks of a SiGe film and the Si substrate recorded with XRD. Recording such RSM maps takes a long time therefore in general only a slice from the RSM is recorded during the data collection of XRD process with various XRD geometry and scan direction.
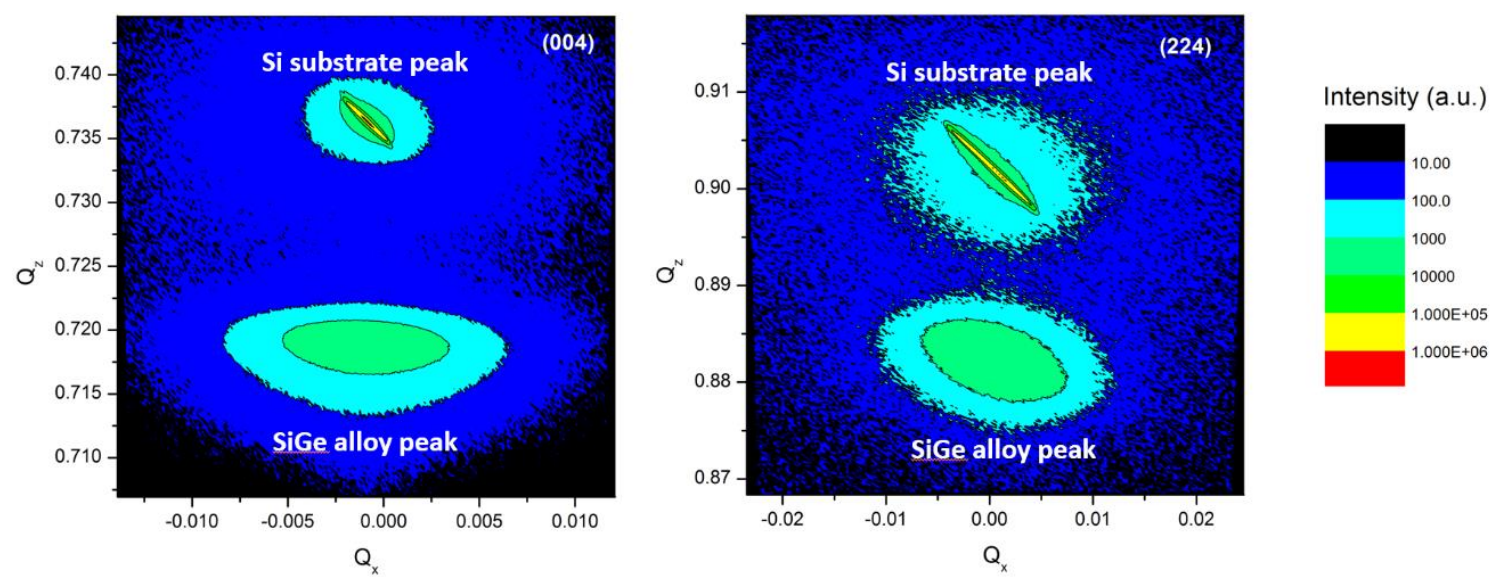

Figure 2-11: Reciprocal space map (RSM) of (a) 004 peak and (b) 224 peak for $300 \mathrm{~nm}$ thick $\mathrm{Si}_{0.5} \mathrm{Ge}_{0.5}$ alloy grown on $\mathrm{Si}(001)$ substrate. $\mathrm{Q}_{\mathrm{x}}$ and $\mathrm{Q}_{\mathrm{z}}$ are the reciprocal space units along <110> and <001> respectively. ( $Q_{z}$ for $(224)$ scan is along <224> however it has been normalized along <001> for comparison with (004) RSM scan)

For the alignment of the XRD optics prior to the measurements of our sample, two sequences of alignment were routinely performed. First, an automated sample alignment was performed by the SmartLab software which coarsely aligns the sample stage and the XRD optics. In this automated alignment process the software simply adjusts the height of sample stage with a direct X-ray beam. After the alignment, the sample surface and incident X-ray beam are essentially parallel. Second, a manual alignment of $\omega / 2 \theta$ is performed 
where the incident optics is aligned with the detector. In our case, as we grow on $\mathrm{Si}(001)$ and $\mathrm{Ge}(001)$ substrates, we take the (004) diffraction peak from substrate as the reference for manual alignment. A series of $\omega, \omega / 2 \theta$ and $2 \theta$ scan is performed to align the incident and receiving optics manually; more on the alignments procedure can be found in Rigaku Journal ${ }^{63}$. Figure 2-12 shows the schematics of three of the most commonly used scan direction in reciprocal space.

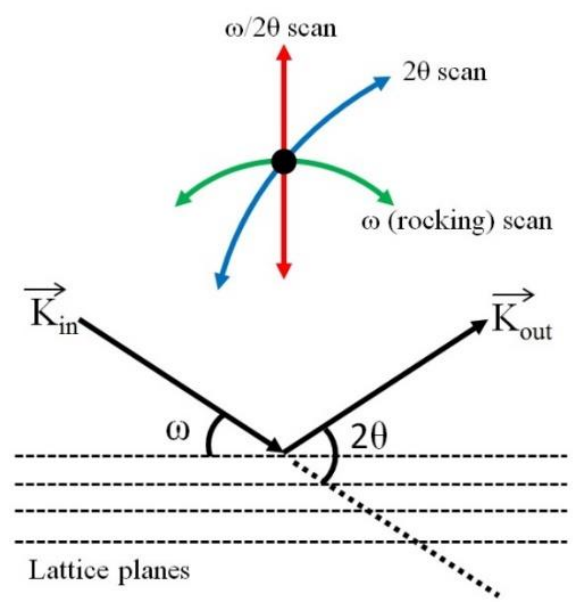

Figure 2-12: Schematics of XRD scanning geometry and direction.

\subsubsection{Lattice parameter, composition and strain characterization}

Ge composition " $\mathrm{x}$ " and the strain in the films can be determined by measuring the 004 and 224 reflections from the sample via conventional " $\omega$-2 2 " and rocking curve $(\omega)$ scans in XRD. ${ }^{64,65}$ Figure 2-13 shows a typical data recorded for 004 and 224 peaks from a conventional $\omega-2 \theta$ scan. The SiGe alloy peak shift from the Si substrate is due to strain in the film; for compression the shift is towards the left and for tension the shift is towards the right of the substrate/ bulk peak. Extracting lattice constants, strain and composition from such XRD scan is complicated as strain is coupled to unrelaxed lattice constants of the film. 

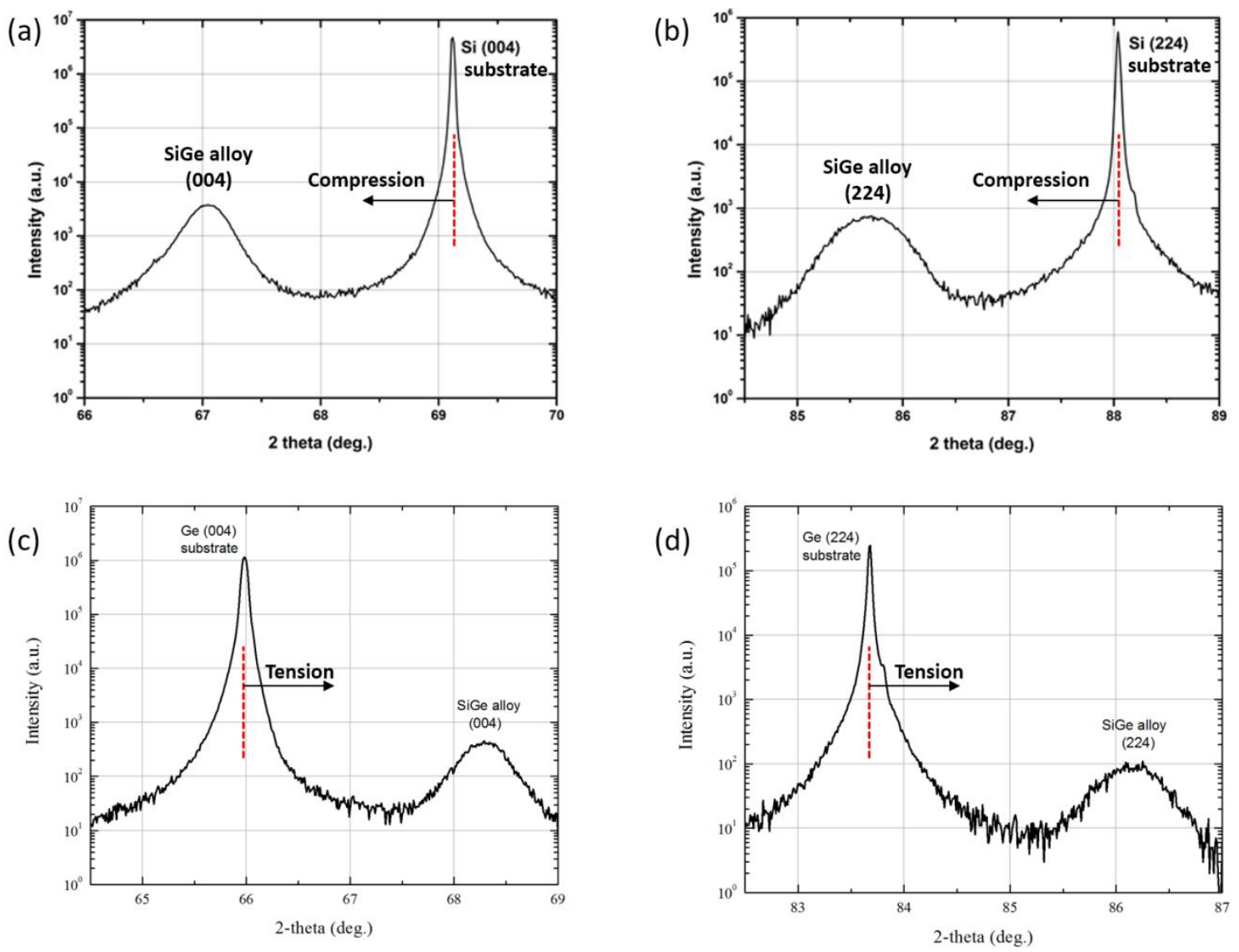

Figure 2-13: $\omega$-2 $\theta$ scan of (004) and (224) peak for $100 \mathrm{~nm}$ thick $\mathrm{Si}_{0.5} \mathrm{Ge}_{0.5}$ alloy deposited on $\mathrm{Si}(001)$ substrate: (a), (b) and $\mathrm{Ge}(001)$ substrate: (c), (d).

Here, we will further discuss our approach to decouple and calculate the strain and composition of the film. The lattice parameter for an unstrained $\mathrm{Si}_{1-\mathrm{x}} \mathrm{Ge}_{\mathrm{x}}$ alloy film is given by $^{64,66}$

$$
\mathrm{a}_{\mathrm{SiGe}}=5.4309+0.200326 \mathrm{x}+0.026274 \mathrm{x}^{2}
$$

Therefore, in order to calculate the composition " $\mathrm{x}$ ", from Equation 2-1, we will need to experimentally calculate the relaxed lattice parameter of the film (asiGe) first. With an assumption of pseudomorphic growth (tetragonal distortion of a SiGe layer deposited on Si or Ge substrate) of SiGe alloy film, the relaxed lattice parameter of the layer is related 
to the in-plane $\left(\mathrm{a}_{\mathrm{II}}\right)$ and perpendicular $\left(\mathrm{a}_{\perp}\right)$ lattice constant of the SiGe alloy film and is given by

$$
\mathrm{a}_{\text {SiGe }}=\frac{(1-v)}{(1+v)} \times \mathrm{a}_{\text {SiGe }}^{\perp}+\frac{2 v}{(1+v)} \times \mathrm{a}_{\text {SiGe }}^{\mathrm{II}}
$$

where $v=$ Poisson ratio: $v_{\mathrm{Si}}=0.278$ and $v_{\mathrm{Ge}}=0.271 .{ }^{64}$ To obtain the perpendicular lattice constant $(\mathrm{a} \perp)$, we performed a symmetric $(\omega-2 \theta)$ scan with substrate $(004)$ plane parallel to the surface. Simply derived from Bragg's law - $a_{\perp}$ is given by

$$
\mathrm{a}_{\perp}=\frac{2 \lambda}{\sin \left(\theta_{004}^{\mathrm{Si}}+\Delta \omega_{004}\right)}
$$

where $\lambda=$ wavelength of $\mathrm{Cu} k \alpha_{1}$ radiation (1.5406 $\AA$ ), $\theta_{004}^{\mathrm{Si}}=$ Bragg angle for (004) Si peak $=34.565^{\circ}$ and $\Delta \omega_{004}=$ angular spacing between the substrate peak and the constant $\mathrm{Ge}$ composition layer peak. For in-plane lattice constant (aII), we performed an asymmetric ( $\omega$ - 20) scan with substrate (224) plane parallel to the surface. From Bragg's law

$$
\mathrm{a}_{\mathrm{II}}=\frac{\sqrt{2} \lambda}{\sqrt{\left(\mathrm{a}_{\mathrm{SiGe}}^{\perp}{ }^{2}\right) \sin ^{2}\left(\theta_{224}^{\mathrm{Si}}+\Delta \omega_{224}\right)-4 \lambda^{2}}} \mathrm{a}_{\mathrm{SiGe}}^{\perp}
$$

where $\theta_{224}^{\mathrm{Si}}=$ Bragg angle for (224) Si peak $=44.015^{\circ}, \Delta \omega_{224}=$ angular spacing between the substrate and the constant Ge composition layer peak, and $\mathrm{a}_{\mathrm{SiGe}}^{\perp}=$ perpendicular lattice parameter deduced from Equation 2-3. With experimentally determined values of $a_{\perp}$ and 
aII, asiGe can be calculated from Equation 2-2 and then the composition from Equation 2-1.

Further, the degree of strain relaxation for our SiGe alloy films is given by,

$$
R=\frac{\left(a_{S i G e}{ }^{I I}-a_{S i}\right)}{\left(a_{S i G e}-a_{S i}\right)}
$$

\subsubsection{Thickness measurement}

The thickness of the alloy film was characterized by x-ray reflectivity (XRR) measurement.

It is a technique to analyze a grazing x-ray reflection beam in order to measure thickness, density and surface/interface roughness of the thin film. When an x-ray beam is incident at
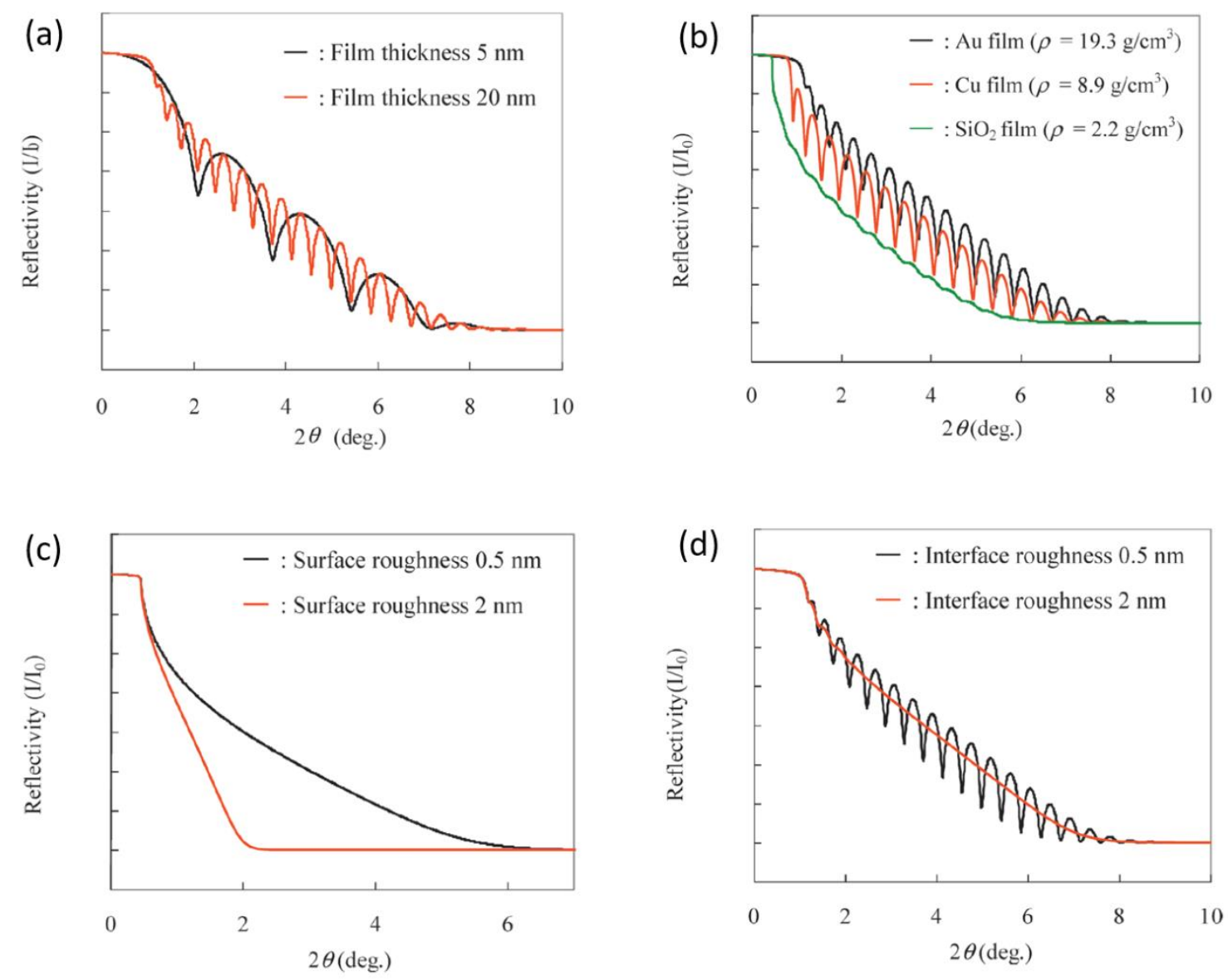

Figure 2-14: X-ray reflectivity curve of: (a) Au film on Si substrate with different thickness, (b) $\mathrm{Au}, \mathrm{Cu}$ and $\mathrm{SiO}_{2}$ film on $\mathrm{Si}$ substrate (film thickness is $20 \mathrm{~nm}$ ), (c) Si substrate with two different values of surface roughness, and (d) Si substrate with different interface roughness (film thickness is $20 \mathrm{~nm})^{142}$ 
a grazing angle on a flat substrate the total reflection of the beam occurs below a critical incident angle. The interference of such a reflected beam from sample surface and the substrate-film interface results in the reflectivity profile with oscillations caused by $\mathrm{x}$-ray interference as shown in Figure 2-14. The oscillations are also referred to as Kiessig fringes, which depend upon film thickness such that thicker the film give shorter oscillation period, see Figure 2-14(a). Apart from just thickness, information like film density, surface

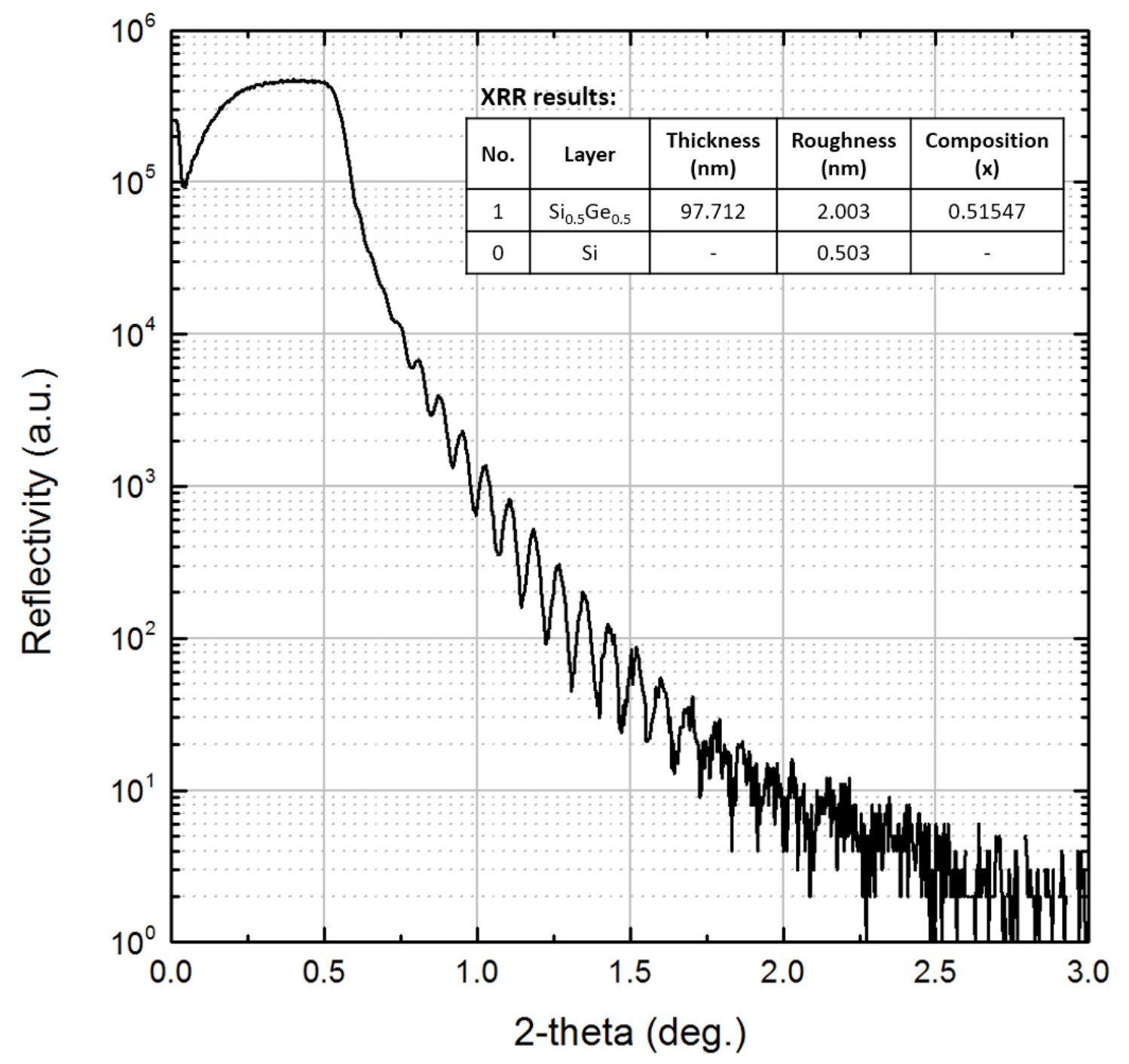

Figure 2-15: XRR data for nominal $100 \mathrm{~nm}$ thick $\mathrm{Si}_{0.5} \mathrm{Ge}_{0.5} / \mathrm{Si}(001)$ sample. The reflectivity curve obtained from sample was analyzed with Rigaku software simulator; extracted results are listed in the table. 
roughness and interface roughness can also be estimated as incident $\mathrm{x}$-ray beam is sensitive to these parameters. The critical angle of incidence and the amplitude of the oscillation fringes are directly related to the density of films such that the larger the film density, the higher is the oscillation amplitude, see Figure 2-14(b). For larger surface roughness, the reflectivity envelope decays more rapidly, see Figure 2-14(c). Further Figure 2-14(d) demonstrates the effect of interface roughness on the reflectivity curve where amplitude of oscillations decreases with increasing interface roughness.

For all growths, $\mathrm{Si}$ and $\mathrm{Ge}$ sputter guns were calibrated to deposit $\mathrm{Si}_{1-\mathrm{x}} \mathrm{Ge}_{\mathrm{x}}$ alloys with $\mathrm{x}=$ $50 \%$. The post-growth Ge composition (x) determined by XRD for all of the samples were found to be $0.47<x<0.53$ and will be referred to as $50 \%$ alloy in this work. A sample plot of the XRR data from one of our sample is given in Figure 2-15.

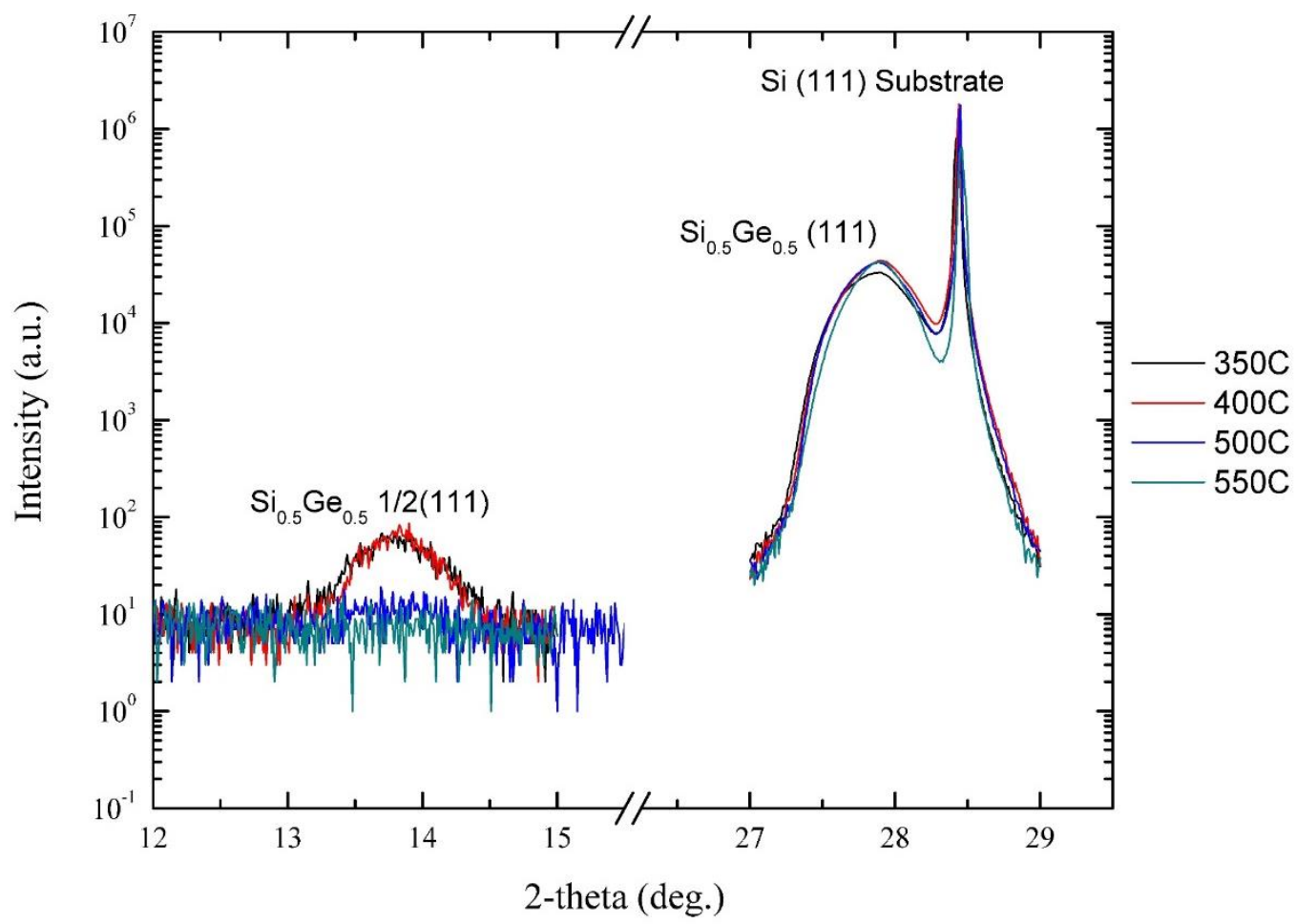

Figure 2-16: Comparison of $1 / 2$ (111) superlattice peak of $300 \mathrm{~nm}$ thick $\mathrm{Si}_{0.5} \mathrm{Ge}_{0.5} / \mathrm{Si}(001)$ grown at various temperature with (111) fundamental alloy peak for order parameter calculation. 
Further, XRD was also routinely used to characterize the order parameter (S) of the sample by comparing the forbidden superlattice integrated intensity: $1 / 2(111)$, to the fundamental reflections: (111), to quantify the long-range order in the sample, which will be discussed in Chapter 3, see Figure 2-16.

\subsection{Electron beam lithography (EBL)}

EBL is a fundamental nanofabrication technique, enabling patterning technologies to create masks and templates with direct writing of structures down to sub-10 nm dimensions. ${ }^{67}$ EBL patterning process involves three major steps: exposure, development and pattern transfer. During exposure a highly focused electron beam is used to modify the solubility of a resist material coated on the substrate. In the development step, the modified portions of resist material are then stripped off, leaving a patterned resist which can then be used as a mask to transfer the pattern to the substrate by etching. A schematic diagram of the patterning process is shown in Figure 2-17.

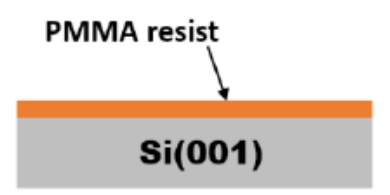

(a) Resist coat

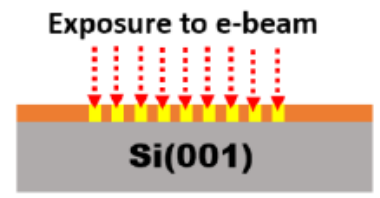

(b) Exposure

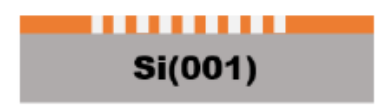

(c) Development

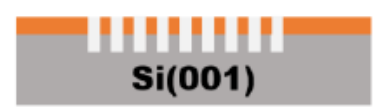

(d) Pattern transfer

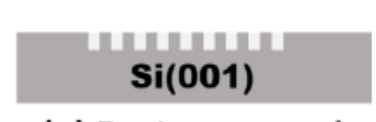

(e) Resist removal

Figure 2-17: Outline of EBL patterning process to form nanoscale pattern with a positivetone resist layer.

In general, there are three common ways to write the patterns via EBL, where the dose can be defined by a pixel, line or an area. In pixel exposure, a dot or pixel is exposed with the e-beam for a well-defined dwell time that controls the dot dose given in $\mathrm{pC} / \mathrm{dot}$. In line 
exposure, the system exposes a single line of pixels in a series to write a line feature where dose is given in $\mathrm{pC} / \mathrm{cm}$. And for area exposure, the system undergoes a series of lineexposures to create an exposed area of defined shape and size and is given in $\mu \mathrm{C} / \mathrm{cm}^{2}$. Figure 2-18(a) shows a dot-pattern with different pitches and doses designed in a pattern generator where dose was defined in a single pixel. Figure 2-18(b) and (c) shows the developed pattern image taken from optical scope and SEM respectively. Dose control is an essential part of EBL patterning process as it controls the final feature size, see Figure 2-18(d).
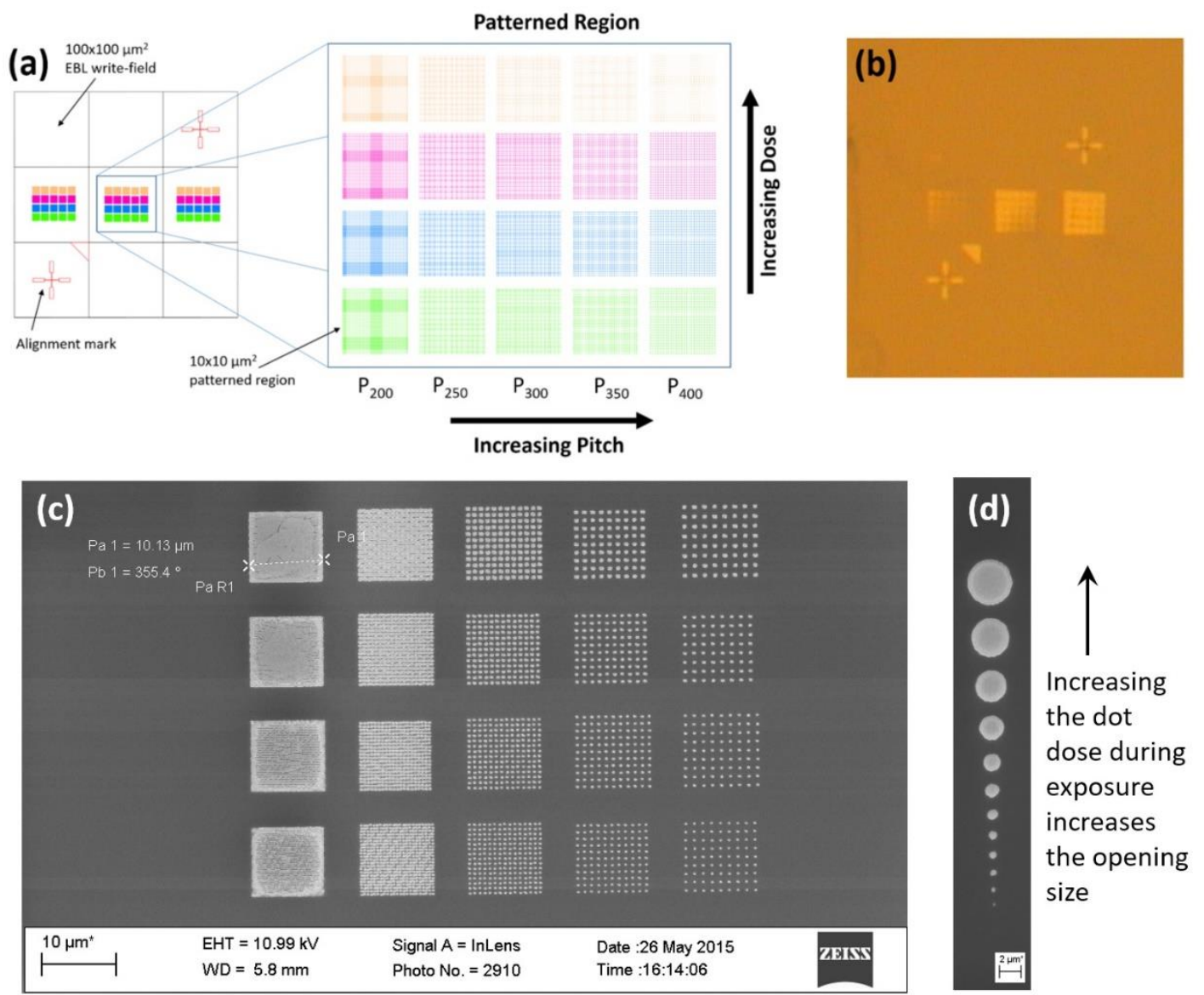

Figure 2-18: (a) Dot-pattern generated with AutoCAD for e-beam exposure, (b) optical image of the patterned region after developing the patterns, (c) SEM image of one of the patterned region, and (d) a series of dot/pixel exposed at increasing dose from bottom to top showing the ability to control the feature size with dose control. 
EBL systems have an advantage of producing very high resolution patterns with the ability to customize the pattern design easily. However, to write large area and complex patterns takes longer time. The resolution or the smallest feature that an EBL system can write depends mostly on the electron source and column optics. Initially, for learning purposes, all the EBL patterning and preliminary testing was performed utilizing Raith-50 EBL at UVa with $\mathrm{LaB}_{6}$ filament source. Even though the Raith-50 was nominally capable of writing features below $50 \mathrm{~nm}$, the uniformity of patterns severely degraded below a pattern pitch of $500 \mathrm{~nm}$, see Figure 2-19. Therefore, all of our higher resolution patterning $(\leq 500$

(a)
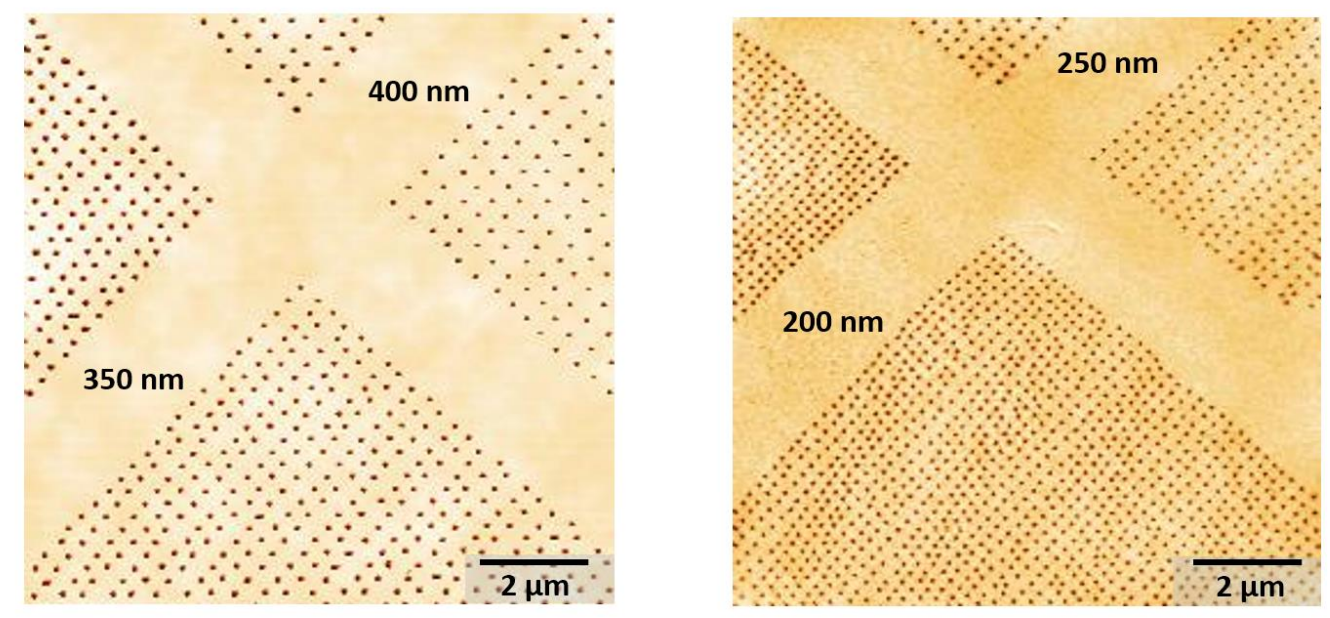

(b)
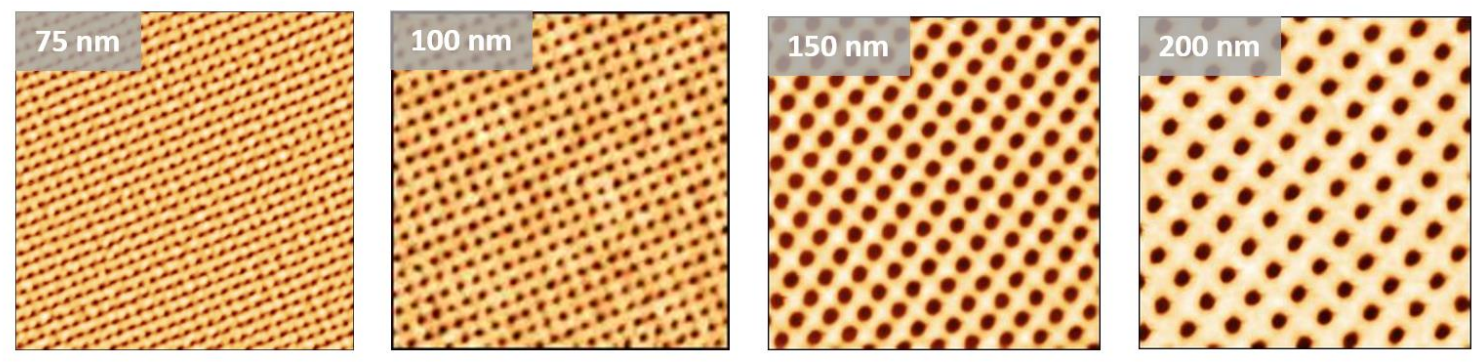

Figure 2-19: (a) Raith-50 at UVa was not optimized to perform patterning with pitch $\leq 500$ $\mathrm{nm}$ where patterns with lower pitch were non-uniform. (b)Pattern pitch 75-200 nm; Raithe-line was able to perform high resolution patterning with better uniformity and precision. 
$\mathrm{nm}$ ) was performed at the University of Maryland - Nanocenter FabLab on a Raith-e-line system with tungsten field emission (FE) source. We performed all the patterning and optimization work in a Raith-e-line independently and the post-patterning process was performed at the University of Virginia Microfabrication Laboratories (UVML) at UVa. More details on the EBL patterning optimization work is presented in Chapter 5.

\subsection{Transmission electron microscopy (TEM)}

Transmission electron microscopy (TEM) was performed on both an FEI Titan 80-300 operated at $300 \mathrm{kV}$ and a JEOL 2000-FX operated at $200 \mathrm{kV}$. In TEM, high energy electron beam is transmitted through an ultra-thin specimen $(<100 \mathrm{~nm})$ where the electrons interact with specimen as it passes through it. Careful interpretation of the transmitted electrons provide insight into structural and compositional configuration of the sample. A diagram

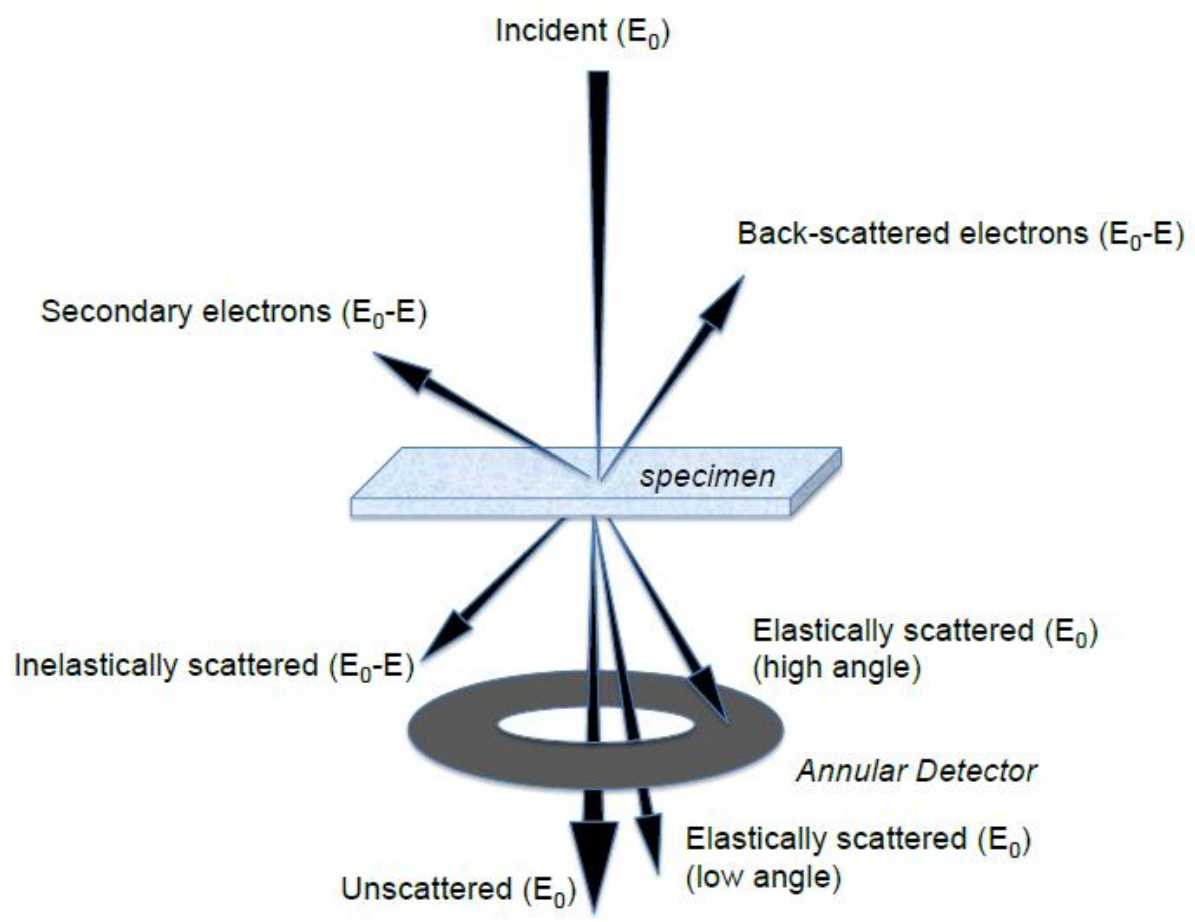

Figure 2-20: A schematic diagram of electron-sample interaction. ${ }^{121}$ 
of electron-sample interactions is shown in Figure 2-20. The forward scattering of electrons at low angles is very important since crystalline materials will diffract the electron beam when the Bragg condition is met. Owing to the small de Broglie wavelength of electrons, the TEM is capable of imaging at significantly higher resolution where even single atomic columns can be resolved, also known as high-resolution transmission electron microscopy (HRTEM). See Figure 2-21(c), which shows a HRTEM image of a Si lattice. For chemically-ordered structures, in addition to the fundamental reflection in TEM
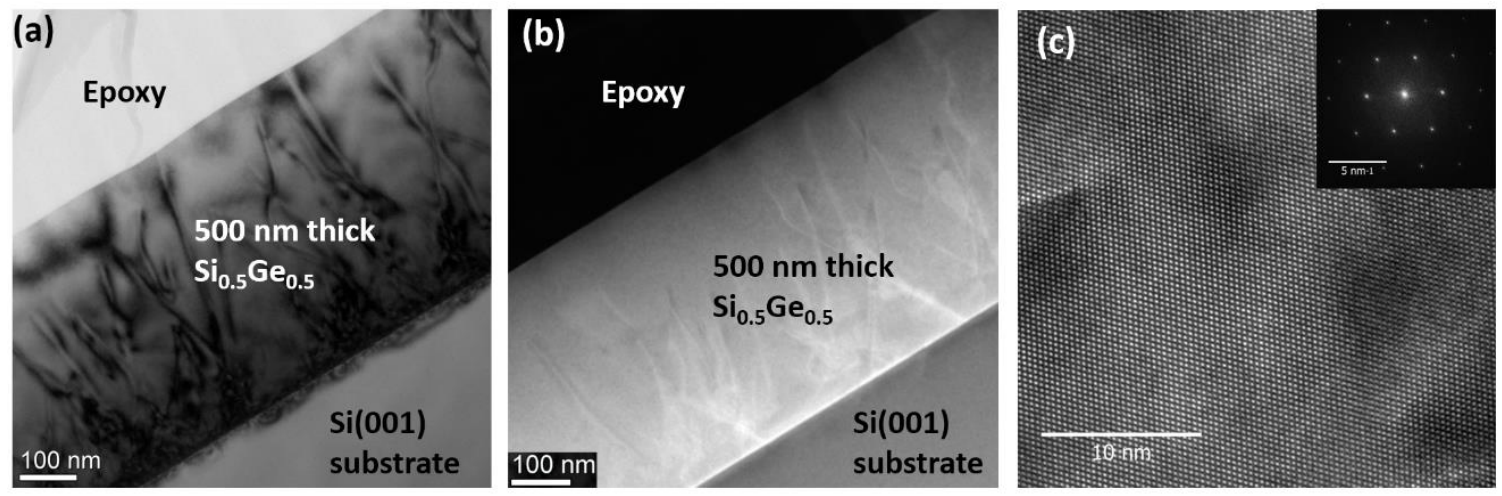

Figure 2-21: TEM images of a $500 \mathrm{~nm}$ thick $\mathrm{Si}_{0.5} \mathrm{Ge}_{0.5} / \mathrm{Si}$ sample (a) BF image, (c) STEM image: Ge having a greater $\mathrm{Z}$ than $\mathrm{Si}$ therefore $\mathrm{SiGe}$ alloy appears as the lighter intensity due to increased high angle scattering, and (c) HRTEM image along with FFT image showing the Si lattice along a $<110>\mathrm{ZA}$.

diffraction, extra reflections also known as forbidden reflections may be present. The intensity of such forbidden reflections can be directly used to quantify the ordering in the material. However, we have used the observation of forbidden reflections from TEM as qualitative analysis method to analyze the ordering in our samples which will be discussed in more detail in Chapter 3. The TEM can also be used in z-contrast imaging mode where scanning transmission electron microscopy (STEM) is incorporated. In STEM mode, a condensed beam is rastered across the sample surface and scattered electrons are collected in an annular detector. Note that in TEM mode the electrons transmit therefore higher Z- 
atoms or any change from regular lattice (due to strain) will give a dark contrast (electrons diffract) whereas in STEM mode contrast is opposite as only elastically scattered electrons at higher angle are collected by the annular detector to form an image, see Figure 2-21. Cross-sectional TEM (XTEM) samples were prepared via both focused ion beam (FIB) lift-out process as well as mechanical thinning, where dimple grinding was used followed by glancing incidence ion milling with $4 \mathrm{keV} \mathrm{Ar}^{+}$. The FIB lift-out process was used when a cross-section from any specific region of the wafer was required. The mechanical process of TEM sample preparation has been well described and documented by our group in the past. $^{68,69}$ For FIB lift-out process, the scanning electron microscopy (SEM) images showing various stages of the sample preparation is given in Figure 2-22. FIB lift-out was performed at Evans Analytical Group (EAG) and Penn State University.
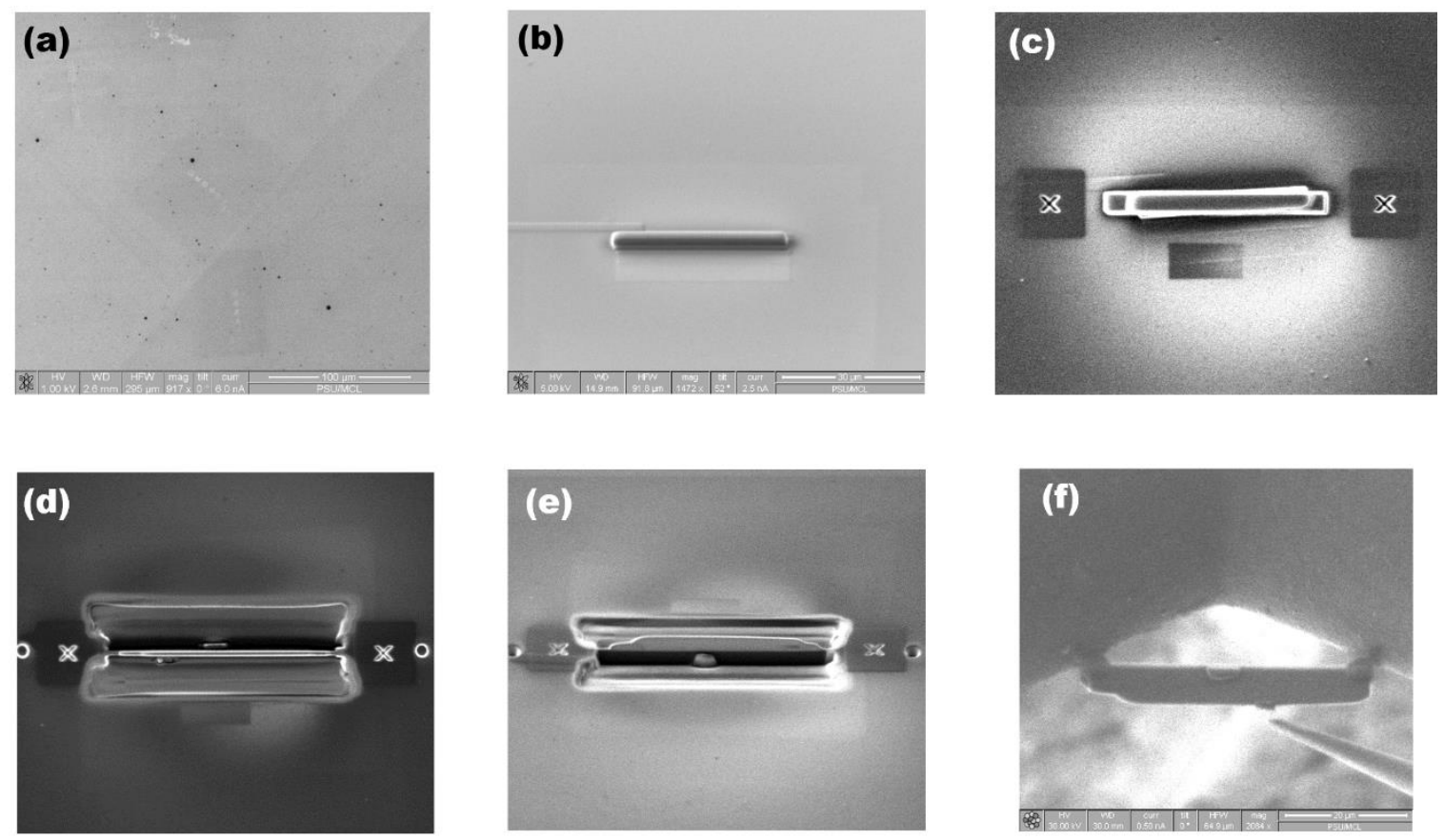

Figure 2-22: SEM images of (a) patterned region from where a TEM cross-section is required, (b) $50 \mu \mathrm{m}$ wide carbon strap is deposited to protect the patterned region on the surface, (c)-(e) sequential milling steps into the Si substrate to expose a sample for lift-out, and (f) thinned sample mounted to half grid for TEM. 


\section{Chapter 3: Mono-layer scale chemically ordered $\mathrm{Si}_{1-\mathrm{x}} \mathrm{Ge}_{\mathrm{x}}$ alloy}

As discussed in Chapter 1, chemical ordering in semiconductor alloys could modify transport properties with potential benefits to thermoelectric application. Initially, the main goal of this work was to grow chemically ordered $\mathrm{Si}_{1-\mathrm{x}} \mathrm{Ge}_{\mathrm{x}}$ alloys and perform transport measurements to investigate the effects of ordering on transport. However, as we will discuss in this chapter, ordering was found both difficult to obtain and to characterize leading us to shift our goals to investigate the growth and ordering mechanism $\mathrm{Si}_{1-\mathrm{x}} \mathrm{Ge}_{\mathrm{x}}$ alloys. Therefore, a parametric study was performed to study how strain, surface roughness, and growth parameters affect the order parameter during the alloy growth which will be discussed below.

\subsection{Ordering in $\mathrm{Si}_{1-\mathrm{x}} \mathrm{Ge}_{\mathrm{x}}$ alloys}

Compositional ordering in $\mathrm{Si}_{1-\mathrm{x}} \mathrm{Ge}_{\mathrm{x}}$ alloys was first reported by Ourmazd and $\mathrm{Bean}^{10}$, where they found additional $1 / 2(111)$ Bragg peaks in the TEM diffraction image of $\mathrm{Si}_{1}$ ${ }_{x} \mathrm{Ge}_{\mathrm{x}} / \mathrm{Si}(001)$ alloy layers. Presence of these weak $1 / 2(111)$ superlattice reflections was attributed to a doubling of the lattice periodicity along a $<111>$ direction. Further, they also proposed that the ordering in these alloys was solely driven by substrate strain. Since this reported observation of ordering in SiGe system, extensive work has been done towards resolving the specific ordered structure at the atomic scale and understanding the detailed ordering mechanisms.

To explain the presence of half-order superlattice reflections, Ourmazd and Bean proposed that the ordered structure to be either RS1 or RS2 (RS = rhombohedral structure), see Figure 3-1. Martins, et al., ${ }^{70}$ and Littlewood ${ }^{71}$ used lattice energy calculations to show that RS2 model is less favorable, and predicted the ordered structure to be RS1. However, 
detailed experimental studies were found to support the existence of RS2 structure and not RS1 as predicted by theoretical calculations. ${ }^{12,72-75}$ (Note: There has been one reported observation of RS1 structure experimentally but their method of characterizing the ordered structure appears to introduce significant error and uncertainty. ${ }^{76}$ ) Tischler, et al., ${ }^{74}$ did show from their detailed XRD study that the ordered structure is more accurately defined by a flexible RS3 structure. The RS3 structure is a generalized version of the (111) ordered phases that is parameterized to be a range of structures between RS1 and RS2. As both RS2 and RS3 represent $\mathrm{L} 1_{1}$-like structure, with alternating $\{111\}$ bilayers enriched in one or the other species with very close structural parameters, the ordered structure will simply be referred to as RS2 here.

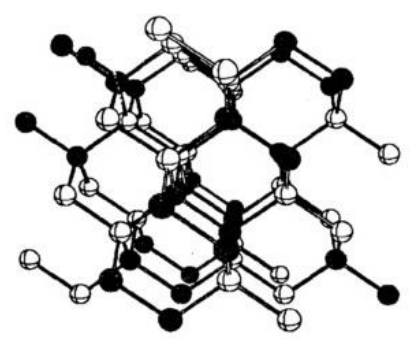

(a) RS1

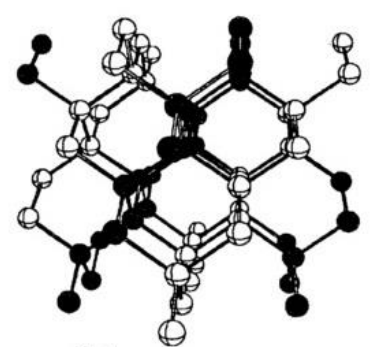

(b) RS2

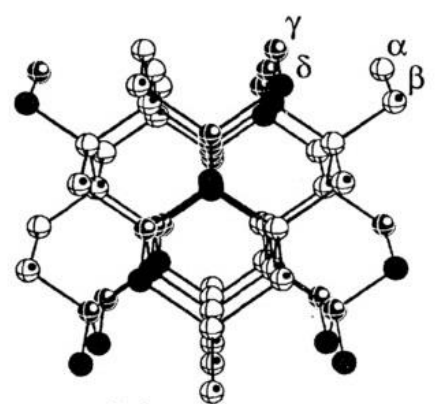

(c) $\mathrm{RS} 3$

Figure 3-1: Proposed rhombohedral structure (RS) models for the ordered structure in $\mathrm{Si}_{1}$ ${ }_{x} \mathrm{Ge}_{\mathrm{x}}$ alloys: (a) RS1 model, (b) RS2 model and (c) RS3 model. View for all the ordered unit cell is along $\langle 110\rangle . \alpha, \beta$ and $\gamma, \delta$ represent Ge-rich and Si-rich sites, respectively. ${ }^{74}$

The experimental confirmations for the existence of RS2 structure were very interesting. Much of the work was then focused on the ordering mechanism, since ordering is generally not stable in the bulk, and to understand why RS2 ordering is preferred. 


\subsection{Ordering Mechanism}

Ourmazd and Bean proposed that the ordering is solely driven by substrate strain where annealing the alloy samples was found to improve the overall ordering. ${ }^{10}$ However, LeGoues, et al., with annealing experiments showed that annealing is not able to improve the ordering; in fact once the ordering is destroyed by high temperature processing further annealing is unable to restore it. ${ }^{72}$ Reported growth temperatures to foster ordering are below $550{ }^{\circ} \mathrm{C}$, generally in the proximity of $400{ }^{\circ} \mathrm{C} .{ }^{10-12,14,15,61,74}$ Kuan, et al., ${ }^{13}$ Muller, et al., ${ }^{11}$ and LeGoues, et al., ${ }^{12,72}$ found that the ordering is not related to substrate strain and its existence is solely dependent upon the conditions of the dynamic growth surface. LeGoues, et al., found that the ordering in Si-Ge alloys occurred only on the 2x1 Si(001) reconstructed growth surface. No compositional ordering was found on SiGe alloys for growth on surfaces other than the $2 \times 1$ dimer-reconstructed $\operatorname{Si}(001)$ surface. Apart from $\mathrm{Si}(001)$ substrates, ordering has been reported for growth on $\mathrm{Ge}(001)$ substrates as well, further corroborating that the epitaxial strain does not drives the ordering process, since the sign of the strain changes for alloy growth on Ge vs. Si. ${ }^{15,61,76}$

With all these observation and reports, it was concluded that the ordering in SiGe is metastable for the bulk of the alloy, and strictly results from surface growth kinetics occurring on the $2 \times 1$ reconstructed growth surfaces. Extensive work has been done to understand the mechanism of RS2 ordering observed in $\mathrm{Si}_{1-\mathrm{x}} \mathrm{Ge}_{\mathrm{x}}$ alloys. However, to date there has been no convincing and direct evidence to support the proposed theoretical models for the origin of ordering. Here we will discuss two of the proposed mechanisms that supports the reported RS2 ordering found in SiGe alloy system; one by LeGoues, et 
al., ${ }^{72}$ and the other by Jesson, et al., ${ }^{77}$ Both the mechanisms are based on surface properties during the growth and not related to bulk thermodynamics.

(a)

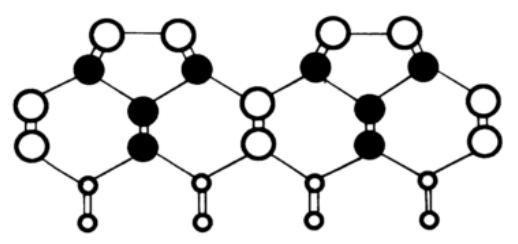

(b)

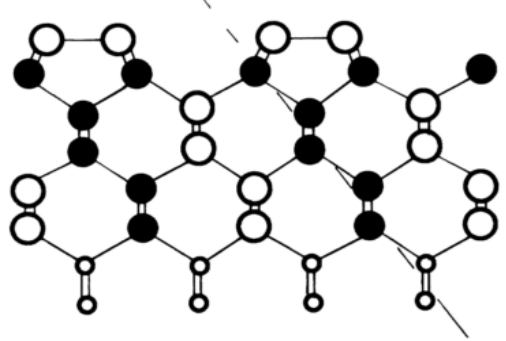

Figure 3-2: (a) Cross-sectional view of a 2x1 dimer surface along a $\langle 110\rangle$. Surface dimers result in the strain underneath the surface resulting in sites under compression favoring $\mathrm{Si}$ occupancy, black circles, and sites under tension favoring Ge occupancy, white circles. (b) Proposed growth model by LeGoues, et al., where growth occurs by the motion of double steps in order to permit large domains along a single $\langle 111\rangle{ }^{72}$

The mechanism proposed by LeGoues, et al., ${ }^{72}$ is based on trapping near-surface equilibrium configurations as the growth surface advances, see Figure $3-2$. The $2 \times 1$ reconstruction is associated with clean $\mathrm{Si}(001), \mathrm{Ge}(001)$ and $\mathrm{Si}_{1-\mathrm{x}} \mathrm{Ge}_{\mathrm{x}}(001)$ surfaces, where the recombination of dangling bonds results in surface dimer formation that introduces large, inhomogeneous strains underneath the surface. Kelires and Tersoff ${ }^{78}$ used Monte Carlo simulation to show that the strain associated with the dimer-reconstruction drives an oscillation of composition in the alloy up to the first four layers from the surface. This oscillation of composition can drive site-specific segregation of $\mathrm{Si}$ and $\mathrm{Ge}$ atoms. Si atoms being comparatively smaller, will migrate to sites under compressive stress while larger Ge atoms will migrate to sites under tensile stress. ${ }^{61,79}$ 
On the other hand, the model proposed by Jesson, et al., ${ }^{77}$ provides a detailed kinetic model, see Figure $3-3$. As the ordering is associated with $2 \times 1$ reconstructed surface, a step-flow atomistic model was suggested at a type- $\mathrm{D}_{\mathrm{B}}$ bilayer step ${ }^{80}$ containing a kink acting as a sink for adatoms. The model requires that the growth surface is Ge-rich (all dimers are $\mathrm{Ge}$ ) due to efficient surface segregation. To propagate the $D_{B}$ kink by one unit requires that four atoms, two $\mathrm{Si}$ and $\mathrm{Ge}$, be added. The two $\mathrm{Si}$ atoms attach at the intermediate (one

(a)

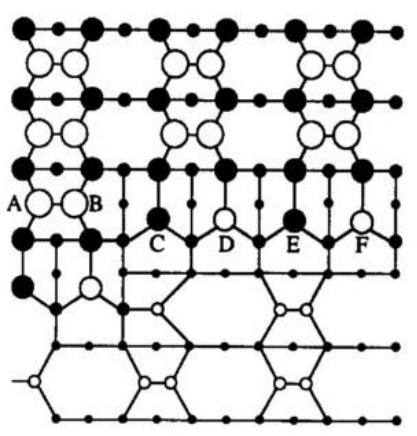

(b)

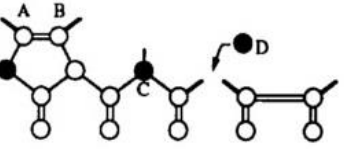

(c)

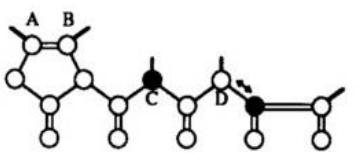

(d) (e)

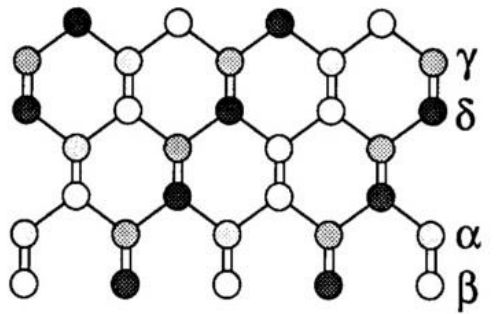

Figure 3-3: Proposed growth model by Jesson, et al., with growth-induced compositional ordering by kink flow growth at $\mathrm{D}_{\mathrm{B}}$ steps. (a) Plan-view of a $2 \times 1$ dimer surface with kink where, $\mathrm{A}$ and $\mathrm{B}$ are surface dimers, and $\mathrm{C}$ to $\mathrm{E}$ represent rebonded edge atoms at the step edge. (b) to (e) shows how the growth takes place by the Ge-pump mechanism resulting in the ordered structure where $\alpha, \beta$ and $\gamma, \delta$ represent Ge-rich and Si-rich sites, respectively. White circles represent Ge atoms and black circles represent $\mathrm{Si}$ atoms. ${ }^{77}$

monolayer up) sites. During this attachment, the geometry is such that in one of these two sites, the Si has a driving force to place exchange with a Ge (the "atom pump") to reduce dangling bond energy. There is no benefit to a switch in the other site. The two Ge atoms then attach as a dimer on the upper step edge, representing ideal surface segregation. Any loss of efficiency in the segregation and pump mechanisms then leads to less than ideal 
long range order, or even a different ordered structure whose structure factor provides a signature for the specific structure.

It is very important to realize that both the ordering mechanisms above require the propagation of double-height steps during growth in order to establish long-range order. However, Araki, et al., ${ }^{81}$ argued that the LeGoues model can also be explained with ML by ML growth model without requiring double-height steps, see Figure 3-4. Ordering in the $\mathrm{Si}-\mathrm{Ge}$ system is not limited to thin films - ordering has also been reported in $\mathrm{SiGe}$ strain-induced 3D islands. ${ }^{82-86}$ The ordering in these self-assembled islands are different

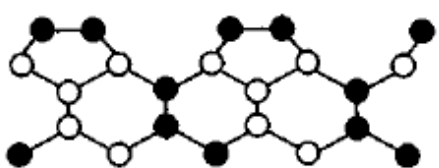

(a)

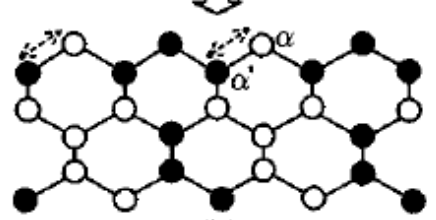

(b)

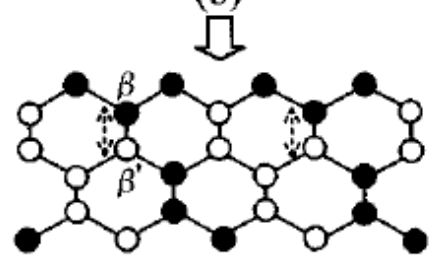

(c)

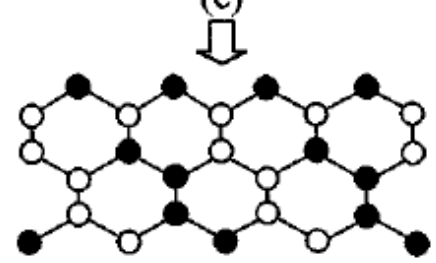

(d)

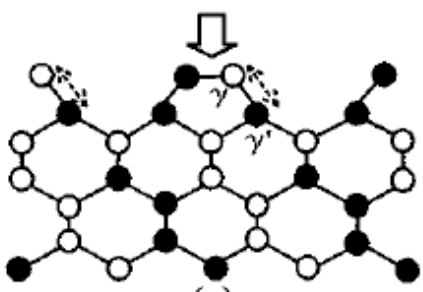

(e)

1.

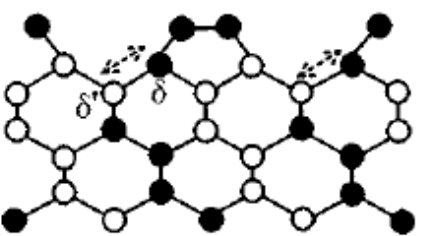

(f)

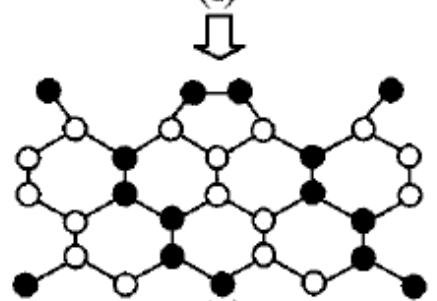

(g)

$\circ \mathrm{Si} \bigcirc \mathrm{Ge}$

Figure 3-4: Surface stress induced ordering mechanism proposed by LeGoues, et al., and revised by Araki, et al., showing ML by ML growth process of the ordering 
and are believed to be driven by strain under some specific facet orientations, but the mechanisms are even less understood than in films.

Proper quantification of the order parameter in these SiGe alloys is very important for comparison work. Surprisingly, only a few papers have accurately and correctly quantified the order parameter in these alloys. ${ }^{61,74}$ In the following section we describe in detail how the order parameter was quantified for all the SiGe samples used here.

\subsection{Order Parameter Considerations}

An order parameter in a binary alloy is defined as $S=\left(f_{A}-F_{A}\right) /\left(1-F_{A}\right)$, where $F_{A}$ is the fraction of species $A$ in the alloy and $f_{A}$ is the fraction of species $A$ on the correct site. ${ }^{87}$ The typical method of measuring an order parameter is to compare the ratio of the integrated peak intensities of a superlattice reflection to that of a fundamental reflection obtained, e.g., via XRD. The integrated intensity measured from XRD, depends upon various factors; based on kinematic diffraction theory the intensity of a reflection from the hkl reciprocal lattice point can be defined as ${ }^{74,88,89}$

$$
I_{\mathrm{hkl}}^{\mathrm{i}}=I_{0} \frac{\mathbf{r}_{\mathrm{e}}^{2} \lambda^{3} \mathrm{~F}_{\mathrm{hkl}}^{2}}{2 \mu \mathrm{V}_{\mathrm{uc}}^{2}} \frac{1+\cos ^{2} 2 \theta_{\mathrm{hkl}}}{\sin 2 \theta_{\mathrm{hkl}}}\left[1-\exp \left(\frac{-2 \mu \mathrm{t}}{\sin \theta_{\mathrm{hkl}}}\right)\right] \mathbf{v}_{\mathrm{i}}
$$

where $\mathrm{I}_{0}$ is the intensity of incident $\mathrm{x}$-ray, $\mathrm{r}_{\mathrm{e}}$ is the classical electron radius, $\lambda$ is the $\mathrm{x}$-ray wavelength, $\mathrm{t}$ is the film thickness, $\mathrm{V}_{\mathrm{UC}}$ is the volume of unit cell, $\mu$ is the linear absorption coefficient and $F_{h k l}$ is the structure factor amplitude. We have explicitly incorporated another factor, $\mathrm{v}_{\mathrm{i}}$, important either when multiple phases are present (e.g., disordered and ordered phases) or when multiple equivalent spatial variants of the ordered phase can occur. The quantity $v_{i}$ is the fraction of the volume illuminated by the $\mathrm{x}$-rays that 
contributes to the hkl diffracted beam, and the index refers to the $\mathrm{i}^{\text {th }}$ spatial variant. For L1 1 -like ordering in a [001] epitaxial film, $\mathrm{i}=1-4$, i.e., there are four spatial variants along the four unique <111> directions, see Figure 1-1. For a typical system undergoing ordering, $v_{i}=1$ for a fundamental reflection, while for a superlattice reflection, $v_{i} \leq 1$ represents the volume fraction of the ordered spatial variant. Typically, the order parameter, $\mathrm{S}$, is determined by measuring the intensity ratio of a superlattice reflection (h'k'l') to a fundamental reflection (hkl). For the superlattice reflection, we can explicitly write $F_{h^{\prime} k^{\prime} l^{\prime}}=S \Phi_{h^{\prime} k^{\prime} l^{\prime}}$, where $S$ is the order parameter, and $\Phi_{h^{\prime} k^{\prime} l^{\prime}}$ is a combination of atomic scattering factors evaluated at the appropriate value of $\sin \theta_{\mathrm{h}^{\prime} \mathrm{k}^{\mathrm{\gamma}} \mathrm{r}} / \lambda$. Then the ratio of intensities can be written as, ${ }^{87,89}$

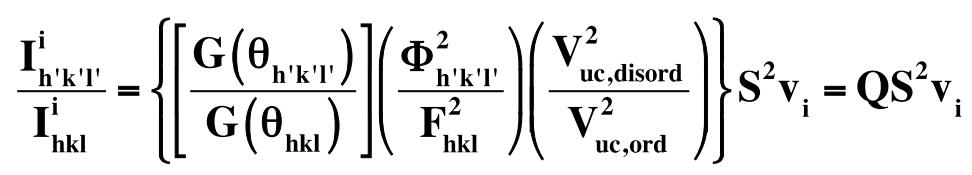

In Equation 3-2, the G's contain the explicit angle-dependent factors in Equation 3-1, and it acknowledges that the volume of the primitive unit cell for the ordered phase need not be the same as the disordered phase. Then for simplicity, we lump all the known physical quantities inside the curly brackets into the parameter Q. Solving for the order parameter, we get

$$
S_{\text {eff }}=S \sqrt{v_{i}}=\sqrt{\left(\frac{1}{Q}\right) \frac{I_{h^{\prime} k^{\prime} \prime^{\prime}}^{i^{i}}}{I_{h k l}^{i}}}
$$

Many researchers who measure the ratio of superlattice-to-fundamental intensities quote the result as the true order parameter, $\mathrm{S}$, when in fact it is the effective order parameter, $\mathrm{S}_{\mathrm{eff}}$ that they measure. For most experiments, it is difficult to separate the true order 
parameter of the ordered region from the volume fraction that is ordered. For example, an effective order parameter $S_{\text {eff }}=0.5$ could correspond to all the material having $S=0.5$, or one quarter of the material having $S=1$. Thus one needs other information, e.g., whether the ordering transition is higher order, or else independent measurements of the volume fraction of ordered phase are required, e.g., by dark-field TEM. In situations where a change in lattice symmetry accompanies ordering, it can be possible to measure both the true order parameter and the volume fraction of the ordered phase. ${ }^{90}$

(a)

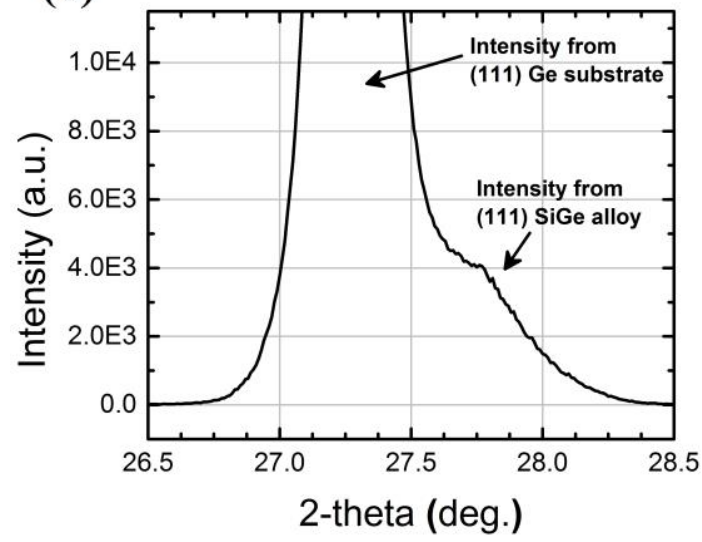

(b)

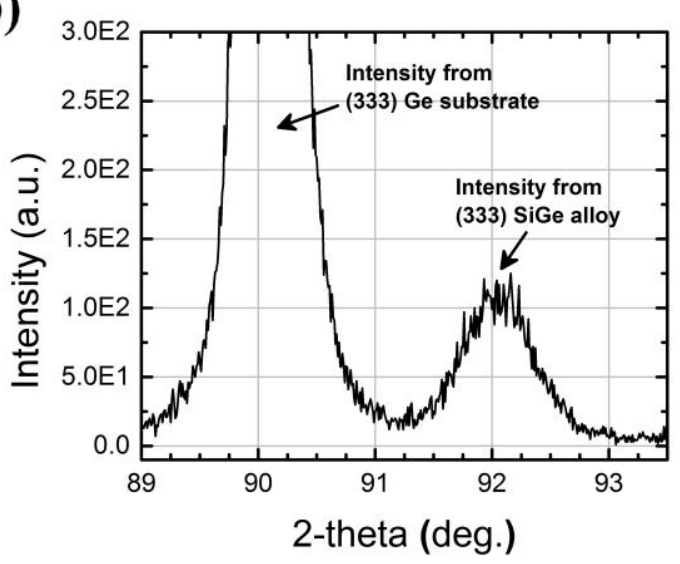

Figure 3-5: $\omega$-2 $\theta$ scan for $100 \mathrm{~nm}$ thick $\mathrm{Si}_{0.5} \mathrm{Ge}_{0.5} / \mathrm{Ge}(001)$, (a) (111) azimuth scan showing significant alloy peak overlapping with the substrate peak, and (b) (333) azimuth scan showing distinct alloy peak resulting in lesser peak-fitting errors.

For all films analyzed here, the integrated intensity from the $1 / 2(111)$ reflection was used for the superlattice reflection. For the fundamental reflection both (111) and (333) integrated peak intensities were used to calculate the order parameter independently and averaged to reduce the peak fitting errors. This was especially useful for samples grown on Ge substrates, where the (111) reflections from the film and substrate have significant overlap, see Figure 3-5. Since these are single-crystal measurements, each choice of a 
superlattice-fundamental pair of reflections only characterizes ordering along one of the four equivalent $\langle 111\rangle$ directions.

The structure factor amplitudes were calculated from the RS2 model, consisting of 64 atoms in a conventional (pseudo-cubic) unit cell. Figure 3-6, shows that good agreement is obtained between our calculated superlattice structure factor values with the Tischler, et. al. ${ }^{74}$, model. The normalized structure factor value used in Tischler, et al.,'s model has been rescaled for absolute scale comparison. Slight variation in the two calculations shown in Figure 3-6, is due to the different sources used for the atomic scattering factor during the structure factor calculation. The factors involved in the calculation of order parameter are

Table 3-1: Factors involved in the calculation of order parameter.

\begin{tabular}{|c|c|c|c|}
\hline \multirow{3}{*}{\multicolumn{2}{|c|}{ Angle }} & $\theta 1 / 2(111)=$ & $6.91^{\circ}$ \\
\hline & & $\theta(111)=$ & $13.94^{\circ}$ \\
\hline & & $\theta(333)=$ & $46.29^{\circ}$ \\
\hline \multicolumn{2}{|c|}{ Linear Absorption coefficient for $\mathrm{Si}_{0.5} \mathrm{Ge}_{0.5}$} & $\mu=$ & $251.3 \mathrm{~cm}^{-1}$ \\
\hline \multirow{3}{*}{\multicolumn{2}{|c|}{$\begin{array}{l}\text { Lorentz-Polarization Factor } \\
\mathrm{f}(\theta)\end{array}$}} & $\theta 1 / 2(111)=$ & 8.122 \\
\hline & & $\theta(111)=$ & 3.808 \\
\hline & & $\theta(333)=$ & 1.003 \\
\hline \multirow{3}{*}{\multicolumn{2}{|c|}{ Structure Factor $|\mathrm{F}|$}} & $|\mathrm{F}| 1 / 2(111)=$ & 522.10 \\
\hline & & $|\mathrm{F}|(111)=$ & 866.17 \\
\hline & & $|F|(333)=$ & 535.43 \\
\hline \multirow{9}{*}{$\begin{array}{c}\text { Absorption Factor } A(\theta) \\
f(\theta, t)\end{array}$} & \multirow{3}{*}{$\mathrm{t}=100 \mathrm{~nm}$} & $\mathrm{~A}(\theta) \frac{1}{2}(111)=$ & 0.0408 \\
\hline & & $\mathrm{A}(\theta)(111)=$ & 0.0206 \\
\hline & & $\mathrm{A}(\theta)(333)=$ & 0.0069 \\
\hline & \multirow{3}{*}{$\mathrm{t}=300 \mathrm{~nm}$} & $\mathrm{~A}(\theta) \frac{1}{2}(111)=$ & 0.1176 \\
\hline & & $\mathrm{A}(\theta)(111)=$ & 0.0606 \\
\hline & & $\mathrm{A}(\theta)(333)=$ & 0.0206 \\
\hline & \multirow{3}{*}{$\mathrm{t}=500 \mathrm{~nm}$} & $\mathrm{~A}(\theta) \frac{1}{2}(111)=$ & 0.1882 \\
\hline & & $\mathrm{A}(\theta)(111)=$ & 0.0990 \\
\hline & & $\mathrm{A}(\theta)(333)=$ & 0.0341 \\
\hline \multirow{2}{*}{ Unit Cell Volume } & Ordered Phase & $\mathrm{V}_{\mathrm{uc} \text {, ord }}=$ & $84.89 \AA^{3}$ \\
\hline & Disordered Phase & $\mathrm{V}_{\mathrm{uc} \text {, disord }}=$ & $42.44 \AA^{3}$ \\
\hline
\end{tabular}


tabulated in Table 3-1, where the intensity ratio between a superlattice and fundamental peak is compared.

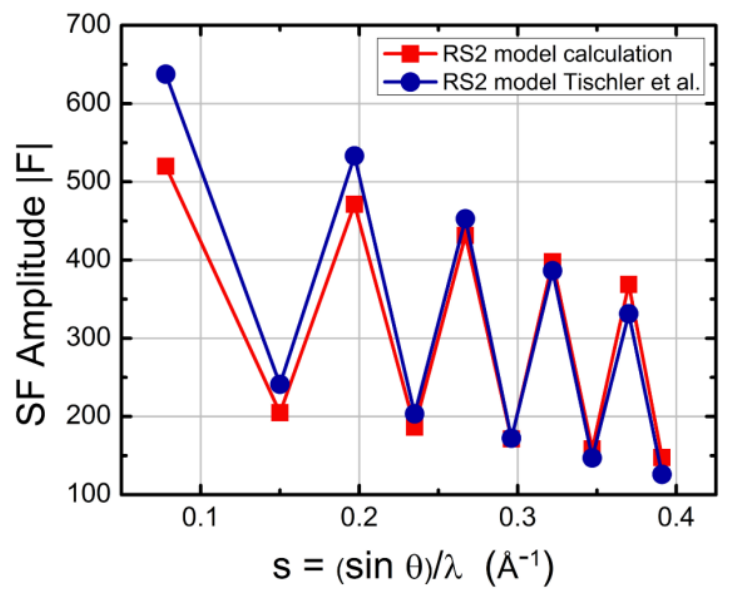

Figure 3-6: Computed structure factor amplitude comparison with that of the RS2 model by Tischler, et al., for the half-order superlattice reflections.

Since the order parameter should strongly affect both thermal and electronic transport, in this work we present a detailed parametric study of how strain, surface roughness, and growth parameters affect the order parameter during the epitaxial growth of $\mathrm{Si}_{1-\mathrm{x}} \mathrm{Ge}_{\mathrm{x}}$ alloys. Our body of data leads to three key results that appear self-contradictory with regard to the role of steps in promoting enhanced ordering.

\subsection{Sample growth and Results}

$\mathrm{Si}_{1-\mathrm{x}} \mathrm{Ge}_{\mathrm{x}}$ alloy thin films were grown on $2 \mathrm{in}$. diameter, $250 \mu \mathrm{m}$ thick, undoped $\mathrm{Si}(001)$ and $\mathrm{Ge}(001)$ wafers. The epitaxial alloy films were grown in our custom, ultra-high vacuum (UHV), hyperthermal molecular beam epitaxy (MBE) system. Details on the MBE and the substrate cleaning process have already been discussed in Chapter 2. For all growths, $\mathrm{Si}$ and Ge sputter guns were calibrated to deposit $\mathrm{Si}_{1-\mathrm{x}} \mathrm{Ge}_{\mathrm{x}}$ alloys with $\mathrm{x}=50 \%$. The postgrowth Ge composition (x) determined by XRD for all of the samples were found to be 
$0.47<x<0.53$ and will be referred to as nominal $50 \%$ alloy in this work. The $\mathrm{Si}_{0.5} \mathrm{Ge}_{0.5}$ alloy was grown on 3 different types of substrate:

(i) Low-miscut $\mathrm{Si}(001)$ substrates,

(ii) Low-miscut Ge(001) substrates, and

(iii) Miscut $\operatorname{Si}(001)$ substrates.

Here, the low-miscut substrate refers to a miscut of $<0.1^{\circ}$. For miscut substrates, miscut angles of $\pm 1^{\circ}$ and $\pm 3^{\circ}$ were used where the miscut direction was specified to be either towards $<100>$ or $<110>$. All the growth substrates were purchased from "Virginia Semiconductor Inc." where the specified miscut was within a tolerance of $\pm 0.1^{\circ}$. This section provides the experimental results, organized by the results of (i) critical growth temperature, (ii) varying the deposition rates, (iii) domain orientation, size and symmetry, (iv) epitaxial strain and growth surface morphology, and (v) comparative MBE grown samples.

\subsubsection{Critical growth temperature}

The first series of samples prepared were $300 \mathrm{~nm}$ thick $\mathrm{Si}_{0.5} \mathrm{Ge}_{0.5}$ alloys on low-miscut, $\operatorname{Si}(001)$ substrates, at growth temperatures: $350,400,450,500$ and $550{ }^{\circ} \mathrm{C}$. For these samples, both the fundamental and superlattice intensities in XRD were found to exhibit a highly symmetric distribution of intensity amongst all the four orientation domains (i.e., the four unique $<111>$ directions). Since the sample has low miscut, we assume that all four spatial ordering variants are equally likely. In this case, $v_{i}=1 / 4$, so that $S=2 S_{\text {eff }}$, and hence we plot the "true" order parameter in Figure 3-7, determined by simple averaging of the intensities over all the four domains. Figure 3-7 shows the results for the dependence of ordering on growth temperature. A growth temperature of $400{ }^{\circ} \mathrm{C}$ exhibited maximum 
$\mathrm{S}$, where the overall ordering sharply decayed at higher growth temperatures. However, overall, the order parameters for these samples were low, with $S_{\max }=0.16$.

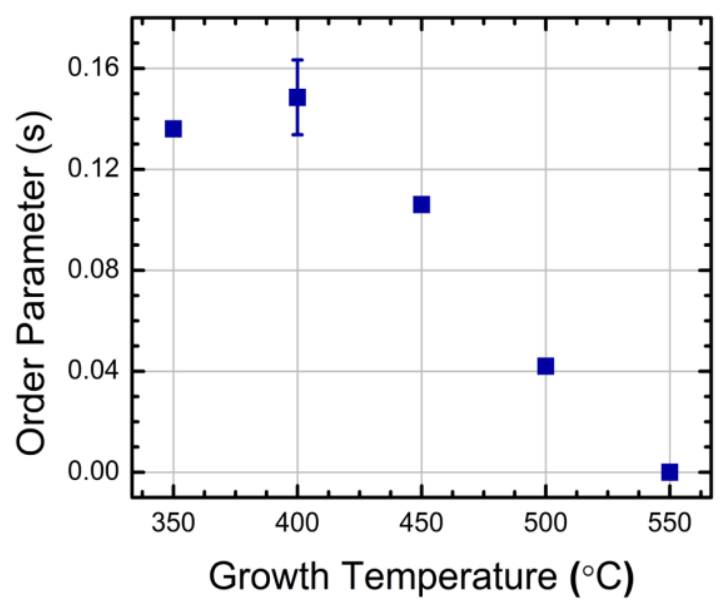

Figure 3-7: Order parameter for $300 \mathrm{~nm}$ thick $\mathrm{SiGe} / \mathrm{Si}$ samples grown at various temperatures. The $400^{\circ} \mathrm{C}$ sample was grown several times to ensure repeatability.

The second series of samples prepared were $100 \mathrm{~nm} \mathrm{Si}_{0.5} \mathrm{Ge}_{0.5}$ alloys on $\mathrm{Si}(001)$ highmiscut substrates (miscut of $\pm 3^{\circ}$ from [001] towards the [100]). As the deliberate miscut on the growth surface breaks the crystal symmetry, the spatial ordered variants are no longer equivalent, so $\mathrm{v}_{\mathrm{i}} \neq 1 / 4$. In such case, we cannot now separate the order parameter and the ordered-domain volume fraction. Therefore, we plot only the "effective" order parameter, $S_{\text {eff. }}$ The effective order parameter vs. the growth temperature for all the 4domains has been plotted in Figure 3-8 (a). The plot clearly shows that the ordering is maximum at $400{ }^{\circ} \mathrm{C}$ with the ordering better in two of the four domains; the asymmetry in the ordering for the four domains is due to the miscut.

The third series of sample prepared were $\mathrm{Si}_{0.5} \mathrm{Ge}_{0.5}$ alloys on low-miscut $\mathrm{Ge}(001)$ substrates (miscut of $<0.1^{\circ}$ ). A SiGe alloy film grown on a $\mathrm{Si}(001)$ substrate is under compression due to lattice mismatch, whereas the same alloy grown on a $\mathrm{Ge}(001)$ substrate is under tension. Similar to the samples grown on $\mathrm{Si}(001)$ substrates, $400{ }^{\circ} \mathrm{C}$ was found to be 
optimal for observing the ordering in $\mathrm{Ge}(001)$ substrates. However, comparing to the samples grown on low-miscut $\mathrm{Si}(001)$, these samples had order parameters about twice as large at $400{ }^{\circ} \mathrm{C}$, as shown in Figure 3-8 (b).
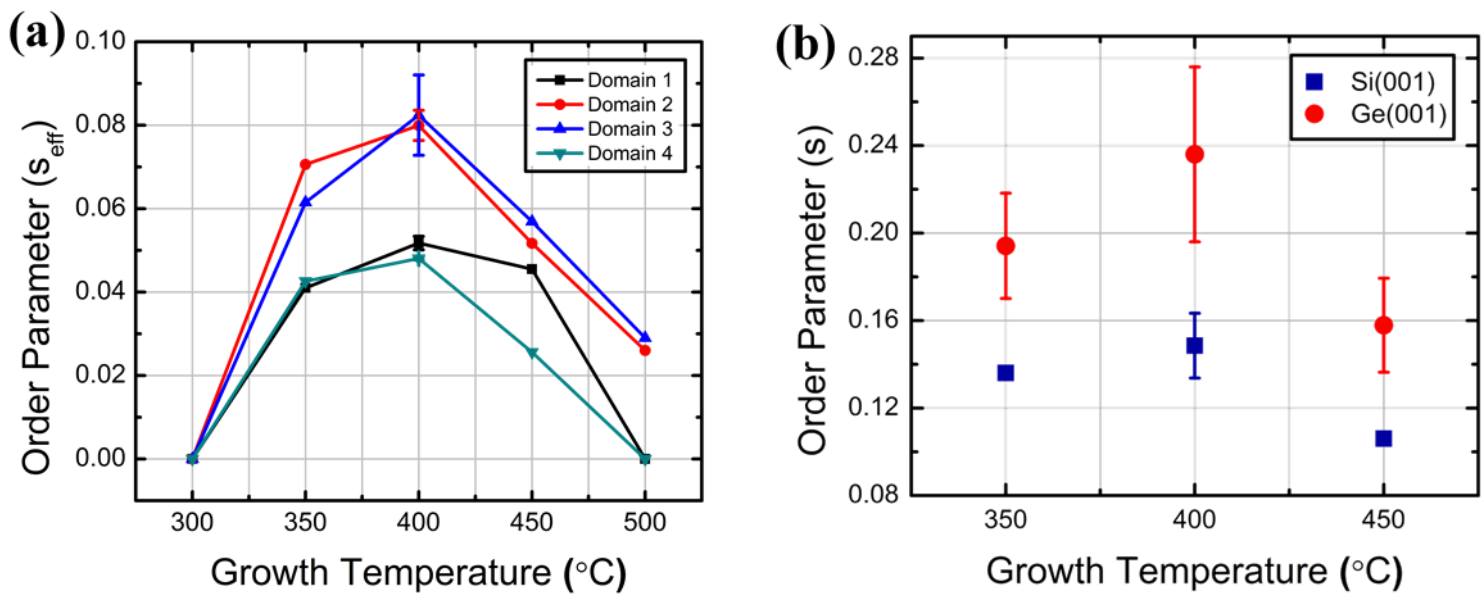

Figure 3-8: (a) Effective order parameter $\left(S_{\text {eff }}\right)$ plotted against growth temperature for $100 \mathrm{~nm} \mathrm{Si}_{0.5} \mathrm{Ge}_{0.5}$ on $3^{\circ}$ miscut $\mathrm{Si}(001)$ substrate, and (b) True order parameter for $300 \mathrm{~nm}$ thick $\mathrm{Si}_{0.5} \mathrm{Ge}_{0.5} / \mathrm{Si}(001)$ and $100 \mathrm{~nm}$ thick $\mathrm{Si}_{0.5} \mathrm{Ge}_{0.5} / \mathrm{Ge}(001)$

\subsubsection{Deposition rate}

Jesson, et al., predicted that ordering could be sensitive to deposition rate. ${ }^{77}$ To explore this, $300 \mathrm{~nm}$ thick $\mathrm{Si}_{0.5} \mathrm{Ge}_{0.5}$ alloys were deposited on $\mathrm{Si}(001)$ substrate with deposition rates: $0.2,0.5,1$ and $2 \AA /$ sec. Since these samples were grown on low-miscut $\operatorname{Si}(001)$ substrates both the fundamental and superlattice intensities in XRD were found to exhibit a symmetric distribution of intensity amongst all four orientation domains. The results showed that there was a slight decay in order parameter as the deposition rate was increased, see Figure 3-9. Similar alloy samples were grown at a deposition rate of $1 \AA / \mathrm{sec}$ several times to test the repeatability and no significant changes were observed. 


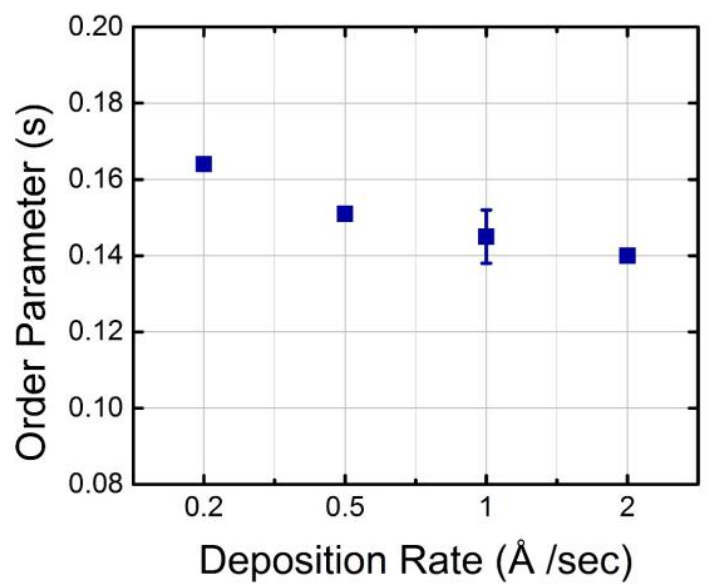

Figure 3-9: Order parameter vs Deposition rate for $100 \mathrm{~nm}$ thick $\mathrm{Si}_{0.5} \mathrm{Ge}_{0.5} / \mathrm{Si}(001)$

\subsubsection{Domain orientation, size and symmetry}

$\mathrm{XRD}$ and TEM techniques were used to analyze the ordered samples for domain orientation, size and symmetry informations. For growth on low miscut $\mathrm{Si}(001)$ substrates, both the fundamental and superlattice intensities in XRD were found to exhibit a highly symmetric distribution of intensity amongst all the four orientation domains (i.e., the four unique $<111>$ directions). It was very hard to extract any quantitative data from the XRD
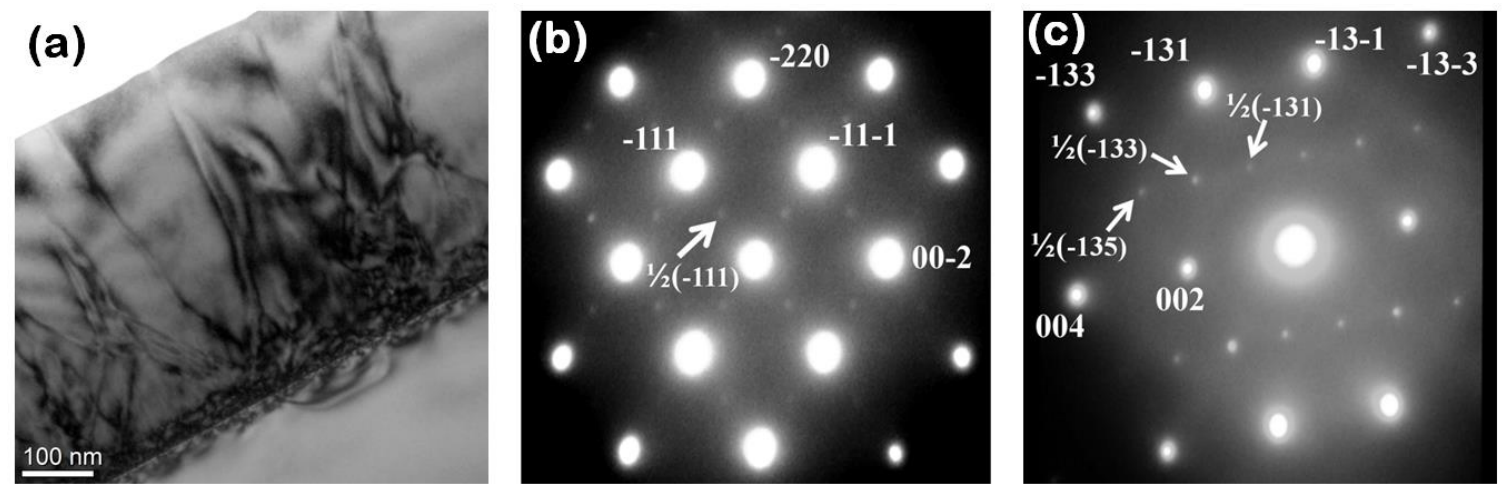

Figure 3-10: (a) TEM bright field cross-sectional image of a $\mathrm{Si}_{0.5} \mathrm{Ge}_{0.5} 500 \mathrm{~nm}$ alloy on $\mathrm{Si}(001)$ low-miscut substrate (b) SAD pattern along [-1-10], and (c) SAD pattern along [-3-10] zone axis showing half-order superlattice diffraction spots. 
regarding the domain size and volume other than symmetry as the superlattice peaks were too weak to perform quantitative analysis.

A subset of samples was examined in XTEM. Although superlattice reflections were observable in selected area diffraction patterns (SAD), these were typically quite weak and difficult to record using a video-based detector, and hence were best imaged in thicker films. Figure 3-10 shows the SAD pattern from a $500 \mathrm{~nm} \mathrm{Si}{ }_{0.5} \mathrm{Ge}_{0.5}$ film on $\mathrm{Si}(001)$ nonmiscut substrates along with its corresponding bright field (BF) micrograph.

The $\langle 310>$ zone axis was found to produce higher superlattice intensities. Extensive threading dislocation content associated with strain relaxation is evident. TEM dark-field (DF) images were also taken for the sample shown in Figure 3-10, with half-order integral superlattice spots taken from $\langle 310\rangle$ zone axis as they were brighter and easier to isolate with an objective apperture in diffraction space. A pair of equivalent superlattice spots were isolated successively and a DF image was recorded to compare and map the two possible domains, see Figure 3-11. It was hard to cleanly resolve the DF image due to weak superlattice intensity and high diffuse scattering. However, $1 / 2(-13-3)$ in part (a) and 1/2(13-3) in part (d) clealy lights up yielding the size of ordered domain in a range of 100-200 nm wide, similar results has been reported by Jesson, et al., ${ }^{14}$ The reason for choosing supperlattice peaks other than $1 / 2(111)$ was because of the size limitation of objective apperture in TEM. Further, the superlattice peaks are more widely spaced in a $<310>\mathrm{ZA}$ making it a lot more easier for the objective apperture to isolate the superlattice spot and record the DF image. 

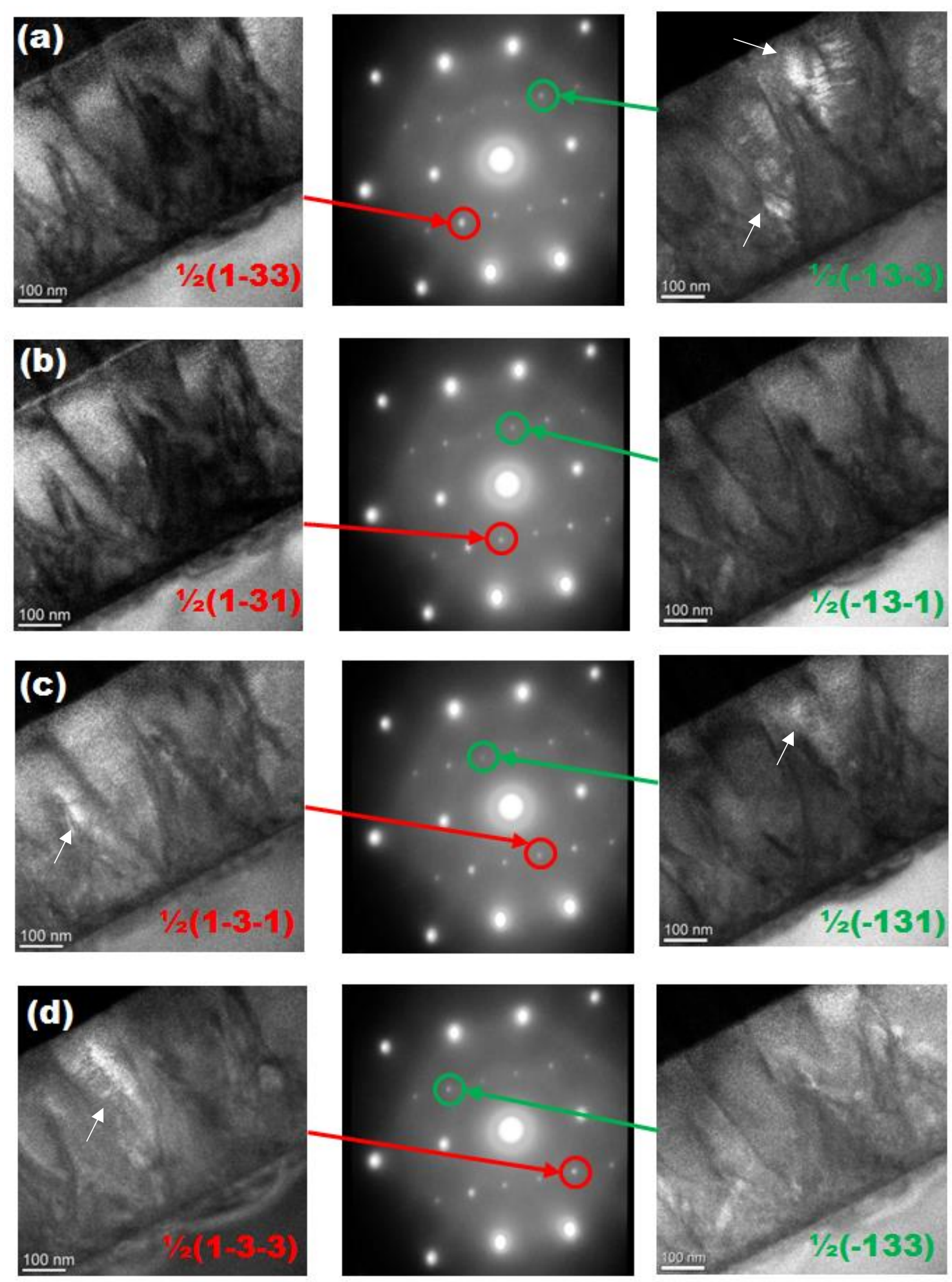

Figure 3-11: DF image taken by placing an OA over the corresponding superlattice peaks as labelled with [-3-10] ZA. The BF image of the sample is shown in Figure 3-10. 
A high-resolution scanning TEM (HR-STEM) image was taken from $500 \mathrm{~nm}$ thick $\mathrm{Si}_{0.5} \mathrm{Ge}_{0.5} / \mathrm{Ge}(001)$ sample, see Figure 3-12. A close observation of the HR-STEM image taken from <110> ZA shows dark and bright bands corresponding to ordered atomic fringes with $\mathrm{Si}$ atoms as dark and Ge atom as bright band. A fast fourier transform (FFT) taken from the HRSTEM image clearly shows the 1/2(111) superlattice peaks. We were able to back track the ordered domain distribution and size by performing a reverse FFT to form
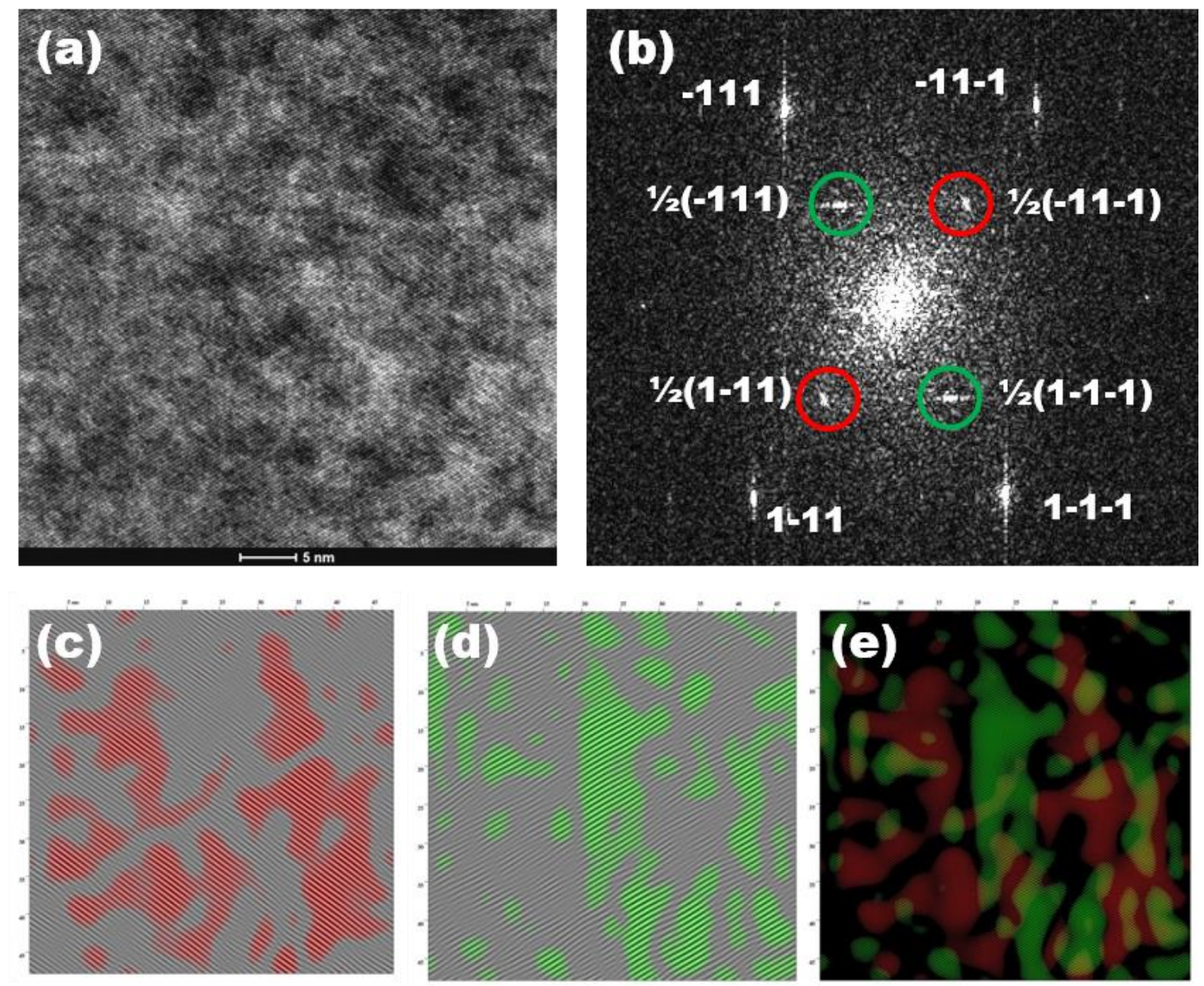

Figure 3-12: (a) HRSTEM image for $500 \mathrm{~nm}$ thick $\mathrm{Si}_{0.5} \mathrm{Ge}_{0.5} / \mathrm{Ge}(001)$ along [110] ZA, (b) FFT image taken from the HRSTEM image, (c) Domain 1 (red) created by performing reverse FFT and taking 1/2(-11-1) and 1/2(1-11) superlattice spots, (d) Domain 2 (green) created by performing reverse FFT and taking $1 / 2(-111)$ and $1 / 2(1-1-1)$ superlattice spots, (e) superimposed image of (c) and (d) 
images from these half integral superlattice spots. The domain size was calculated to be in the range of 20-30 $\mathrm{nm}$ wide.

For growth on miscut substrates the domain size and distribution will be governed by the degree of miscut and substrate symmetry. A series of samples was prepared with $100 \mathrm{~nm}$ $\mathrm{Si}_{0.5} \mathrm{Ge}_{0.5}$ thick alloys on $\mathrm{Si}(001)$ high-miscut substrates (miscut of $\pm 3^{\circ}$ from [001] towards the [100]). Miscut of the (001) substrates changes the step population on the growth surface, see Figure 3-13(a), and growth on the surface miscut towards [100] on the substrate broke the symmetry of the ordered domains along the four variants, see Figure 313(b), suppressing two of the ordered variants. Note that Figure 3-13(b) plots the effective order parameter, $\mathrm{S}_{\mathrm{eff}}$, since we cannot now separate the order parameter and the ordereddomain volume fraction. Figure 3-13(b) also shows results from $100 \mathrm{~nm} \mathrm{Si}{ }_{0.5} \mathrm{Ge}_{0.5}$ alloys
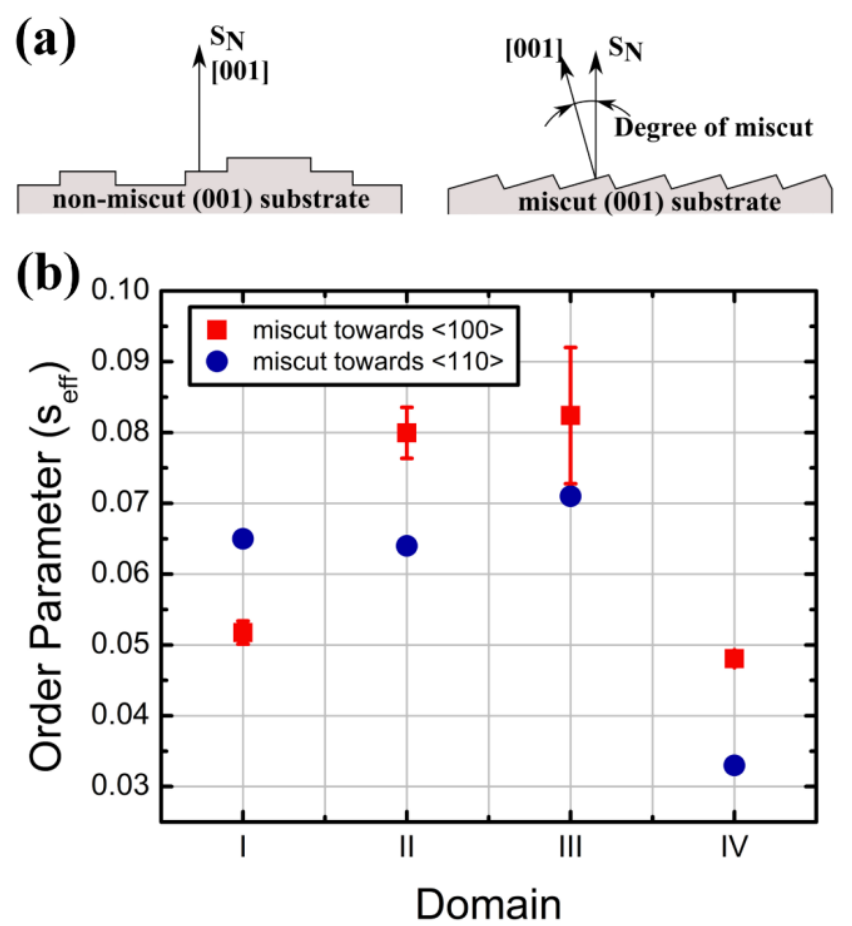

Figure 3-13: Miscut changes the step population on the growth surface; $S_{N}$ is the surface normal (b) Effective order parameter of all four ordered domains, for 100nm thick $\mathrm{Si}_{0.5} \mathrm{Ge}_{0.5}$ alloy on $3^{\circ}$ miscut $\mathrm{Si}$ substrates. 
on $\operatorname{Si}(001)$ substrates with a miscut of $\pm 3^{\circ}$ from [001] towards the [110] direction, where the ordered domain population is suppressed in only one of the variants due to symmetry.

\subsubsection{Epitaxial strain and growth surface morphology}

Due to lattice mismatch, a SiGe alloy film grown on a $\mathrm{Si}(001)$ substrate is under compression, whereas the same alloy grown on a $\mathrm{Ge}(001)$ substrate is under tension. As discussed in Section 3.4.1, the SiGe alloy grown on Ge(001) substrate had superior ordering compared to the alloys grown on $\mathrm{Si}(001)$. To study the strain state of the film and the effect of film thickness on overall ordering, 100, 300 and $500 \mathrm{~nm}$ thick $\mathrm{Si}_{0.5} \mathrm{Ge}_{0.5}$ films were grown on both $\mathrm{Si}(001)$ and $\mathrm{Ge}(001)$ low-miscut substrates at $400{ }^{\circ} \mathrm{C}$. Figure $3-14$ shows a monotonic decrease in order parameter with film thickness for samples grown on $\mathrm{Ge}(001)$ substrates, whereas the thickness dependence is weakly non-monotonic for films grown on $\mathrm{Si}(001)$.

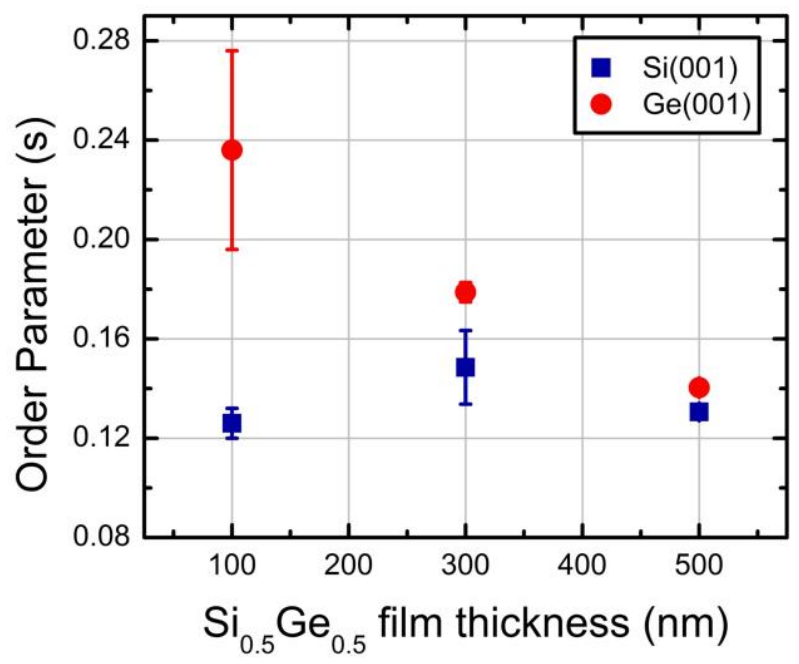

Figure 3-14: Order parameter vs thickness for samples grown on low-miscut (miscut of $\left.<0.1^{\circ}\right) \mathrm{Si}(001)$ and $\mathrm{Ge}(001)$ substrate. 


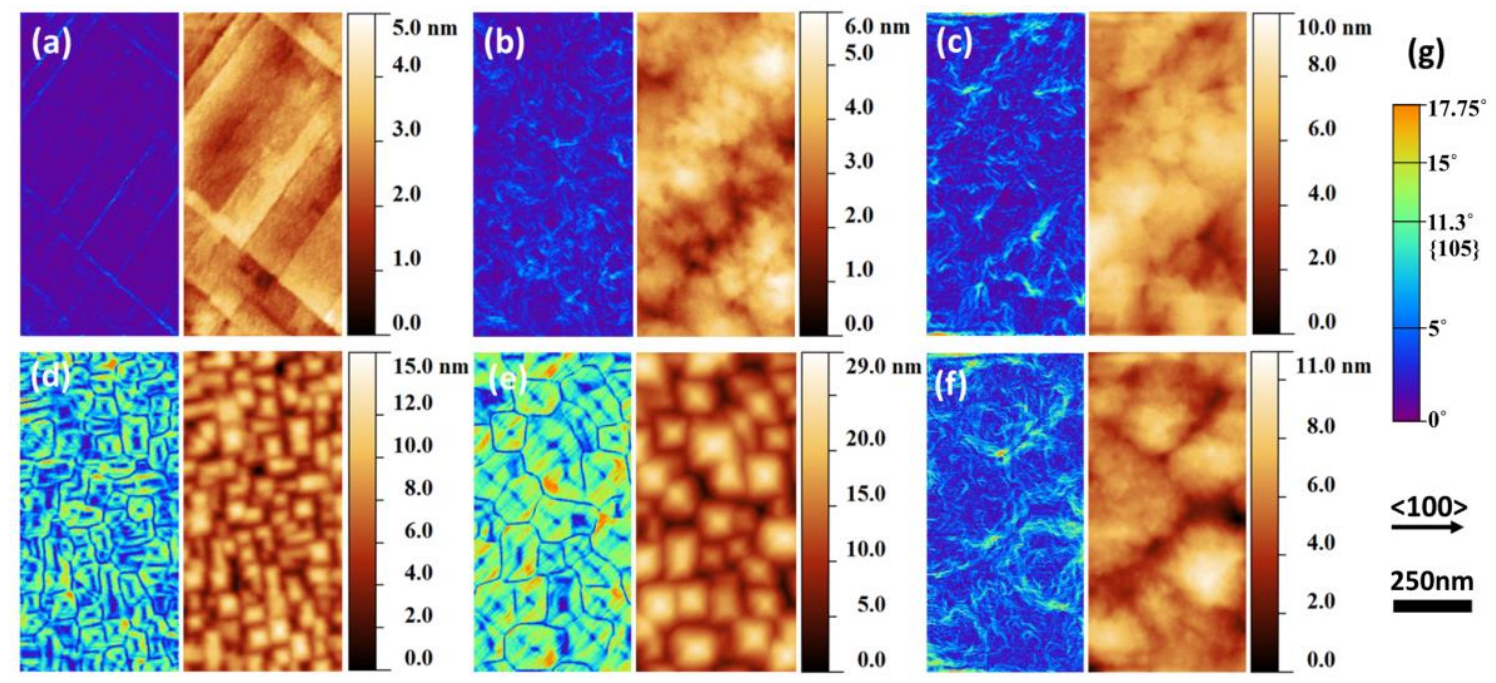

Figure 3-15: AFM micrographs and local area slope images showing the morphology of $\mathrm{Si}_{0.5} \mathrm{Ge}_{0.5}$ (a) $100 \mathrm{~nm}$, (b) $300 \mathrm{~nm}$, (c) $500 \mathrm{~nm}$ thick alloy films grown on $\mathrm{Ge}(001)$ substrate and (d) $100 \mathrm{~nm}$, (e) $300 \mathrm{~nm}$, (f) $500 \mathrm{~nm}$ thick alloys on Si(001) substrate. (g) Color bar indicates the local slope in degrees.

AFM topography scans, along with corresponding local slope maps (slope with respect to (001) surface), are shown in Figure 3-15, comparing the morphology of 100, 300 and 500 nm thick $\mathrm{Si}_{0.5} \mathrm{Ge}_{0.5}$ samples grown on low-miscut $\mathrm{Si}(001)$ and $\mathrm{Ge}(001)$ substrates. The 100 nm thick sample grown on $\mathrm{Ge}(001)$ substrates was quite smooth with extended $(001)$ terraces separated by slip traces along $\langle 110\rangle$ in-plane directions. At larger thicknesses, shallow growth mounds form, with local surface angles less than $3^{\circ}$ off $(001)$, presumably due to the presence of Ehrlich-Schwoebel barriers. ${ }^{91}$ Samples grown on Si(001) substrates showed the development of $\{105\}$-faceted, quasi-pyramidal islands that form to relieve elastic and surface energy. ${ }^{26,27}$ However, the $500 \mathrm{~nm}$ thick film on Si has smoothed, as extensive strain relaxation due to misfit dislocations removes the driving force for $\{105\}$ island formation, see Figure 3-16 (a). More details on the slope maps, and 2D slope distribution plots of the AFM images shown in Figure 3-15, are given in Appendix 3. The samples in this series were also characterized by XRD for their macroscopic strain state. 
The results show decreasing residual strain with increasing thickness for both $\mathrm{SiGe} / \mathrm{Si}$ and $\mathrm{SiGe/Ge}$ due to the introduction of misfit dislocations, see Figure 3-16 (b).
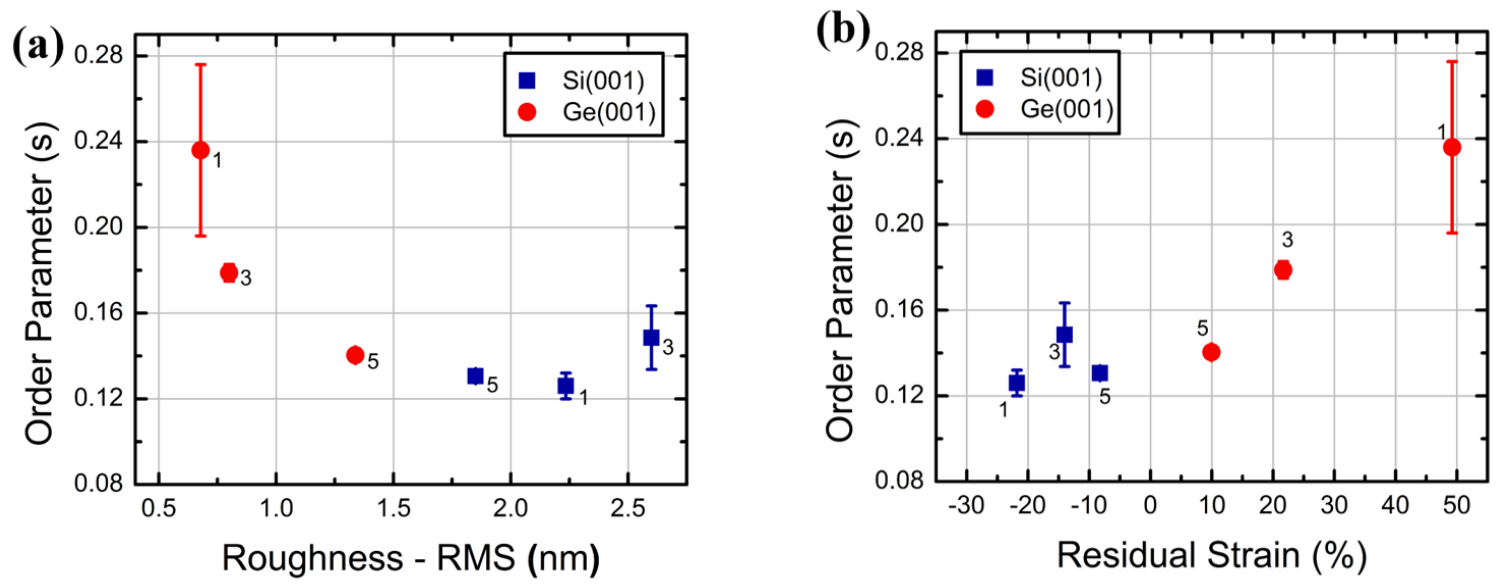

Figure 3-16: (a) Order parameter vs surface roughness, (b) order parameter vs residual strain; labels 1,3 and 5 refer to $100 \mathrm{~nm}, 300 \mathrm{~nm}$ and $500 \mathrm{~nm}$ thick alloy, respectively. A residual strain of $50 \%$ means that half the initial mismatch strain has been relaxed.

\subsubsection{Comparative MBE grown samples}

We performed a comparative study with $300 \mathrm{~nm}$ thick $\mathrm{Si}_{0.5} \mathrm{Ge}_{0.5} / \mathrm{Si}(001)$ alloys grown by a conventional Riber MBE (IM2NP-France, courtesy of Dr. Isabel Berbezier) at growth temperatures: $400,450,500$ and $550{ }^{\circ} \mathrm{C}$. Figure 3-17 shows a plot comparing the order parameter between the two series of sample grown with a different MBE setup. Similar to the temperature varied samples grown on low-miscut $\operatorname{Si}(001)$ substrate, see Section 3.4.1, these samples also exhibited a highly symmetric distribution of intensity amongst all the four orientation domains. Similar to the samples grown in the UVa MBE, for Riber MBE a growth temperature of $400{ }^{\circ} \mathrm{C}$ exhibited maximum $\mathrm{S}$, where the overall ordering sharply decayed at higher growth temperatures, with $S_{\max }=0.13$. 


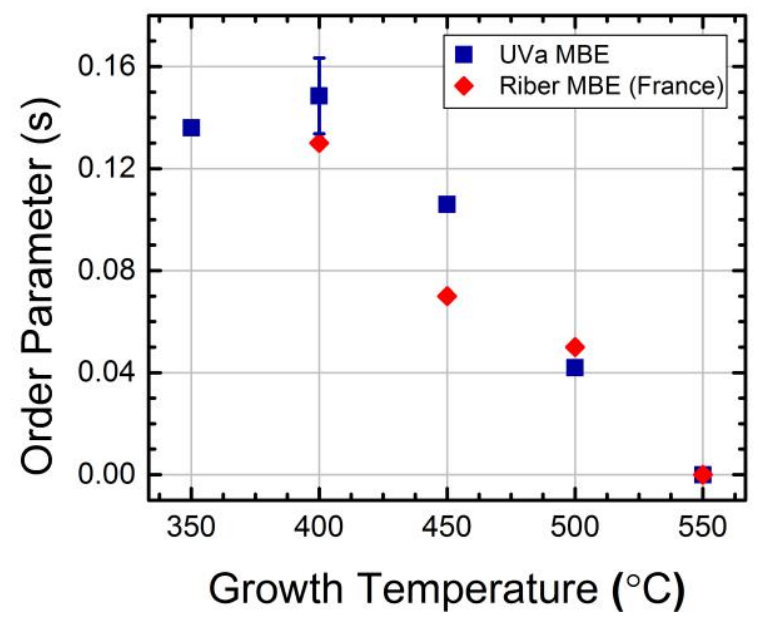

Figure 3-17: Order parameter for $300 \mathrm{~nm}$ thick $\mathrm{SiGe} / \mathrm{Si}$ samples grown at various temperatures with two different MBE setups.

\subsection{Discussion}

We have explored the propensity towards metastable chemical ordering in $\mathrm{Si}_{0.5} \mathrm{Ge}_{0.5}$ alloys during MBE growth, over a broad range of conditions. In all cases examined here, the order parameter we obtained has never exceeded $S=0.24$, hence ordering is limited. For the most part, these order parameters are consistent with the literature, although it must be noted that while many studies have shown evidence of ordering by the presence of superlattice reflections, few have accurately and correctly quantified S. Significantly higher order parameters were reported for very thin SiGe alloy films (up to $\mathrm{S}=0.71$ at 5 $\mathrm{nm}$ thickness) based on in situ x-ray surface diffraction measurements. ${ }^{61}$ An order parameter of 0.64 was claimed in a standard XRD analysis of $500 \mathrm{~nm}$ thick $\mathrm{Si}_{0.5} \mathrm{Ge}_{0.5}$ films grown at $390{ }^{\circ} \mathrm{C}$, but details on the calculation were not provided. ${ }^{79}$ A thorough characterization of order parameter was performed by Tischler, et al. ${ }^{74}$, who obtained $\mathrm{S}=$ 0.28 for a $300 \mathrm{~nm}$ thick $\mathrm{Si}_{0.5} \mathrm{Ge}_{0.5}$ film grown on $\mathrm{Si}(001)$ at $400{ }^{\circ} \mathrm{C}$ by $\mathrm{MBE}$. It does not appear Tischler, et al., considered the volume-fraction effect of multiple domains as 
embodied in Equation (3-3) here, hence we have doubled their reported value of $S=0.14$. Our film grown under nominally identical conditions to theirs only exhibits $S=0.16$. Our MBE uses UHV magnetron sputtering, so it is possible that a small fraction of energetic species could create surface damage that affects diffusion-related mechanisms, or the surface reconstruction itself, thereby affecting ordering kinetics. However, we did compare with $\mathrm{Si}_{0.5} \mathrm{Ge}_{0.5} / \mathrm{Si}(001)$ alloys grown by a conventional Riber MBE via evaporation, under otherwise identical conditions, and found that material had similar, or slightly lower, values of $\mathrm{S}$, see Section 3.4.5. And, since we are able to obtain higher order parameters on $\mathrm{Ge}$ substrates, bombardment effects cannot be entirely limiting.

There are three key observations from our results that are non-trivial to reconcile against one another:

1. The highest order parameters were obtained for SiGe growths on $\mathrm{Ge}(001)$, especially in the thinnest film, which is extremely smooth, and then ordering rapidly decays as surface roughening, and thus surface step density, simultaneously increases with film thickness. Is ordering reduced by high step density?

2. For $\mathrm{SiGe}$ growths on low-miscut $\mathrm{Si}(001)$, the order parameter is only half the maximum value observed for growth on Ge. Furthermore, ordering persists, and the order parameter is roughly constant as a function of film thickness, despite a relatively extreme evolution of the surface morphology to form $\{105\}$-faceted islands, with little exposed (001) terrace, followed by development of much shallower mounds as the strain relaxes. Is ordering insensitive to high step density?

3. For $\mathrm{SiGe}$ growth on high-miscut $\mathrm{Si}(001)$, the RMS order parameter is the same as for low-miscut $\mathrm{Si}$, but the distribution of ordering amongst the four spatial ordering 
variants is clearly modified in a way consistent with changes in density of specific steps with miscut angle and direction (more on this below). Is ordering enhanced by high

\section{step density?}

Steps, particularly double-height $\mathrm{D}_{\mathrm{B}}$ steps, are thought to play a significant role in establishing long-range, uniaxial ordering in SiGe. Clearly, our results provide a contradictory picture on the role of steps. For SiGe films grown on Ge substrates, it is clear that order parameter is the highest in thinner SiGe films, where growth takes place on large, extremely smooth (001) terraces, having RMS roughness of only $0.13 \mathrm{~nm}$ for the $100 \mathrm{~nm}$ thick film. This clearly indicates that large step densities are not required a priori, and it seems unlikely that double-height steps are present to a significant degree. The lack of $D_{B}$ steps, while still obtaining significant order, are qualitatively consistent with findings by Cahill. $^{61}$

As the film surface roughens with increased film thickness during growth on Ge (001), the order parameter goes down as the step density increases. However, we suggest that this is not due to the roughening per se, but is related to shearing-induced disorder with progressive strain relaxation. It is straightforward to show that four of the eight active $1 / 2<110>/\{111\}$ slip systems will shear any particular ordered domain to produce an antiphase boundary (APB). For a film that is $50 \%$ relaxed, the mean dislocation spacing is D $=b_{\mathrm{e}} / 0.01=19 \mathrm{~nm}$, where $b_{\mathrm{e}}$ is the in-plane edge component of the burgers vector for $\mathrm{a} 60^{\circ}$ misfit dislocation. For $90 \%$ relaxation, the spacing decreases to $11 \mathrm{~nm}$. This increase in APB density should correlate with the misfit dislocation density, and this will broaden the superlattice reflection in $\mathrm{x}$-ray diffraction. Using an expression from Lutterotti and Gialanella $^{92}$, the angular broadening of the $1 / 2(111)$ reflection associated with APBs is 
estimated to be $0.95^{\circ}$ for $11 \mathrm{~nm}$ APB spacing. They quantified how the $\mathrm{x}$-ray order parameter decreased with increasing deformation in ordered $\mathrm{Ni}_{3} \mathrm{Al}$ alloys, and the degree of disordering is consistent with our observations here. Growth of the same alloys on $\mathrm{Si}(001)$ produces less ordering than on Ge substrates. Growth on $\mathrm{Si}$ also lead to more facile strain relaxation - Figure 3-16 (b) shows that at only $100 \mathrm{~nm}$, the alloy is already $79 \%$ relaxed, vs. $50 \%$ relaxation for $100 \mathrm{~nm} \mathrm{SiGe} \mathrm{on} \mathrm{Ge.} \mathrm{This} \mathrm{is} \mathrm{consistent} \mathrm{with} \mathrm{shearing-}$ induced disorder.

The key role of steps in promoting ordering is strongly suggested by the results for highmiscut samples shown in Figure 3-13. The particular ordering variants that are suppressed by the miscuts towards [100] and [110] are consistent with geometrically-necessary changes in the relative step population. It is useful to compare the average degree of order across the variants for growth on low- and high-miscut Si substrates. Since the volume fractions are unknown a priori, however, we cannot compute the volume-weighted average. This can be avoided by using the root-mean-square average, $S_{\mathrm{RMS}}=\left\langle\mathrm{S}^{2}\right\rangle^{1 / 2}$, where, using Equation 3-3, we get

$$
\left\langle S^{2}\right\rangle=\sum_{i} S_{i}^{2} v_{i}=\left(\frac{1}{Q}\right) \sum_{i} \frac{I_{h^{\prime} k^{\prime} I^{\prime}}^{i}}{I_{h k l}}
$$

We find that $\mathrm{S}_{\mathrm{RMS}}$ is essentially identical for the low- and high-miscut samples. This can be interpreted as the changes in step density associated with deliberate miscut do not change the actual degree of order $\left(\mathrm{S}_{\mathrm{i}}\right)$, but they do bias the relative volume fractions of the ordered variant domains. Nonetheless, changes in the relative volume fractions during the growth on miscut wafers shows that step edges do influence the ordering process. 
We also verified that changes in ordering on the different $\langle 111\rangle$ variants is not produced by changes in dislocation activity as a result of symmetry-breaking, induced by the miscut, of the relevant slip systems. For all eight active slip systems, we calculated the resolved shear stress on each by rotating the biaxial stress tensor, defined in the reference axes of the miscut substrate, onto a new reference frame defined by the burgers vector + slip plane normal. ${ }^{93}$ We find that there are offsetting increases and decreases of the resolved shear stress, of order $\pm 5 \%$ of the value for a non-miscut system. First, the effect is clearly small, and second, the offsetting changes suggest that there will not be an enhanced APB density on any specific ordered variant.

The results for growth on low-miscut Si may be the hardest to understand. As the film thickens, the surface evolves from a planar wetting layer, through $\{105\}$-faceted pyramidal islands with very little exposed (001), and then to irregular mounds as misfit dislocations relax the elastic energy responsible for the faceted islands. Throughout this process, ordering persists, and the order parameter is essentially unchanged. The $\{105\}$ facet in its fully-rebonded, minimum energy state ${ }^{94}$, cannot be thought of as being narrow sections of (001) terrace bound by a high step density. Hence it is not clear why $\mathrm{L}_{1}$-like ordering would occur on a true $\{105\}$ facet. However, we observe nominal $\{105\}$ facets in films with relatively low strains due both to the alloy composition (vis-à-vis pure Ge islands where the facets are best studies) and due to the extensive strain relaxation from misfit dislocations. Under these conditions, and at these relatively low growth temperatures, it is quite possible that the $\{105\}$ island faces are not fully rebonded, therefore retaining some aspects of the $2 \times 1$ reconstructed surface structure that continues to facilitate ordering via the mechanisms discussed above. An alternative possibility is that the atomic relaxations 
underneath the $\{105\}$ surface are similar to those under the (001)-2x1, promoting similar atomic incorporation biases as discussed by Tersoff, et al. ${ }^{72,78}$ To the best of our knowledge, this has not been examined in the literature.

\subsection{Summary}

We have performed a parametric study for the heteroepitaxial growth of $\mathrm{Si}_{0.5} \mathrm{Ge}_{0.5}$ alloy by MBE on $\mathrm{Si}(001)$ and $\mathrm{Ge}(001)$ substrates, to gain better insight into the growth factors affecting the order parameter of these alloys. Key results include:

- The effect of equivalent spatial ordering variants on the calculated order parameter was explicitly considered here. Our expression for the diffracted intensity in a superlattice reflection explicitly accounts for the volume fractions of each variant. This can have a large effect on the reported value of S.

- The observed order parameter never exceeded $S=0.24$ across a range of growth temperatures, film thicknesses, substrate miscuts, and substrate types ( $\mathrm{Si}$ vs. Ge). Claims in the literature of much higher order parameters could not be reproduced here, despite a survey over multiple growth parameters suggested to be important to ordering in the literature, and this was true in a conventional MBE as well as in our sputtering-based MBE. We feel this may be due to inaccurate calculations of the order parameter in the relatively few works where quantification was performed.

- Smoother films, with extensive (001) terrace, led to the highest degree of order, and the order parameter subsequently decayed as surface step density increased with increasing film thickness.

- The sign and magnitude of the strain was not directly important to ordering, other than for helping determine the evolution of the surface roughness and strain relaxation. Strain 
relaxation is believed to reduce the order parameter when the degree of relaxation is large, by shearing of ordered variants by misfit dislocations to create APBs.

- Ordering persists, albeit at low order parameters, during extensive roughening and even nominal $\{105\}$ faceting. Persistent $\mathrm{L}_{1}$ ordering is hard to understand based on models suggesting that the $2 \times 1$ reconstructed $\mathrm{Si}(001)$ surface is crucial to the development of order. One possible explanation is that the $\{105\}$ facets are not fully rebounded at these growth conditions, which would lead to local regions of step configurations similar to those on the $2 \times 1$ surface.

- The experimental results presented here are not able to entirely distinguish the dominant ordering mechanism between the proposed theoretical models. However, growth on deliberately miscut substrates did not change the average order parameter, but did change the relative volume fraction of the ordered spatial variants. This indicates that step configurations on the growth surface do play a role in long-range order.

- Finally, previous calculations on how ordering affects thermal transport suggest that much higher order parameters are required in order to obtain a significant effect. ${ }^{16}$ 


\section{Chapter 4: Directed self-assembly of $\mathrm{Si}_{1-\mathrm{x}} \mathrm{Ge}_{\mathrm{x}}$ QDs}

As discussed in Chapter 1, a quantum dot mesocrystal (QDMC) is a highly ordered 3D array of QDs epitaxially embedded in a matrix material with a potential to tailor the electronic structure and ultimately generate useful new properties. For the successful growth of such a meso-scale structure, the initial underlying 2D template or the substrate pattern morphology plays a critical role. Growth of QDs on templated surfaces allows us to achieve not only spatial ordering but also morphological ordering. Various lithographic techniques are required to create such $2 \mathrm{D}$ templates on the growth surface to predefine nucleation sites to yield high degree of QD uniformity and ordering essential for a QDMC.

In this dissertation, we present a detailed investigation of the QD evolution on patterned $\mathrm{Si}$ (001) substrates as a function of various growth parameters and the pattern-related variables. Multilayer growth to form QDMCs is not covered here however, the work serves as a core foundation to the ongoing efforts in our group for the QDMC growth along with its electronic and thermal transport measurements. In this chapter, we will review the key literature and discuss the science of directed self-assembly of heteroepitaxial QDs.

\subsection{Introduction}

Coherently strained $\mathrm{Si}_{1-\mathrm{x}} \mathrm{Ge}_{\mathrm{x}}$ QDs that self-assemble during epitaxial growth on $\mathrm{Si}(001)$ exhibit a broad size distribution and spatial disorder. ${ }^{26,27}$ Figure 4-1 shows the AFM image of such randomly self-assembled QD structures grown on a non-patterned $\mathrm{Si}(001)$ substrate. For potential device applications, control over absolute size as well as position of these dots are required. The basic approach to homogenize the size and position of QDs can be realized through subsequent multilayer growth. The concept is schematically illustrated in Figure 4-2. $\mathrm{Si}_{1-\mathrm{x}} \mathrm{Ge}_{\mathrm{x}}$ QDs self-assemble on $\mathrm{Si}$ (001) via the S-K growth mode 
(a)

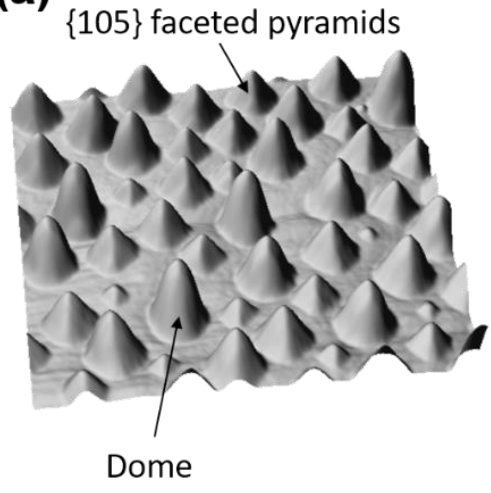

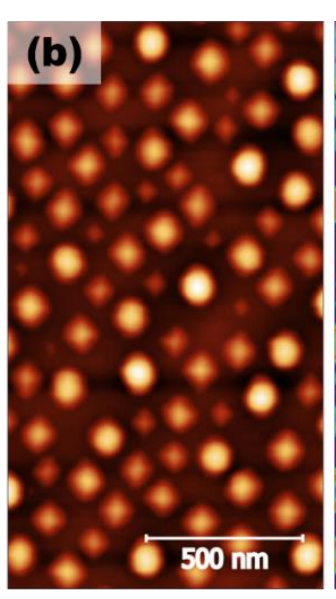

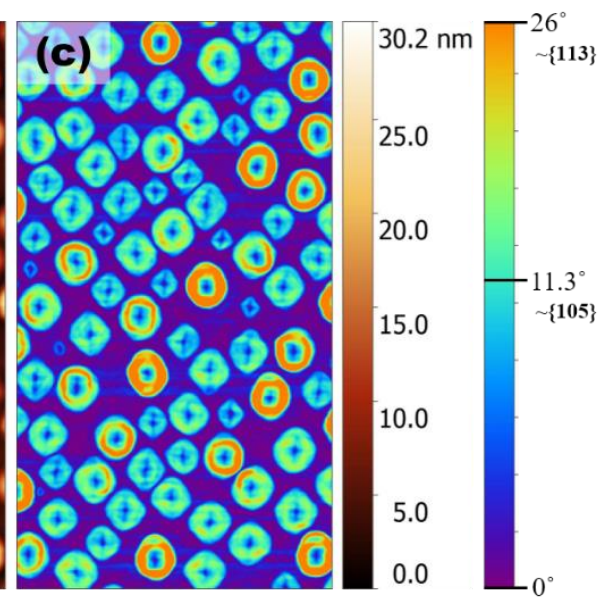

Figure 4-1: AFM image showing $\mathrm{Si}_{0.5} \mathrm{Ge}_{0.5}$ QDs nucleating randomly on a $\mathrm{Si}(001)$ substrate: (a) 3D perspective view, (b) topography image, and (c) local area slope image outlining the facets of quasi-pyramidal and dome morphology.

of WL followed by formation of coherently-strained islands. If a Si layer is overgrown on the QDs, and another layer of $\mathrm{Si}_{1-\mathrm{x}} \mathrm{Ge}_{\mathrm{x}}$ is then deposited, the 2nd layer of dots will tend to align above the first layer due to the inhomogeneous strain distribution present on the surface of a sufficiently thin Si interlayer. ${ }^{95}$ Repetition of this process leads to verticallyaligned, but laterally random, QD structures called quantum dot superlattices (QDSL's), see Figure 4-2(c). However, if the QDs could be periodically arranged in the very first layer by creating surface templates, the first layer of laterally ordered QDs can then be used as 2D seed "crystals" that can be propagated into the third dimension through subsequent multilayer growth. Such a highly ordered array of QDs in 3D is called a quantum dot mesocrystal (QDMC), see Figure 4-2(d).

The only reported 3D QDMC arising from intrinsic self-assembly is for the $\mathrm{PbSe} / \mathrm{PbEuTe}$ superlattice material system, where elastic anisotropies drive the $3 \mathrm{D}$ alignment of $\mathrm{PbSe}$ quantum dots into mesocrystals. ${ }^{96}$ For the Group IV and III-V systems, directed selfassembly is required to produce QDMCs. The very first demonstration of lateral ordering 

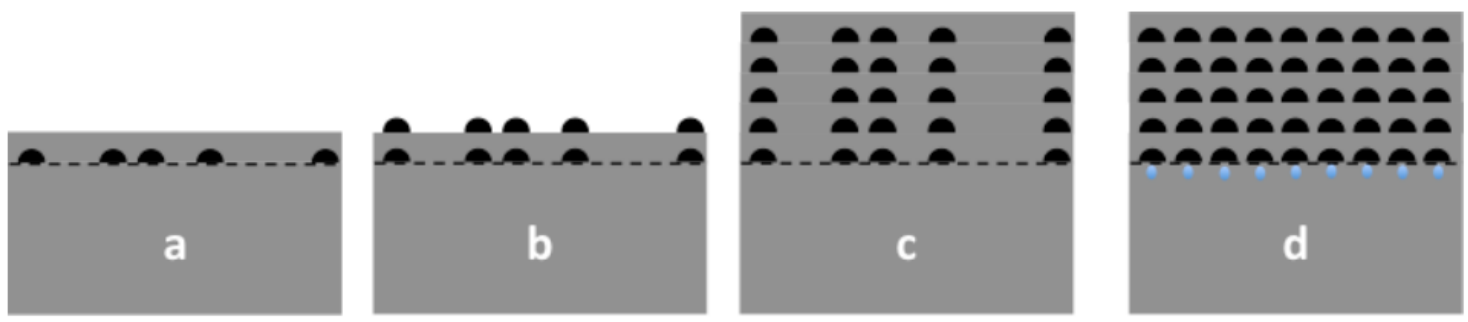

Figure 4-2: (a) Single layer of S-K Ge QDs, capped with Si, but randomly oriented inplane. (b) A second layer aligns to the first via propagated strain fields on the Si cap surface. (c) Repeating the Ge/Si growth sequence leads to a QDSL. (d) If the first layer of Ge QDs are made to be 2D periodic, then subsequent Ge/Si layering leads to a QDMC

in $\mathrm{SiGe}$ system was performed without any lithographic techniques where miscut $\mathrm{Si}(001)$ substrates were used to direct the self-assembly of QDs on the step edges. ${ }^{97,98}$ However, lithographic techniques are required to create templates on the growth surface to predefine nucleation sites to yield a high degree of QD uniformity and ordering.

As described by Shchukin et al., ${ }^{99}$ there are five classes of ordered nanopatterns or nanostructures where the periodic structures influence the surface chemical potential to drive the QD growth/ordering process, see Figure 4-3. Our approach in this work best fits with obtaining the ordered nanostructures as shown in Figure 4-3(c) and eventually a QDMC as shown in Figure 4-3(e).
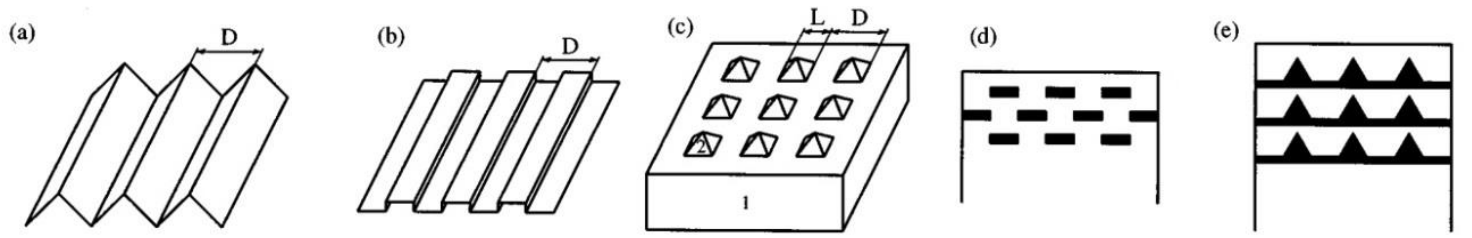

Figure 4-3: Various classes of ordered nanostructures: (a) periodically faceted surfaces; (b) surface structures of planar domains; (c) ordered array of coherently strained islands; (d) cross-sectional scheme of a multisheet array of 2D islands; (e) cross-sectional scheme of a multisheet array of 3D islands (QDMC). ${ }^{99}$ 
In order to modify the growth substrate to create a $2 \mathrm{D}$ template for directing the selfassembly process of QDs, various lithographic techniques like electron-beam lithography $(E B L)^{100}$, ion-beam lithography ${ }^{101-103}$ and extreme ultraviolet interference lithography $\left(\right.$ EUV-IL) ${ }^{24,104}$ can be used to create variety of 2D surface templates like pits ${ }^{104-106}$, stripe arrays $^{107-109}$, buried stressor (strain field modulation of the growth surface) ${ }^{110}$ and oxide windows ${ }^{111}$, see Figure 4-4.

As shown in Figure 4-4, creating 2D templates via lithographic approach is an effective method to direct the self-assembly of QDs where these QDs are localized at the edges or in the patterns. However, fundamental understanding of the effects of surface modification on the QD evolution is a necessity in order to successfully produce patterns with higher dot
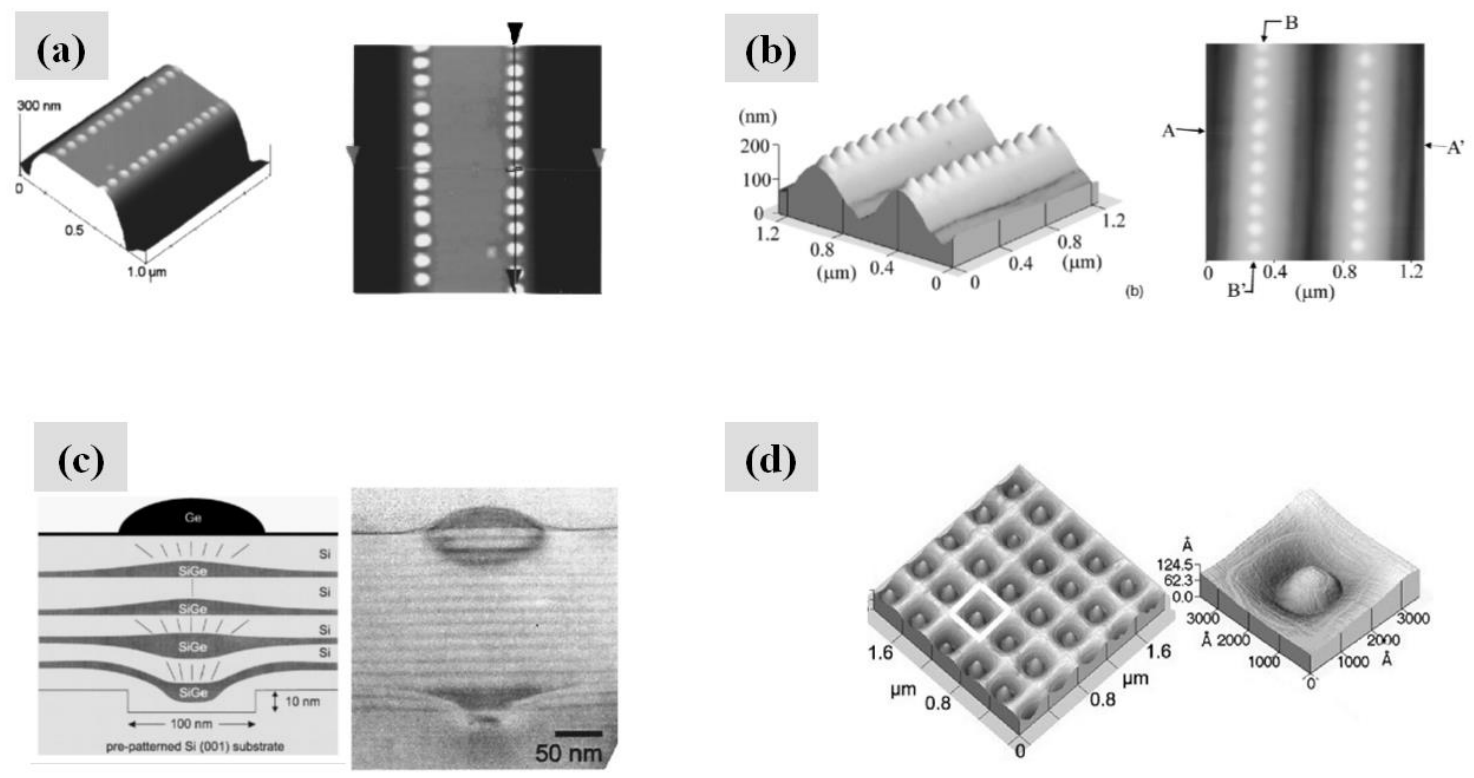

(d)

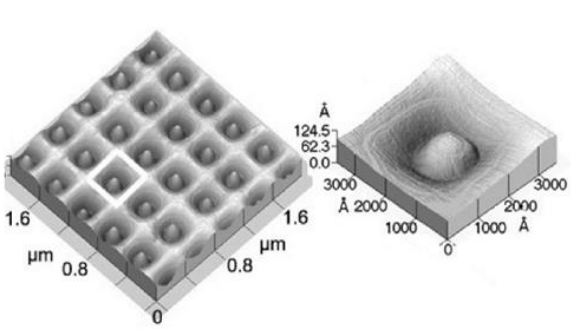

Figure 4-4: (a) QDs ordering along <100> near the edges of a lithographically defined $\mathrm{Si}(001)$ plateau ${ }^{107}$, (b) QDs aligned along a $<110>$ oriented ridges of the Si strip mesas ${ }^{108}$, (c) trench formation on the substrate resulting in the strain field modulation to direct QD formation on a flat top surface ${ }^{110}$, (d) QDs nucleating inside the pits of a pit-patterned substrate yielding highly ordered dots ${ }^{143}$. 
density and tunable inter-dot spacing. In the next section, we will discuss the science of QD localization and formation on patterned substrates.

\subsection{Surface chemical potential: 2D template}

The evolution of SiGe QDs on a flat $\mathrm{Si}(001)$ surface was described in Chapter 1, where the strain induced QDs are formed via surface diffusion of adatoms. When a flat $\mathrm{Si}(001)$ surface is modified to form a pit, the chemical potential of the surface will also be modified. Therefore, a proper understanding of the surface thermodynamics which rules the surface diffusion on a modified surface is needed. For a modified surface, the surface free energy is influenced where the chemical potential can be described by ${ }^{33,112}$

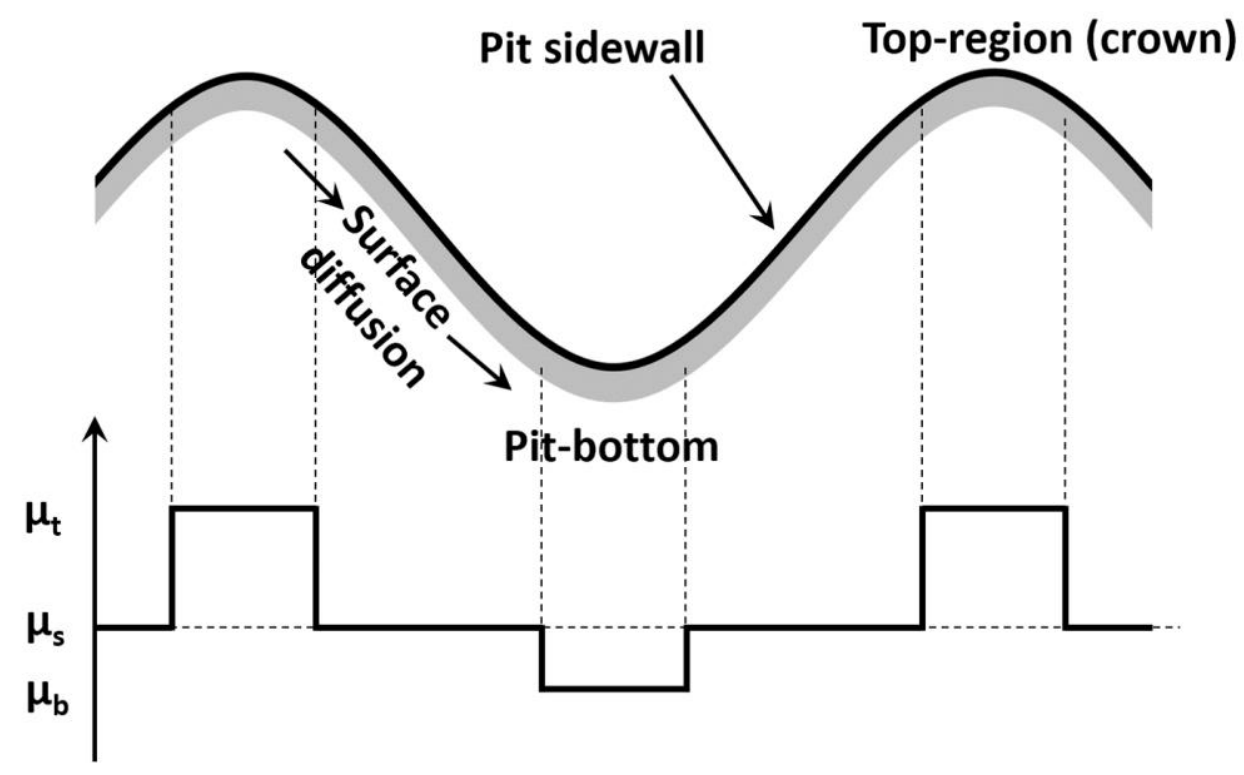

Figure 4-5: Schematic of a pit profile with top (t), bottom (b) and sidewall (s). Due to the curvature, the chemical potential is minima at the pit-bottom $\left(\mu_{t}>\mu_{s}>\mu_{b}\right){ }^{144}$ 


$$
\mu(r)=\mu_{0}+\Omega \gamma \mathrm{k}(\mathrm{r})+\Omega \mathrm{E}_{e l}(r),
$$

where $\mu_{0}$ is the chemical potential of the planar surface, $\gamma$ is the orientation dependent surface energy, $\Omega$ is the atomic volume, $\mathrm{E}_{\mathrm{el}}(\mathrm{r})$ is the elastic energy density computed on the film free surface and $k(r)$ is the local curvature at the position $r$ on the surface. The second term in Equation 4-1 describes the energetic cost of an increase in the surface area due to local surface curvature and the third term describes the effect of strain relaxation associated with surface curvatures. For a patterned surface with a profile as shown in Figure 4-5, the capillarity forces will always try to flatten/ smoothen the surface by driving the mass transfer towards the pit bottom. However, during the QD self-assembly process if capillary forces were the only driving force the patterned substrate would result in flattening which is not the case as shown in Figure 4-4. QDs are strain induced structures, thus strain relaxation must play an important role during their evolution. The third term in Equation 4-1 incorporates the effect of strain relaxation. And the strain relaxation favors the mass diffusion towards concave region (top of the ridge) as compressed SiGe layer can stretch out more easily on a concave region. Therefore, the balance between surface energy and strain relaxation along with the pattern geometry plays a key role during the growth and localization of QDs. Numerous theoretical and experimental works have been published to understand the growth science. 


\subsection{Growth on patterned substrates}

Lithographic processes have been very successful in directing the self-assembly of QDs to produce highly ordered arrays. Grydlik, et al., ${ }^{105}$ outlined important parameters influencing the growth of ordered QDs on a pit-patterned $\mathrm{Si}(001)$ substrates including: pit morphology, deposition rate and volume, growth temperature, pattern period, buffer layer growth and film composition. The pattern pitch for their experimental study ranged from $400-3400$ nm where high quality arrays of dots nucleated within the pits, see Figure 4-6. The initial
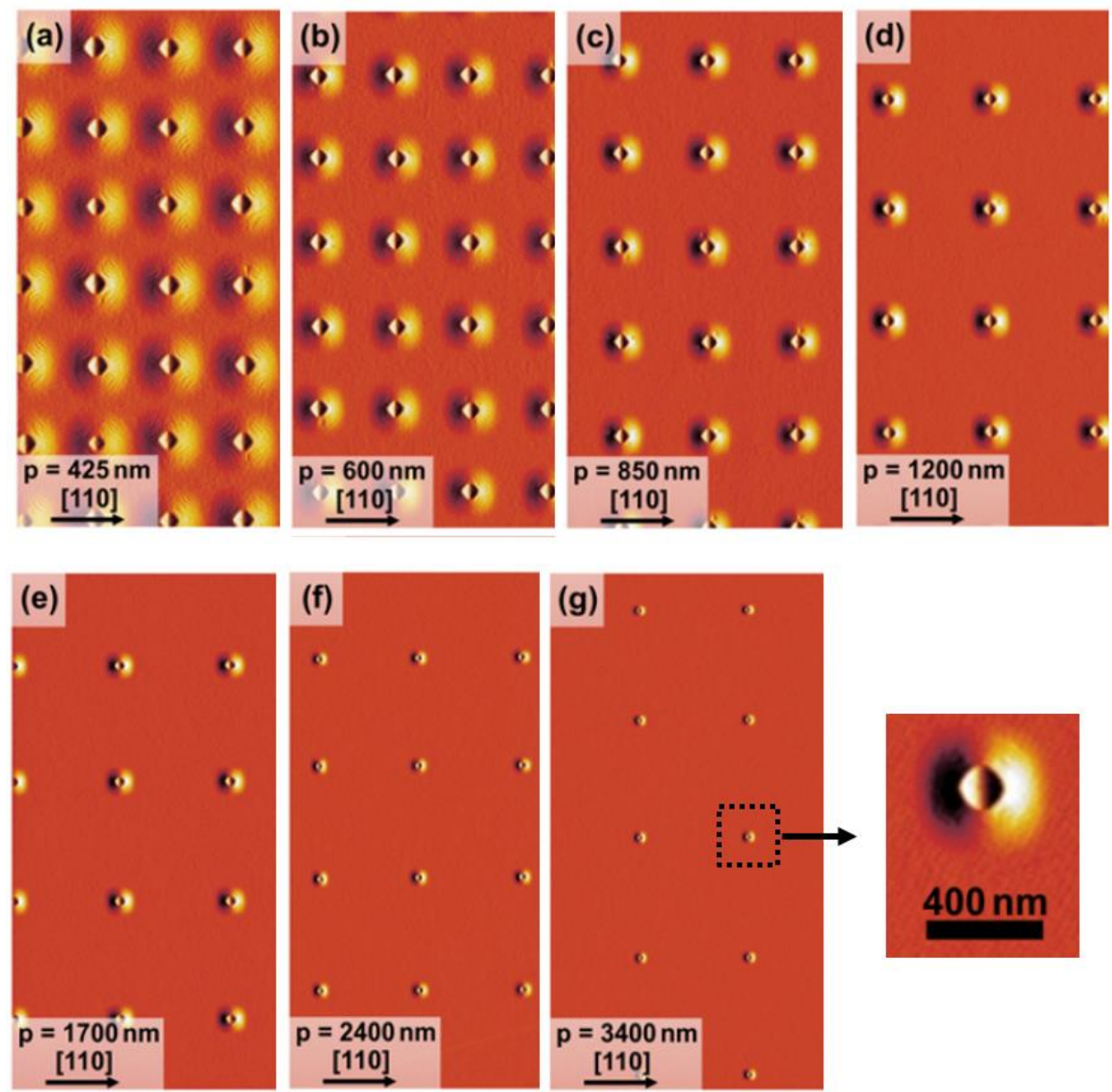

Figure 4-6: AFM images in derivative mode showing the results for 3 ML of Ge deposited on a pit-patterned $\mathrm{Si}(001)$ substrate at $700{ }^{\circ} \mathrm{C}$ with various pitch $\left(\lambda_{\mathrm{p}}\right)$ ranging $425 \mathrm{~nm}-$ $3400 \mathrm{~nm} .^{105}$ 
pit shape and size along with the combination of Si buffer layer growth conditions were found to be critical in defining the QD nucleation sites.

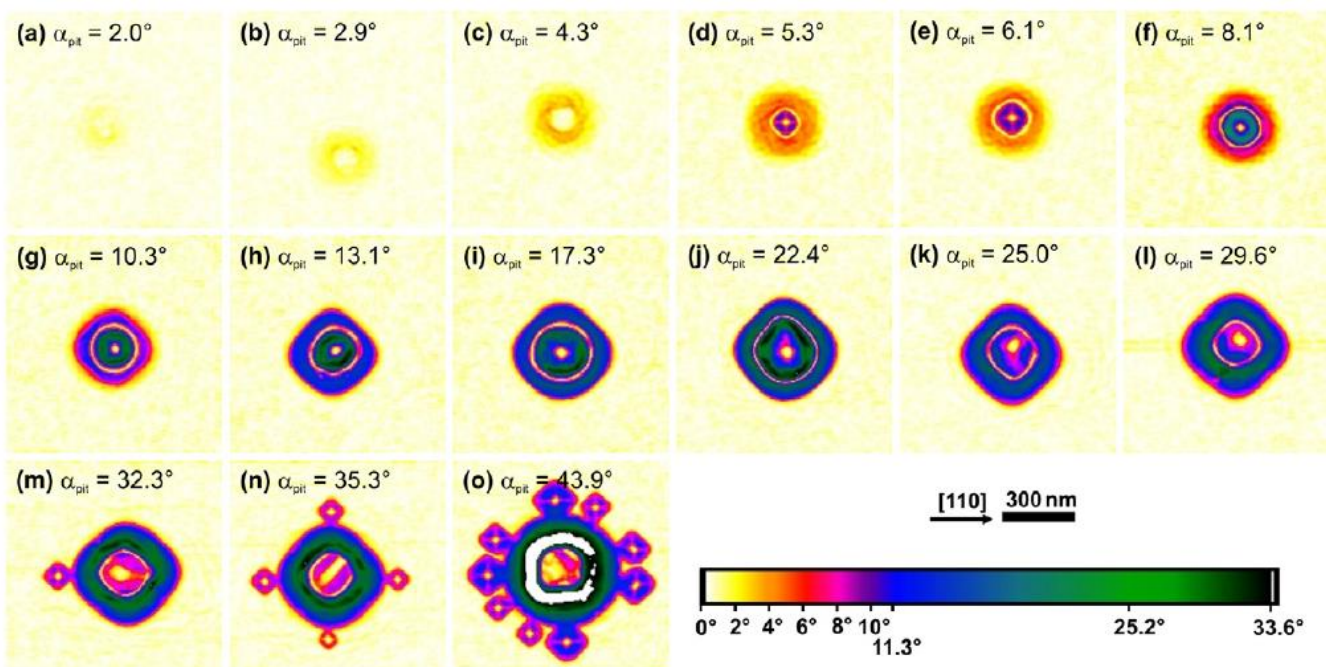

Figure 4-7: Local area slope images for a single pit comparing the position/ localization of QDs relative to the pit-sidewall angle $\left(\alpha_{\text {pit }}\right)$. QDs were observed to localize at the edges for $\alpha_{\text {pit }}>30^{\circ} .105$

Grydlik, et al., in their work also found Ge QDs nucleating inside shallow pits (pit-sidewall inclination angle $<30^{\circ}$ ) but for pits with higher inclination angle, dots were observed to localize at the edges, see Figure 4-7. Vastola, et al., ${ }^{113,114}$ used elastic theory and finite
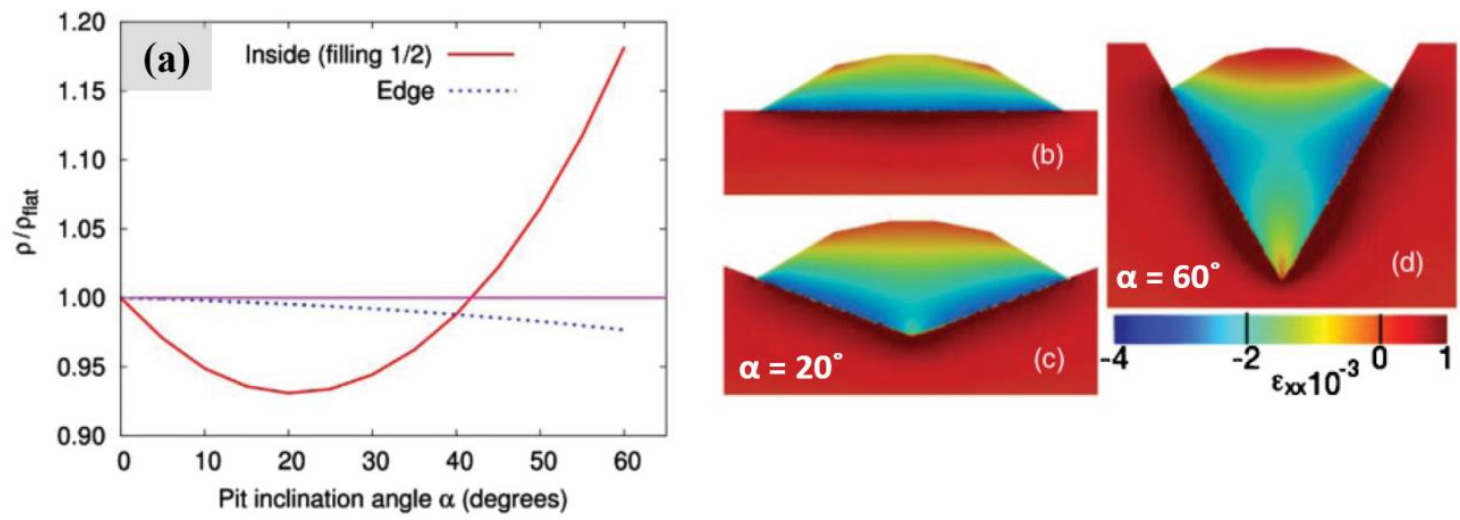

Figure 4-8: (a) Elastic energy density ( $\rho$ ) vs pit inclination angle $(\alpha)$ plot, $\rho$ is normalized to the energy density of Ge dome on a flat surface. Elastic energy maps for domes with (a) $\alpha=0^{\circ}$ (flat surface), (b) $\alpha=20^{\circ}$, and (c) $\alpha=60^{\circ} .113$ 
element analysis (FEA) model to correlate the strain relaxation of QDs and pit-sidewall inclination angle. Their theoretical results show that surface-minimization and elastic relaxation are the driving force for dots to nucleate inside the shallow pits such that the dots are more strain relaxed inside the shallow pit compared to growth on a flat (001) surface, see Figure 4-8. Schulli, et al., ${ }^{115}$ verified this experimentally using precision x-ray diffraction showing that dots inside the pits are more strain relaxed compared to dots on a flat (001) surface.
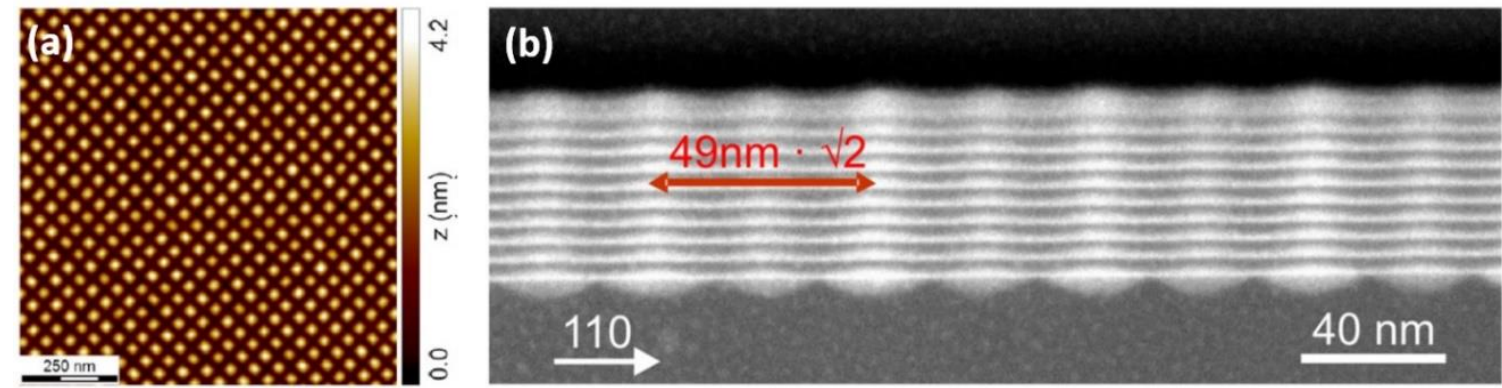

Figure 4-9: (a) $1 \times 1 \mu \mathrm{m}^{2}$ AFM image of the top layer of QDMC with lateral period of 49 $\mathrm{nm}$. (b) Cross-section STEM image of the sample shown in (a). ${ }^{116}$

For QD growth on pattern periodicities ranging from 35 - 100 nm, Dais, et al., ${ }^{104,116,117}$ have reported the QDs to nucleate in the patterned pits, see Figure 4-9. For their growth, the substrates were patterned with extreme ultraviolet interference lithography (EUV-IL) yielding $49 \times 49 \mathrm{~nm}, 42 \times 42 \mathrm{~nm}$ and $35 \times 35 \mathrm{~nm}$ pit patterns over an area of $25 \times 25 \mu \mathrm{m}^{2}$. For their growth, a $10 \mathrm{~nm}$ thick $\mathrm{Si}$ buffer layer was deposited at $350{ }^{\circ} \mathrm{C}$ followed by a $5 \mathrm{ML} \mathrm{Ge}$ at $540{ }^{\circ} \mathrm{C}$ which resulted in the nucleation of dots inside the pits. The cross-sectional scanning transmission electron microscopy (STEM) image for multilayer growth, with alternate stacking of $5 \mathrm{~nm}$ of Si spacer layers and $4 \mathrm{ML}$ Ge dots at $560{ }^{\circ} \mathrm{C}$, clearly shows that QDs are forming inside the pits, see Figure 4-9(b). 
Recently, our group (C. Duska) has successfully demonstrated patterned growth of QDs with lateral periodicities down to $50 \mathrm{~nm}$, see Figure $4-10 .{ }^{102}$ The Si surface was patterned using focused ion beam (FIB) and defect selective etching, resulting in a continuously height-modulated, "egg-carton" growth surface morphology. After the growth of QD arrays at $450{ }^{\circ} \mathrm{C}$, the underlying surface template was examined by etching the Ge QD layer in warm water, which does not etch the $\mathrm{Si}$. The direct 1-to-1 comparison of quantum dot and their growth sites indicated that Ge QDs preferentially nucleate in the crown regions unlike pit-nucleation for the similar pattern periodicity as observed by Dias, et al., see Figure 4-10. Similar observation of island nucleating in crown regions of pit-patterns has also been reported by other groups where the crown nucleation was observed to depend strongly on the growth temperature, see Figure 4-11. ${ }^{101}$
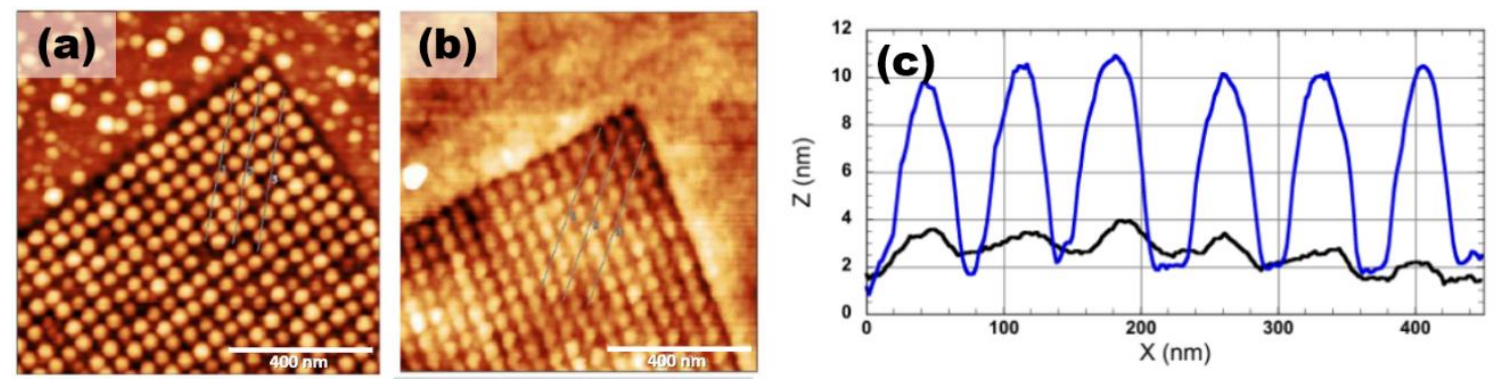

Figure 4-10: AFM data for $50 \mathrm{~nm}$ pitch pattern showing (a) Ge QDs and (b) same region of Si surface after Ge was etched off. (c) AFM linescans from the Ge QDs (blue) and $\mathrm{Si}$ surface (black) after etching Ge QDs.

As QDs grow via the S-K growth mode, the wetting layer should definitely play a key role in directing the nucleation sites. Zhang, et al., ${ }^{118}$ using selective etching experiments showed that islands form inside the pits before the WL reaches the critical thickness on the planar parts in between the pits. At finer lengthscales, when adjacent pit edges approach each other, the growth surface can no longer be viewed as discrete pits surrounded by flat 

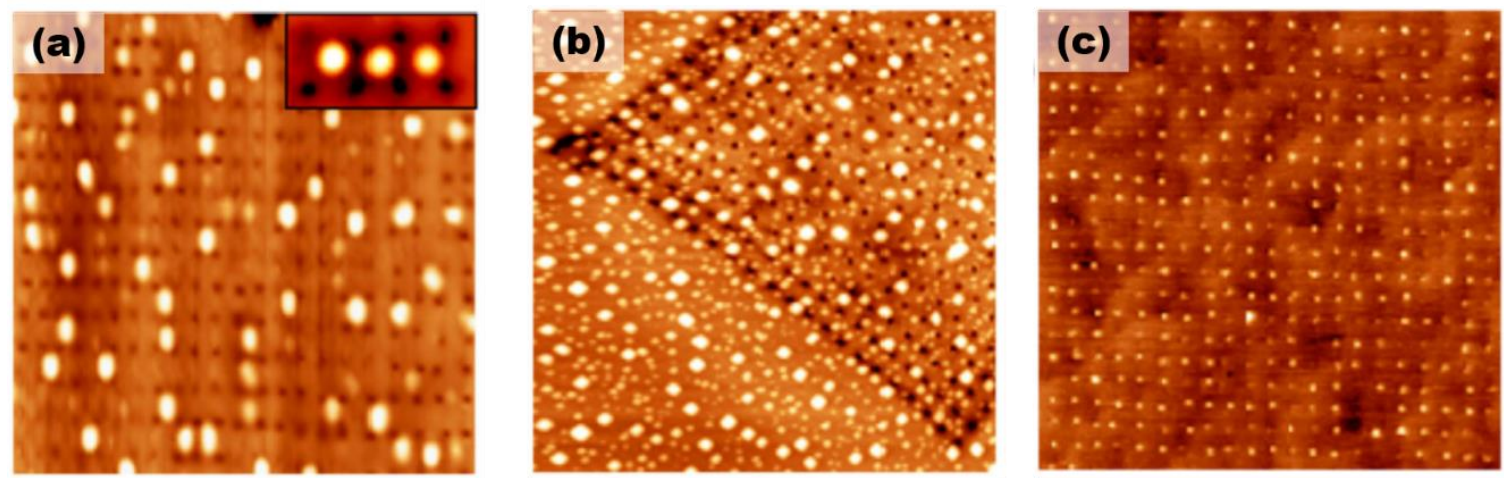

Figure 4-11: $2.5 \times 2.5 \mu \mathrm{m}^{2}$ AFM image showing the results for $1.4 \mathrm{~nm}$ of Ge deposited on patterned $\mathrm{Si}(001)$ substrate at different growth temperatures $\left(\mathrm{T}_{\text {growth }}\right)$. (a) $\mathrm{T}_{\text {growth }}=750{ }^{\circ} \mathrm{C}$ with pitch $=150 \mathrm{~nm}$ (inset shows the higher magnification image of 3 QDs localized on crown region), (b) $\mathrm{T}_{\text {growth }}=750{ }^{\circ} \mathrm{C}$ with pitch $=350 \mathrm{~nm}$, and (c) $\mathrm{T}_{\text {growth }}=550{ }^{\circ} \mathrm{C}$ with pitch $=150 \mathrm{~nm}$ showing QDs nucleating only in the pits. ${ }^{101}$

(001) terraces but becomes a continuously height modulated, quasi-sinusoidal profile.

Theoretical studies suggest that interactions between the substrate pattern wavelength and the intrinsic roughening wavelength becomes important for such profiles during the early stages of QD self-assembly process. Hu et al. ${ }^{119}$, using a relatively simple 2D theoretical model for a sawtooth-patterned substrate, showed that strain relaxation directs island nucleation to the trough (pit-bottom) but in the presence of large surface energy anisotropy, nucleation at both the trough and the crest are equally favorable. In contrast, the MonteCarlo simulation approach of Pascale et al. ${ }^{101}$ predicts the existence of an equilibrium configuration with islands nucleating in terraces in between the pits. Yang, et al., ${ }^{112}$ calculated the local chemical potential on patterned surface (ridges) and outlined that a local minimum exists at the crown region (concave) accounting to strain relaxation. To address the role of the WL, Vastola et al. ${ }^{113}$, from their theoretical model indicated that WL is highly relaxed near the pit edge (depending upon the pit inclination angle), which could 
result in extended residence time of diffusing atoms at the edge, therefore, increasing the probability of island nucleation at the edge instead of inside the pit.
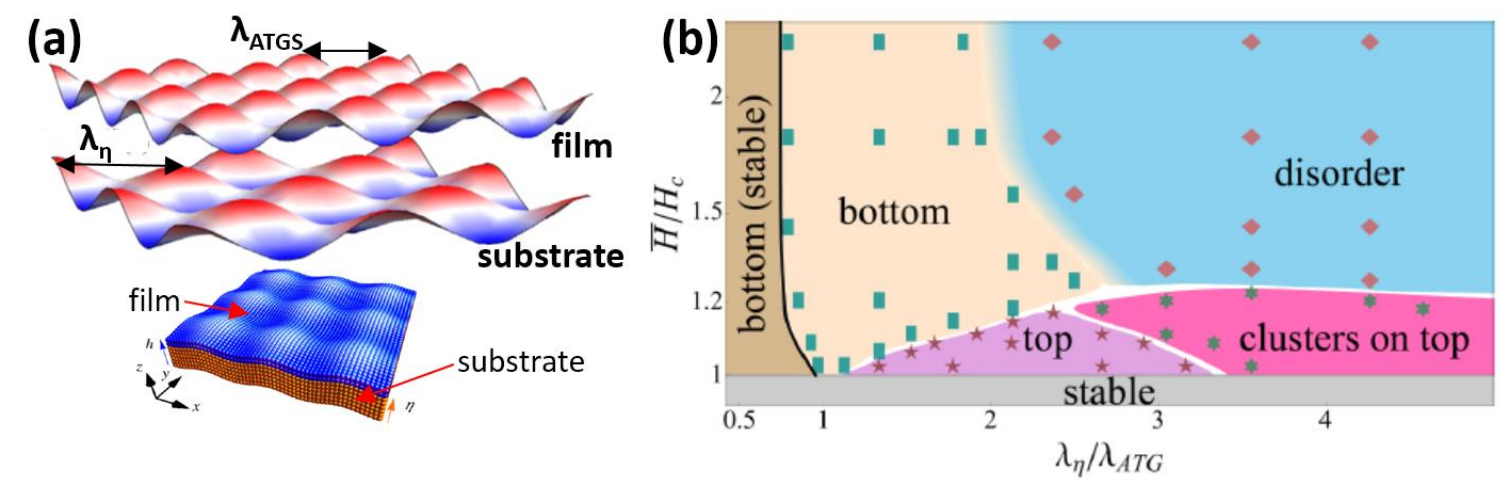

Figure 4-12: (a) $\lambda_{\eta}$ is the artificial wavelength imposed by pattern and $\lambda_{\text {ATGS }}$ is the intrinsic wavelength. (b) Phase space for growth location of Ge QDs on a quasi-sinusoidal pattern, $\mathrm{H}$ is the film thickness and $\mathrm{H}_{\mathrm{c}}$ is the wetting layer thickness. ${ }^{39,120}$

A recent continuum analysis by Aqua and $\mathrm{Xu}^{39,120}$ for strain-induced roughening, occurring specifically on a sinusoidal patterned surface, predicts a phase space for the preferred locations of QDs, see Figure 4-12. In the phase space plot, the abscissa is the ratio of the template wavelength to the ATGS wavelength, while the ordinate is the ratio of the film thickness to the critical wetting layer thickness (generally 3-4ML for pure Ge on $\mathrm{Si}(001)^{29}$ ). The model incorporates surface diffusion, anisotropic surface energy, surface chemical potential variations due to local elastic fields that vary along with local morphology, and the effects of the wetting layer. The model predicts a small region ("top") in their phase space where the localization of QDs occur at the crowns, provided that the film thickness is slightly larger than the critical wetting layer thickness and the substrate pattern wavelength is 1.2-3.3 times that of the instability wavelength. For large thickness, nucleation in the valleys ("bottom") is predicted. For template wavelengths less than $\lambda_{\mathrm{ATGS}}$ 
Ge is predicted to simply fill the valleys like water filling a cup ("bottom stable"), without higher angle surface facets.

Basically, the Aqua model predicts the island nucleation position based on the interaction between intrinsic lengthscales vs artificially imposed lengthscales (imposed by pattern) and surprisingly few experimental studies have directly addressed the effect of interaction between the pattern wavelength and $\lambda_{\text {ATGS. }}$ Here, in this dissertation work, we will analyze the results of our systematic experimental study of island positioning as a function of $\lambda_{\mathrm{ATGS}}$ on a modified surface with artificially imposed wavelength $\left(\lambda_{\eta}\right)$ of the pattern to validate the Aqua model. 


\section{Chapter 5: Directed self-assembly of $\mathrm{Si}_{1-\mathrm{x}} \mathrm{Ge}_{\mathrm{x}}$ QDs: 2D template}

\section{development}

As discussed in previous chapter, there are various lithographic techniques to pattern the growth substrate in order to direct the self-assembly process of QDs. In this Chapter, we discuss FIB- and EBL-based patterning approaches for creating the 2D pit-patterned templates. EBL was ultimately found to produce excellent and highly controllable patterns with good crystalline quality after the Si buffer growth.

\subsection{Preliminary work on FIB based patterning approach}

To generate templates for patterning the QDs, we first investigated the use of a Ga+ FIBto develop patterns on Si (001) substrates. The FIB was an FEI Helios 650 located at the NIST NanoFab. The workflow developed for FIB patterning was: (i) Use the FIB to write a pattern of defect sites by bombarding with a focused beam of $30 \mathrm{KV} \mathrm{Ga}^{+}$ions on $\mathrm{Si}(001)$,

(ii) Use wet chemical etching to clean and selectively etch the defect sites creating nanopitpatterns and (iii) deposit Ge and Si on the patterned substrate in MBE. This technique was developed in prior work in our group under separate funding, and the results initially seemed encouraging. ${ }^{121}$ However, results obtained at the end of that project led us to question whether directed self-assembly of Ge QDs was not actually driven by the imposed morphological pattern. Transmission electron microscopy results suggested that in at least some instances, residual crystal damage at the FIB sites, coupled with the highly constrained thermal budget during MBE cleaning and Ge growth (in order to preserve the fine-scale pattern), may have been the primary bias dictating Ge diffusion and QD site selection. ${ }^{121}$ Although QD patterning did occur, the incorporated damage made it difficult 
to subsequently cap the QDs and propagate into the $3^{\text {rd }}$ dimension so as to form the quantum dot mesocrystal (QDMC). As a result, in the initial experimentation for this project, we reexamined the process flow, particularly to examine whether the use of higher process temperatures would eliminate defectivity while retaining a surface morphological pattern.
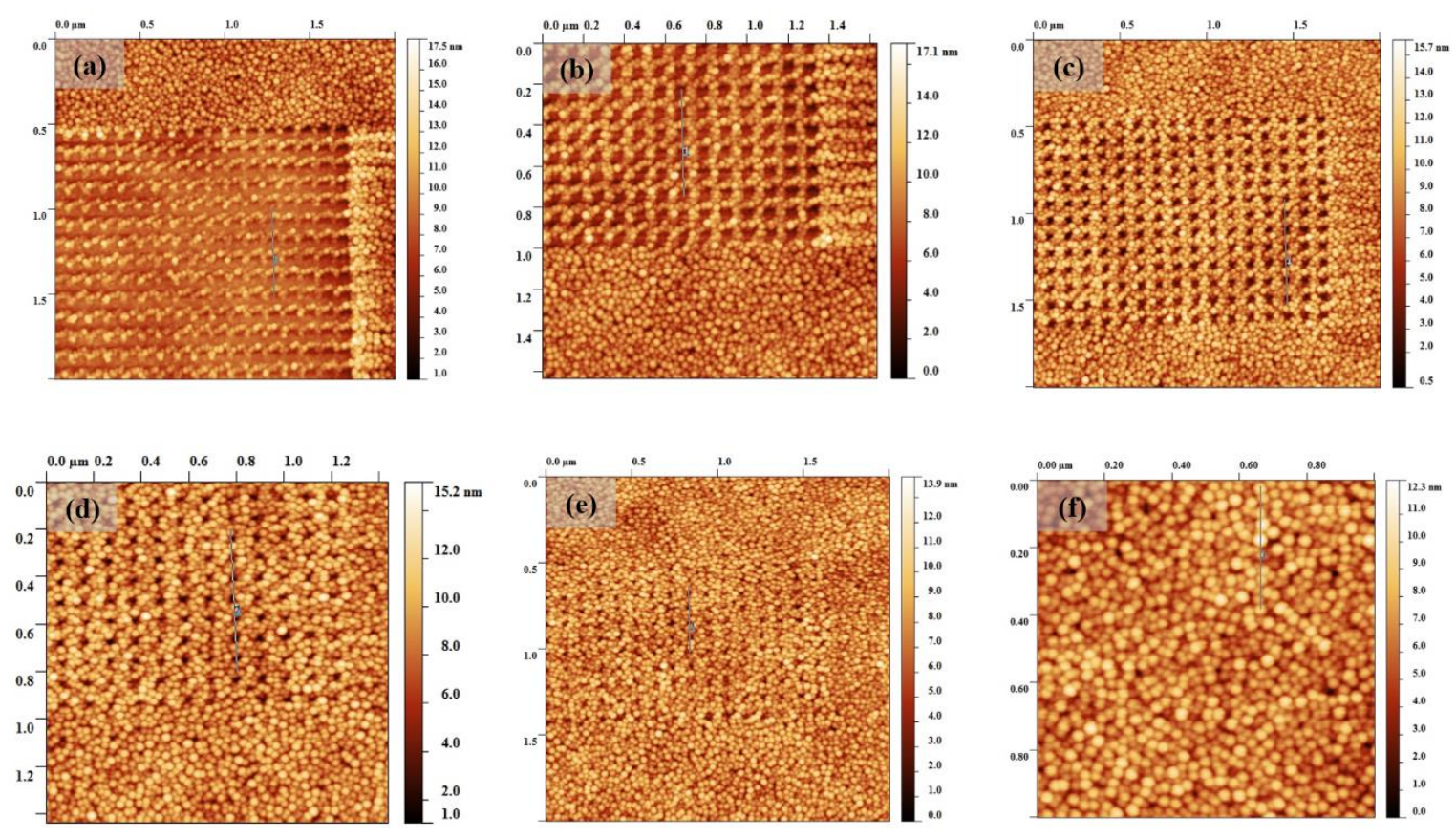

Figure 5-1: Topographic AFM images of 30kV energy Ga+ ion patterns for various ion dose with $8 \mathrm{ML}$ Ge (no Si buffer). (a) $20 \mathrm{~K}$ ions/site with $100 \mathrm{~nm}$ pitch, (b) $10 \mathrm{~K}$ ions/site with $100 \mathrm{~nm}$ pitch, (c) $5 \mathrm{~K}$ ions/site with $100 \mathrm{~nm}$ pitch, (d) $3 \mathrm{~K}$ ions/site with $100 \mathrm{~nm}$ pitch, (e) $2 \mathrm{~K}$ ions/site with $100 \mathrm{~nm}$ pitch, (f) off-pattern region

Using FIB, we patterned $\mathrm{Si}(001)$ substrates with a range of $\mathrm{Ga}^{+}$ion doses and energies with $50 \mathrm{~nm}$ and $100 \mathrm{~nm}$ pitch (pattern periodicity). Initially, the patterned substrates underwent standard chemical etch/clean with dip in buffered HF as final step to allow low temperature processing in MBE. Preliminary data suggested that for low temperature (less than $550^{\circ} \mathrm{C}$ ) cleaning process, routinely obtaining a clean surface was very challenging. Figure 5-1 shows on-pattern AFM scans from patterns processed at low temperature, 
created with $30 \mathrm{KV} \mathrm{Ga}^{+}$ions. Even though the patterns were well preserved during low temperature process cycle the results for $8 \mathrm{ML}$ Ge growth $\left(450^{\circ} \mathrm{C}\right)$ on the pattern suggested that good epitaxial growth was very challenging. Therefore, we started exploring regular high temperature $\left(\sim 800^{\circ} \mathrm{C}\right)$ process to clean the patterned wafer prior to the deposition of Ge.
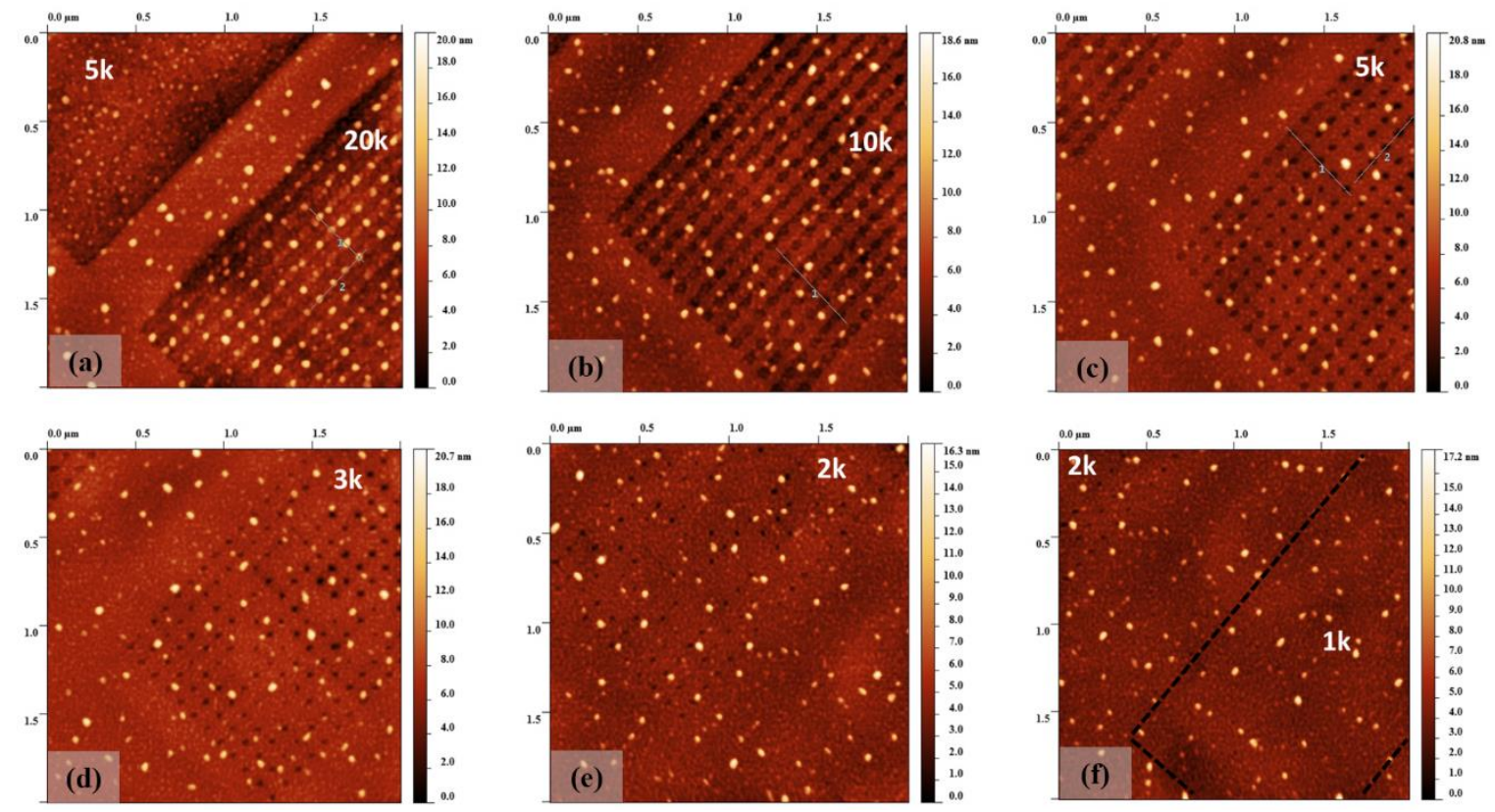

Figure 5-2: Topographic AFM images of 30kV energy Ga+ ion patterns for various ion dose. (a) $5 \mathrm{~K}$ ions/site with $50 \mathrm{~nm}$ pitch and $20 \mathrm{~K}$ ions/site with $100 \mathrm{~nm}$ pitch, (b) $10 \mathrm{~K}$ ions/site with $100 \mathrm{~nm}$ pitch, (c) $5 \mathrm{~K}$ ions/site with $100 \mathrm{~nm}$ pitch, (d) $3 \mathrm{~K}$ ions/site with $100 \mathrm{~nm}$ pitch, (e) $2 \mathrm{~K}$ ions/site with $100 \mathrm{~nm}$ pitch, (f) $1 \mathrm{~K}$ ions/site with $100 \mathrm{~nm}$ pitch

During high-temperature cleaning prior to insertion into the MBE, the passive $\mathrm{SiO}_{\mathrm{x}}$ layer created by ex situ chemical cleaning process was desorbed in situ at $800^{\circ} \mathrm{C}$ after an overnight temperature ramp, plus $5 \mathrm{hr}$. prebake at $600{ }^{\circ} \mathrm{C}$. The substrate was then cooled to desired temperature for Si buffer layer growth followed by Ge deposition. Figure 5-2 shows on-pattern AFM scans from patterns created with $30 \mathrm{kV} \mathrm{Ga}^{+}$ions. The patterned substrate underwent an oxide desorption at $800{ }^{\circ} \mathrm{C}$ followed by growth of $8 \mathrm{ML}$ of $\mathrm{Ge}$ at 
$450{ }^{\circ} \mathrm{C}$. Ion doses were varied in each pattern site as shown. The pattern with $50 \mathrm{~nm}$ pitch was macroscopically etched during the wet-chemical treatment, see Figure 5-2(a). The macroscopic etching, wherein the entire FIB pattern is etched away by the cleaning process, leaving a broad valley, is the result of the overlapping of defect cascade regions during the FIB process. Reducing the ion energy from $30 \mathrm{kV}$ to $8 \mathrm{kV}$ could help reduce this effect, however, our preliminary data shows little or no pattern preservation for $8 \mathrm{kV}$ patterns, see Figure 5-3. The best pit-pattern observed was formed at $30 \mathrm{kV}$ with 5000 ions/site dose and $100 \mathrm{~nm}$ pitch, see Figure 5-2(c). The overall QD pattering was quite poor with dots mostly observed to nucleate randomly with no preference in site-selection especially for the pit-bottom as reported in literature. One possible reason for Ge to avoid the pits could be due to the defected sites at the bottom of pits from FIB process.
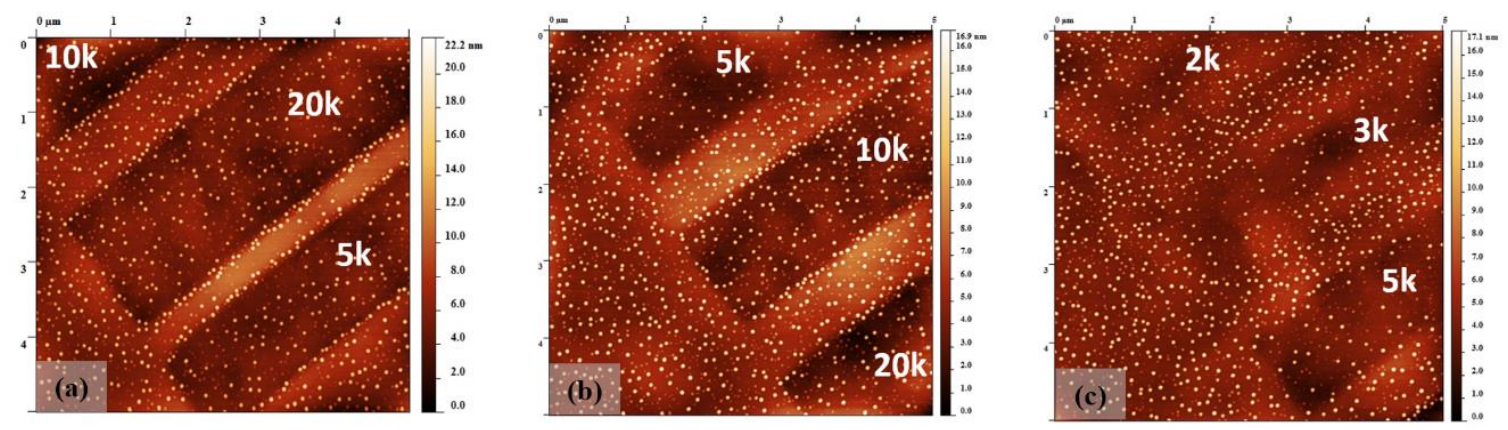

Figure 5-3: Topographic AFM images of $8 \mathrm{kV}$ energy Ga+ ion patterns with (a) $5 \mathrm{~K}$ ions/site with $50 \mathrm{~nm}$ pitch and $20 \mathrm{~K}$ ions/site with $100 \mathrm{~nm}$ pitch; (b) $10 \mathrm{~K}$ ions/site with $100 \mathrm{~nm}$ pitch and $5 \mathrm{~K}$ ions/site with $100 \mathrm{~nm}$ pitch; (c) $5 \mathrm{~K}$ ions/site with $100 \mathrm{~nm}$ pitch, $3 \mathrm{~K}$ ions/site with $100 \mathrm{~nm}$ pitch and $3 \mathrm{~K}$ ions/site with $100 \mathrm{~nm}$ pitch

From the standpoint of both epitaxial growth and electronic properties, it is desirable to grow a Si buffer on the patterned substrate surface. This must retain the underlying morphology while presenting a growth surface with excellent crystalline quality for QD self-assembly. We investigated the effect of $\mathrm{Si}$ buffer layer growth at different 
temperatures. A $15 \mathrm{~nm}$ thick Si buffer layer deposited at $700{ }^{\circ} \mathrm{C}$ almost entirely washed out the patterned region with only macroscopic etch marks observed for higher doses, see Figure 5-4(a). Reducing the Si buffer layer thickness to $10 \mathrm{~nm}$ and further reducing the growth temperature to $600{ }^{\circ} \mathrm{C}$ was also not able to preserve the FIB patterns, see Figure 54(b). One aspect of these results is particularly confusing - if residual defectivity exists at the pit sites as indicated by the inability of Ge to nucleate in the pits, we would expect, based on prior research, that the Si would also avoid the pits. However, the results of Figure 5-4 suggest preferred sticking of Si in the pits, resulting in planarization.
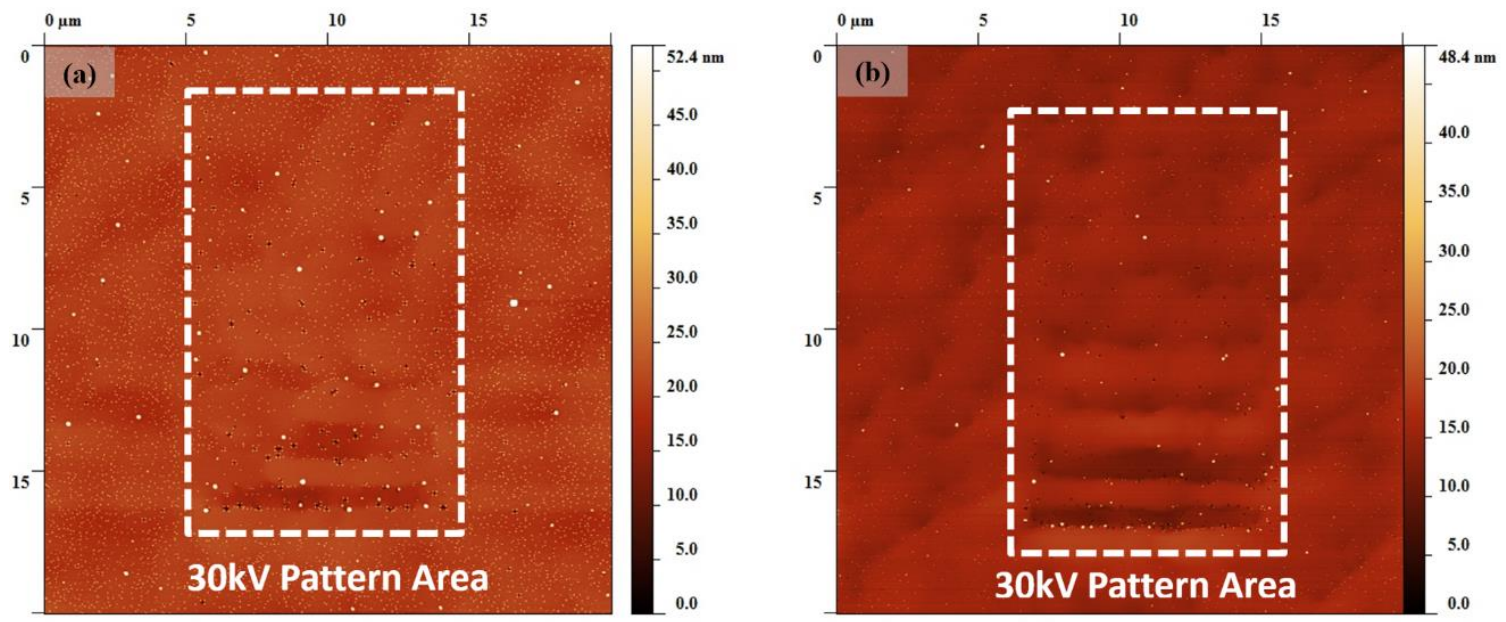

Figure 5-4: Topographic AFM images of $30 \mathrm{kV}$ energy Ga+ ion patterns with (a) $15 \mathrm{~nm} \mathrm{Si}$ buffer at $700{ }^{\circ} \mathrm{C}$ with $6 \mathrm{ML} \mathrm{Ge}$ at $600{ }^{\circ} \mathrm{C}$; (b) $10 \mathrm{~nm}$ Si buffer at $600{ }^{\circ} \mathrm{C}$ with $6 \mathrm{ML}$ Ge at $600{ }^{\circ} \mathrm{C}$

Given these issues, and our rising concern about the repeatability of the FIB-based process, we opted to instead develop an EBL-based patterning to initiate and direct the QDs selfassembly process. 


\subsection{D patterning with EBL}

Fabrication of 2D templates underlies the entire QD patterning process. Therefore, it is very important to understand the process and science of the patterning technique being used. In this work, we have used EBL to pattern 2D square lattice templates on a $\mathrm{Si}(001)$ substrate, but more complicated 2D templates can also be created from similar processing. EBL is a fundamental nanofabrication technique, enabling patterning technologies to create masks and templates. Some aspects of EBL processing were already discussed in Chapter 2. In short, the workflow used for the EBL based patterning approach here is: (i) Coat a masking resist layer on $\mathrm{Si}$ (001), (ii) use EBL to expose resist/mask layer on specified regions of the substrate, (iii) develop the substrate to create pattern on the resist mask, and (iv) perform reactive ion etching (RIE) to transfer the patterns on to the substrate

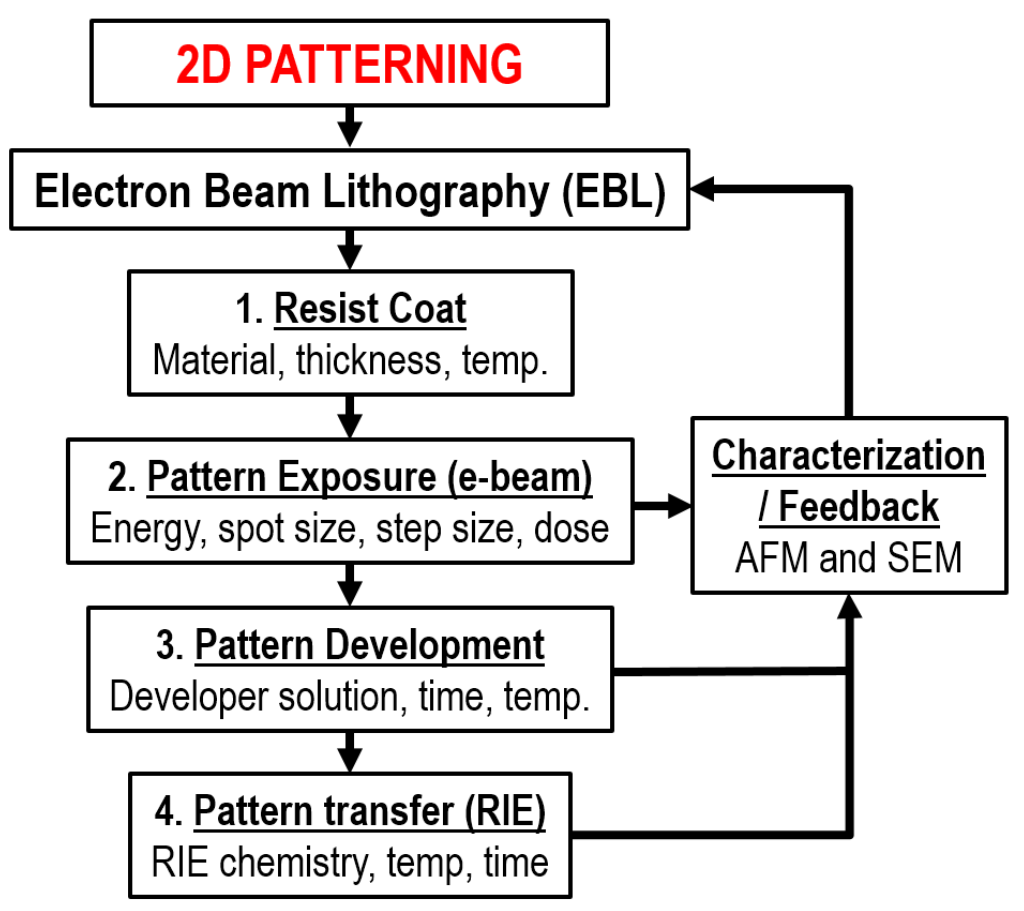

Figure 5-5: Schematic for the workflow used for EBL based patterning approach optimization. 
and then clean mask layer leaving just a patterned substrate, see Figure 5-5 and Chapter 2 for more details. As outlined in the Figure 5-5, there are numerous variables within the EBL patterning process that need to be optimized for successful fabrication of $2 \mathrm{D}$ templates. In this section, we discuss in detail the entire EBL patterning process, recipe and optimization work for successfully creating $2 \mathrm{D}$ pit-pattern templates on a $\mathrm{Si}(001)$ substrate. Table 5-1 lists some of the important parameters that require optimization to achieve a high resolution, high quality pattern with large process windows to maximize yield and reproducibility.

Table 5-1: Parameters affecting the EBL process ${ }^{122}$

\begin{tabular}{|c|l|c|}
\hline S/N & \multicolumn{1}{|c|}{ Parameter } & Process impact \\
\hline 1 & Resist material & Resolution \\
\hline 2 & Resist thickness & Resolution, pattern quality \\
\hline 3 & Beam/ Exposure energy & Resolution, proximity effect \\
\hline 4 & Exposure (dose) & Pattern quality \\
\hline 5 & Developer solution & Sensitivity, resolution \\
\hline 6 & Development time and temperature & Resolution \\
\hline
\end{tabular}




\subsubsection{Resist}

The e-beam exposure strongly depends on type of resist and substrate being used. There are basically two types of resist - positive-tone and negative-tone. The solubility is increased upon exposure to electrons in a positive-tone resist, whereas the solubility decreases upon exposure in negative-tone resist. For our experiments, we used a positivetone resist - PMMA (poly-methyl methacrylate) which is a long chain polymer. PMMA is by far the most popular e-beam resist, offering extremely high resolution $\left(\sim 10 \mathrm{~nm}^{67}\right)$ and ease of handling which is well suited for our proposed work. Further, there are various types of PMMA resist based on the mean molecular weight. The most commonly used are have masses of 495,000 and 950,000 molecular weight resins in either chlorobenzene anisole, which is a safer solvent.

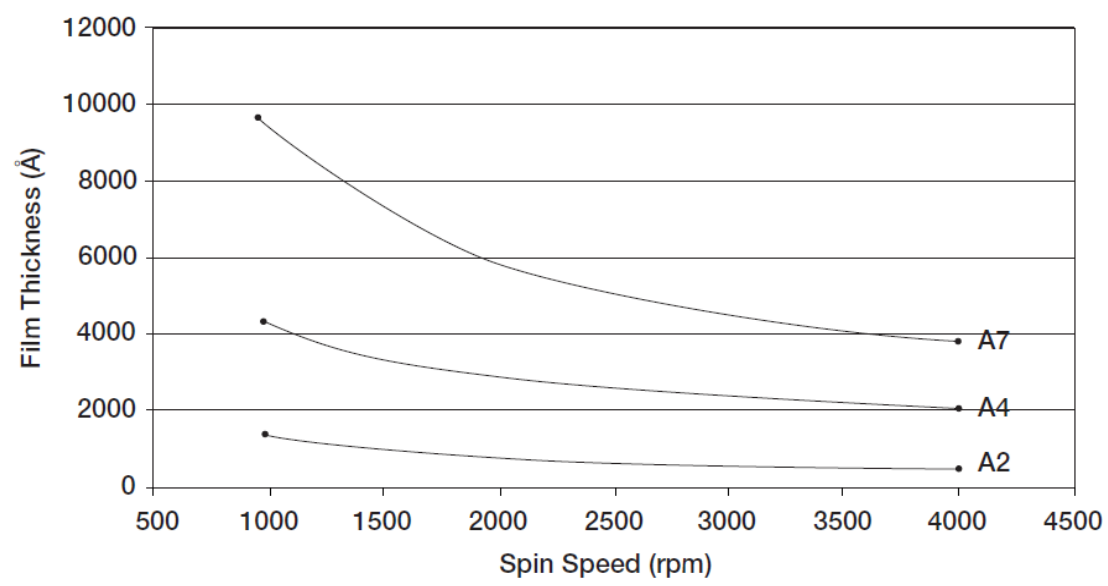

Figure 5-6: 950K PMMA-A resists spin curve, solids: $2 \%$ - 7\% in Anisole. ${ }^{145}$

For our purpose, a 2 in. diameter, $250 \mu \mathrm{m}$ thick, undoped $\mathrm{Si}(001)$ substrate is cleaned in successive rinses of acetone and methanol, prior to spin coating the resist. A $50-70 \mathrm{~nm}$ thick, 950K PMMA-A2 film is then spin-coated (6000 rpm for 30sec) followed by baking at $180^{\circ} \mathrm{C}$ for $90 \mathrm{sec}$ on a hot plate, see Figure 5-6 showing the spin curve for 950K-PMMA- 
A2 resist. The bake temperature of resist is critical as higher temperature results in cracking and degradation of resist quality.

The thickness of the resist in combination with the energy of the incident beam is very important for the success of entire process. For instance, at lower energy the forward scattering of incident electrons can spread and degrade the pattern quality. Increasing the energy of the incident beam can reduce the broadening effect but at very high energies, the backscattering of electrons from the resist-substrate interface may cause exposure far from the intended region, see Figure 5-7. ${ }^{123,124}$ The pattern density also plays an important role in fine-tuning the balance between the forward and backscattering of the incident electrons. This is also termed as proximity effect ${ }^{125}$ where the undesired scattering events of incident electrons causes pattern distortion and overexposure.
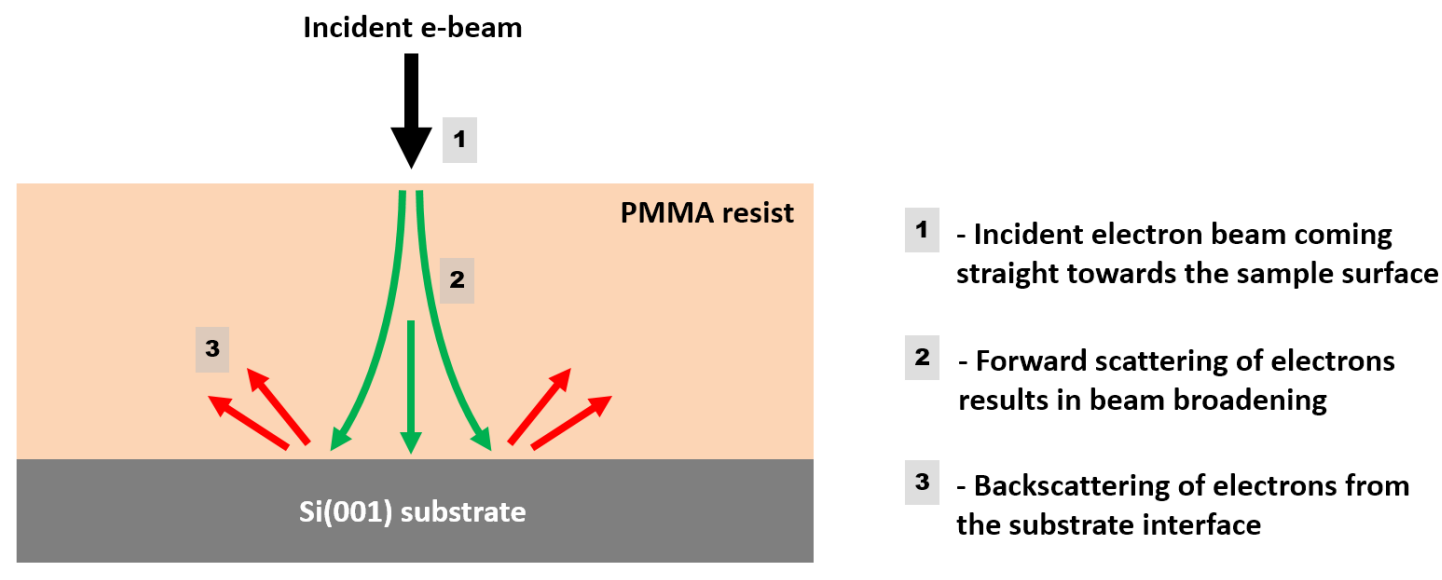

Figure 5-7: Forward and backscattering of incident electrons during resist exposure.

The doping level of the substrate can also effect the pattern quality as highly resistive substrate can build an undesired charge concentration leading to the proximity effect. However, we did not observe any effect of using doped vs undoped $\mathrm{Si}(001)$ substrate during our patterning work. But it should be noted that at lower pattern pitch (close to the 
resolution limit of PMMA) the substrate doping level will play a critical role in deciding the pattern quality. Figure 5-8 shows the proximity effect when patterning a $500 \mathrm{~nm}$ pitch pattern at different e-beam energy.
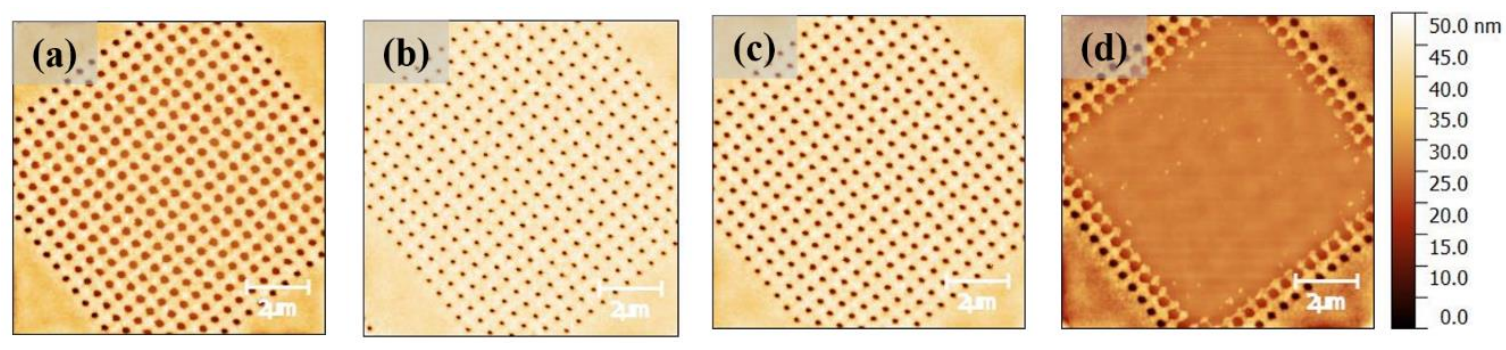

Figure 5-8: $500 \mathrm{~nm}$ pitch pattern exposed using Raith-50 at a constant dose with spot size 2 and beam energy of (a) $10 \mathrm{kV}$, (b) $15 \mathrm{kV}$, (c) $20 \mathrm{kV}$ and (d) $25 \mathrm{kV}$. At lower and higher beam energy, the beam broadening effect is prominent.

Figure 5-8 shows the effect of beam energy variation but similar proximity effect is evident during PMMA thickness optimization as well. As described in Chapter 2, all of our higher resolution patterns with pitch 75-200 $\mathrm{nm}$ were performed in a Raith e-line instrument at the Univeristy of Maryland, and further optimization work for patterning was performed with 50-60 nm thick 950K PMMA-A2 and $10 \mathrm{kV}$ (30 $\mu \mathrm{m}$ aperture) of beam energy.

\subsubsection{Exposure and EBL dose optimization}

Patterns were made in either a standard CAD software or the Raith Elphy-Quantum software where dose and pitch are defined. The pitch of the pattern $\left(\lambda_{\mathrm{p}}\right)$ was varied from $50-300 \mathrm{~nm}$ and the electron dose was optimized independently for each pitch. A typical pattern is shown in Figure 5-9, consisting of constant dose test field and dose variation test field. In constant dose test field, multiple $5 \times 5 \mu \mathrm{m}^{2}$ pattern regions were exposed such that dose and pitch was constant within the $5 \times 5 \mu \mathrm{m}^{2}$ pattern region, see Figure 5-9(b). Whereas 
in dose variation test field, typically pattern regions were $5 \mathrm{x} \sim 10-25 \mu \mathrm{m}^{2}$ in size, dose was continuously varied from bottom to top with pitch remaining constant see Figure 5-9(b).

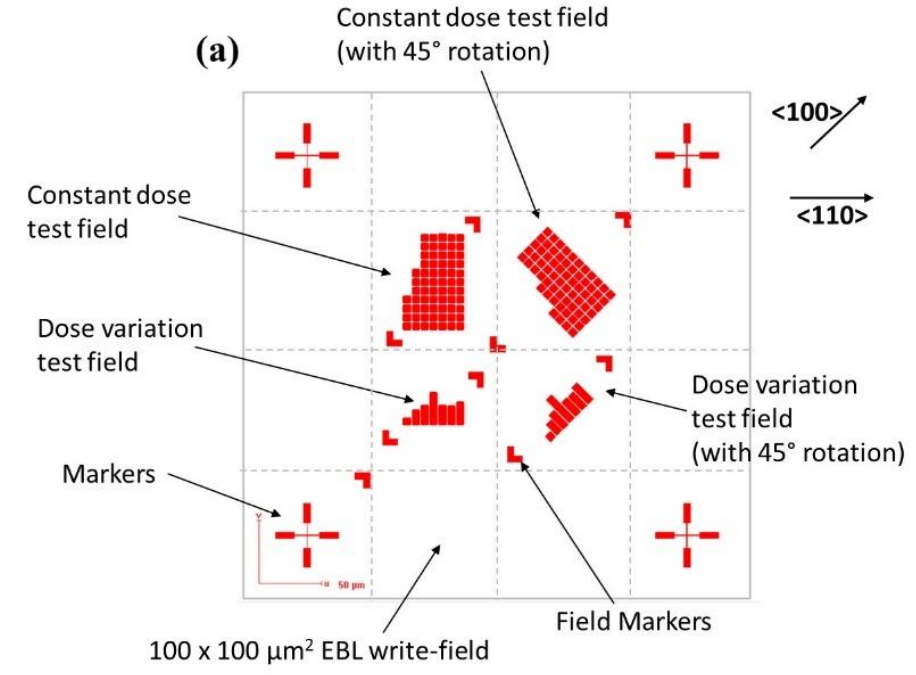

(c)

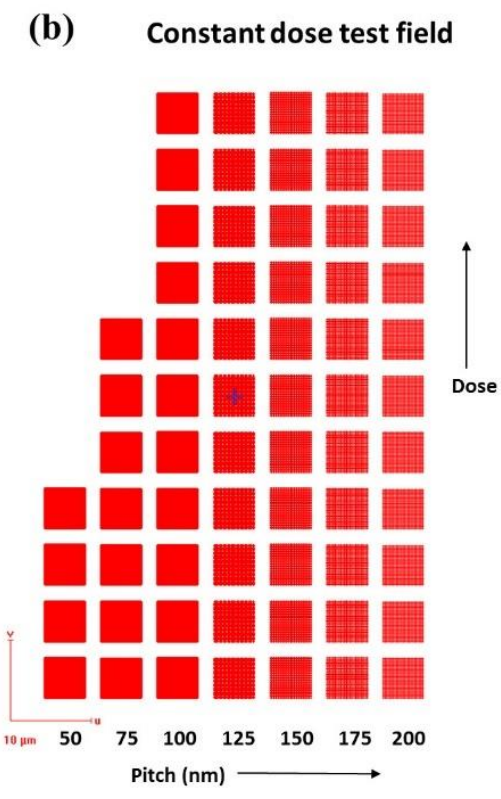

Dose variation test field

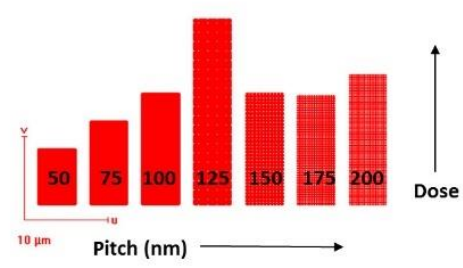

Figure 5-9: (a) Dot-pattern generated with Elphy Quantum Raith software for e-beam exposure showing (b) constant dose test field and dose variation test field along with (c) an optical image of the patterned region after development stage

The patterns used here are dot patterns, where only a "dot-pixel" is exposed to create a pit during etching. The pit opening width, W, scales with the electron dose at the dot-pixel; however, there is a critical dose beyond which adjacent pits overlap, degrading the pattern quality. As electron dose controls the pit opening, the optimum dose depends upon the 
pattern pitch as well, therefore careful optimization of dot dose is required to yield highly uniform and repeatable patterns.

Checking the dose variation test field post development is the quickest method of calibrating the dot dose. Figure 5-10 shows the $75 \mathrm{~nm}$ and $100 \mathrm{~nm}$ pitch pattern where dose was varied. The developed pattern clearly shows three distinct regions - (1) under-dose region, (2) ideal dose region and (3) over-dose region. Comparing the AFM micrograph with simulated dose patterns from Raith-Elphy Quantum software, we were able to calculate the idea dose range for each pattern as shown.

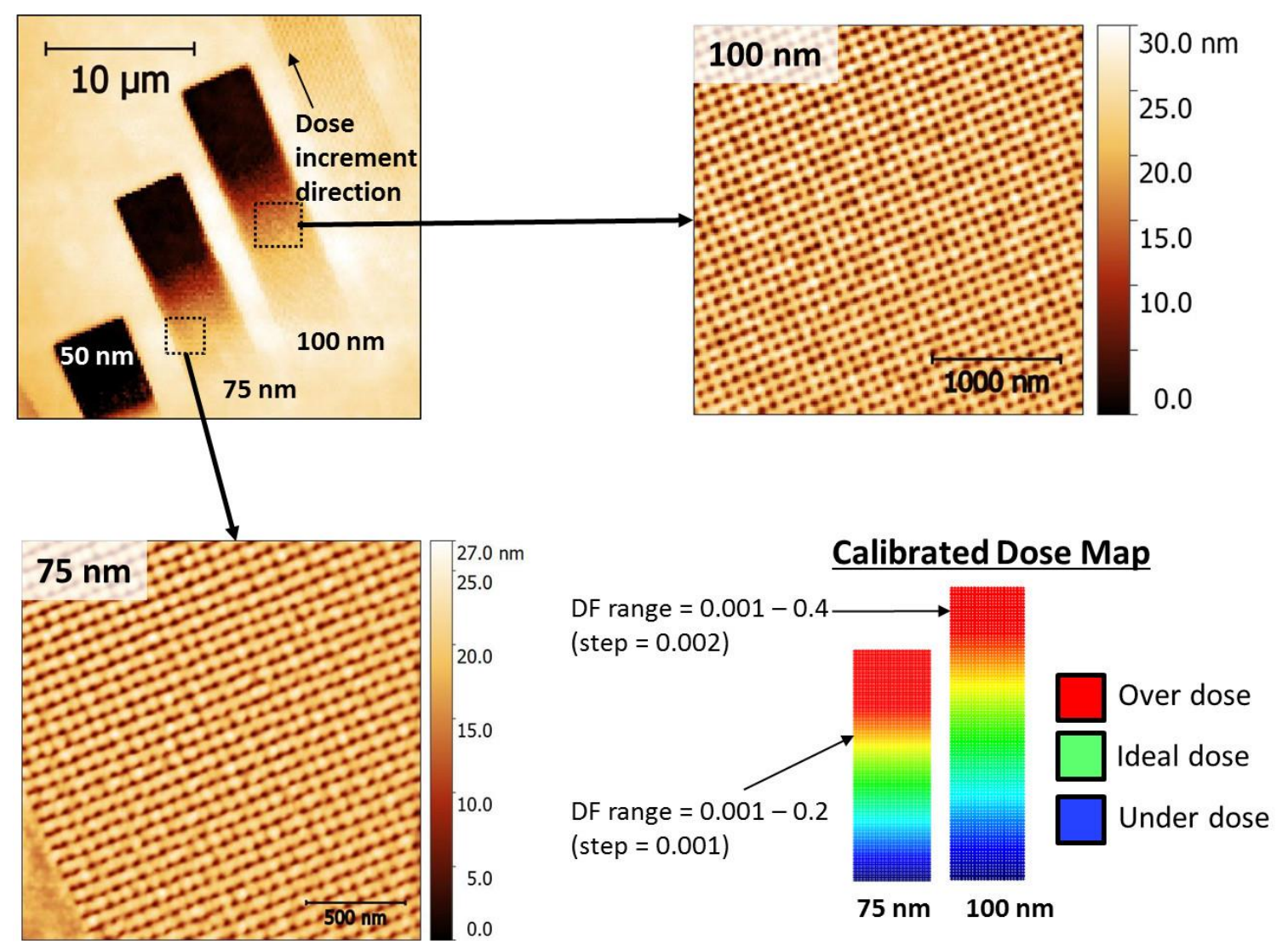

Figure 5-10: Dose variation test field showing AFM micrograph of 75 and $100 \mathrm{~nm}$ pattern along with calibrated color dose range map comparing three distinct regions. 
Patterns in the constant dose test field were also used to characterize the dose effect. As the dose was constant within each pattern we were able to correlate pit opening with the dose amount, see Figure 5-11. With the power curve fitting of the data, the relation obtained was

$$
(\text { Pit opening })=\mathrm{A} *(\text { Dot dose })^{0.37},
$$

where $\mathrm{A}$ is a fitting constant which was calculated to be 62.95 for our dose range. The plot clearly shows that beyond $10 \times 10^{-3} \mathrm{pC}$ of dose the relation is nominally linear. However, at lower dose the non-linearity is prominent which is basically a result of proximity effect.

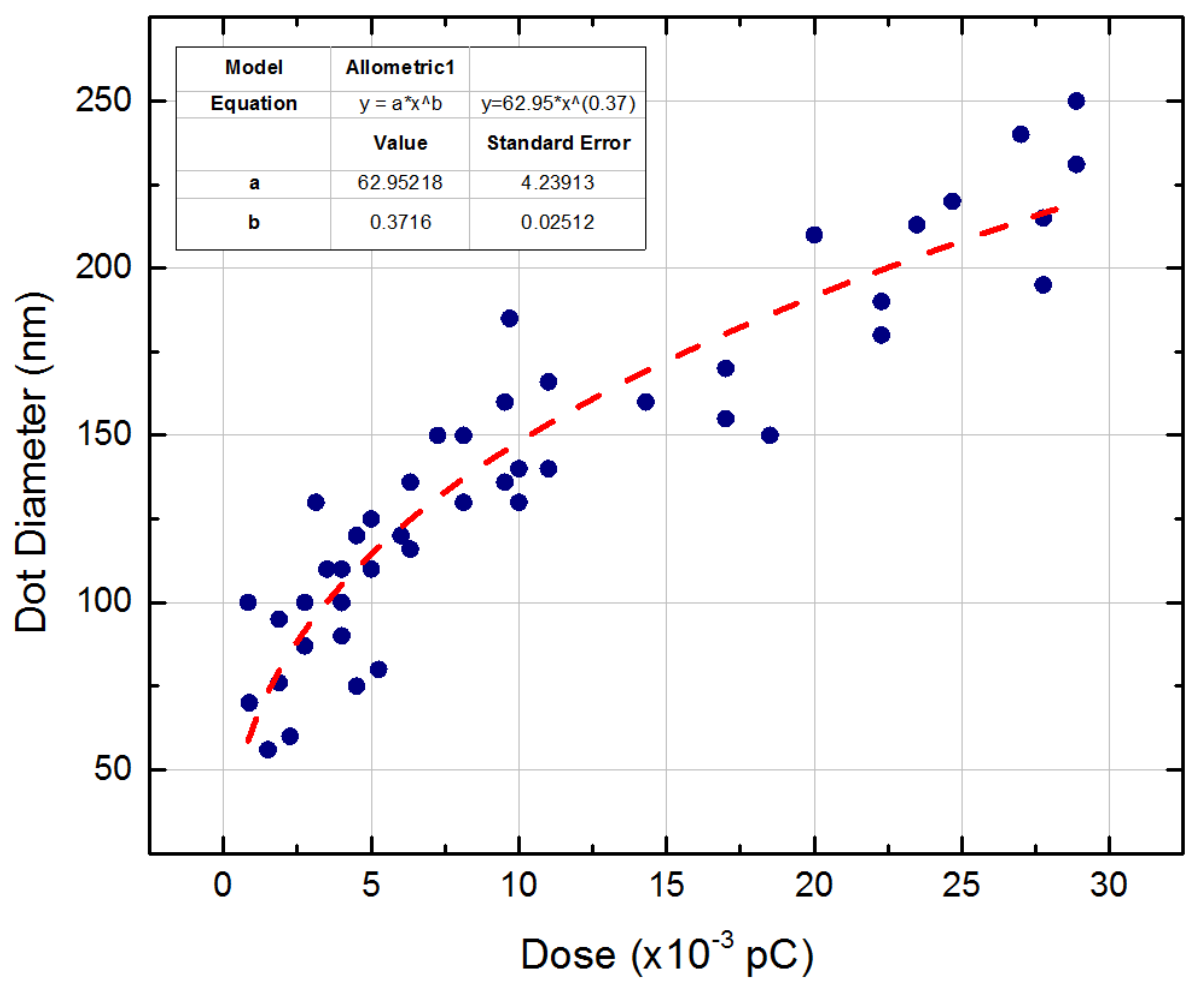

Figure 5-11: Plot of Dot diameter or pit opening vs. dot dose. Blue dots are the actual data and red dashed line is the fitted curve. Dot diameter is the pit opening width. 
(a) $\lambda=75 \mathrm{~nm}$
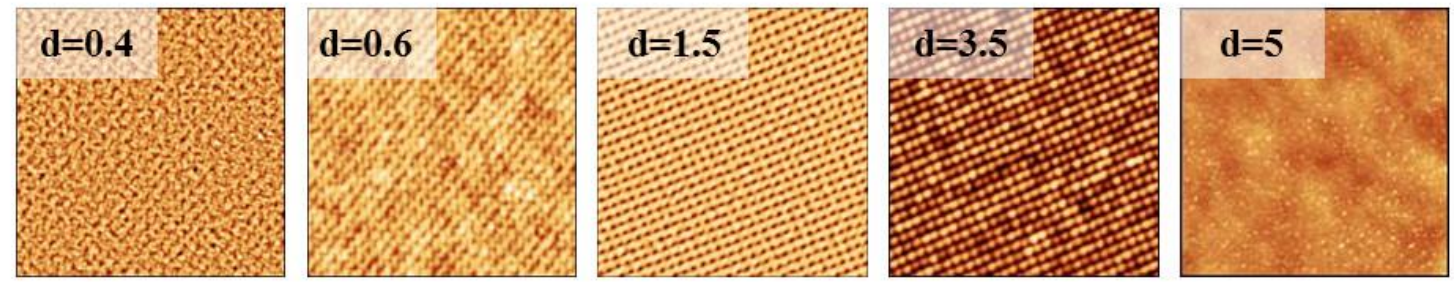

(b) $\lambda=100 \mathrm{~nm}$
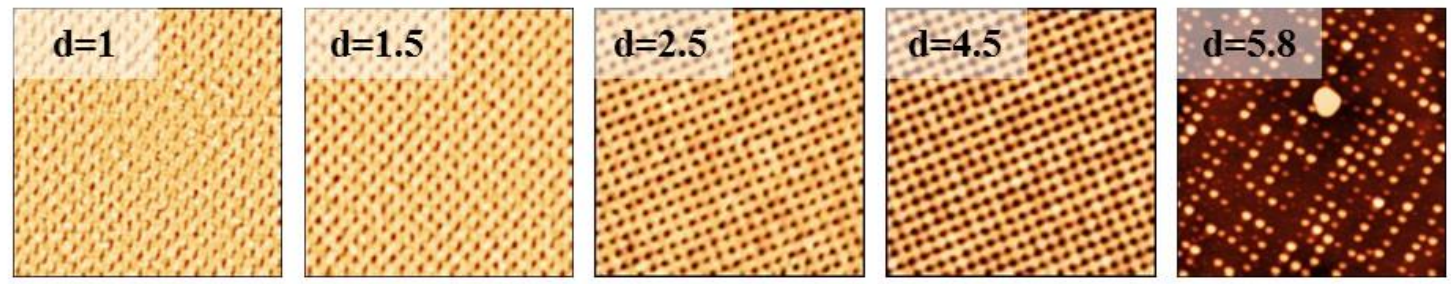

(c) $\lambda=150 \mathrm{~nm}$
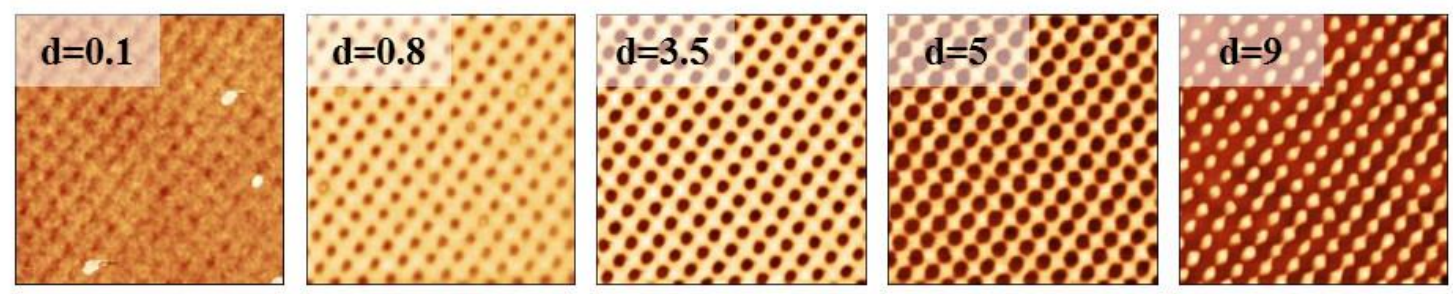

(d) $\lambda=200 \mathrm{~nm}$
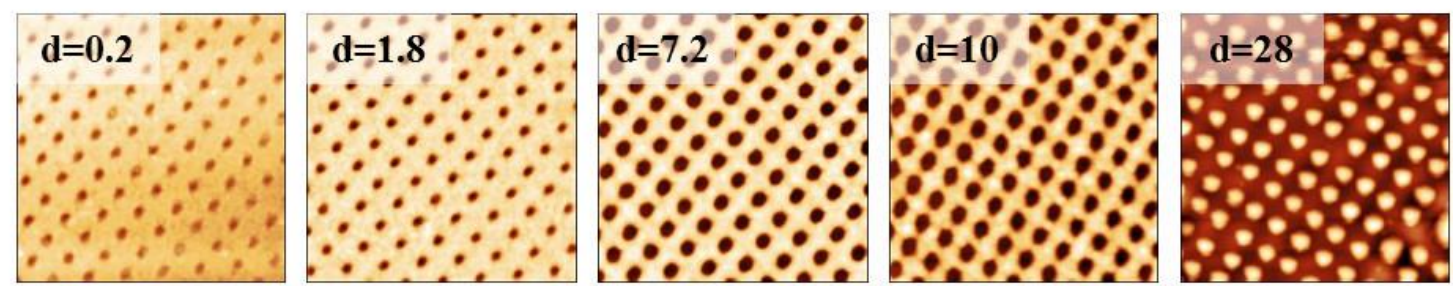

(e) $\lambda=300 \mathrm{~nm}$
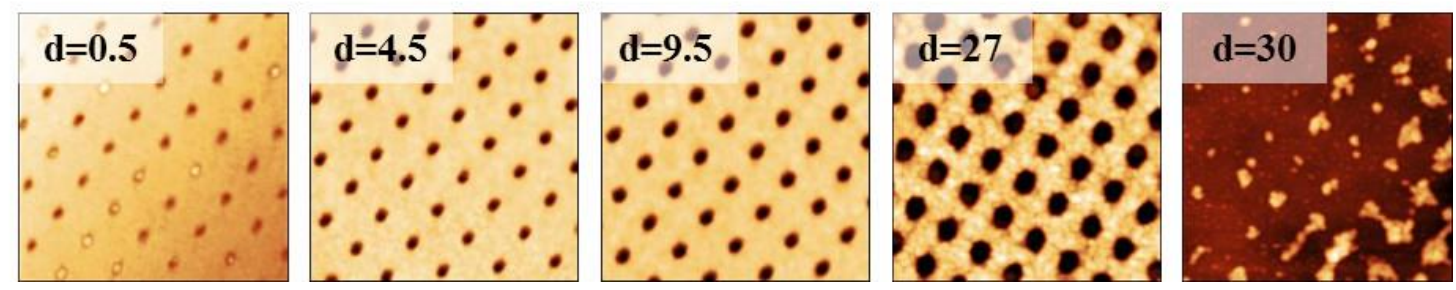

Figure 5-12: $2 \times 2 \mu \mathrm{m}^{2}$ AFM images showing the effect of dose change at various pitch. Dose (d) is increasing from left to right and is given in units of $10^{-3} \mathrm{pC}$. 
Figure 5-12 presents the AFM images taken from the constant dose test field showing the effect of varying dose. From the figure, it is clear that the quality of the pattern is strongly dependent on both the exposure dose and the pitch where the ideal dose varied along with the pitch value. For instance, $1.5 \times 10^{-3} \mathrm{pC}$ was observed to be the ideal dose at $75 \mathrm{~nm}$ pitch with a very narrow window of dose where the pattern would be of high quality. Whereas, for $300 \mathrm{~nm}$ pitch the dose window was wide enough where best patterns were observed between $4.5 \times 10^{-3}-27 \times 10^{-3} \mathrm{pC}$. For the lowest dose regions, the dose was insufficient in exposing all the PMMA through the resist depth. For the higher dose regions, the excessive exposure of the resist resulted in degradation of the patterns as shown in the figure at

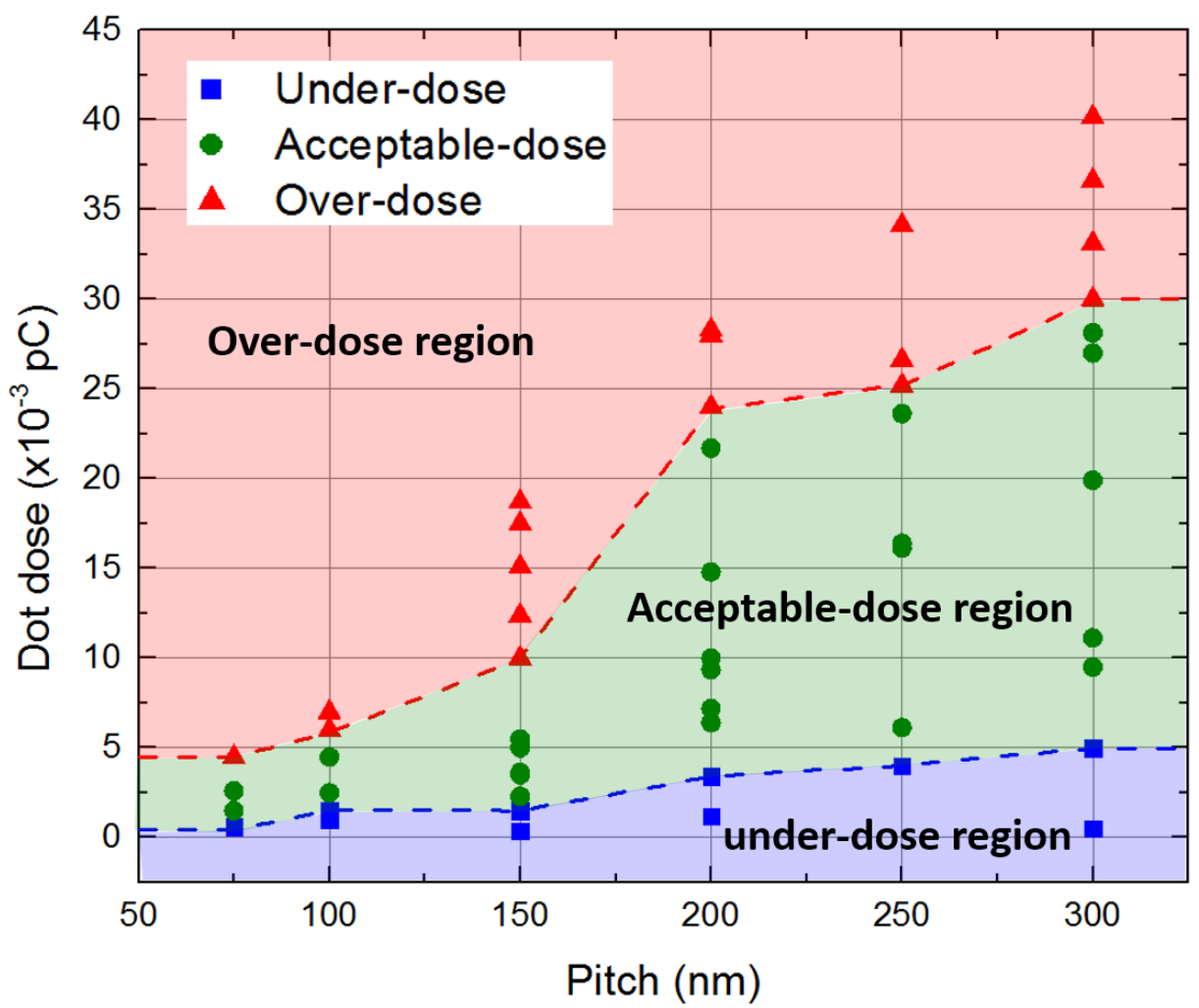

Figure 5-13: Plot of dot dose vs pattern pitch. The symbols represent the actual data with boundaries separating the three distinct regions observed experimentally during exposure - (1) under-dose region: result of insufficient clearance of the resist, (2) acceptable/idealdose region, and (3) over-dose region: excessive dose or exposure of pattern resulting in pattern degradation. 
extreme dose regions. Figure 5-13 summarizes the observed relation of dose and pitch outlining the process window. The acceptable dose window is wide at higher pitch and as we move to densest pattern with finer pitches the dose window narrows. Again, the narrowing of dose window is directly related with the proximity effect which is dominant at finer pitch patterns. Ideally, a large dose window is desired as fabrication process will then be less sensitive to process variables and has a better reproducibility.

\subsubsection{Pattern development}

During the exposure, the collision of electrons with the resist results in the breaking of long chain PMMA polymer into smaller fragments, see Figure 5-14. The average fragment size decreases with an increase in electron dose, resulting in higher solubility as smaller fragments more readily dissolves in developer solution. Due to scattering, dose varies spatially within the resist and so does the fragment size, see Figure 5-14(c). Therefore, the distribution of fragment size becomes important in understanding the development behavior.

(a)

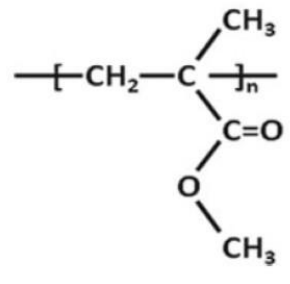

(b)

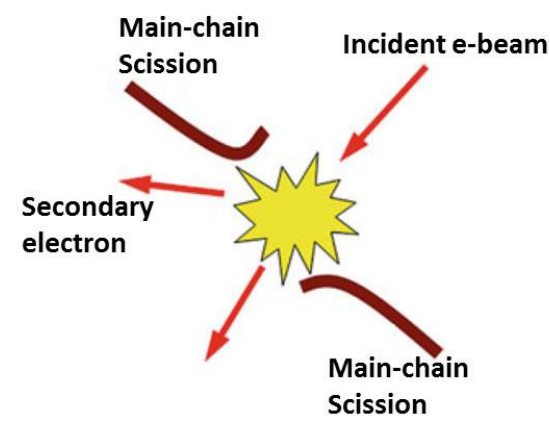

(c)

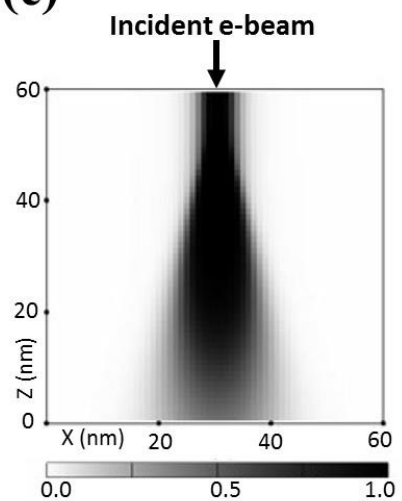

Figure 5-14: (a) Polymer sub-unit of PMMA, (b) fragmentation of polymer chain during EBL exposure, and (c) Spatial distribution of volume fraction of small fragments (less than 10 monomers) within the resist during single point exposure. ${ }^{122}$ 
After EBL exposure, the patterns are developed in a standard 1:3 MIBK:IPA (methyl isobutyl ketone: isopropyl alcohol) developer followed by DI rinse. The developer simply dissolves the smaller fragments of the resist (occurs in region of exposure for positive tone resist - PMMA), printing the desired pattern on the resist. The ratio of the developer solution can be fine-tuned to adjust the dissolving power, such that increasing the MIBK fraction will result in more powerful solvent which can remove comparatively longer polymer fragments. However, powerful solvents are not desirable to yield high resolution patterns as their polymer dissolution rate and selectivity is much harder to control.

The development time and temperature of the solution is important as leaving the sample too long or at higher process temperature can overdevelop the patterns degrading the quality. Developing time and temperature depends upon the type of resist, resist thickness, exposure dose and developing solution. Currently, for quality patterns, a 6-10 sec development time at room-temperature was found to be ideal in producing patterns with pitch $>75 \mathrm{~nm}$. For finer pitch $(\leq 75 \mathrm{~nm})$, a cold temperature $\left(<5^{\circ} \mathrm{C}\right)$ development of the exposed pattern is required. ${ }^{116,126}$ Figure $5-15$ shows a $50 \mathrm{~nm}$ pitch pattern where the pattern development was performed at room-temperature and at a developer temperature of -10 ${ }^{\circ} \mathrm{C}$, with process time of $10 \mathrm{sec}$. The lower temperature basically increases the dose window (experimentally it is found that lowering the development temperature to $-10{ }^{\circ} \mathrm{C}$ results in an increase of dose window by almost a factor of 5$)^{122}$ by controlling the diffusion kinetics of solvent molecular mobility, improving the selectivity and enabling the production of higher resolution patterns. Cold temperature of the developer solution can be attained either by using a chiller or by using a salt-ice-water bath. For the work performed here, we did 
not further pursue the low temperature development as the pitch of our test patterns were $>75 \mathrm{~nm}$ and RT development was good enough to yield uniform patterns.
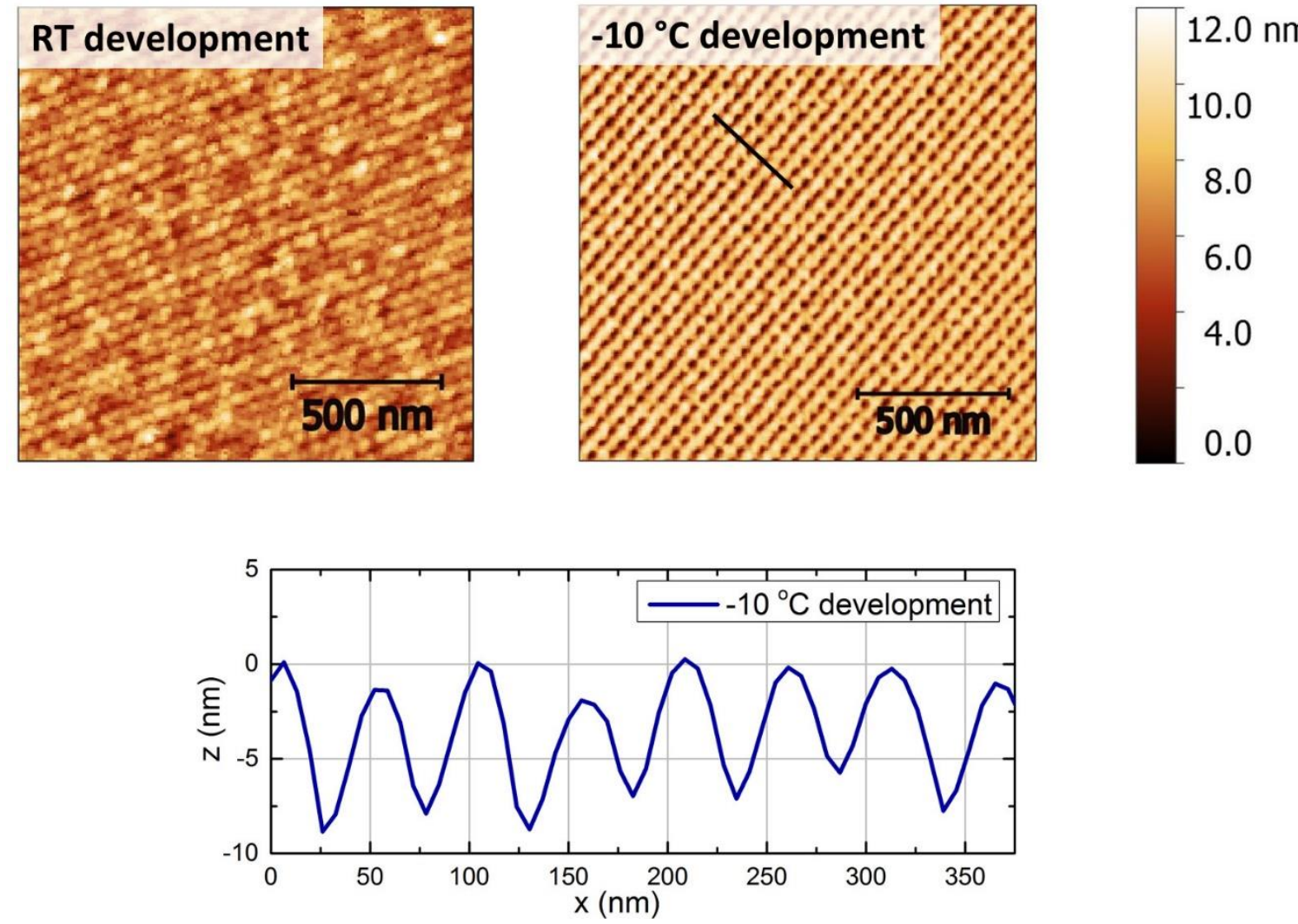

Figure 5-15: $1.5 \times 1.5 \mu \mathrm{m}^{2}$ AFM image with $50 \mathrm{~nm}$ pitch. The patterns were developed at room temperature (RT) and at $-10{ }^{\circ} \mathrm{C}$ with a development time of $\sim 10 \mathrm{sec}$. The pattern resolution and contrast is clearly enhanced by the low temperature development process. The line scan is taken from the $-10{ }^{\circ} \mathrm{C}$ development sample. 


\subsubsection{Pattern transfer and resist removal}

After development, the pattern is then transferred to the Si substrate via RIE where the developed resist is used as a mask. The RIE chemistry has to be carefully selected to enhance anisotropic etching of the $\mathrm{Si}$ without etching the mask material, as isotropic etching leads to undesired broadening of patterns, see Figure 5-16. To achieve anisotropic etching, a variety of processes have been developed and can be found in the literature. After rigorous iterations of different RIE chemistry we have outlined the following two chemistries for the pattern transfer purpose, see Table 5-2.

(a)

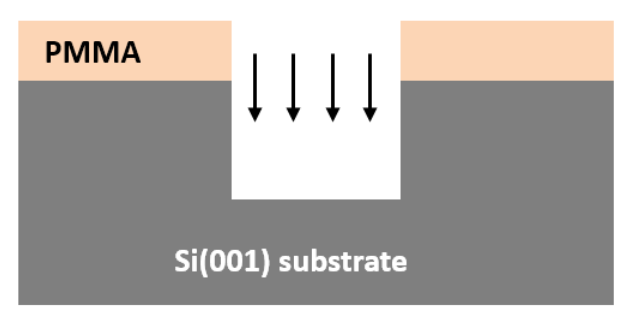

(b)

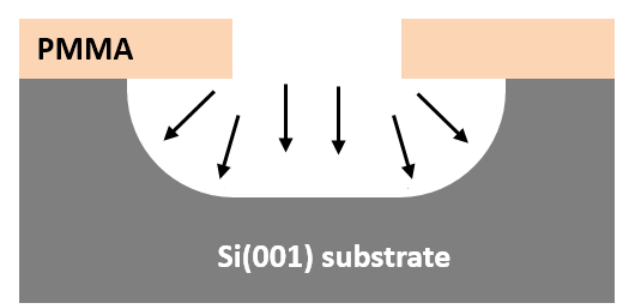

Figure 5-16: (a) anisotropic etching, and (b) isotropic etching of Si during RIE.

Table 5-2: Recipe used for Si etch during RIE

\begin{tabular}{|c|c|c|c|c|c|c|c|c|}
\hline $\mathbf{S} / \mathbf{N}$ & $\begin{array}{c}\mathbf{O}_{2} \\
(\mathbf{s c c m})\end{array}$ & $\begin{array}{c}\mathbf{C H F}_{3} \\
(\mathbf{s c c m})\end{array}$ & $\begin{array}{c}\mathbf{S F}_{\mathbf{6}} \\
(\mathbf{s c c m})\end{array}$ & $\begin{array}{c}\mathbf{C}_{\mathbf{4}} \mathbf{F}_{\mathbf{8}} \\
(\mathbf{s c c m})\end{array}$ & $\begin{array}{c}\text { Press. } \\
(\mathbf{m T o r r})\end{array}$ & $\begin{array}{c}\text { RF- } \\
\text { Power } \\
(\text { Watt })\end{array}$ & $\begin{array}{c}\text { Temp. } \\
\left({ }^{\circ} \mathbf{C}\right)\end{array}$ & $\begin{array}{c}\text { Etch Rate } \\
(\mathbf{n m} / \mathbf{m i n})\end{array}$ \\
\hline 1 & 5 & 40 & 2 & - & 20 & 25 & 27 & $\sim 8-10$ \\
\hline 2 & - & - & 12 & 27 & 10 & 30 & 10 & $\sim 15-20$ \\
\hline
\end{tabular}

The first recipe includes $\mathrm{O}_{2}$ in the chemistry therefore has a lower selectivity i.e, the etch rate of $\mathrm{Si}$ is only slightly higher than PMMA resist (oxygen plasma etches hydrocarbons). The final depth of the pit for this recipe is strongly limited by the thickness of the PMMA 
itself. However, at the end of the cycle the pits are smoother with minimal or no PMMA remaining on the $\mathrm{Si}$. The second recipe is a derivative of Bosch etch process. The selectivity of this recipe to etch $\mathrm{Si}$ is 1.5 times more than the etch rate of PMMA resulting in more controlled etch rates with smooth sidewalls. The Oxford Plasmalab 100 RIE manual specifies that the second recipe combined with inductively coupled plasma (ICP) can yield submicron Si etch with etch rates of $>130 \mathrm{~nm} / \mathrm{min}$, see Figure 5-17. For our current work, we did not use ICP during RIE, so as to reduce the etch rates since only shallow (10-20 $\mathrm{nm})$ pit depths were desired.

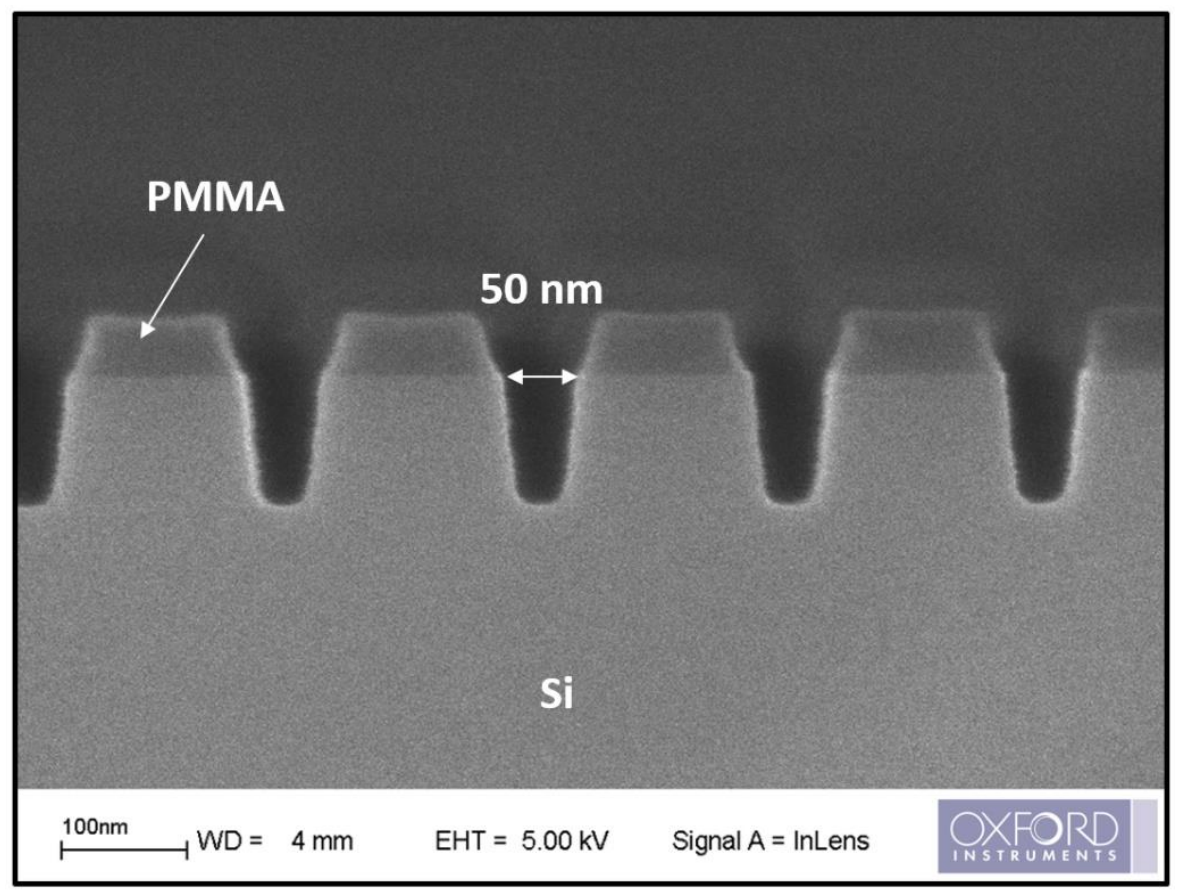

Figure 5-17: cross-sectional SEM image of $50 \mathrm{~nm}$ wide features etched with $\mathrm{SF}_{6}(12 \mathrm{sccm})$ and $\mathrm{C}_{4} \mathrm{~F}_{8}(27 \mathrm{sccm})$ chemistry at $10{ }^{\circ} \mathrm{C}, 10$ mTorr pressure, $750 \mathrm{~W}$ ICP power and $20 \mathrm{~W}$ RF power. (source: Oxford PlasmaLab 100 RIE manual)

For both RIE chemistries it was found that increasing the $\mathrm{SF}_{6}$ increases the Si etch rate, however the effect is isotropic resulting in wide and non-uniform pits. Addition of $\mathrm{CHF}_{3}$ or $\mathrm{C}_{4} \mathrm{~F}_{8}$ enhances the anisotropy during etch. A more detailed theory regarding the effects 
of mixing various chemistry for $\mathrm{Si}$ etch is presented in the literature. ${ }^{127}$ For our current work, we adopted $\mathrm{SF}_{6}+\mathrm{C}_{4} \mathrm{~F}_{8}$ chemistry with $\sim 1$ min of etch time yielding pit depths of 10$20 \mathrm{~nm}$.

Post RIE, the residual PMMA resist removal is crucial as any residue will impede the epitaxial growth during the deposition in MBE. As PMMA resist is simply a polymer (hydrocarbon) there are multiple ways to get rid of it. We tested the following 3 recipes and all were found to be effective and successful in removing the residual resist from $\mathrm{Si}$.

1. $10 \mathrm{~min}$ in acetone sonicator followed by $15-20 \mathrm{~min}$ in March Plasma cleaner with 100 W of RF power.

2. $10 \mathrm{~min}$ in acetone sonicator followed by $45-60 \mathrm{~min}$ in UV-ozone lamp box.

3. $10 \mathrm{~min}$ in acetone sonicator followed by $7-10 \mathrm{~min}$ in IMEC solution. (IMEC is a mixture of sulphuric acid and peroxide in 2:1 ratio)

To be consistent in our experimental approach, all of our samples followed recipe- 1 for the removal of residual PMMA resist. Figure 5-18 shows the AFM image comparing the line scans before and after the RIE/ resist removal stage.
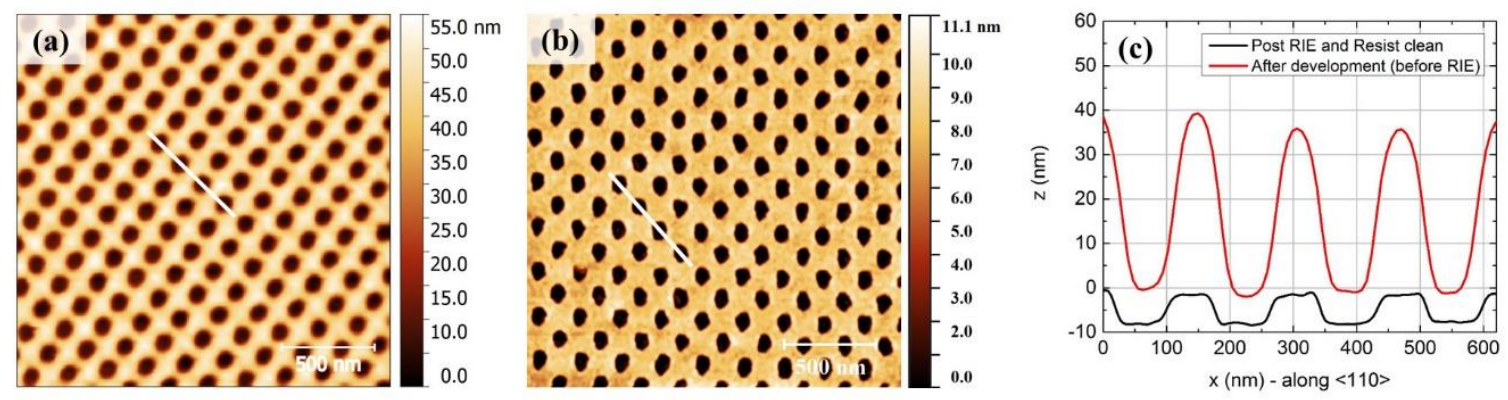

Figure 5-18: $2 \times 2 \mu \mathrm{m}^{2}$ AFM images showing $150 \mathrm{~nm}$ pattern pitch; (a) after the development stage (before RIE), (b) post RIE and resist removal stage, and (c) line scan along $\langle 110\rangle$ comparing the pattern morphology showing square-wave like patterns post RIE. 


\subsection{Summary}

For successful patterning of QDs the quality of 2D template plays a vital role. In this chapter, we mainly discussed the shortcomings of our FIB based patterning approach and discussed in more detail about our EBL based patterning approach to create highly ordered and uniform arrays of templated Si substrates. A summary of our optimized parameter for the EBL based patterning approach is presented in Table 5-3.

Table 5-3: Optimized process variables for EBL based patterning approach.

\begin{tabular}{|c|l|c|}
\hline S/N & \multicolumn{1}{|c|}{ Parameter } & Remarks \\
\hline 1 & Resist material & 950K PMMA-A2 \\
\hline 2 & Resist thickness & $50-70 \mathrm{~nm}$ \\
\hline 3 & Beam/ Exposure energy & Pattern pitch dependent \\
\hline 4 & Exposure (dose) & $(1: 3)$ MIBK:IPA \\
\hline 5 & Developer solution & $7-10$ sec at RT \\
\hline 6 & Development time and temp. & Etch for 1 min resulting in 10-20 nm deep pits \\
\hline 7 & RIE $\left(\mathrm{SF}_{6}+\mathrm{C}_{4} \mathrm{~F}_{8}\right)$ &
\end{tabular}

EBL based patterning approach involves RIE which basically etches the Si anisotrpically through the resist window/mask, ultimately creating pits with flat bottom i.e., square-wavelike pattern. Transforming such patterned surface morphology to a smooth and pseudosinusoidal surface for QD growth requires a systematic study and understanding of the effects of substrate cleaning process, Si buffer layer growth conditions and annealing process. More details on the processing and growth on these patterned substrates will be discussed in the following chapter. 


\section{Chapter 6: Growth of $\mathrm{Si}_{1-\mathrm{x}} \mathrm{Ge}_{\mathrm{x}}$ QDs on a patterned $\mathrm{Si}(001)$ substrate}

In this chapter, we present our comprehensive investigation on the directed self-assembly of Si-Ge alloy QDs on patterned Si surfaces with variable morphology. This includes the effects of Si buffer layer growth on substrate pattern morphology along with the effects of critical growth parameters like annealing time, growth temperature, pattern orientation, pitch and alloy thickness during QD evolution. It is important to understand the selfassembly process of QDs on such patterned substrates to yield highly ordered and morphologically uniform array of QDs. Therefore, we present here a very simple growth kinetic model based on our experimental data to describe the evolution of QDs on such patterned substrate. Further, we present our results for the investigation of QD siteselection on patterned $\mathrm{Si}(001)$ substrates as a function of underlying substrate pattern morphology and compare it to existing experiments and modeling.

\subsection{Introduction}

QD formation requires growth conditions where adatom diffusion lengths match or exceed the intrinsic lengthscale, which can be expressed either as a critical nucleus size ${ }^{34,35}$, or in terms of the instability wavelength, $\lambda_{\text {ATGS. }}{ }^{33}$ This "wavelength" arises due to the competition between the reduction of the elastic energy by 3D island formation vs. the increase in surface energy; see Chapter 1 for more details on the evolution of these 3D island evolution. When the artificially imposed pattern periodicity $\left(\lambda_{\mathrm{p}}\right)$ becomes comparable to the $\lambda_{\text {ATGS, }}$, the interaction between these two wavelengths becomes important to QD site selection. ${ }^{39}$ So far the interaction between $\lambda_{\mathrm{ATGS}}$ and $\lambda_{\mathrm{p}}$, has not been systematically explored. Here we present results of a detailed experimental study on QD site selection for $\mathrm{Si}_{0.5} \mathrm{Ge}_{0.5}$ alloys. We show that when the pattern evolves, at fixed $\lambda_{\mathrm{p}}$, from 
a discrete "pit-in-terrace" morphology to a quasi-sinusoidal shape, a deterministic transition occurs in the site selection, without coarsening. The occurrence of this transition depends sensitively on $\lambda_{\mathrm{p}}$, annealing time ( $\left.\mathrm{t}_{\text {anneal }}\right)$, pattern orientation and $\mathrm{Si}_{0.5} \mathrm{Ge}_{0.5}$ alloy thickness.

\subsection{Growth/ Experiment}

After the patterning of $\mathrm{Si}(001)$ substrates via EBL, the substrate is chemically cleaned ex situ prior to the deposition in $\mathrm{MBE}$, see the low temperature cleaning process in Chapter 2 for more details. The H-terminated $\mathrm{Si}(001)$ surface was then desorbed in situ at $550{ }^{\circ} \mathrm{C}$ after an overnight temperature ramp, plus $5 \mathrm{hr}$. prebake at $400{ }^{\circ} \mathrm{C}$. The substrate was then cooled to $450{ }^{\circ} \mathrm{C}$ for the deposition of a $50 \mathrm{~nm}$ thick $\mathrm{Si}$ buffer layer followed by the deposition of $\mathrm{Si}_{0.5} \mathrm{Ge}_{0.5}$ alloy film. We followed an unusual protocol for the QD selfassembly process. This turned out to provide crucial control over the size and morphology of the QDs. The Si-Ge alloy layer was first deposited at $450{ }^{\circ} \mathrm{C}$ to ensure a nominally conformal surface profile. The QDs were then formed during a post-deposition in situ

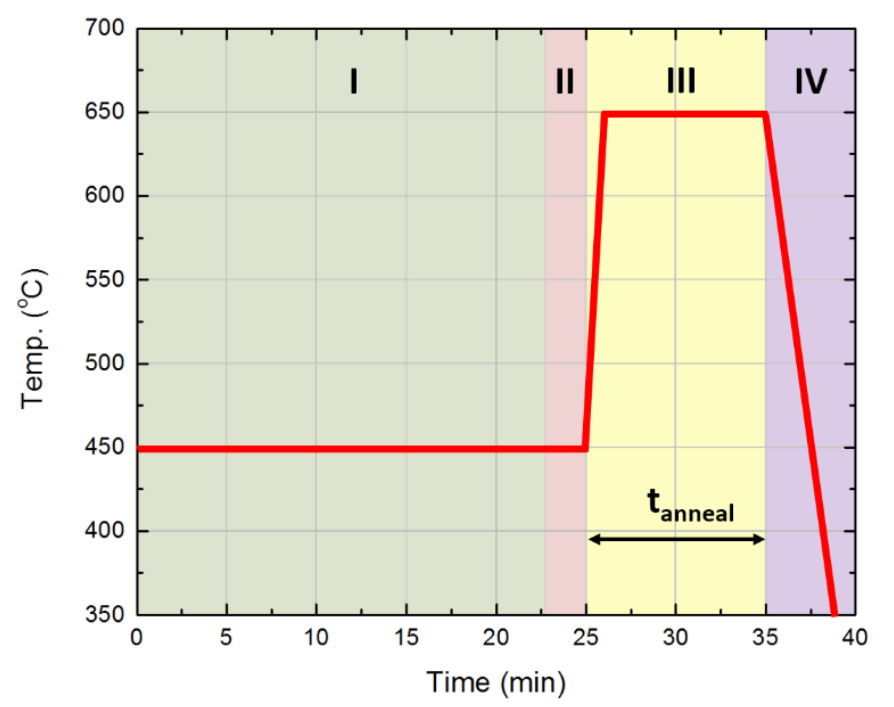

Region I: Deposit $50 \mathrm{~nm}$ Si buffer layer

Region II: Deposit $\mathrm{Si}_{0.5} \mathrm{Ge}_{0.5}$ alloy layer

Region III: Anneal the sample at $650^{\circ} \mathrm{C}$

Region IV: Quench

Figure 6-1: Time vs growth temperature plot of the deposition cycle in MBE divided into 4 distinct regions. $t_{\text {anneal }}$ is the annealing time. 
anneal at $650{ }^{\circ} \mathrm{C}$ for $5 \mathrm{~min}\left(\mathrm{t}_{\text {anneal }}\right)$. The low temperature buffer deposition and the post anneal process of forming the QDs was very critical as ramping the temperature during buffer deposition resulted in shallow pits with facets or a complete loss of patterns. A schematic diagram representing the entire growth process in terms of temperature and time is shown in Figure 6-1.

We fabricated two major pattern fields on the Si substrate, dose variation test field and constant dose test field, as discussed in previous chapter where the pattern periodicity range was $75-200 \mathrm{~nm}$, see Figure 5-9 in previous chapter. Similar patterns were processed in multiple wafers to ensure repeatability of the patterning and growth process discussed here. Next, we will present the results of Si buffer layer growth on these EBL patterned region.

\subsection{Si buffer growth: Homoepitaxy}

For successful growth of QDs with good epitaxy, the growth surface should be free from impurities and is required to be planar without any clusters (roughness). Therefore, a thin epitaxial layer of Si buffer layer is deposited on $\mathrm{Si}(001)$ so that the growth surface is smooth with continuous transition from substrate to epitaxial layer which is free from impurities,
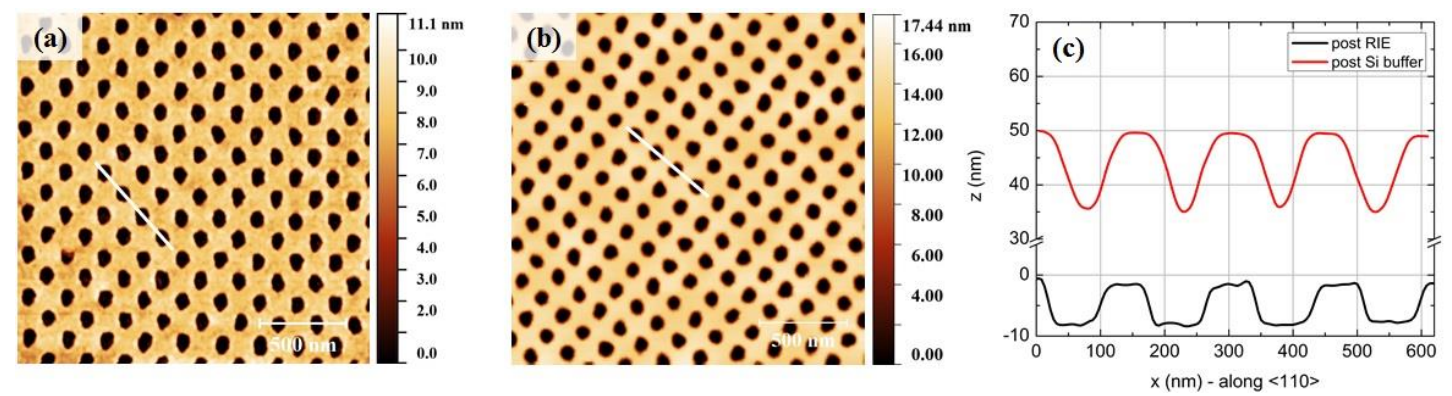

Figure 6-2: $2 \times 2 \mu \mathrm{m}^{2} \mathrm{AFM}$ images of $150 \mathrm{~nm}$ pitch pattern: (a) after RIE and resist cleaning, (b) after $50 \mathrm{~nm}$ of $\mathrm{Si}$ buffer growth at $450{ }^{\circ} \mathrm{C}$, and (c) line scan along $<110>$ showing the transition of rough and square-wave-like pattern to smooth Gaussian-like pit pattern profile. 
disorder and misalignments present at the interface. Apart from this, the Si buffer layer plays a critical role for our patterned substrates as it results in the smoothening of rough and damaged surface due to RIE etching, see Figure 6-2.
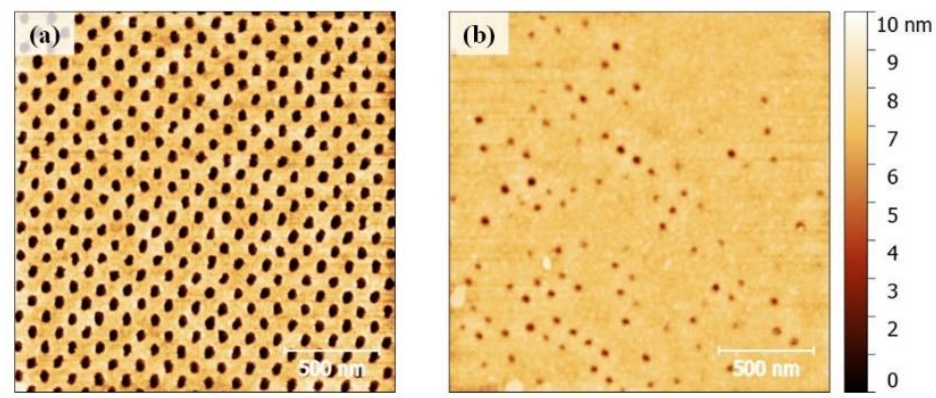

Figure 6-3: $2 \times 2 \mu \mathrm{m}^{2} \mathrm{AFM}$ images of $100 \mathrm{~nm}$ pitch pattern (a) after RIE, and (b) after 50 $\mathrm{nm}$ Si buffer deposited at $550^{\circ} \mathrm{C}$.

In order to obtain an ideal pattern for the growth of QDs, optimal buffer thickness and growth temperature are crucial to defining the final pattern morphology. For instance, a Si buffer layer deposited at higher temperature or beyond the ideal thickness can result in complete smoothening and loss of the patterns, see Figure 6-3. This effect was observed to be very pitch-sensitive - patterns with finer pitch were observed to disappear first. Presumably this occurs when the surface diffusion length is comparable to the pitch. For

(a) $\lambda=100 \mathrm{~nm}$

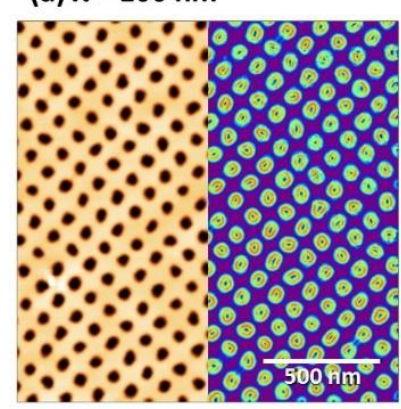

(b) $\lambda=150 \mathrm{~nm}$

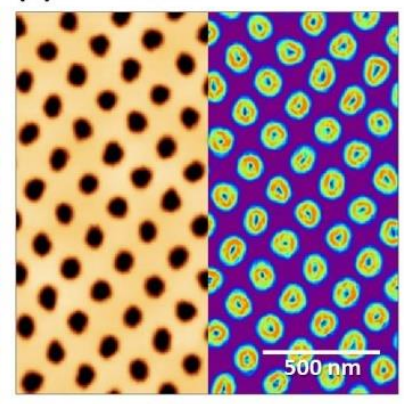

(c) $\lambda=200 \mathrm{~nm}$

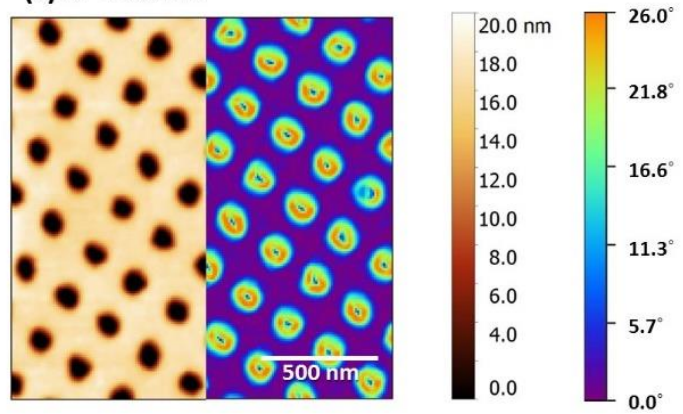

Figure 6-4: AFM images along with the local-area slope image after $50 \mathrm{~nm}$ of Si buffer deposition for pattern with pitch (a) $100 \mathrm{~nm}$, (b) $150 \mathrm{~nm}$, and (c) $200 \mathrm{~nm}$. 
our experiments, we deposited $50 \mathrm{~nm}$ of $\mathrm{Si}$ buffer at $450{ }^{\circ} \mathrm{C}$ where the patterns with pitch $\geq 100 \mathrm{~nm}$ were preserved. For patterns with pitch less than $100 \mathrm{~nm}$, the $50 \mathrm{~nm}$ thick buffer deposited at $450{ }^{\circ} \mathrm{C}$ generally resulted in shallower pits or loss of pattern. Therefore, further optimization work for buffer layer growth will be required for finer-pitch patterns, likely requiring smaller thickness and lower growth temperature.

The peak-to-valley depths measured for patterns after Si buffer growth was in the range of $\sim 15-20 \mathrm{~nm}$ with pit-sidewall slopes of $15-26^{\circ}$. The morphological evolution of pits after buffer deposition was observed to be less sensitive to the pattern pitch, see Figure 6-4. The size of the pit opening (W), in combination with Si buffer layer growth, permits variation of the pattern morphology. However, for pits with very small opening width ( $\mathrm{W}<50-60$ $\mathrm{nm})$ the buffer layer results in shallow pits $(<10 \mathrm{~nm})$ with sidewall slope of $5-11^{\circ}$ or a complete smoothening, see Figure 6-5. Similar observation of the influence of Si buffer layer on pit-patterns has been well reported in the literature, ${ }^{105,128}$ where pit sidewalls are composed of $\{113\}$ facets while the corners are decorated by $\{103\}$ facets along with shallower $\{11 \mathrm{n}\}$ facets at pit bottoms and edges for larger pitch pattern $\left(\lambda_{\mathrm{p}}>400 \mathrm{~nm}\right)$.
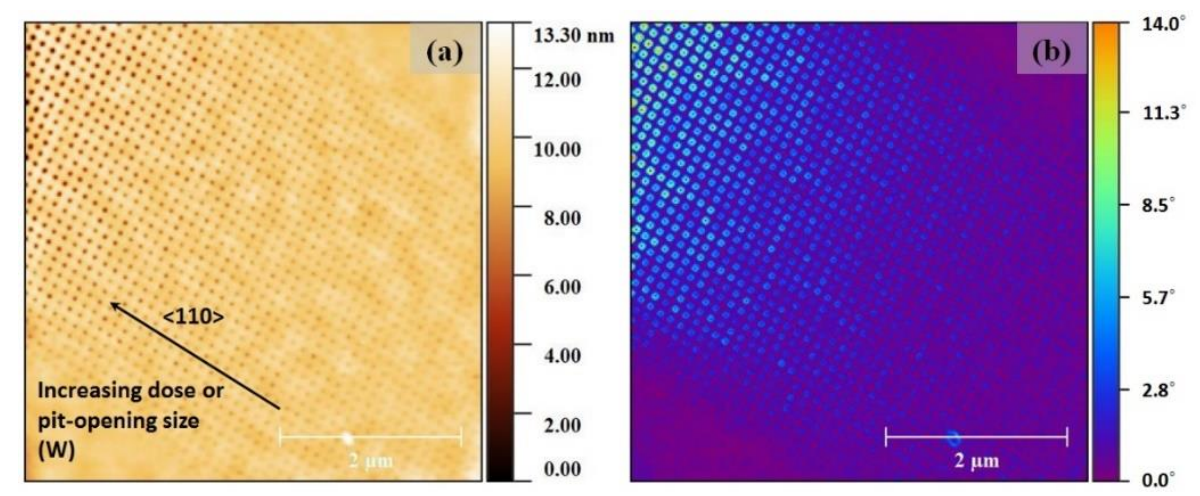

Figure 6-5: (a) $5 \times 5 \mu \mathrm{m}^{2}$ AFM image showing the disappearance of pits at the lowest dose region where the pits have narrow opening and shallow depth, (b) local slope image of the same AFM image showing the decreasing pit-sidewall slope with decreasing dose. 


\subsection{Pattern morphology: pit-in-terrace pattern vs quasi-sinusoidal}

As discussed in the previous chapter, the patterns used here are dot patterns, where only a "dot-pixel" is exposed to create a pit during etching. The pit opening width, W, scales with the electron dose at the dot-pixel; however, there is a critical dose beyond which adjacent

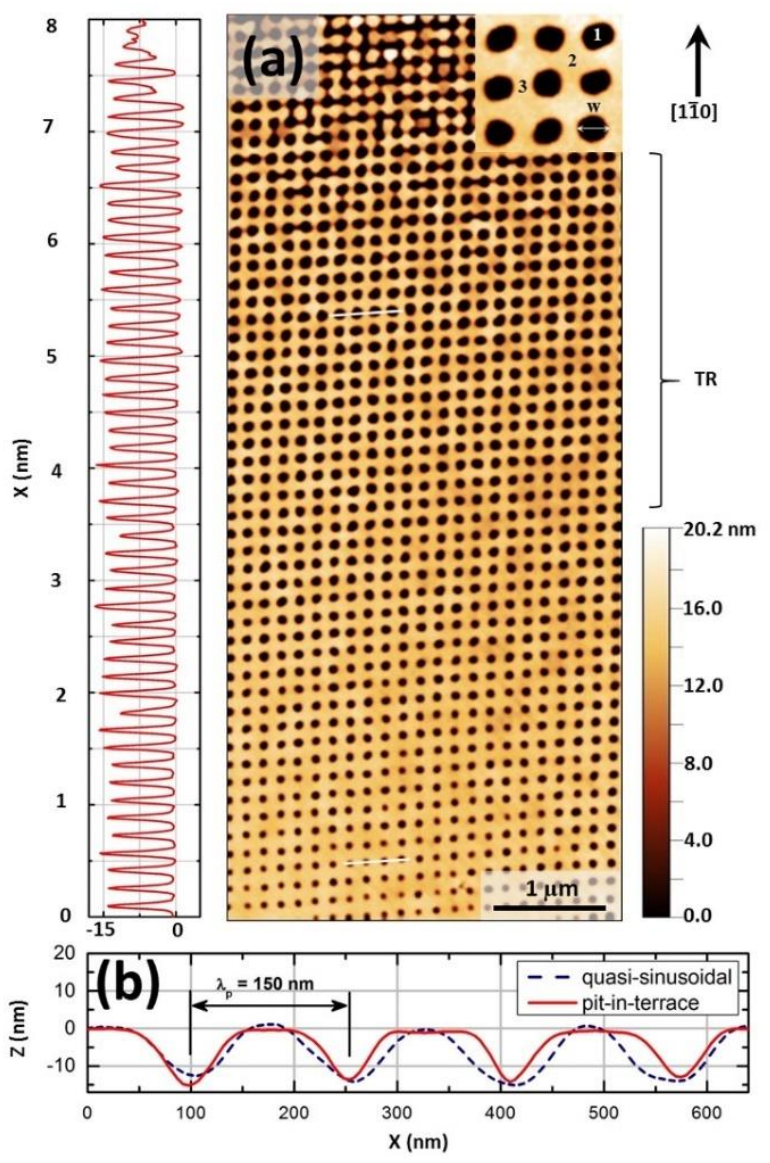

Figure 6-6: (a) Pattern with $\lambda_{\mathrm{p}}=150 \mathrm{~nm}$ after $\mathrm{Si}$ buffer growth. The long linescan along [11̄0] reveals the gradual evolution from pit-in-terrace to quasi-sinusoidal. The inset defines the locations: (1) pit bottom, (2) crown region and (3) saddle region. (b) Line scan along [110] comparing pit-in-terrace vs sinusoidal-like profile.

pits overlap, degrading the pattern quality. For the dose-variation pattern test fields, we varied the electron dose continuously along [1필 while retaining fixed pitch. Figure 6-6 shows the effect of dose variation where $\mathrm{W}$ is increasing along [1 10$]$. For all the samples 
here, the pit depth was held constant by fixing the RIE etch time, allowing the patterns to be characterized by $\mathrm{W}$ and $\lambda_{\mathrm{p}}$.

Small values of $\mathrm{W} / \lambda_{\mathrm{p}}$ have a pit-in-terrace profile where discrete pits are surrounded by flat (001) terraces. Larger values of $\mathrm{W} / \lambda_{\mathrm{p}}$ have a quasi-sinusoid profile as shown in Figure 6-6(b). To differentiate the two types of profile, we define quasi-sinusoidal profiles to occur when $W / \lambda_{p} \geq 2 / 3$. Patterns with $W / \lambda_{p}<2 / 3$ will be considered as pit-in-terrace. We also define a "transition region" (TR) where adjacent sites significantly overlap, eventually leading to pattern disintegration at larger values of $\mathrm{W} / \lambda_{\mathrm{p}}$, see the line scan along $[1 \overline{1} 0]$ in Figure 6-6. Growth on such varying pattern morphology is discussed in Section 6.9.

\subsection{Annealing time and growth temperature: Growth kinetics}

As discussed earlier, the $\mathrm{Si}_{0.5} \mathrm{Ge}_{0.5}$ alloy layer was deposited at $450{ }^{\circ} \mathrm{C}$. The alloy layer was observed to be nominally conformal with the substrate profile due to the low deposition temperature where surface diffusion of adatoms is restricted. To form the QDs, the surface diffusion length of adatoms should match or exceed the intrinsic lengthscale of the material being deposited $\left(\lambda_{\mathrm{ATGS}}\right.$ for $\mathrm{Si}_{0.5} \mathrm{Ge}_{0.5} \sim 75 \mathrm{~nm}$ ). For our conditions, a minimum $650{ }^{\circ} \mathrm{C}$ annealing temperature was required to initiate the self-assembly of QDs, where the estimated surface diffusion length $\left(\mathrm{d}_{1}\right)$ of adatoms was $\sim 90 \mathrm{~nm}$. The estimation of the surface diffusion length is discussed in Section 6.8. See Figure 6-1 for the annealing process, highlighting the anneal temperature and time. In this section, for a constant $\mathrm{Si}_{0.5} \mathrm{Ge}_{0.5}$ alloy thickness of $1.3 \mathrm{~nm}$ deposited at $450{ }^{\circ} \mathrm{C}$, we will present the results for the formation of QDs at $650{ }^{\circ} \mathrm{C}$ with annealing time ( $\left.\mathrm{t}_{\text {anneal }}\right)$ as the variable. Note that in all cases, for thickness $\mathrm{h}=1.3 \mathrm{~nm}$, the QDs formed in the subsequent anneal present good 
$\{105\}$ facets, typical of the Si-Ge system. This is important, since faceting tends to be an indicator that the growth surface after patterning is not grossly contaminated or defective.

Results for the growth of $\mathrm{Si}_{0.5} \mathrm{Ge}_{0.5}$ QDs on constant dose pattern fields with various pitches are presented in Figure 6-7, showing AFM topography scans along with line scans comparing the results for varying the annealing time. The scans were compiled from three different samples where $t_{\text {anneal }}$ was varied as $2 \min 30 \mathrm{sec}, 5 \mathrm{~min}$ and $10 \mathrm{~min}$ as shown. The three AFM scans for each $\lambda_{\mathrm{p}}$ comparing the results of varying $\mathrm{t}_{\text {anneal }}$ were taken from a constant dose region of $2 \times 10^{-3} \mathrm{pC}, 2.5 \times 10^{-3} \mathrm{pC}$ and $4.5 \times 10^{-3} \mathrm{pC}$ for $\lambda_{\mathrm{p}}=100 \mathrm{~nm}, 150 \mathrm{~nm}$ and $200 \mathrm{~nm}$ respectively for uniform comparison.

For $100 \mathrm{~nm}$ pitch, it is clearly observed in Figure 6-7(a) that increasing the annealing time results in the increase of QD size but at large $t_{\text {anneal }}(\geq 10 \mathrm{~min})$, Ostwald ripening of QDs is observed where bigger QDs are formed at the expense of smaller ones. Similarly, QD size increases with $t_{\text {anneal }}$ for patterns with $\lambda_{p}=150 \mathrm{~nm}$ and $200 \mathrm{~nm}$, see Figure 6-7(b) and (c). However, with reference to the AFM images, Ostwald ripening diffusion between neighboring QDs at large $t_{\text {anneal }}(\geq 10 \mathrm{~min})$ was not as substantial as it was for the $100 \mathrm{~nm}$ pitch patterns. 
(a)

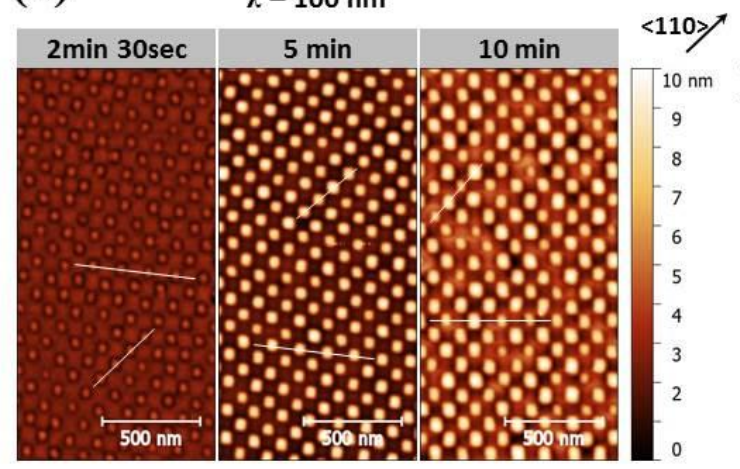

(b)

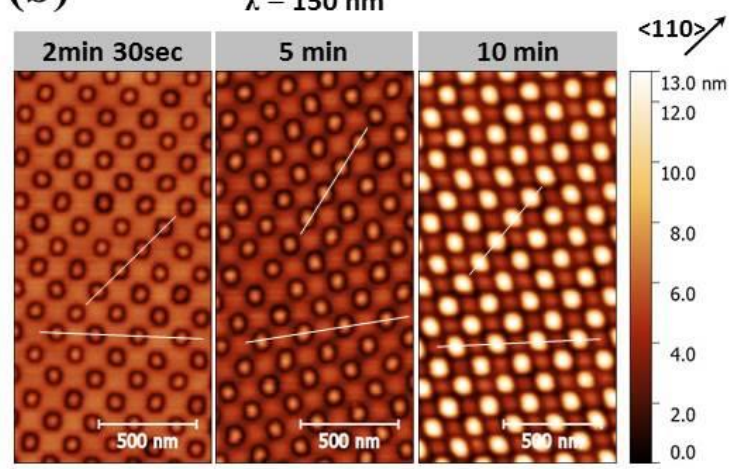

(c)

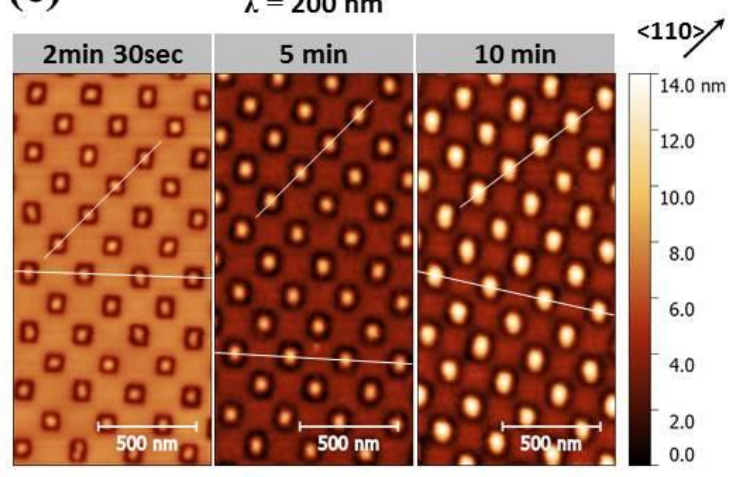

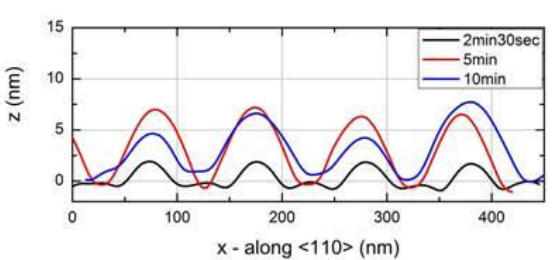
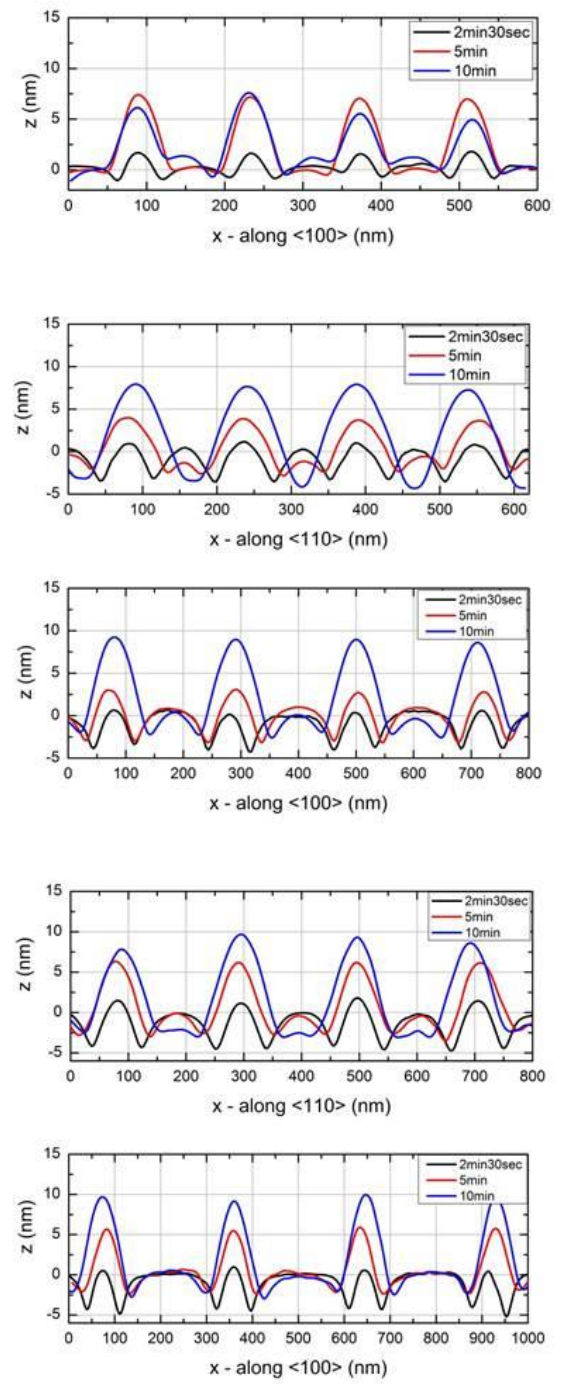

Figure 6-7: Comparison of the AFM images with their corresponding line scans along $\langle 110\rangle$ and $\langle 100\rangle$ for the deposition of $1.3 \mathrm{~nm}$ of $\mathrm{Si}_{0.5} \mathrm{Ge}_{0.5}$ on patterns with pitch (a) 100 $\mathrm{nm}$, (b) $150 \mathrm{~nm}$ and (c) $200 \mathrm{~nm}$. For patterns with same pitch, the scans were taken from three different samples varying $t_{a n n e a l}$, but in the same dose region such that the pit morphology is nominally identical prior to annealing. $\left(2 \times 10^{-3} \mathrm{pC}, 2.5 \times 10^{-3} \mathrm{pC}\right.$ and $4.5 \times 10^{-}$ $\left.{ }^{3} p C\right)$ 
To gain better insight into the growth kinetics, we performed a statistical study where volumetric analysis was performed for the AFM images shown in Figure 6-7. For volumetric analysis, we masked all the QDs within a $2 \times 2 \mu \mathrm{m}^{2}$ AFM image using the image processing software (gwyddion), such that 330, 150 and 80 QDs were selected for patterns with pitch of 100, 150 and $200 \mathrm{~nm}$, respectively. Each of the QDs were carefully masked making sure that the mask region would extend only up to the QD edge. The results for the QD volume distribution in absolute scale is given in Figure 6-8 (left side). Note that the volume derived from this method is only able to track and compare the QD volume above the local surface, i.e., the volume of material in the pit is not accounted for. Therefore, this will always underestimate the absolute value of QD volume. For the comparison of QD volume distribution at different $t_{\text {anneal }}$ for a specific $\lambda_{\mathrm{p}}$, the normalized distribution width

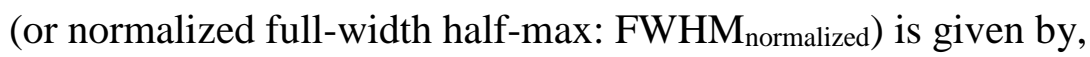

$$
\mathrm{FWHM}_{\text {normalized }}=2.355 * \sigma_{\mathrm{R}}
$$

In Equation 6-1, $\sigma_{\mathrm{R}}$ is the relative standard deviation (RSD), also known as coefficient of variation $(\mathrm{CV})$, is a standardized measure of dispersion of a probability distribution for comparison between two or more data sets. Here, $\sigma_{\mathrm{R}}$ is given by,

$$
\sigma_{\mathrm{R}}=\frac{\sigma}{<\mathrm{V}>}
$$

$\sigma$ is the standard deviation of the volume distribution and $\langle\mathrm{V}\rangle$ is the mean volume of the data. Note that the calculations here are based on a Gaussian or normalized distribution. The assumption of a normalized distribution follows from the fact that all of our volume distribution data satisfies the normalized distribution condition in both the probability plot 
and the Q-Q plot of statistics, see Appendix 4. The statistical data of the volume distribution curves in both absolute and normalized scale is given in Table 6-1.

Table 6-1: Statistical data for the distribution width, in absolute and normalized units, of probability density plots shown in Figure 6-8.

\begin{tabular}{|c|c|c|c|c|c|c|}
\hline $\begin{array}{l}\text { Pitch } \\
\left(\lambda_{p}\right)\end{array}$ & $\begin{array}{c}\text { Annealing } \\
\text { Time } \\
(\text { tanneal})\end{array}$ & $\begin{array}{c}\text { Mean } \\
\text { Volume }<\text { V }> \\
\mathbf{x 1 0}^{-23} \mathbf{m}^{3}\end{array}$ & $\begin{array}{c}\text { Std. } \\
\text { Deviation } \\
\text { (SD or } \sigma)\end{array}$ & $\begin{array}{l}\text { FWHM } \\
(2.355 \sigma)\end{array}$ & $\begin{array}{l}\text { Relative SD } \\
\text { (RSD or } \sigma_{R} \text { ) }\end{array}$ & $\begin{array}{c}\text { Normalized } \\
\text { FWHM } \\
(2.355 \sigma \mathrm{R})\end{array}$ \\
\hline \multirow{3}{*}{100} & $2 \mathrm{~min} 30 \mathrm{sec}$ & 0.296 & 0.061 & 0.144 & 0.206 & 0.486 \\
\hline & $5 \mathrm{~min}$ & 1.199 & 0.190 & 0.446 & 0.158 & 0.372 \\
\hline & $10 \mathrm{~min}$ & 1.790 & 0.765 & 1.802 & 0.428 & 1.007 \\
\hline \multirow{3}{*}{150} & $2 \mathrm{~min} 30 \mathrm{sec}$ & 0.903 & 0.120 & 0.283 & 0.133 & 0.314 \\
\hline & $5 \mathrm{~min}$ & 1.636 & 0.228 & 0.538 & 0.140 & 0.329 \\
\hline & $10 \mathrm{~min}$ & 3.221 & 0.242 & 0.570 & 0.075 & 0.177 \\
\hline \multirow{3}{*}{200} & $2 \min 30 \mathrm{sec}$ & 1.035 & 0.120 & 0.283 & 0.116 & 0.274 \\
\hline & $5 \mathrm{~min}$ & 2.879 & 0.321 & 0.756 & 0.112 & 0.263 \\
\hline & $10 \mathrm{~min}$ & 4.419 & 0.671 & 1.580 & 0.152 & 0.358 \\
\hline
\end{tabular}

The corresponding normalized probability density plots for QD volume distribution are given on the right side of Figure 6-8 where the abscissa is $\mathrm{V} /\langle\mathrm{V}\rangle$ and ordinate is $\mathrm{PDF} / \mathrm{PDF}_{\max }$. Here "PDF" is the normal probability density function given by,

$$
\mathrm{PDF}=\frac{1}{\sigma_{\mathrm{R}} \sqrt{2 \pi}} * \exp \left[\frac{-(\mathrm{x}-\mu)^{2}}{2 \sigma_{\mathrm{R}}^{2}}\right]
$$

where " $\mu$ " is the mean value of the distribution which is taken as 1 since the value of " $\sigma$ " has already been normalized by dividing with $\langle\mathrm{V}\rangle$. 
(a)
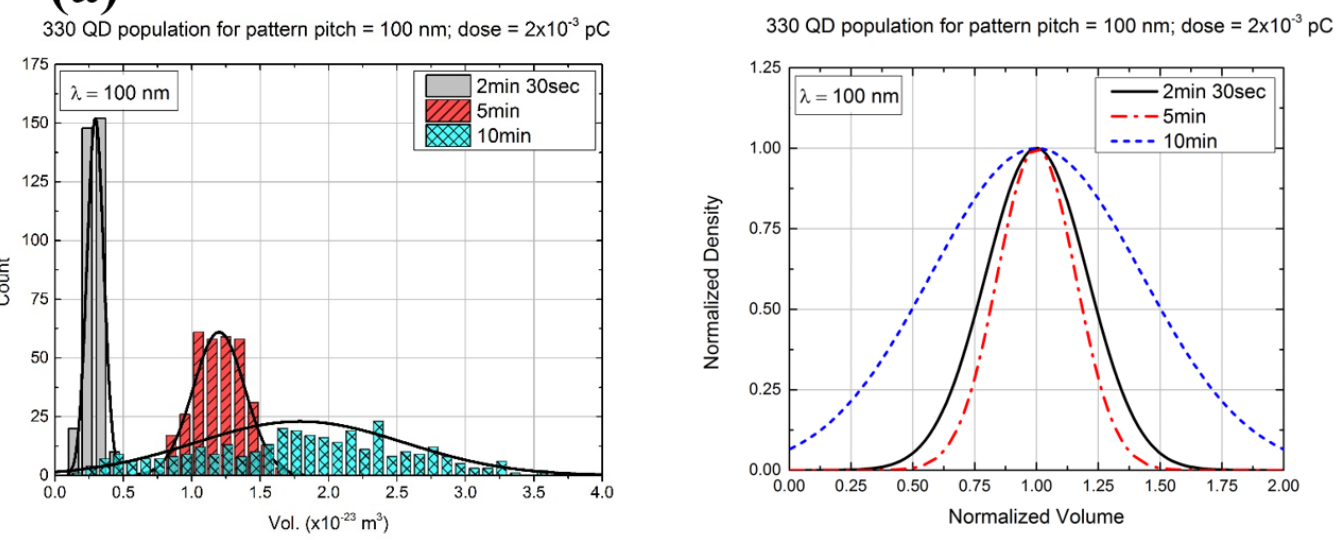

(b)
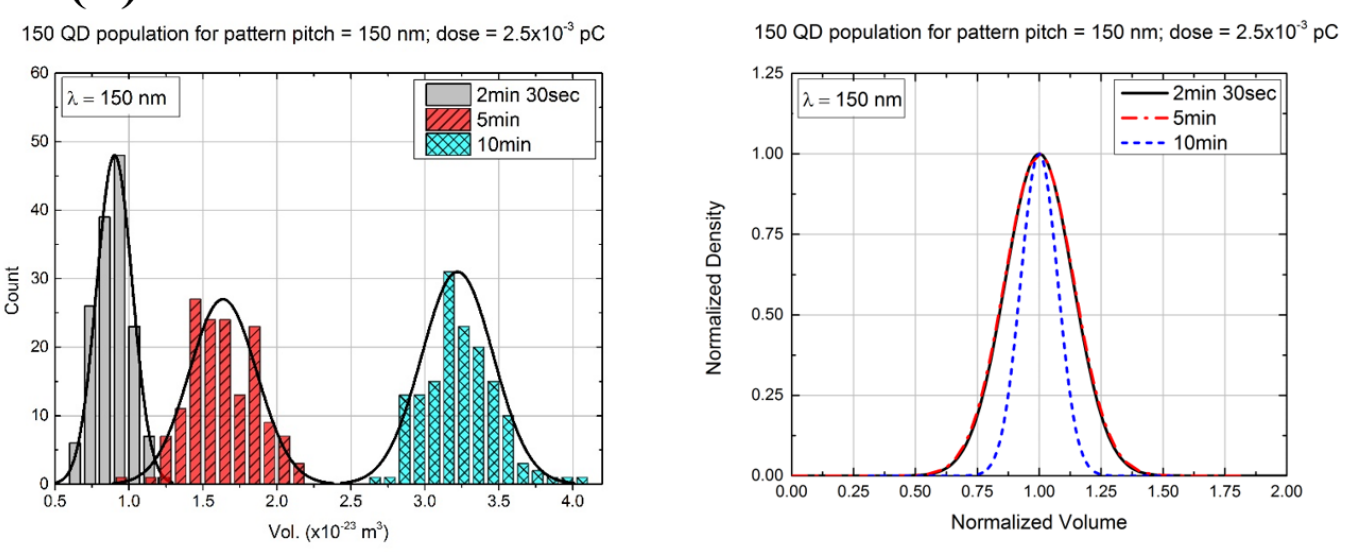

(c)
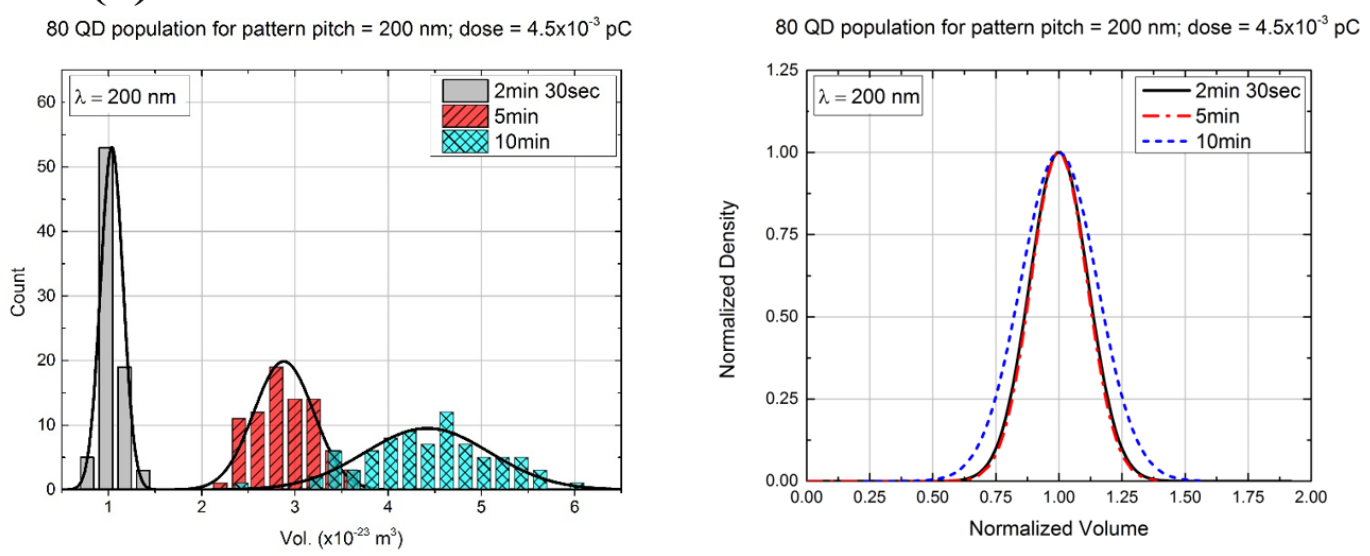

Figure 6-8: Comparison of volume distribution of QDs for the deposition of $1.3 \mathrm{~nm}$ of $\mathrm{Si}_{0.5} \mathrm{Ge}_{0.5}$ in absolute (left) and normalized (right) units, at various $\mathrm{t}_{\text {anneal }}$ for patterns with pitch (a) $100 \mathrm{~nm}$, (b) $150 \mathrm{~nm}$, and (c) 200nm. 
In Figure 6-8, the volume distribution plots on the left side clearly show for all the patterns that increasing the annealing time results in the increase of QD mean volume. Note that the deposited material for all these cases is fixed at $1.3 \mathrm{~nm}$ of $\mathrm{Si}_{0.5} \mathrm{Ge}_{0.5}$. Therefore, consumption of the wetting layer (WL) is the primary reason for the initial QD enlargement observed for all the cases here. Each pit acts as a material sink such that it collects material from the surroundings; the absolute collection area per unit pit is given by $\lambda_{\mathrm{p}}{ }^{2}$ (assuming no Ostwald ripening initially). However, the amount of material that each pit can collect is also determined by the kinetics such that the effective collection area is given by $\pi\left(\mathrm{d}_{1}\right)^{2}$, where $d_{l}$ is the surface diffusion length of the adatom species $(\sim 90 \mathrm{~nm})$, see Figure 6-9. Figure 6-10 compares the capture zone to the pitch for the specimens of interest here.

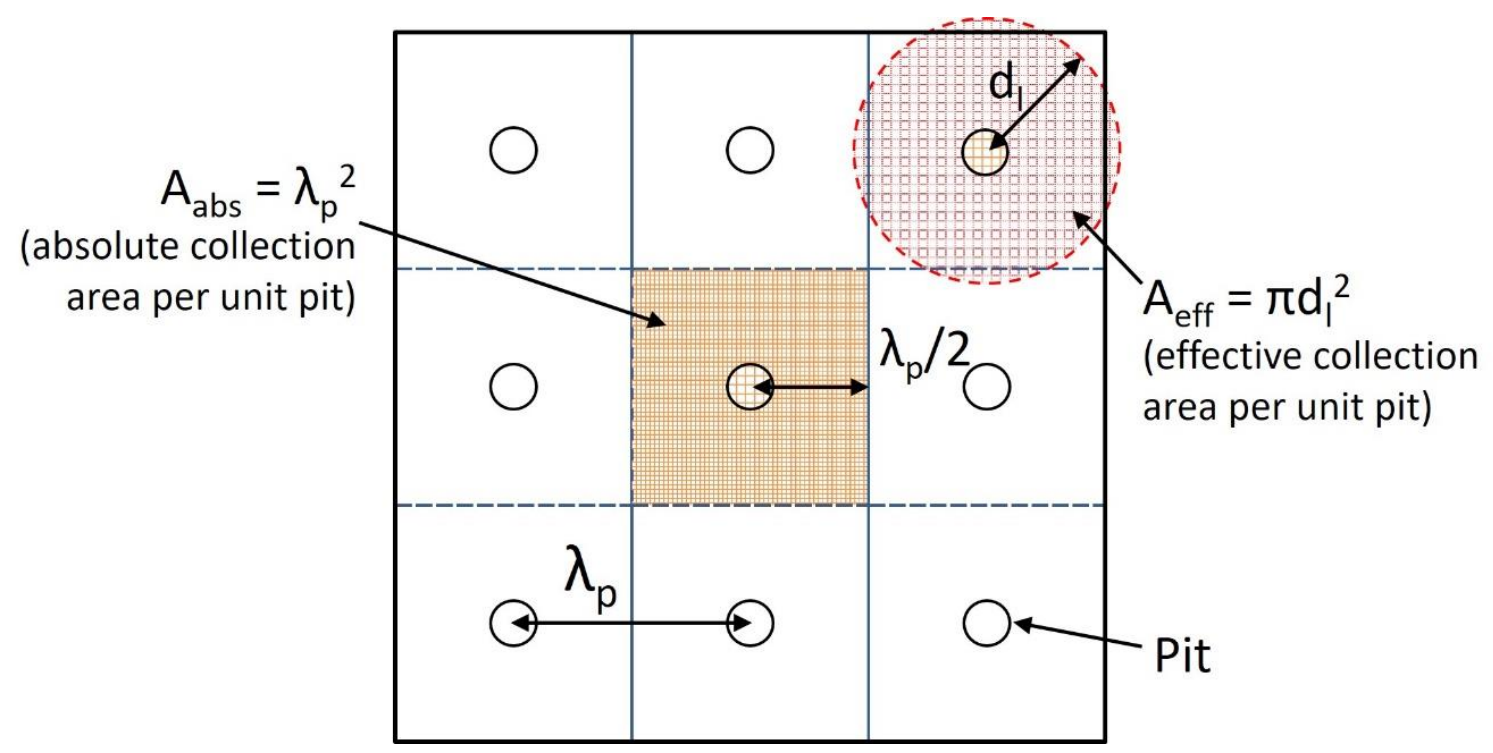

Figure 6-9: Schematic diagram of the absolute collection area and effective collection area per unit pit in terms of $\lambda_{p}$ and surface diffusion length $\left(d_{1}\right)$. If $d_{1}>\lambda_{p} / 2$ or comparable to $\lambda_{p}$, ripening of QDs will dominate as the annealing time progresses.

The normalized probability density plots on the right side of Figure 6-8 compare the distribution width at different $t_{\text {anneal }}$, where narrower distributions imply more uniform QD 
volume distributions. For $\lambda_{p}=100 \mathrm{~nm}$, the normalized volume distribution is comparatively narrow and similar for $t_{\text {anneal }} \leq 5$ min but significantly broadens at $t_{\text {anneal }}=10$ min, see the right side of Figure 6-8(a), and Table 6-1. For $\lambda_{\mathrm{p}}=150 \mathrm{~nm}$, the distribution width is fairly similar $\mathrm{t}_{\text {anneal }}=2.5$ and $5 \mathrm{~min}$, but the volume distribution width apparently narrows at $\mathrm{t}_{\text {anneal }}$ $=10 \mathrm{~min}$. For $\lambda_{\mathrm{p}}=200 \mathrm{~nm}$, the distribution width is similar for all the annealing time cases, see right side of Figure 6-8(c).

(a)

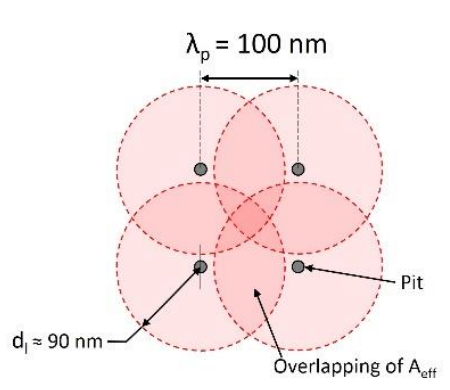

(b)

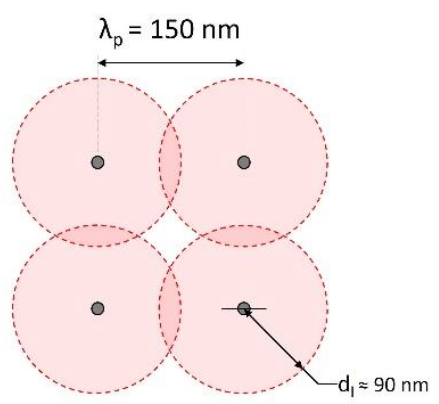

(c)

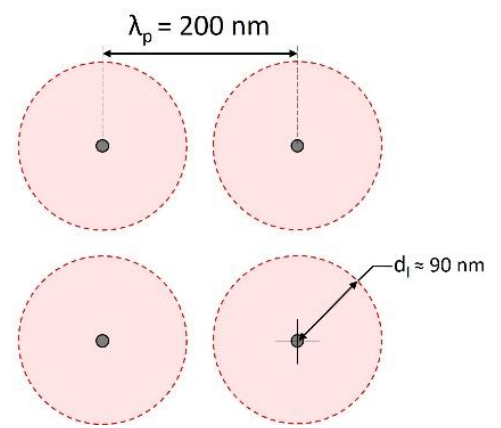

Figure 6-10: Effective collection area for four individual neighboring pits with pattern pitch of (a) $100 \mathrm{~nm}$, (b) $150 \mathrm{~nm}$, and (c) $200 \mathrm{~nm}$. The dimensions in the schematics are in scale for comparison where $d_{1}$ has been scaled to match $90 \mathrm{~nm}$ (refer section 6.8 for our diffusion length calculations)

Figure 6-11 shows the evolution of the mean volume and normalized FWHM for the three annealing times examined. Again, recall that the QD volumes measured here are only those above the surrounding (001) terrace. We do not observe any QDs forming below an annealing time of $1 \mathrm{~min}$ at $650{ }^{\circ} \mathrm{C}$, therefore at $\mathrm{t}_{\text {anneal }}=1 \mathrm{~min}$ we have taken $\langle\mathrm{V}\rangle=0$ for all the patterns. For all three pitches, up to $t_{\text {anneal }}=5 \mathrm{~min}$, the growth rate of QDs is nominally linear, and larger pitch leads to higher growth rate. This result is a bit surprising, since we would expect that if surface diffusion is rate limiting, the initial growth rate would be independent of pitch. Hence, we suggest that, at least for $\lambda_{\mathrm{p}}=100$ and $150 \mathrm{~nm}$, the mean 
QD growth rate, $\mathrm{G}=\mathrm{d}<\mathrm{V}>/ \mathrm{dt}$, depends only on the pattern-limited capture area, $\mathrm{A}_{\text {abs }}$ such that $\mathrm{G}=\lambda_{\mathrm{p}}{ }^{2}\left(\mathrm{dh} \mathrm{w}_{\mathrm{wl}} / \mathrm{dt}\right)$, where $\mathrm{h}_{\mathrm{wl}}$ is the wetting layer thickness. The linear dependence of volume vs. $t$ implies that $\mathrm{dh}_{\mathrm{w}} / \mathrm{dt}$ is constant, and the slope would be $\propto \lambda_{\mathrm{p}}^{2}$. The predicted ratio of slopes for $\lambda_{\mathrm{p}}{ }^{2}=200^{2}: 150^{2}: 100^{2} \mathrm{~nm}$ is $4: 2.25: 1$, while the measured ratios from Figure 6-11(a) are $\approx 3: 2: 1$. The agreement is reasonable, given the low data density in Figure 6-11(a). The poorer agreement, and more prominent non-linearity, for $\lambda_{\mathrm{p}}=200 \mathrm{~nm}$ indicates that this pitch is large enough to be in the diffusion-controlled regime. From the slopes of $\langle\mathrm{V}\rangle$ vs. t for $\lambda_{\mathrm{p}}=100$ and $150 \mathrm{~nm}$, we obtain a crude estimate of wetting layer consumption rate at $650{ }^{\circ} \mathrm{C}, \mathrm{dh}_{\mathrm{w}} / \mathrm{dt} \approx 0.24 \mathrm{~nm} / \mathrm{min}$. This regime of growth is labeled as Region-I in Figure 6-11(a).

The amount of WL volume available for each pit is $h_{w L} * \lambda_{p}{ }^{2}$. Figure 6-11(a) shows this maximum pattern-limited volume for each pitch as horizontal, color-coded lines. Note that as the QD volume approaches this limiting volume $\left(\mathrm{h}_{\mathrm{wL}} * \lambda_{\mathrm{p}}{ }^{2}\right)$ the saturation in the volume will be reached.

At larger anneal time ( $\left.\mathrm{t}_{\mathrm{anneal}}>5 \mathrm{~min}\right)$, for $\lambda_{\mathrm{p}}=100 \mathrm{~nm}$ at region II, the growth rate is limited as saturation value is reached $\left(1.3 \times 10^{-23} \mathrm{~m}^{3}\right)$, resulting in Ostwald ripening of the QDs. Thus, at $\lambda_{\mathrm{p}}=100 \mathrm{~nm}$ the growth rate is simply limited at larger $\mathrm{t}_{\text {anneal }}$ due to ripening where significant overlapping of $A_{\text {eff }}$ occurs between neighboring pits. For $\lambda_{\mathrm{p}}=150 \mathrm{~nm}$ at region III, the growth is close to linear throughout the anneal time (up to $10 \mathrm{~min}$ ) such that the saturation limit for the volume is just reached close to $\mathrm{t}_{\text {anneal }}=10 \mathrm{~min}$. The effect of ripening is observed to be suppressed as overlapping of $A_{\text {eff }}$ is minimal. For $\lambda_{\mathrm{p}}=200 \mathrm{~nm}$ at region IV, the growth rate is decaying; here, the surface diffusion length $\left(\mathrm{d}_{1}\right)<\lambda_{\mathrm{p}} / 2$ and the 
saturation limit for the volume $\left(5.2 \times 10^{-23} \mathrm{~m}^{3}\right)$ has not been reached since the flow of adatoms from flat regions in between the pits is kinetically restricted $\left(\mathrm{A}_{\mathrm{eff}}<\mathrm{A}_{\mathrm{abs}}\right)$.

Figure 6-11(b) shows the dependence of the normalized FWHM on time and $\lambda_{\mathrm{p}}$. Our interpretation of this graph for now is that the gray banded region represents the day-today variations in the pit-pattern itself (due to the EBL-based process). Hence only for the point $\lambda_{\mathrm{p}}=100 \mathrm{~nm}, \mathrm{t}_{\mathrm{anneal}}=10 \mathrm{~min}$ has Ostwald ripening clearly taken over. In the work by
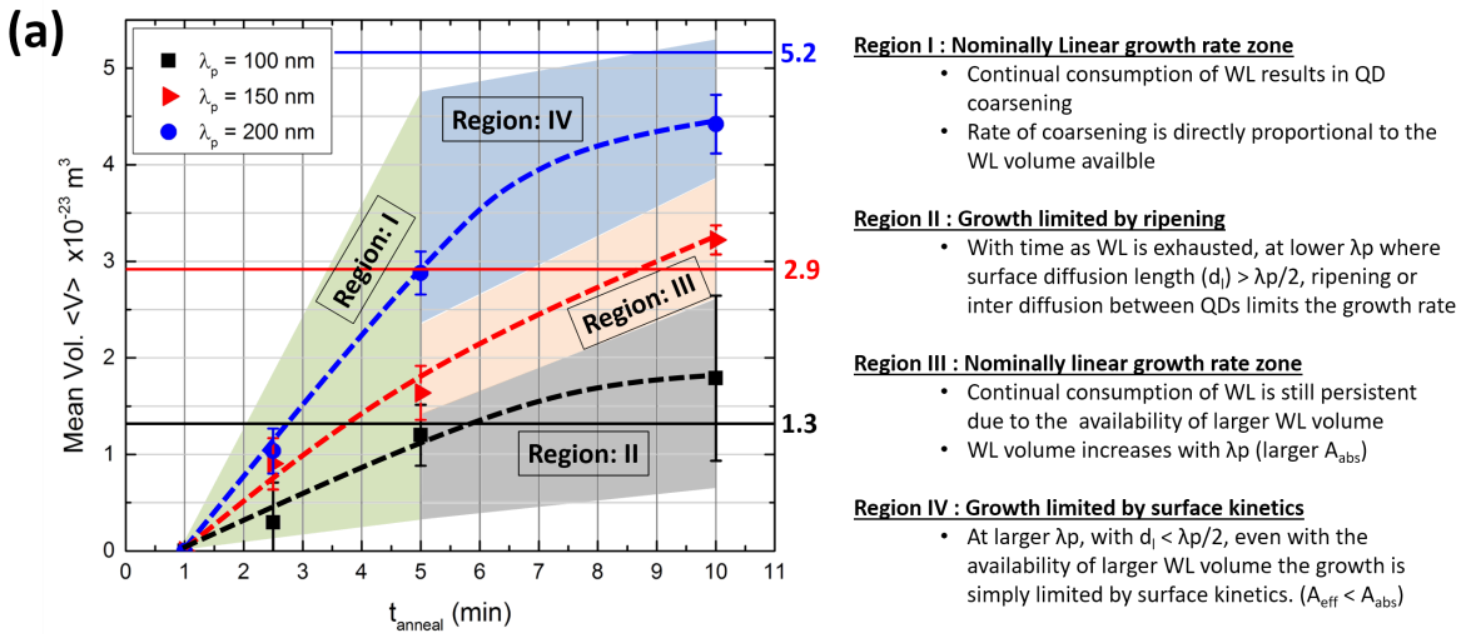

- Rate of coarsening is directly proportional to the WL volume availble

Region II : Growth limited by ripening

- With time as WL is exhausted, at lower $\lambda \mathrm{p}$ where surface diffusion length $\left(d_{1}\right)>\lambda p / 2$, ripening or inter diffusion between QDs limits the growth rate

Region III : Nominally linear growth rate zone

- Continual consumption of WL is still persistent due to the availability of larger WL volume

- WL volume increases with $\lambda p$ (larger $\left.A_{a b s}\right)$

Region IV : Growth limited by surface kinetics

- At larger $\lambda p$, with $d_{1}<\lambda p / 2$, even with the availability of larger WL volume the growth is simply limited by surface kinetics. $\left(A_{\text {eff }}<A_{\text {abs }}\right)$

(b)

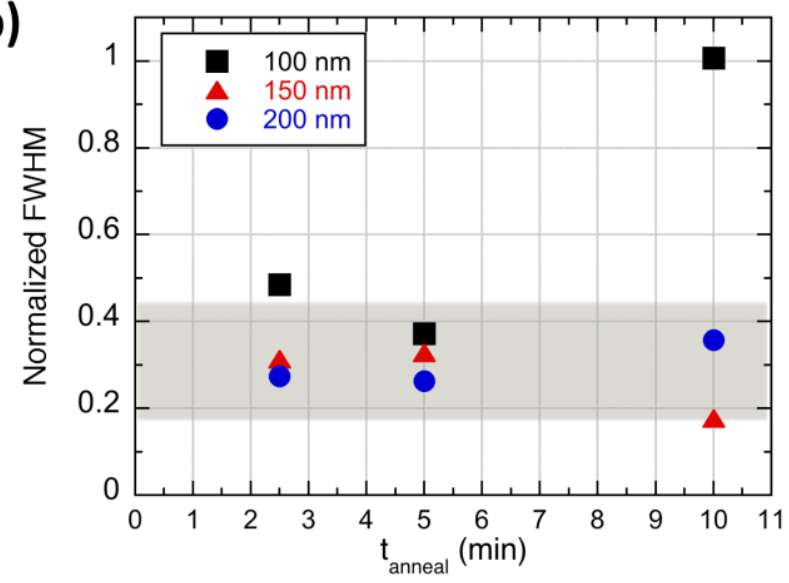

Figure 6-11: (a) Plot of mean volume vs anneal time at various pitch for $1.3 \mathrm{~nm}$ of $\mathrm{Si}_{0.5} \mathrm{Ge}_{0.5}$ alloy. The plot is subdivided into four distinct regions outlining the growth kinetics. (b) Plot of normalized FWHM vs anneal time 
Grydlik, et al., ${ }^{129}$ they show that their growth conditions lead to diffusion lengths of $>10$ $\mu \mathrm{m}$, encompassing of order hundreds of pits. Despite this, Ostwald ripening is not observed to occur. This is most easily understood if the patterned pits are perfect sinks for adatoms, so that interdot mass transfer is suppressed. In our experiments, the diffusion length is considerably shorter $(\approx 100 \mathrm{~nm})$, which only encompasses adjacent pit sites for the smallest pitches reported here. This much smaller diffusion length arises since we are using Si-Ge alloys (activation energies for Si diffusion are higher) and, more importantly, QDs are forming during an anneal rather than directly under a vapor flux. Growth by annealing requires that adatom detachment from steps occurs, increasing the overall activation barrier for QD growth. The sudden onset of ripening for $\lambda_{\mathrm{p}}=100 \mathrm{~nm}$ may be due to the fact that in this case, the QDs actually grow beyond their pits onto the surrounding (001) terrace, resulting in more facile interdot transport and the "protective" effect of the pits is lost.

Up to now we have presented the results for the growth of QDs on pit-in-terrace patterns, Figure 6-12 presents AFM micrographs showing the results for the growth of $1.3 \mathrm{~nm}$ of $\mathrm{Si}_{0.5} \mathrm{Ge}_{0.5}$ with varying $\mathrm{t}_{\text {anneal }}$ at $650{ }^{\circ} \mathrm{C}$ on quasi-sinusoidal growth surfaces. For patterns with $\lambda p=150 \mathrm{~nm}, 175 \mathrm{~nm}$ and 200nm, QDs are observed to bifurcate and shift to form in every saddle point instead of the pit-bottoms, with the highest uniformity in size and site occupancy observed for $\lambda \mathrm{p}=150 \mathrm{~nm}$ at tanneal $=5 \mathrm{~min}$. More on this observation of the shift in site-selection is presented in Section 6.9.

For $\lambda_{\mathrm{p}}=150 \mathrm{~nm}$, at $\mathrm{t}_{\mathrm{anneal}}=2 \mathrm{~min} 30 \mathrm{sec}$, the QDs form randomly within the pit sidewalls. However, with continual consumption of WL with the increase in $\mathrm{t}_{\text {anneal }}$, the QDs coarsen at the saddle points of the pattern. At longer $t_{\text {anneal }}(10 \mathrm{~min})$ the uniformity is observed to 
(a)

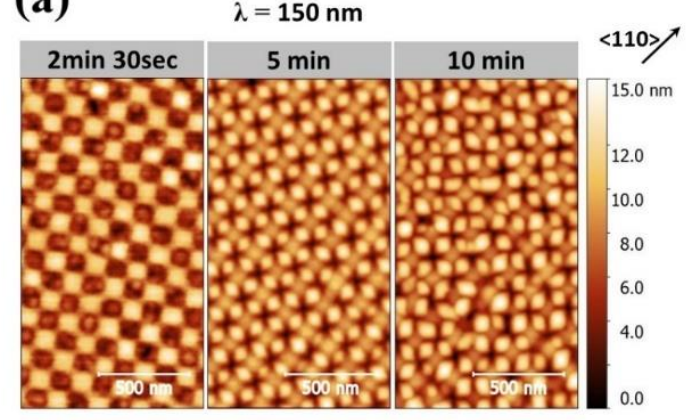

(b)

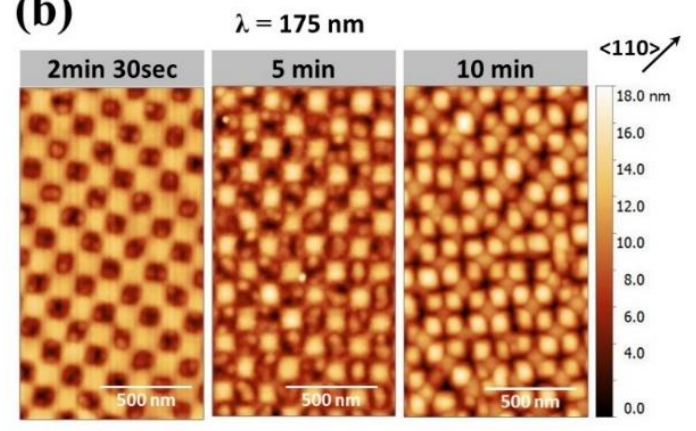

(c)

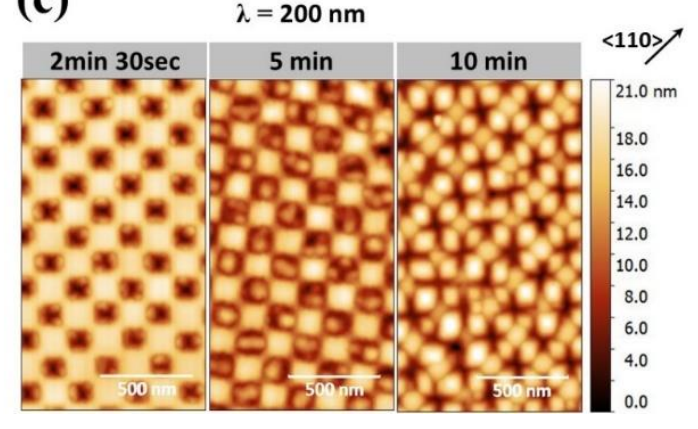

Figure 6-12: Comparison of AFM topography scans of quasi-sinusoidal pattern region at various $\mathrm{t}_{\text {anneal }}$ for $1.3 \mathrm{~nm} \mathrm{Si} 0.5 \mathrm{Ge}_{0.5}$ deposited on patterns with pitch (a) $150 \mathrm{~nm}$, (b) $175 \mathrm{~nm}$, and (c) $200 \mathrm{~nm}$.

decay somewhat at $\lambda_{p}=150 \mathrm{~nm}$, possibly due to the ripening effect as $A_{\text {eff }}$ significantly overlaps at this lengthscale for QDs at saddle points. Similar behavior of the QDs coarsening at the saddle points is observed at both $175 \mathrm{~nm}$ and $200 \mathrm{~nm}$ pitch patterns but at comparatively longer $t_{\text {anneal }}$ suggesting surface kinetics plays a key role. The results here 
clearly suggest that the observation of QDs at saddle points, on quasi-sinusoidal pattern regions, is very sensitive to the anneal time and temperature which in fact governs the overall growth kinetics. For the observation of highly uniform QDs coarsening at saddle points, balance of anneal time, anneal temperature and pattern pitch is critical. For instance, in our work we observed highly uniform array of QDs coarsening at saddle points for $\lambda_{\mathrm{p}}=$ $150 \mathrm{~nm}$ with $\mathrm{t}_{\text {anneal }}=5 \mathrm{~min}$ at $650{ }^{\circ} \mathrm{C}$.

\subsection{Effects of varying Sio.sGe0.5 alloy thickness}

SiGe alloy layers epitaxially grown on a $\mathrm{Si}(001)$ substrate form faceted $3 \mathrm{D}$ islands in order to relax the stored elastic energy. In the previous section, we discussed the growth of $\mathrm{Si}_{0.5} \mathrm{Ge}_{0.5}$ alloy layer with various $\mathrm{t}_{\text {anneal }}$ at $650^{\circ} \mathrm{C}$ and a constant alloy thickness of $1.3 \mathrm{~nm}$. Crucially, this is below the equilibrium wetting layer (WL) thickness, $\mathrm{h}_{\mathrm{wl}}=1.6 \mathrm{~nm}$, for QD formation on planar, monolithic $\mathrm{Si}(001)$. Now in this section, we will vary the alloy thickness, keeping $\mathrm{t}_{\text {anneal }}(5 \mathrm{~min})$ and temperature $\left(650^{\circ} \mathrm{C}\right)$ fixed.

For $\mathrm{t}_{\text {anneal }}=5 \mathrm{~min}$ at $650^{\circ} \mathrm{C}$ at $1.3 \mathrm{~nm}$ alloy thickness, islands do not form off-pattern, as shown in Figure 6-13(a). As the thickness increases, QD areal density increases, first with the formation of "pre-pyramidal" mounds, which transition to $\{105\}$-faceted pyramidal QDs, followed by a transition to domes, resulting in a bimodal size distribution; Figure 613. For patterned regions, however, due to the modification of chemical potential of the growth surface as discussed in Chapter 4, the WL preferentially thickens in the pit region triggering the formation of QDs well before the critical WL thickness is reached on a flat non-patterned surface. 
(a)
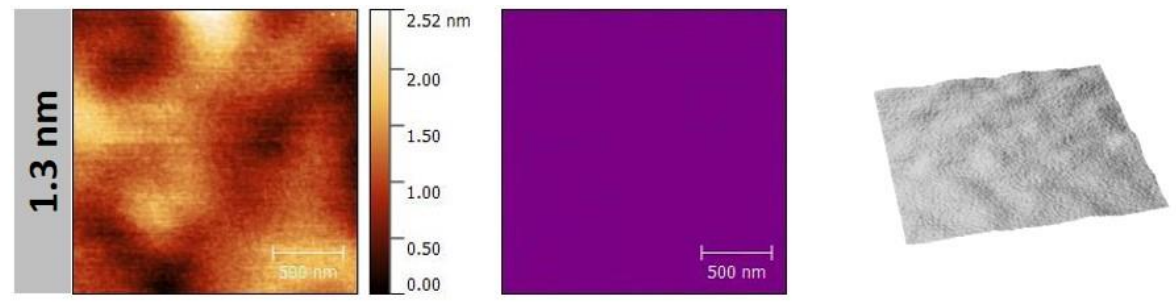

(b)
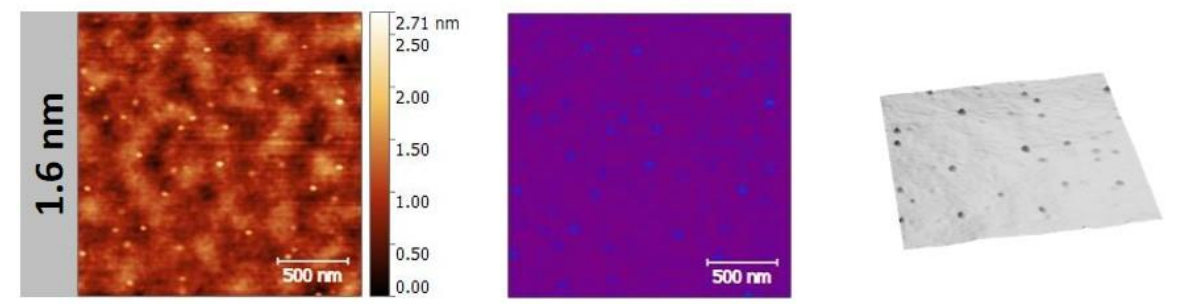

(c)
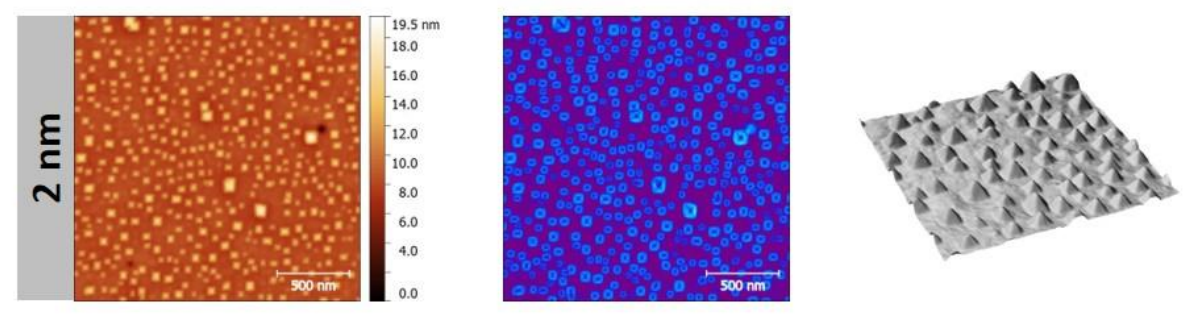

(d)
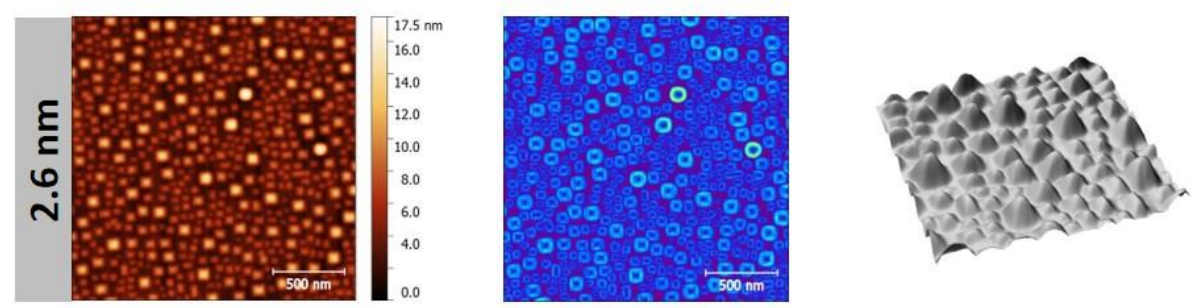

(e)
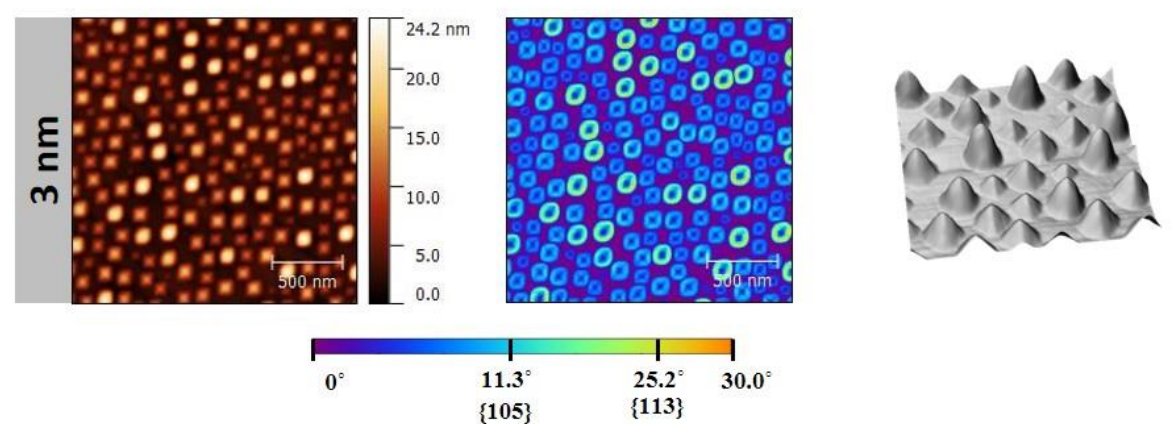

Figure 6-13: $2 \times 2 \mu \mathrm{m}^{2}$ AFM micrographs (left) along with local slope images (middle) and 3D perspective view (right) of $\mathrm{Si}_{0.5} \mathrm{Ge}_{0.5}$ alloy deposited at $450{ }^{\circ} \mathrm{C}$ on a non-patterned $\mathrm{Si}(001)$ substrate, annealed at $650{ }^{\circ} \mathrm{C}$ for tanneal $=5 \mathrm{~min}$ with alloy thickness of (a) $1.3 \mathrm{~nm}$, (b) $1.6 \mathrm{~nm}$, (c) $2 \mathrm{~nm}$, (d) $2.6 \mathrm{~nm}$, and (e) $3 \mathrm{~nm}$. 
Results for the growth of $1.3 \mathrm{~nm}$ vs $2.6 \mathrm{~nm}$ thick $\mathrm{Si}_{0.5} \mathrm{Ge}_{0.5}$ alloy on constant dose pattern fields are presented in Figure 6-14. For $\lambda_{\mathrm{p}}=100 \mathrm{~nm}, 150 \mathrm{~nm}$ and $200 \mathrm{~nm}$ the AFM scans were taken from a constant dose region of $2 \times 10^{-3} \mathrm{pC}, 2.5 \times 10^{-3} \mathrm{pC}$ and $4.5 \times 10^{-3} \mathrm{pC}$

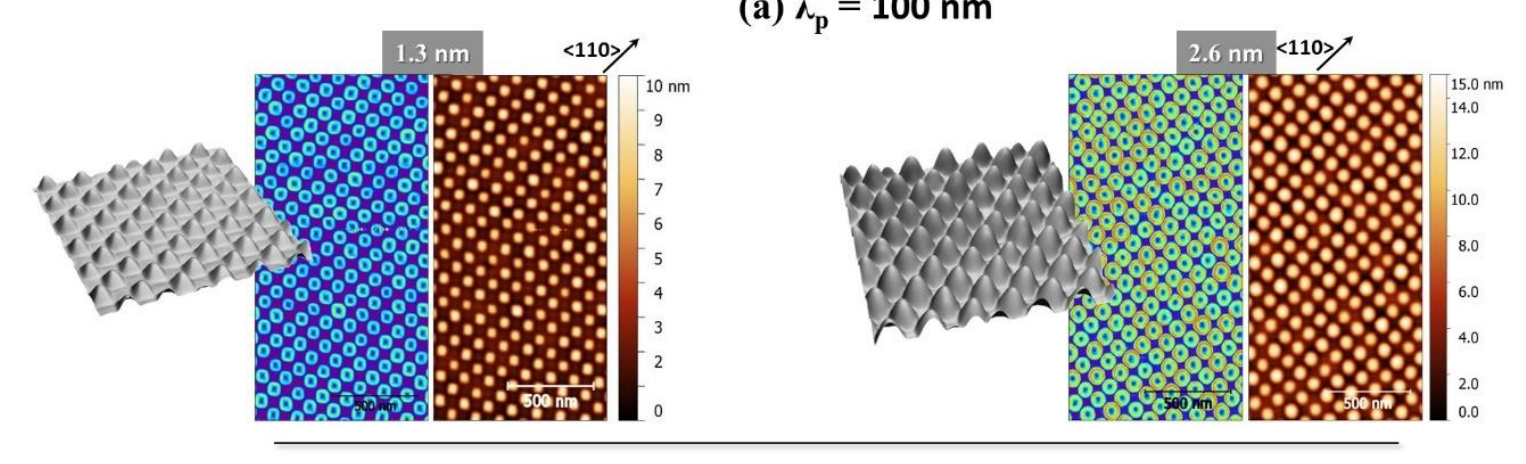

(a) $\lambda_{\mathrm{p}}=100 \mathrm{~nm}$

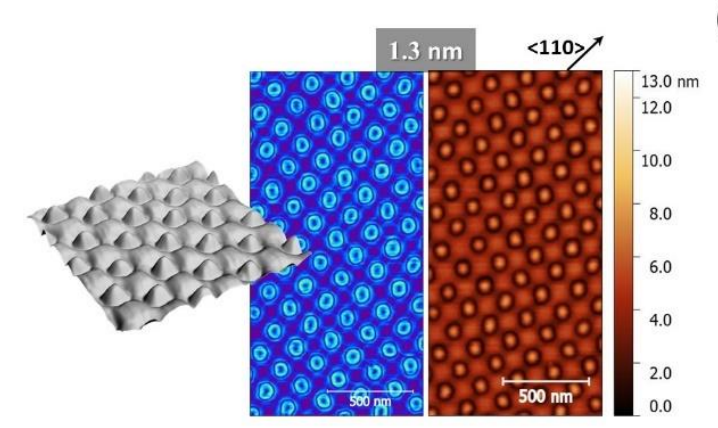

(b) $\lambda_{p}=150 \mathrm{~nm}$

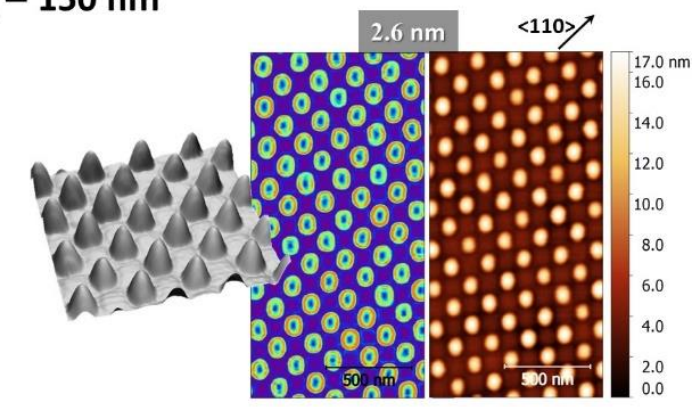

(c) $\lambda_{\mathrm{p}}=200 \mathrm{~nm}$
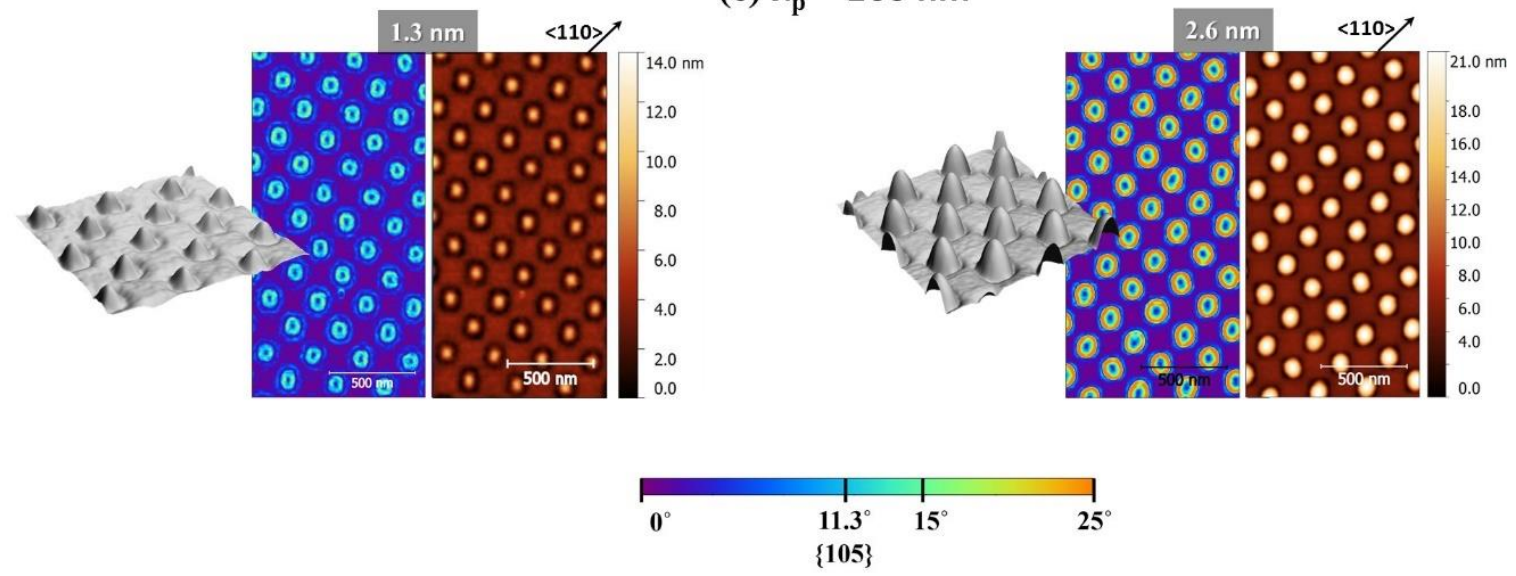

Figure 6-14: Comparison of AFM topography images for $1.3 \mathrm{~nm}$ and $2.6 \mathrm{~nm}$ of $\mathrm{Si}_{0.5} \mathrm{Ge}_{0.5}$ alloy with their corresponding local area slope image and $3 \mathrm{D}$ perspective image for patterns with pitch (a) $100 \mathrm{~nm}$, (b) $150 \mathrm{~nm}$, and (c) $200 \mathrm{~nm}$. $\left(2 \times 10^{-3} \mathrm{pC}, 2.5 \times 10^{-3} \mathrm{pC}\right.$ and $4.5 \times 10^{-3}$ $p C$ for pitch 100, 150 and $200 \mathrm{~nm}$ respectively) 
respectively, such that the initial pit morphology is nominally same in for better comparison. For all the pitch patterns with $1.3 \mathrm{~nm}$ of alloy thickness, see the left side Figure 6-14, QDs are $\{105\}$ faceted pyramids whereas for $2.6 \mathrm{~nm}$ of alloy thickness, QDs are mostly domes with steeper facets, see the right side Figure 6-14. As discussed in Chapter 1 regarding the morphological evolution, for $2.6 \mathrm{~nm}$ thick alloy the pyramidal structure has already transitioned to dome structures in the pits to relieve the comparatively higher strain energy in the thicker alloy. However, for the $2.6 \mathrm{~nm}$ thick alloy off the patterned region the structures are mostly pyramidal. A more rapid transformation to the dome morphology in the patterned regions is consistent with enhanced mass capture by the pits, see Figure 615.
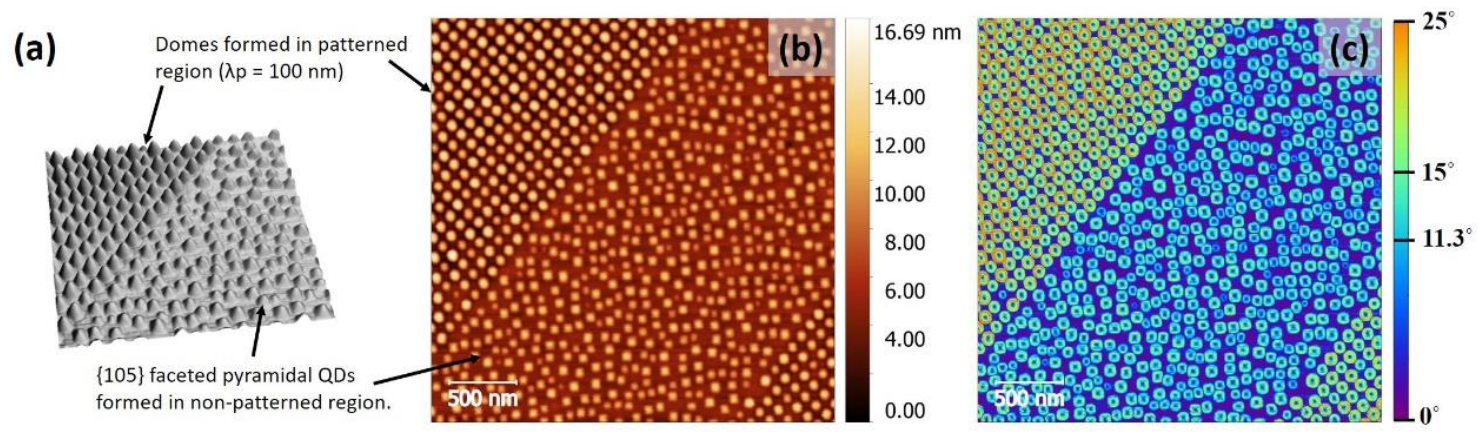

Figure 6-15: (a) 3D perspective image, (b) AFM topography image, and (c) local area slope image comparing patterned and non-patterned region for $2.6 \mathrm{~nm}$ thick $\mathrm{Si}_{0.5} \mathrm{Ge}_{0.5}$ alloy annealed at $650{ }^{\circ} \mathrm{C}$ for $5 \mathrm{~min}$.

Volumetric analysis of QDs shown in Figure 6-14 is compiled in Figure 6-16. Similar to the analysis method discussed earlier, we masked all the QDs within a $2 \times 2 \mu \mathrm{m}^{2}$ AFM image such that 330,150 and 80 QDs were selected for patterns with pitch of 100, 150 and 200 $\mathrm{nm}$ respectively. The results for the QD volume distribution in absolute units is given in left side of the Figure 6-16, while the normalized distribution plots are shown on the right side. The statistical data of the volume distribution curves is given in Table 6-2. 
Table 6-2: Statistical data for the distribution width, both absolute and normalized units, of probability density plots shown in Figure 6-16.

\begin{tabular}{|c|c|c|c|c|c|c|}
\hline $\begin{array}{c}\text { Pitch } \\
(\boldsymbol{\lambda})\end{array}$ & $\begin{array}{c}\text { Thickness } \\
(\mathbf{n m})\end{array}$ & $\begin{array}{c}\text { Mean } \\
\text { Volume }<\mathbf{V}> \\
\mathbf{\mathbf { x 1 0 } ^ { - 2 3 }} \mathbf{~ m}^{\mathbf{3}}\end{array}$ & $\begin{array}{c}\text { Std. } \\
\text { Deviation } \\
\mathbf{( S D} \text { or } \boldsymbol{\sigma})\end{array}$ & $\begin{array}{c}\text { FWHM } \\
\mathbf{( 2 . 3 5 5} \boldsymbol{\sigma})\end{array}$ & $\begin{array}{c}\text { Relative } \\
\mathbf{S D}(\boldsymbol{\sigma} \mathbf{R})\end{array}$ & $\begin{array}{c}\text { Normalized } \\
\text { FWHM } \\
\mathbf{( 2 . 3 5 5 \sigma \mathbf { r } )}\end{array}$ \\
\hline \multirow{2}{*}{100} & 1.3 & 1.199 & 0.190 & 0.446 & 0.158 & 0.372 \\
\cline { 2 - 7 } & 2.6 & 3.042 & 0.954 & 2.247 & 0.314 & 0.739 \\
\hline \multirow{2}{*}{150} & 1.3 & 1.636 & 0.228 & 0.538 & 0.140 & 0.329 \\
\cline { 2 - 7 } & 2.6 & 4.873 & 1.452 & 3.419 & 0.298 & 0.702 \\
\hline \multirow{2}{*}{200} & 1.3 & 2.879 & 0.321 & 0.756 & 0.112 & 0.263 \\
\cline { 2 - 7 } & 2.6 & 11.497 & 0.992 & 2.337 & 0.086 & 0.203 \\
\hline
\end{tabular}

In Figure 6-16, the volume distribution plots on the left side clearly show for all the patterns that the mean QD volume for the thicker alloy sample is higher, consistent with more deposited material. Comparing the normalized probability density, for $\lambda_{\mathrm{p}}=100 \mathrm{~nm}$, the distribution width of $2.6 \mathrm{~nm}$ sample is broader compared to $1.3 \mathrm{~nm}$, see Figure 6-16(a). The broadening is due to ripening which is more effective for $2.6 \mathrm{~nm}$ sample because the rate of material incorporation in dome structures are faster compared to pyramidal structures and further, the domes have actually grown beyond their pits onto the surrounding (001) terrace. Similar effect is observed at $\lambda_{\mathrm{p}}=150 \mathrm{~nm}$ as well where pattern with dome structures (2.6 $\mathrm{nm}$ sample) are found to have broader distribution due to enhanced ripening effect. However, at $\lambda_{\mathrm{p}}=200 \mathrm{~nm}$, the distribution width for $2.6 \mathrm{~nm}$ sample is nominally similar to that of the $1.3 \mathrm{~nm}$ sample, see Figure 6-16(c). As discussed regarding the growth kinetics in the previous section and Figure 6-11, at $\lambda_{\mathrm{p}}=200 \mathrm{~nm}$, ripening and growth of the QDs is basically limited by surface kinetics. 
(a)

$330 \mathrm{QD}$ population for pattern pitch $=100 \mathrm{~nm}$; dose $=2 \times 10^{-3} \mathrm{pC}$

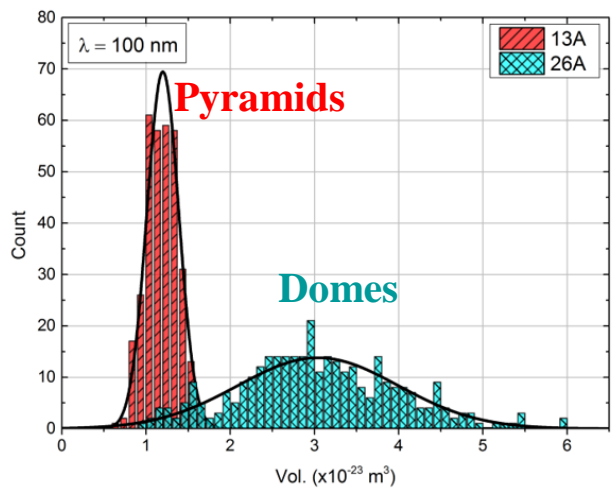

$330 \mathrm{QD}$ population for pattern pitch $=100 \mathrm{~nm} ;$ dose $=2 \times 10^{-3} \mathrm{pC}$

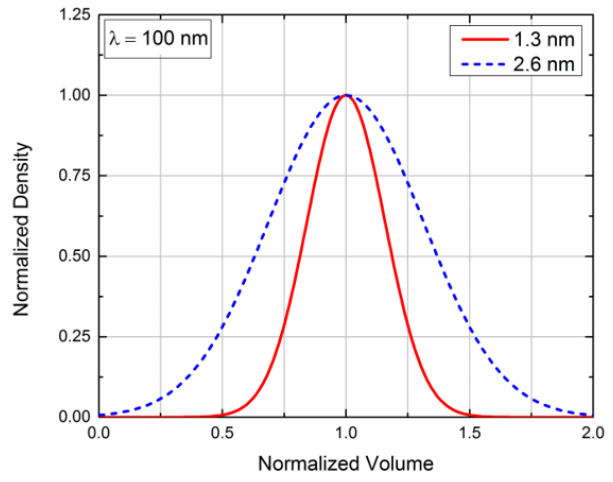

(b)
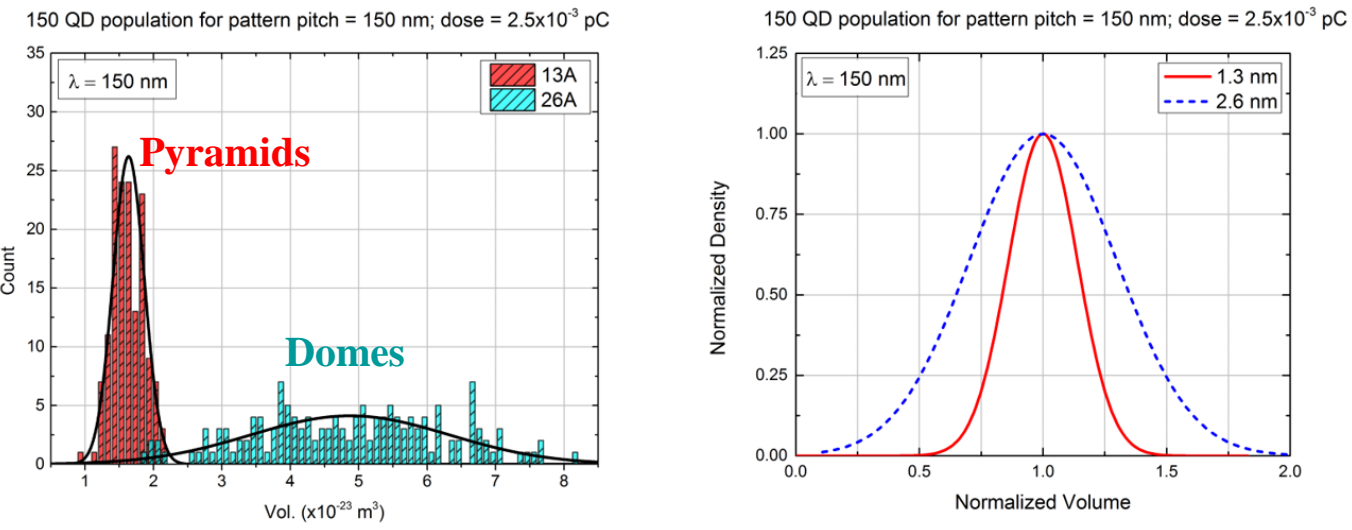

(c)
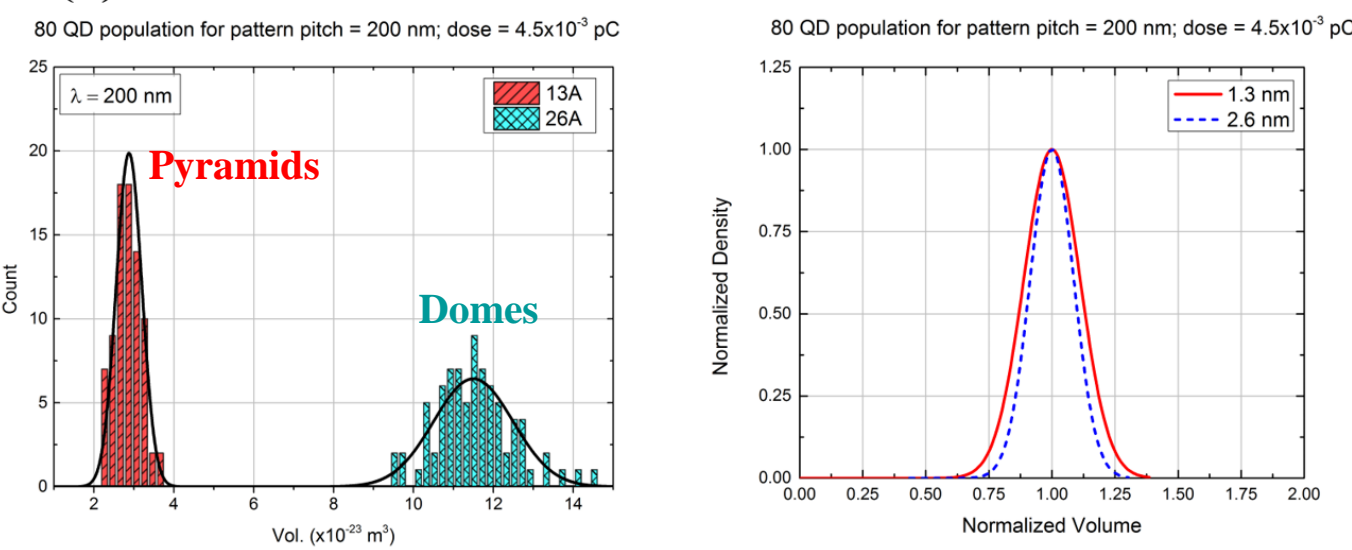

Figure 6-16: Comparison of volume distribution of QDs for the deposition of $1.3 \mathrm{~nm}$ vs $1.6 \mathrm{~nm}$ of $\mathrm{Si}_{0.5} \mathrm{Ge}_{0.5}$ in absolute and normalized scale for pattern with pitch (a) $100 \mathrm{~nm}$, (b) $150 \mathrm{~nm}$, and (c) $200 \mathrm{~nm}$. 
(a) $\lambda=150 \mathrm{~nm}$

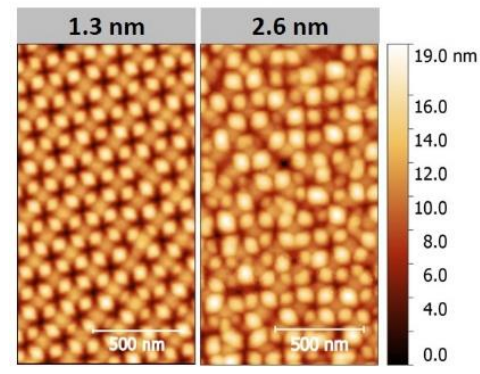

(b) $\quad \lambda=175 \mathrm{~nm}$

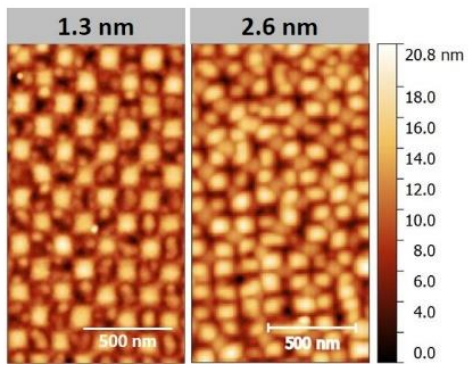

(c) $\lambda=200 \mathrm{~nm}$

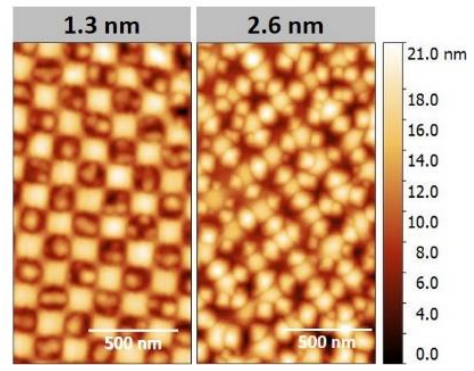

Figure 6-17: AFM topography scans of quasi-sinusoidal pattern region at $650{ }^{\circ} \mathrm{C}$ with $\mathrm{t}_{\text {anneal }}$ $=5 \mathrm{~min}$, comparing $1.3 \mathrm{~nm}$ vs $2.6 \mathrm{~nm}$ thick $\mathrm{Si}_{0.5} \mathrm{Ge}_{0.5}$ alloy deposited on patterns with pitch (a) $150 \mathrm{~nm}$, (b) $175 \mathrm{~nm}$, and (c) $200 \mathrm{~nm}$.

Figure 6-17 presents AFM micrographs comparing the results for the growth of $1.3 \mathrm{~nm}$ vs $2.6 \mathrm{~nm}$ thick $\mathrm{Si}_{0.5} \mathrm{Ge}_{0.5}$ alloy at $650{ }^{\circ} \mathrm{C}$ with $\mathrm{t}_{\text {anneal }}=5 \mathrm{~min}$, on the quasi-sinusoidal growth surface. For $\lambda_{p}=150 \mathrm{~nm}$, increasing the material thickness degrades the uniformity of QDs forming at saddle points. For $\lambda_{\mathrm{p}}=175 \mathrm{~nm}$ and $200 \mathrm{~nm}$, QDs for the $1.3 \mathrm{~nm}$ sample initially form randomly at the pit sidewalls, whereas for the thicker $2.6 \mathrm{~nm}$ alloy site-selection shifts from pit-sidewall to the saddle point. This is similar to the results obtained at higher $\mathrm{t}_{\text {anneal }}$ $=10 \mathrm{~min}$ for $1.3 \mathrm{~nm}$ thick alloy samples; compare Figure 6-12 and Figure 6-17, suggesting strain relaxation plays a critical role in QD site selection. Interestingly, Figure 6-17 also shows that when QDs form at the saddle points they retain their $\{105\}$-faceted morphology, while the QDs in the pit-in-terrace regions of the same specimen transition to dome clusters. This is due to the bifurcation in volume associated with the saddle-point location, keeping the QDs below the critical volume for the shape transition. 


\subsection{Pattern Orientation: $\langle 110>$ vs $<100>$}

All the patterned regions discussed up to now have been aligned along the <110> crystallographic direction. Here, we present the growth results for $1.3 \mathrm{~nm}$ thick $\mathrm{Si}_{0.5} \mathrm{Ge}_{0.5}$ alloys annealed at $650{ }^{\circ} \mathrm{C}$ for $5 \mathrm{~min}$ on patterned regions aligned along the <100> crystallographic direction (angle between $\langle 110\rangle$ and $\langle 100\rangle$ is $45^{\circ}$ ). Figure 6-18 compares both the AFM micrographs and volume distribution of QDs grown on patterns (constant dose pattern region) along <110> vs. patterns along $\langle 100\rangle$. The statistical data of the volume distribution curves in both absolute and normalized scale is given in Table 6-3.

Table 6-3: Statistical data for the distribution width, both absolute and normalized units, of probability density plots are given (note the absolute PDF plots are not given in Figure 618).

\begin{tabular}{|c|c|c|c|c|c|c|}
\hline $\begin{array}{l}\text { Pitch } \\
(\lambda)\end{array}$ & Orientation & $\begin{array}{c}\text { Mean } \\
\text { Volume } \\
<\mathrm{V}> \\
\times 10^{-23} \mathbf{m}^{3}\end{array}$ & $\begin{array}{c}\text { Std. } \\
\text { Deviation } \\
(\text { SD or } \sigma)\end{array}$ & $\begin{array}{l}\text { FWHM } \\
(2.355 \sigma)\end{array}$ & $\begin{array}{c}\text { Relative } \\
\text { SD (RSD } \\
\left.\text { or } \sigma_{R}\right)\end{array}$ & $\begin{array}{c}\text { Normalized } \\
\text { FWHM } \\
\left(2.355 \sigma_{\mathrm{R}}\right)\end{array}$ \\
\hline \multirow{2}{*}{100} & $\langle 110\rangle$ & 1.199 & 0.190 & 0.446 & 0.158 & 0.372 \\
\hline & $\langle 100\rangle$ & 0.959 & 0.152 & 0.357 & 0.158 & 0.372 \\
\hline \multirow{2}{*}{150} & $\langle 110\rangle$ & 1.636 & 0.228 & 0.538 & 0.140 & 0.329 \\
\hline & $\langle 100\rangle$ & 1.762 & 0.250 & 0.590 & 0.142 & 0.335 \\
\hline \multirow{2}{*}{200} & $\langle 110\rangle$ & 2.879 & 0.321 & 0.756 & 0.112 & 0.263 \\
\hline & $\langle 100\rangle$ & 2.986 & 0.302 & 0.712 & 0.101 & 0.238 \\
\hline
\end{tabular}

In all the pitches examined, the mean volumes of the QDs were nominally similar for both the orientations of the pattern. The normalized distribution width $\left(\mathrm{FWHM}_{\mathrm{norm}}\right)$ is nominally similar for patterns with different orientation but the same pitch, which clearly suggests 
that there is no significant variation in the QD evolution for patterns with different orientation i.e., along $<110>$ vs $<100>$.
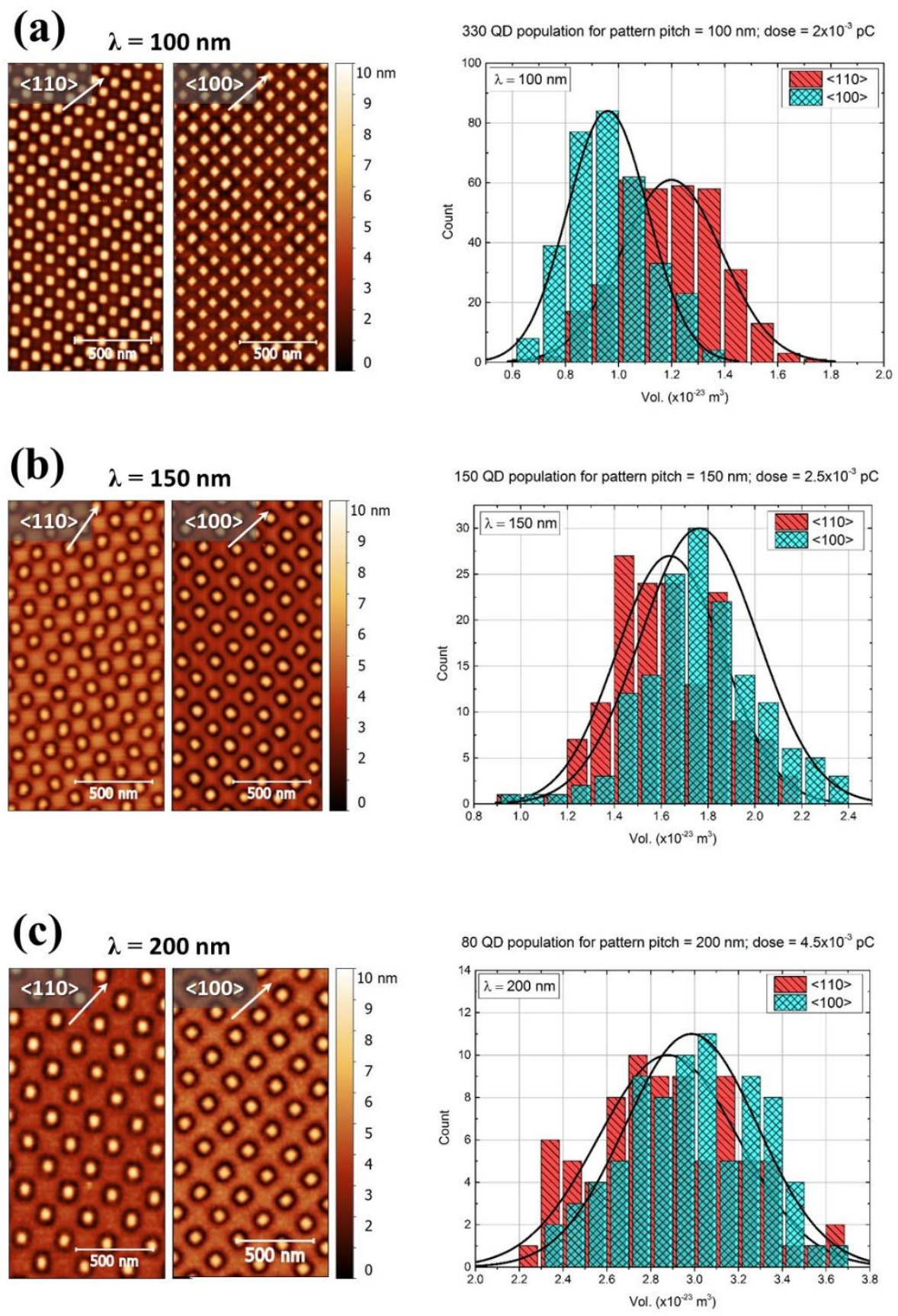

Figure 6-18: Comparison of AFM image (left) along with volume distribution (right) of QDs for the deposition of $1.3 \mathrm{~nm}$ thick $\mathrm{Si}_{0.5} \mathrm{Ge}_{0.5}$ alloy annealed at $650{ }^{\circ} \mathrm{C}$ with $\mathrm{t}_{\text {anneal }}=$ $5 \mathrm{~min}$ for patterns with pitch (a) $100 \mathrm{~nm}$, (b) $150 \mathrm{~nm}$, and (c) $200 \mathrm{~nm}$. 
However, when comparing the quasi-sinusoidal growth surface with orientation along $<110>$ vs $<100>$ the results were interesting: QDs grew randomly for patterns along <100> whereas for patterns along $\langle 110\rangle$, QDs formed at saddle points with high uniformity, see Figure 6-19. Throughout the growth conditions explored here unlike patterns along $<110\rangle$, we never observed localization of QDs at saddle points for patterns oriented along <100>. This could suggest that saddle point nucleation is sensitive to surface anisotropy, e.g., the saddle points in $\langle 110\rangle$ patterns are conducive to the formation of $\{105\}$ facets in the quantum dots. However, we also note that the underlying pattern itself breaks down in the TR of the $<100>$-oriented patterns.

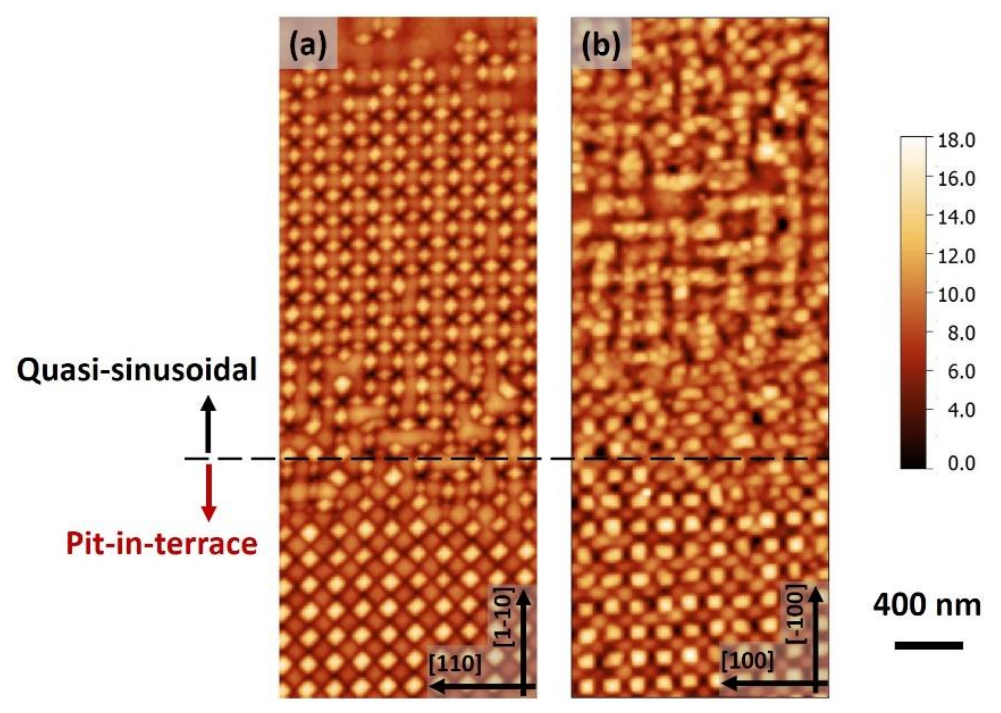

Figure 6-19: AFM micrographs comparing the effect of pattern orientation for the pit-interrace and quasi-sinusoidal patterned region with $\lambda_{\mathrm{p}}=150 \mathrm{~nm}$, (a) pattern oriented along $\langle 110\rangle$, and (b) pattern oriented along <100>. 


\subsection{Estimation of surface diffusion length}

So far we have discussed the QD growth regarding the effects of pattern morphology, alloy thickness, pattern orientation, annealing time and temperature. This section presents our results and discussion regarding the estimation of surface diffusion length for the adatom species during annealing which governs the overall growth kinetics.

Figure 6-20 shows AFM topography scans along with local-area slope images and 3D perspective views for $2.6 \mathrm{~nm}$ thick $\mathrm{Si}_{0.5} \mathrm{Ge}_{0.5}$ alloys annealed at $650{ }^{\circ} \mathrm{C}$ for $5 \mathrm{~min}$ on patterns with $\lambda_{\mathrm{p}}=200 \mathrm{~nm}$. Figure 6-20(a) shows the results for a low-dose patterned region $\left(3.75 \times 10^{-3} \mathrm{pC}\right)$ along $\langle 110\rangle$, where the initial size of the pit-opening $(\mathrm{W})$ was $98.5 \pm 2.3$ $\mathrm{nm}$. Figure 6-20(b) shows results for a higher-dose patterned region $\left(6.75 \times 10^{-3} \mathrm{pC}\right)$ along $\langle 110\rangle$, where $\mathrm{W}=114.9 \pm 2.3 \mathrm{~nm}$. And finally, in Figure 6-20(c) the results presented are for same dose region $\left(3.75 \times 10^{-3} \mathrm{pC}\right)$ as shown in Figure 6-20(a) but here the pattern is along $<100>$ and $\mathrm{W}=100.76 \pm 2.45 \mathrm{~nm}$. Details on the relation between $\mathrm{W}$ and dose were presented in Chapter 5. All the scans shown in Figure 6-20 were taken from a single sample, such that all three regions with different dose underwent the identical process cycle.

For the alloy thickness of $2.6 \mathrm{~nm}$, we have already showed that QDs are observed to form off the patterned regions, see Figure 6-13. The formation of secondary QDs on flat crown regions as shown in Figure 6-20(a) clearly indicates that the equilibrium WL thickness was surpassed in these regions. The fully-ordered coexistence of pyramids on crowns and domes inside pits is interesting and represents the only instance in this research that crown nucleation has been observed. Coexistence implies that the transfer of adatoms to the pits from the crown regions is restricted by the surface kinetics, so that the wetting layer on the crown is allowed to thicken. For instance, patterns with comparatively larger pit-opening 
did not yield any secondary QD nucleation on crown regions, see Figure 6-20(b). Note that as $\mathrm{W}$ increases, the effective edge to edge distance $\left(\mathrm{d}_{\mathrm{e}-\mathrm{e}}\right)$ between the two pits across the crown region decreases. The reduction of $\mathrm{d}_{\mathrm{e}-\mathrm{e}}$ means that the adatoms need to travel shorter

(a) $\lambda_{\mathrm{p}}=200 \mathrm{~nm} ; W=98.55 \pm 2.31 \mathrm{~nm}$

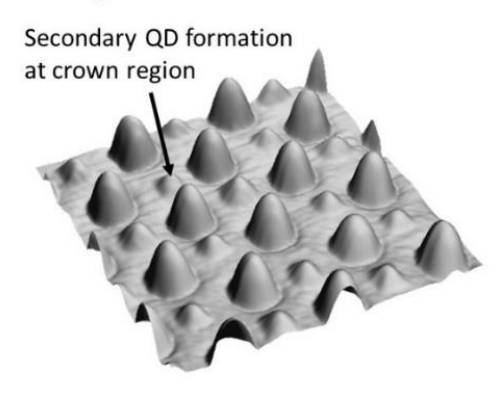

(b) $\lambda_{p}=200 \mathrm{~nm} ; W=114.93 \pm 2.26 \mathrm{~nm}$

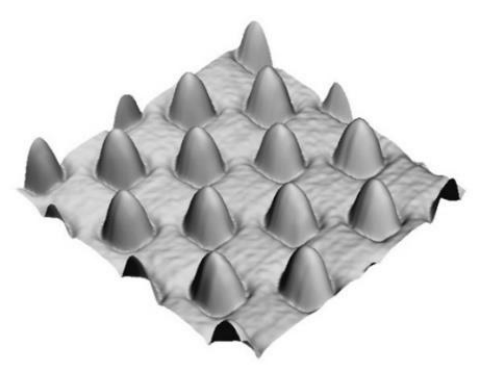

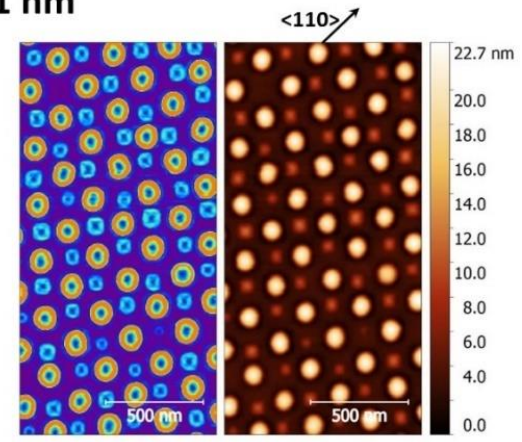
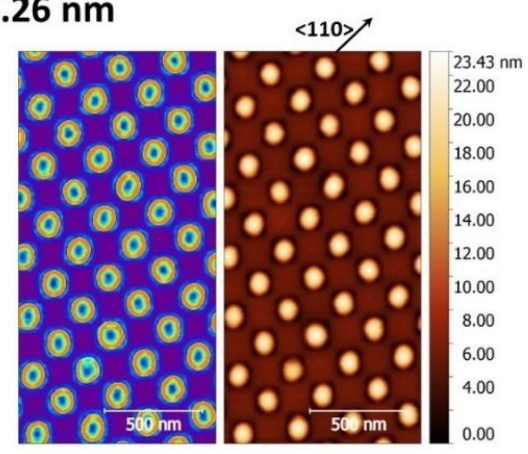

(c) $\lambda_{p}=200 \mathrm{~nm} ; W=100.76 \pm 2.45 \mathrm{~nm}$
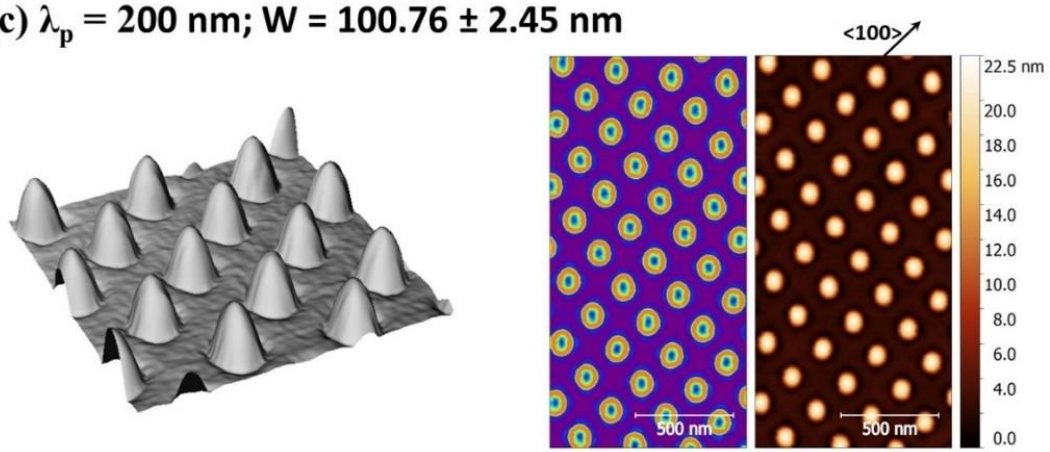

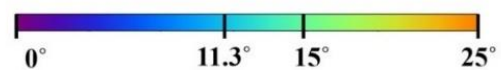

Figure 6-20: AFM 3D perspective image along with topography and local area slope image showing the comparative results for $2.6 \mathrm{~nm}$ thick $\mathrm{Si}_{0.5} \mathrm{Ge}_{0.5}$ alloy growth on $\lambda_{\mathrm{p}}=200 \mathrm{~nm}$ region with varying pit-opening size $-\mathrm{W}\left(t_{\text {anneal }}=5 \min\right.$ at $\left.650^{\circ} \mathrm{C}\right)$. (a) and (b) are for patterns oriented along $\langle 110\rangle$, (c) is pattern oriented along $\langle 100\rangle$. 
distance from the crown regions to get captured by the pits, therefore reducing the probability of secondary nucleation on the crown; see Figure 6-21 for more details

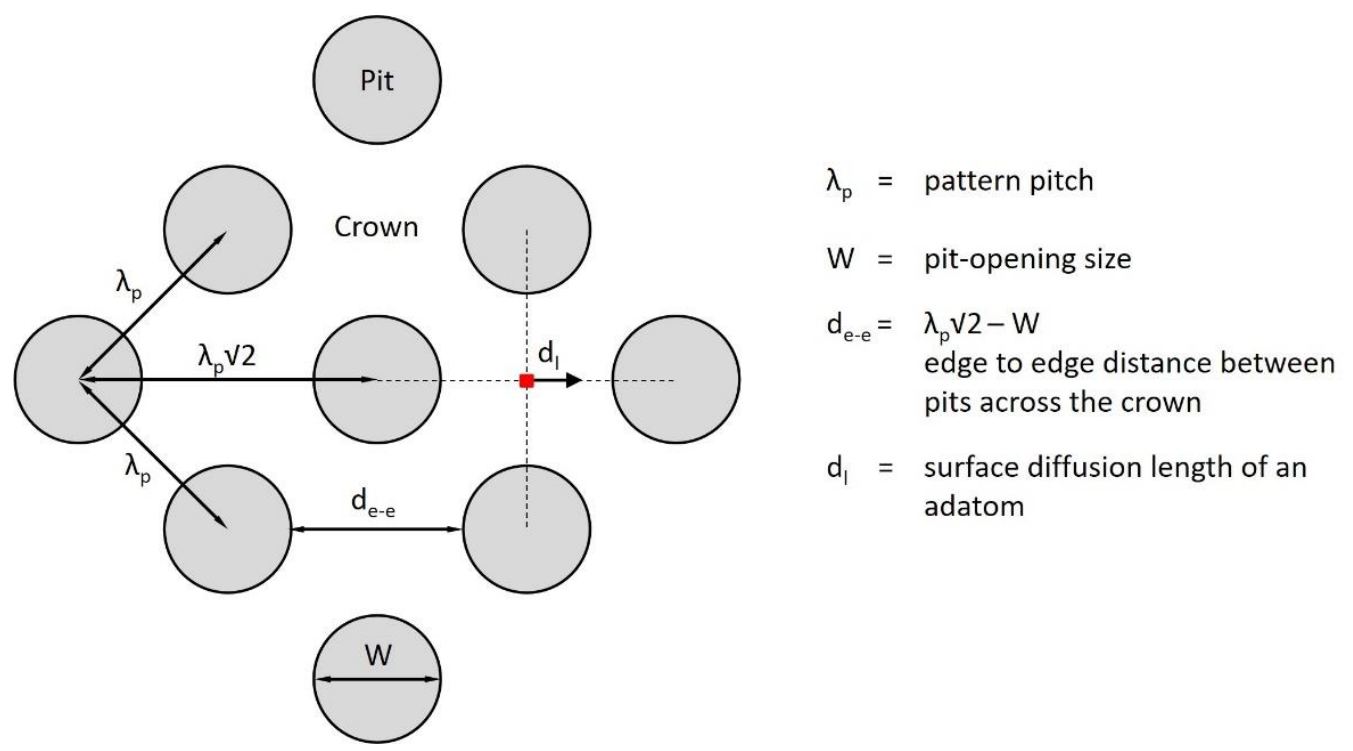

Figure 6-21: Schematics of patterned regions showing the diffusion length parameters along with pattern pitch $\left(\lambda_{\mathrm{p}}\right)$ and pit-opening $(\mathrm{W})$.

Figure 6-21 presents the schematics of the patterned regions showing the diffusion length parameters from the crown region calculated by simple geometry. We that the surface diffusion length of adatoms $\left(\mathrm{d}_{1}\right)$ needs to be greater than $\mathrm{d}_{\mathrm{e}-\mathrm{e}} / 2$ to avoid any secondary formation of QDs on crown region. Provided that all other experimental variables remain same, then the condition at which there will be no existence of secondary QDs on crown region will be given by,

$$
\mathrm{d}_{\mathrm{l}} \geq \frac{\mathrm{d}_{\mathrm{e}-\mathrm{e}}}{2}=\frac{\lambda_{\mathrm{p}} \sqrt{2}-\mathrm{W}}{2}
$$

And the condition at which secondary QDs on crown region will exist is given by, 


$$
\mathrm{d}_{\mathrm{l}}<\frac{\mathrm{d}_{\mathrm{e}-\mathrm{e}}}{2}=\frac{\lambda_{\mathrm{p}} \sqrt{2}-\mathrm{W}}{2}
$$

Both Equation 6-4 and Equation 6-5 outline the requirement of surface diffusion length for the coexistence of secondary QDs on the crown regions. With the results shown in Figure 6-20(a) and (b), we do not observe any secondary QDs for $\mathrm{W}>98.5 \pm 2.3 \mathrm{~nm}$. Therefore, with reference to Equation 6-4 and Equation 6-5, with $\lambda_{\mathrm{p}}=200 \mathrm{~nm}$ the calculated surface diffusion length is $\mathrm{d}_{1} \approx 92 \mathrm{~nm}$.

However, when the pattern orientation is changed from $\langle 110\rangle$ to $\langle 100\rangle$, even with the similar pit-opening size (same dose) there is no observation of secondary QDs for pattern along $\langle 100\rangle$, see Figure 6-20(c). We attribute this effect to the diffusional anisotropy of Si and Ge adatoms on the surface. The diffusion of adatoms on Si or Ge surface is strongly anisotropic due to the $2 \mathrm{x} 1$ reconstruction, such that the adatoms diffuse preferentially along $<110>$ in-plane directions. With reference to the energy barrier values defined by Barbato and Cavallotti ${ }^{130}$, for $\mathrm{Si}$ ad-atoms alone an energy barrier value of $0.60 \mathrm{eV}$ was calculated for diffusion along $\langle 110\rangle$, while a value of $1.0 \mathrm{eV}$ was calculated for diffusion along $<100>$. Iguain et al. ${ }^{131}$ and Rockett et al. ${ }^{132}$ have also applied similar barrier values in their simulation work that produced results comparable to experimental data of epitaxial growth surfaces. Based on this reported anisotropy, we suggest that when the $d_{\mathrm{e}-\mathrm{e}}$ direction is aligned along <110> (i.e., pattern aligned along <100>) it is easier for adatoms to diffuse from the crown region, limiting the formation of secondary QDs.

Note that the estimated diffusion length of adatoms in our work $(\sim 90 \mathrm{~nm})$ is small compared to the diffusion length of as deposited adatoms at similar growth temperature 
(few hundreds of $\mathrm{nm}^{133}$ ). We attribute this to our growth process where a nominally conformal film (deposited at lower temperature) is annealed at higher temperature to activate surface diffusion and form the QDs, rather than depositing the material at higher temperature to directly grow QDs during the course of deposition. The observation can be simply described in terms of energetics such that the energy barrier for the diffusing species on surface for as grown condition is smaller than that of an annealed condition, see Figure $6-22$.

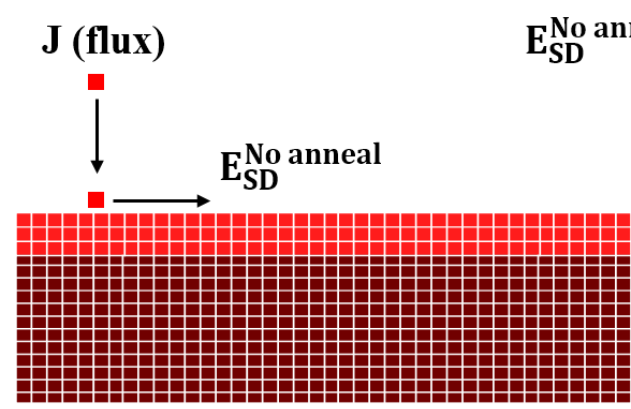

(a)

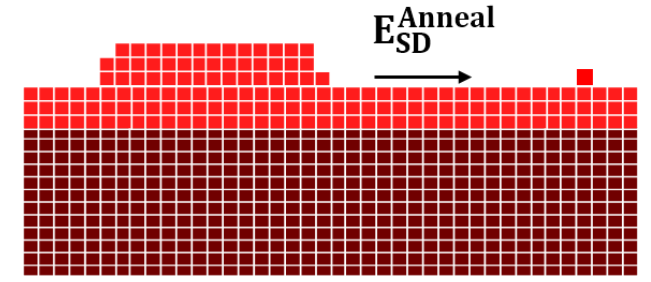

(b)

Figure 6-22: Schematics of the growth conditions showing surface diffusion kinetics for (a) adatom species under a constant deposition flux $\mathrm{J}$, and (b) adatom species during annealing post deposition at lower temperature. $E_{S D}^{\text {No anneal }}$ is the energy barrier for surface adatoms to diffuse and $E_{S D}^{A n n e a l}$ is the energy barrier for the surface adatoms to diffuse post deposition during anneal.

\subsection{Site-selection of $\mathrm{Si}_{0.5} \mathrm{Ge}_{0.5} \mathrm{QDs}$ on patterned region}

Results for the growth of $1.3 \mathrm{~nm}$ thick $\mathrm{Si}_{0.5} \mathrm{Ge}_{0.5}$ alloy with $\mathrm{t}_{\text {anneal }}=5 \mathrm{~min}$ at $650{ }^{\circ} \mathrm{C}$ on the patterned regions as described in Section 6.4 are presented in Figure 6-23, showing AFM topography scans, comparing the TRs for pattern fields with various pitches. Again, W increases along the $[1 \overline{1} 0]$ crystallographic direction. QDs grown on such patterned regions exhibit $\{105\}$-faceted, quasi-pyramidal islands. No QD formation was observed off the 
patterned region, since the film is below the critical wetting layer thickness for island formation on the non-patterned (001) surface, which for our growth conditions was observed to be $\sim 1.6-1.8 \mathrm{~nm}$, see Section 6.6.

For $\lambda_{\mathrm{p}}=100 \mathrm{~nm}$, QDs form at the pit bottoms, up to and including the TR, followed by the disintegration of the pattern and accompanying spatial disorder, see Figure 6-23(a). The results are similar for $\lambda_{p}=125 \mathrm{~nm}$, although QD ordering is preserved further into the TR, see Figure 6-23(b). However, for $\lambda_{p}=150 \mathrm{~nm}$, QD site-selection dramatically changes in transition region, see Figure 6-23(c). At lower $W / \lambda_{\mathrm{p}}$, the QDs are in the pit bottoms. However, when the pattern profile transforms to quasi-sinusoidal, the QDs bifurcate and preferentially form with high regularity on all the saddle points between two nearestneighbor pits. This is not the location of most positive curvature, which occurs at the "crown" - the center of the square connecting four proximal pits, see Figure 6-6(a) inset to clarify the nomenclature. For $\lambda_{p}>150 \mathrm{~nm}$, in the TR, the QDs mostly form in the pit bottoms up to the TR, but in the TR, they form on pit-sidewalls or at random locations, see Figure 6-23(d) and (e).
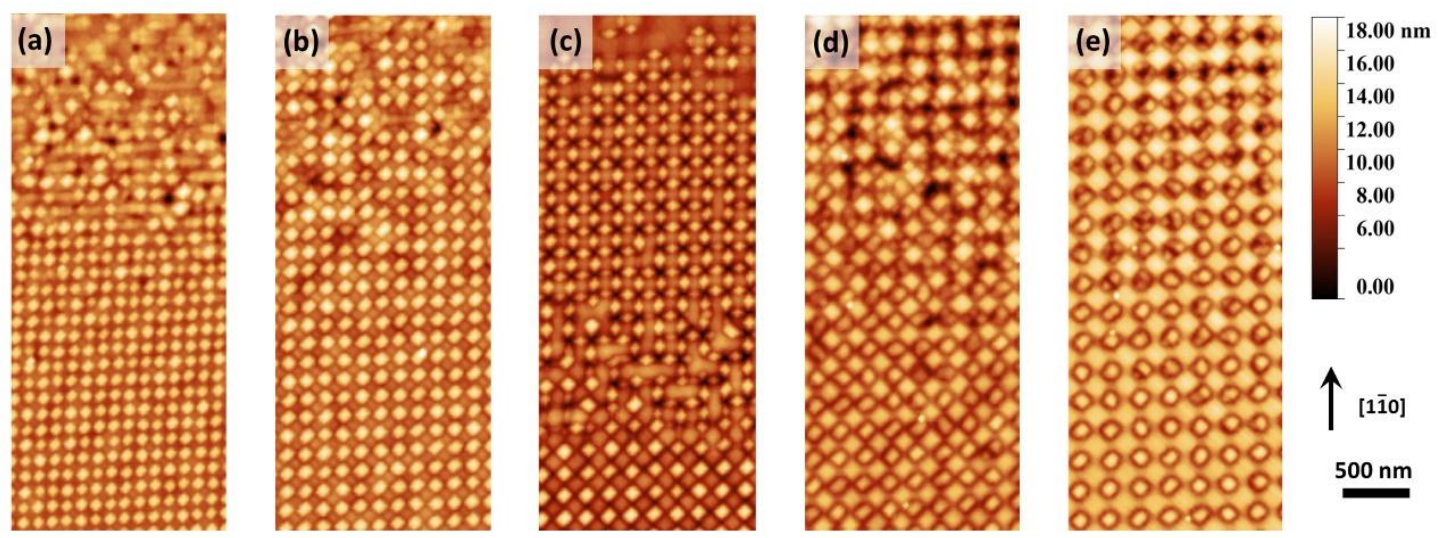

Figure 6-23: AFM micrographs highlighting the transition regions in patterns with $\lambda_{\mathrm{p}}=(\mathrm{a})$ $100 \mathrm{~nm}$, (b) $125 \mathrm{~nm}$, (c) $150 \mathrm{~nm}$, (d) $175 \mathrm{~nm}$, and (e) $200 \mathrm{~nm}$. (1.3 nm thick $\mathrm{Si}_{0.5} \mathrm{Ge}_{0.5}$ alloy layer deposition with $\mathrm{t}_{\text {anneal }}=5 \mathrm{~min}$ at $650{ }^{\circ} \mathrm{C}$ ) 
Figure 6-24 shows AFM micrographs and corresponding local slope maps that compare the QD site selections in the $\lambda_{p}=150 \mathrm{~nm}$ pattern. Figure 6-24(c) and (d) shows the 3D perspective image for the AFM topography scans along with line scans along $<110\rangle$ and $<100>$ in Figure 6-24(e) and (f) respectively. The QDs always exhibit the ubiquitous $\{105\}$ faceting, but the islands forming on saddle regions appear elongated along the two
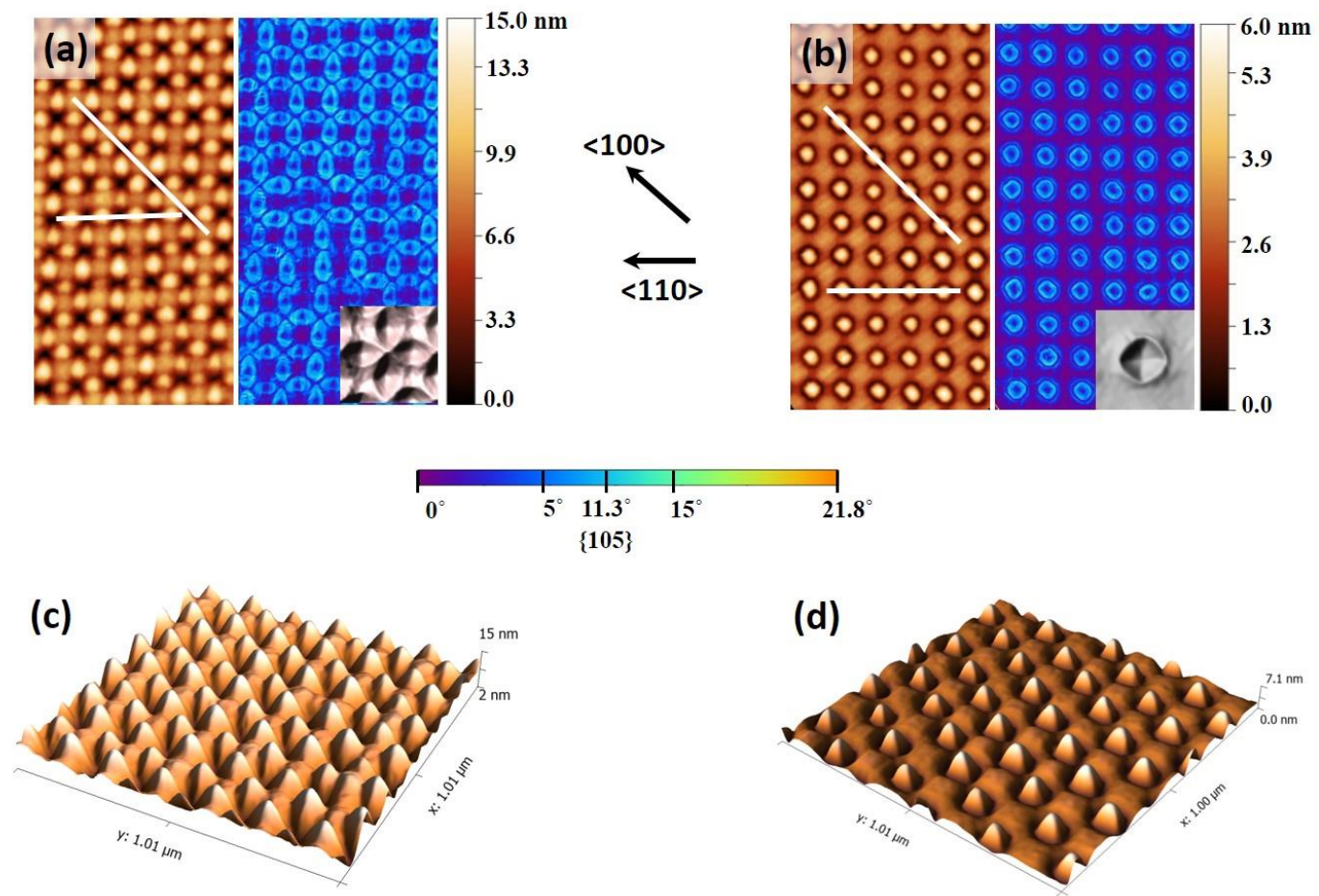

(e)

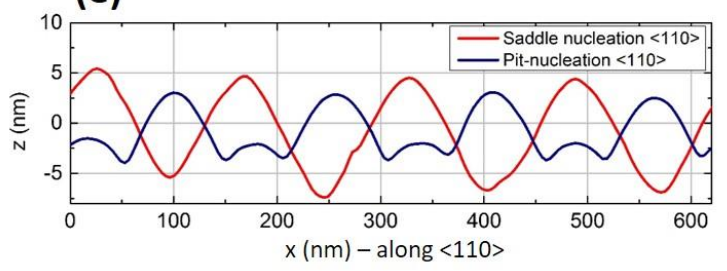

(f)

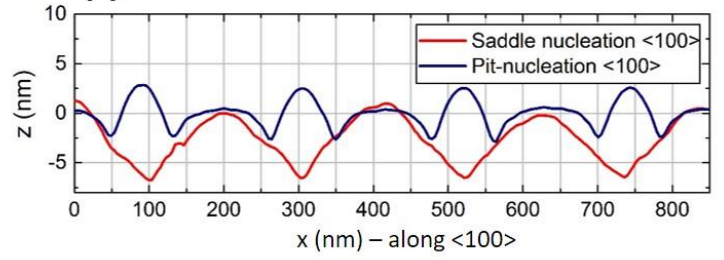

Figure 6-24: AFM micrographs from $\lambda \mathrm{p}=150 \mathrm{~nm}$ pattern, showing (a) saddle nucleation, and (b) pit nucleation. Both topography (left) and local slope (right) images are shown. In (a), the inset shows a close-up of four QDs on the saddle points surrounding a pit. All the QDs are $\{105\}$ faceted. (c) and (d) are 3D perspective image of (a) and (b) AFM topography images respectively. (e) and (f) are Line scan comparisons along $\langle 110\rangle$ and $<100>$ respectively. (for line scan comparison the AFM images has been normalized to crown region) 
sidewalls of neighboring pits. Close inspection of high-resolution images reveals that the two corners facing adjacent crowns are truncated, fostering the appearance of elongation.

In all the pattern wavelengths explored here, for pit-in-terrace morphologies, the QDs localize at the bottom of pits, as shown in Figure 6-23. Pit-in-terrace is a very well-studied pattern morphology for the growth of QDs and many studies have shown that the QDs preferentially self-assemble at the bottom of the pit as surface-minimization and elastic relaxation drives the site selection process. ${ }^{105,113-115,134}$ At finer lengthscales, when adjacent pit edges approach each other, the growth surface can no longer be viewed as discrete pits surrounded by flat (001) terraces but becomes a continuously height modulated, quasisinusoidal profile, as discussed in Section 6.4. As discussed in Chapter 5, theoretical studies suggest that interactions between the substrate pattern wavelength $\left(\lambda_{\mathrm{p}}\right)$ and the intrinsic lengthscale or the instability wavelength of the alloy ( $\left.\lambda_{\mathrm{ATGS}}\right)$ becomes important for such profiles during the early stages of QD self-assembly process.

The instability wavelength is given by $\lambda_{\mathrm{ATGS}}=(4 \pi / 3)^{*}\left(\gamma / \mathrm{M}^{2}\right)$, see Chapter 1 , where $\gamma$ is the surface energy, $\mathrm{M}$ is the biaxial modulus and $\varepsilon$ is the misfit strain. ${ }^{31-33} \mathrm{For}^{\mathrm{Si}} \mathrm{Si}_{0.5} \mathrm{Ge}_{0.5}$, using $\mathrm{M}=161.7 \mathrm{GPa},{ }^{47} \gamma=65.5 \mathrm{meV} / \AA^{2},{ }^{48,49}$ and $\varepsilon=-0.0195$, we obtain $\lambda_{\mathrm{ATGS}} \approx 72 \mathrm{~nm}$. In our case, for $\lambda_{\mathrm{p}}=150 \mathrm{~nm} \approx 2 * \lambda_{\mathrm{ATGS}}$, when the pattern morphology changes from pit-interrace to quasi-sinusoid, the island localization changes from pit-bottom to saddle region respectively, see Figure 6-23.

In other studies, QDs were observed to self-assemble at the crown region of pit-patterned $\mathrm{Si}(001)$ substrates but a direct comparison with our work is difficult as most of the previous work do not have pattern pitch at comparable lengthscale to the $\lambda_{\text {ATGS. }}{ }^{101,108,109,135} \mathrm{We}$ previously observed the islands nucleating on the crowns with high pattern fidelity. ${ }^{102}$ In 
that work, patterning was performed using a focused ion beam and there can be concerns about low-level residual defectivity in the pits that can bias the adatom chemical potential. In this work, we have used EBL to avoid these issues. The growth of well-faceted QDs on patterned region is a good indicator of high quality epitaxial growth, see Figure 6-24. Grutzmacher et al. ${ }^{136}$ also studied the role of pattern morphology on QD site selection, albeit at only one pitch. They observed a very similar saddle-point site selection for pure Ge islands grown on pit-in-terrace patterns with 90x100 nm pitch, for a specific pit opening width. Their process actually created very fine grooves in the Si buffer that connect each pit site. This is different from the buffer morphology used here, where a quasi-sinusoidal surface is obtained, without grooving.

Figure 6-25 maps our results as a function of $\lambda_{\mathrm{p}}$ and $\mathrm{W}$, shown in absolute and in normalized values. Both $\mathrm{W}$ and $\lambda_{\mathrm{p}}$ were measured in separate samples where only the $\mathrm{Si}$ buffer was grown. The plots include results for both pit-in-terrace and quasi-sinusoidal pattern profiles. Throughout the patterned region, the maximum pit sidewall angle ranges from about $15-25^{\circ}$, depending on $\mathrm{W}$ and $\lambda_{\mathrm{p}}$. In principle, a phase space for site selection of epitaxial QDs of fixed composition on sinusoidal patterns will be a function of at least three characteristic variables (which can be appropriately scaled): the pattern wavelength, the film thickness, and some characteristic of the vertical:lateral aspect ratio of the pattern. The phase space established theoretically by Aqua, et al. ${ }^{39,120}$ is an explicit function of the first two variables, although in their model aspect ratio is also varied. ${ }^{120,137}$ In the current work, the film thickness is kept constant while we instead vary the pit opening width. Since the pit depth is constant, this changes the aspect ratio in both the pit-in-terrace and the quasi- 
sinusoidal transition regions. Of course, in the pit-in-terrace regions of the pattern fields, comparison to Aqua and Xu's predictions is inappropriate.
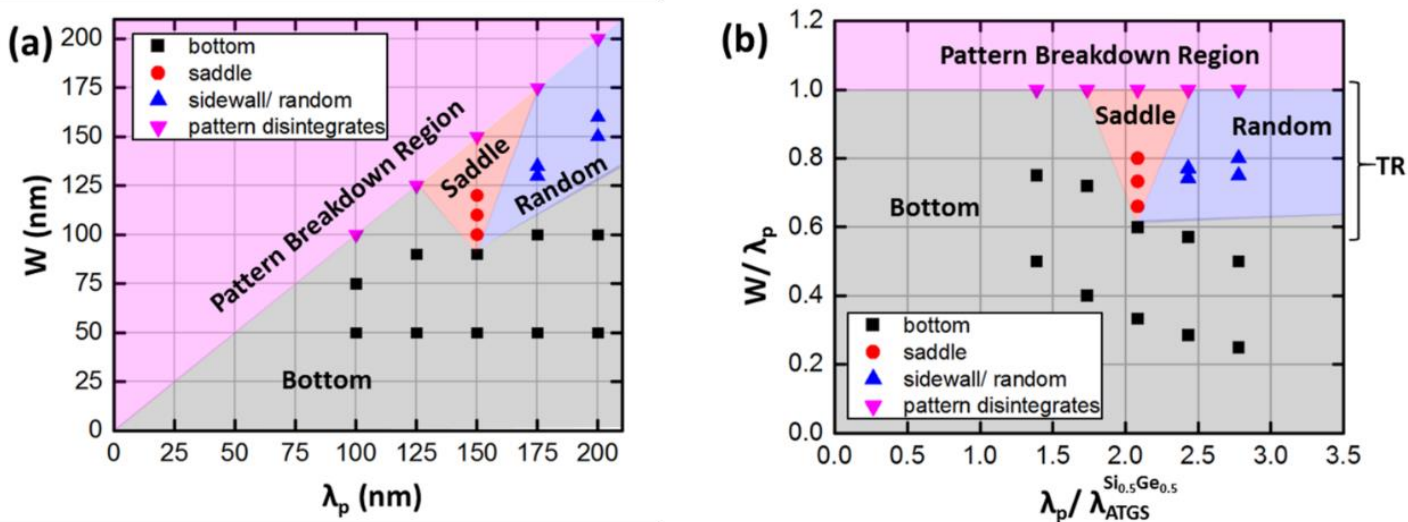

Figure 6-25: Maps of the observed QD site preference as a function of the pattern pitch $\left(\lambda_{\mathrm{p}}\right)$ and the pit opening width (W) for $1.3 \mathrm{~nm} \mathrm{Si}_{0.5} \mathrm{Ge}_{0.5}$ alloy growth with $\mathrm{t}_{\text {anneal }}=5 \mathrm{~min}$ at 650 ${ }^{\circ} \mathrm{C}$ in (a) absolute scale and (b) normalized scale. In (b), the transition region (TR) is indicated.

Figure 6-25(b) shows that for W/ $\lambda_{\mathrm{p}}<0.6$, the $\mathrm{Si}_{0.50} \mathrm{Ge}_{0.50}$ QDs always form in the bottoms of the discrete pits. This occurs with high site-occupation fraction, i.e., there is little obvious coarsening between sites. More interesting is the extremely narrow region of the map over which QDs form in the saddle points, i.e., between pairs of adjacent dots along the $\langle 110\rangle$ nearest neighbor directions. This behavior is repeatable across at least three different samples. QDs are never observed to form on the crowns for the given alloy thickness of $1.3 \mathrm{~nm}$. At small $\lambda_{\mathrm{p}}$, as $\mathrm{W}$ increases the pattern breaks down due to lithographic constraints during RIE, while at larger $\lambda_{\mathrm{p}}$, where the imposed lengthscale significantly exceeds the intrinsic lengthscale, QDs form at random locations inside the pits, with more than one QD per pit.

Careful examination of AFM linescans in both the pit-in-terrace and transition regions suggests there may be some net accumulation of SiGe at the crowns, see Figure 6-24. In 
the Si buffer, the crown regions clearly retain regions of flat (001) terrace, until pattern breakdown. However, after $\mathrm{SiGe}$ growth, there is obvious rounding of the crown regions. There is no residual (001) terrace to within the lateral resolution of these scans; neither is there any indication of $\{105\}$ facets yet. Hence any structures forming here are below the size needed to form the $\{105\}$ facet. ${ }^{37}$ To fully confirm that there is nascent QD formation on the crowns as well will require careful cross-section transmission electron microscopy, which has not yet been obtained.

The results shown in Figure 6-25(b) nominally disagree with those of Aqua and $\mathrm{Xu}^{39,120}$. Our maps represent a 2D space that would intersect Aqua and Xu's map, see Figure 4-12, as a horizontal line at $\mathrm{H} / \mathrm{H}_{\mathrm{c}}=1$, parallel to the $\lambda_{\mathrm{p}} / \lambda_{\mathrm{ATGS}}$ axis. This would then predict the formation of QDs on the crown regions over the range $1.2<\lambda_{\mathrm{p}} / \lambda_{\mathrm{ATGS}}<3.3$. Aqua and $\mathrm{Xu}$ did not find a region wherein QDs formed on saddle points, while we have not observed crown nucleation at quasi-sinusoidal regions in this work. However, some differences may be critical to this comparison. In particular, the aspect ratios (sinusoidal amplitude to wavelength) used in our patterns are significantly larger than those used by Aqua and Xu, where the pattern slope considered were lower than $11^{\circ} .{ }^{120}$ Also, our patterns in the transition region are quasi-sinusoidal along the in-plane $<110>$ nearest-neighbor directions. However, along the in-plane $<100>$ directions, where the interdot distance is $\sqrt{2}$ larger, a region of flat (001) terrace is still retained. Obtaining completely sinusoidal surfaces is frustrated by faceting at larger $\lambda_{\mathrm{p}}$, and by pattern degradation at smaller $\lambda_{\mathrm{p}}$. More work is needed to overcome this limitation.

Our work here shows how SiGe QD site selection, which occurs during annealing of an initially conformal film, changes as the surface evolves from discrete pits to coupled 
(quasi-sinusoidal) pits. The formation of QD's at the saddle points, as shown in Figure 624(a) is unusual. Since the number of dots per area doubles relative to pit nucleation, the volume per QD is halved. Grutzmacher et al. ${ }^{136}$ have noted this behavior as well, and the fact that they observe similar site selection in pure Ge under a narrow range of conditions, in analogy to our observation in $\mathrm{Si}_{0.5} \mathrm{Ge}_{0.5}$, indicates this may be a universal behavior that still needs to be better understood. Increasing either the annealing time or the thickness of the alloy as discussed in Section 6.5 and 6.6, did result in the formation of QDs in saddle points in quasi-sinusoidal regions for larger pitch patterns $(175 \mathrm{~nm}$ and $200 \mathrm{~nm})$ but with reduced uniformity suggesting strain relaxation plays a critical role during the localization process.

Theories suggest preferred nucleation can occur at regions of maximum negative (pit) or positive (crown) curvature, while the saddle point contains both negative and positive curvature, although neither is a global maximum in magnitude. An interesting feature of the linescan in Figure 6-24(e) is that the $\{105\}$-faceted QDs appear to merge smoothly with the pit sidewall, which suggests a role of the local surface slopes in facilitating formation of low-energy $\{105\}$ facets associated with stable SiGe QDs prior to the dome transition. ${ }^{138,139}$

\subsection{Summary}

We sought to gain both a broader perspective on, and deeper understanding for, the directed self-assembly of heteroepitaxial $\mathrm{Si}_{0.5} \mathrm{Ge}_{0.5}$ QDs on patterned $\mathrm{Si}(001)$ substrates, as well as to lay out the best path forward for creating 3D quantum dot mesocrystals. Hence, we systematically investigated how QD site selection, morphology and size distributions 
depended on pattern wavelength, and annealing conditions and pattern morphology. Key findings include:

- Si buffer layer growth successfully smooths and heals residual roughness and damage in the patterned surface after RIE. As a result, our QDs form the proper sequence of morphological transitions, which serves as an indicator of the health of this process. Ultimately, however, more sensitive probes of residual defectivity should be employed, such as photoluminescence.

- The EBL dose during patterning along with buffer layer growth can be used to fine-tune the pattern morphology from pit-in-terrace to more sinusoidal-like. However, the deposition temperature and thickness is critical to prevent a complete flattening and wipeout of the patterned region.

- Growth of QDs on the pit-in-terrace morphology leads to well-ordered QDs centered in the pits over a wide range of pattern wavelength and growth conditions. The formation of QDs below the critical WL thickness in these patterned regions suggests that each pit acts as a material sink resulting in larger WL thickness inside the pit compared to the non-patterned region. The pits also protect the QDs from Ostwald ripening, at least until the QD overgrows the pit.

- However, for quasi-sinusoidal morphology, when $\lambda_{\mathrm{p}}=150 \mathrm{~nm} \approx 2 * \lambda_{\mathrm{ATGS}}$, QDs suddenly bifurcate and shift to form in every saddle point, with high uniformity in size and site occupancy. Increasing the alloy thickness or annealing time results in the promotion of QDs formation at saddle points for larger pitch patterns (175 nm and $200 \mathrm{~nm})$ as well but with reduced uniformity suggesting strain relaxation is the primary driving force for the 
site selection. The saddle point contains both negative and positive curvature which appears in facilitating low-energy $\{105\}$ facets formation during QD growth.

- Unlike regular growth where QDs are formed under constant deposition flux at higher temperatures, we have opted to deposit an alloy film at lower temperature ensuring an initially conformal film with the substrate pattern. The film was then annealed at higher temperature to promote the formation of QDs. This provides an additional degree of freedom to control the average QD size. Our experimental findings indicate that the growth kinetics for this approach is initially controlled by the WL consumption across the entire unit cell defined by the pattern itself, resulting in an initially linear growth rate dependent on the pitch. For longer anneal time the growth is simply limited by ripening (for smaller $\lambda_{\mathrm{p}}$ ) or by diffusion kinetics (larger $\lambda_{\mathrm{p}}$ ).

- We used secondary nucleation of QDs in between pits to determine the effective diffusion length during $650{ }^{\circ} \mathrm{C}$ anneals of the metastable wetting layer. This is much smaller than values reported in the literature, due to various factors including the use of Si-Ge alloys (rather than pure $\mathrm{Ge}$ ) and the (thermally activated) requirement to form adatoms via detachment from step edges.

- Using this process, we have not only formed arrays of pyramidal QDs, but ordered arrays of steeper-faceted, dome-shaped QDs as well. This may be important towards QDMC formation. Domes will propagate inhomogeneous elastic fields over a greater lateral and vertical extent, promoting both strain-biased vertical alignment of dots, and the formation of $2 \mathrm{D}$ conduction channels between the dots. 


\section{Chapter 7: Summary}

Throughout this dissertation, we discussed strategies to obtain structural ordering of Group-IV semiconductor material systems from nano-to-meso scale in order to fine tune their transport properties relevant to thermoelectric applications. Even though, transport measurements and zT evaluation of the material was not covered in this work, it served as a core motivation for our exploration to understand the fundamental growth science in ordered $\mathrm{Si}_{1-\mathrm{x}} \mathrm{Ge}_{\mathrm{x}}$ material system.

We first discussed the mono-layer scale chemical ordering observed in $\mathrm{Si}_{1-\mathrm{x}} \mathrm{Ge}_{\mathrm{x}}$ thin film alloys grown by $\mathrm{MBE}$ on $\mathrm{Si}(001)$ and $\mathrm{Ge}(001)$ substrates. We presented our detailed study on chemical ordering of $\mathrm{Si}_{1-\mathrm{x}} \mathrm{Ge}_{\mathrm{x}}$ alloys with a parametric study, where effects of strain, surface roughness, and growth parameter on order parameter during the alloy growth was investigated. The observed order parameter never exceeded 0.24 across a range of growth temperatures, film thicknesses, substrate miscuts, and substrate types ( $\mathrm{Si}$ vs. Ge). Claims in the literature of much higher order parameters could not be reproduced here, despite a survey over multiple growth parameters suggested to be important to ordering in the literature, and this was true in a conventional MBE as well as in our sputtering-based MBE. We feel this may be due to inaccurate calculations of the order parameter in the relatively few works where quantification was performed. The effect of equivalent spatial ordering variants on the calculated order parameter was explicitly considered here. We found that smoother films, with extensive (001) terrace areas, led to the highest degree of order. The sign and magnitude of the strain was not directly important to ordering, other than for helping determine the evolution of the surface roughness and relaxation. Ordering was observed to persist during extensive roughening and even nominal $\{105\}$ faceting, 
suggesting the facets are not fully rebounded at these levels of strain. Growth on deliberately miscut substrates did not change the average order parameter, but did change the relative volume fraction of the ordered spatial variants. A key outcome here was that the ordering was both difficult to obtain and characterize. Finally, previous calculations ${ }^{16}$ on how ordering affects thermal transport suggest that much higher order parameters are required in order to obtain a significant effect which needs to be considered for future work.

In the second half of the dissertation, we presented our comprehensive investigation on the directed self-assembly of Si-Ge alloy QDs on patterned $\mathrm{Si}(001)$ substrates with variable morphology. We discussed the effects of $\mathrm{Si}$ buffer layer growth on substrate pattern morphology along with the effects of critical growth parameters like annealing time, growth temperature, pattern orientation, pitch and alloy thickness during QD evolution.

Growth of QDs on the pit-in-terrace morphology was observed to form well-ordered QDs centered in the pits over a wide range of pattern wavelength and growth conditions. Our observation of the formation of QDs below the critical WL thickness in these patterned regions suggests that each pit acts as a material sink resulting in larger WL thickness inside the pit compared to the non-patterned region. Further, the pit sidewalls are found to protect the QDs from Ostwald ripening, at least until the QD overgrows the pit. However, for quasi-sinusoidal morphology, when $\lambda_{\mathrm{p}}=150 \mathrm{~nm} \approx 2 * \lambda_{\mathrm{ATGS}}$, QDs were observed to suddenly bifurcate and shift to form in every saddle point, with high uniformity in size and site occupancy. Increasing the alloy thickness or annealing time resulted in the promotion of QDs formation at saddle points for larger pitch patterns $(175 \mathrm{~nm}$ and $200 \mathrm{~nm})$ as well but with reduced uniformity suggesting strain relaxation is the primary driving force for the site selection. A key morphological feature observed for saddle point regions are that 
they contain both negative and positive curvature which appears in facilitating low-energy $\{105\}$ facets formation during QD growth.

For the formation of QDs in this work, we opted to deposit an alloy film at lower temperature ensuring an initially conformal film with the substrate pattern. The film was then annealed at higher temperature to promote the formation of QDs. This provides an additional degree of freedom to control the average QD size. Our experimental findings indicate that the growth kinetics for this approach is initially controlled by the WL consumption across the entire unit cell defined by the pattern itself, resulting in an initially linear growth rate dependent on the pitch. For longer anneal time the growth was observed to simply be limited by ripening (for smaller $\lambda_{\mathrm{p}}$ ) or by diffusion kinetics (larger $\lambda_{\mathrm{p}}$ ).

Here we have successfully demonstrated not only the synthesis of highly ordered 2D arrays of pyramidal QDs, but ordered arrays of steeper-faceted, dome-shaped QDs as well. This may be important towards the future work for the QDMC synthesis as domes will propagate inhomogeneous elastic fields over a greater lateral and vertical extent, promoting both strain-biased vertical alignment of dots, and the formation of $2 \mathrm{D}$ conduction channels between the dots. 


\section{References}

1. Slack, G. \& Rowe, D. CRC handbook of thermoelectrics. CRC, Boca Raton, FL (1995).

2. Snyder, G. J. \& Toberer, E. S. Complex thermoelectric materials. Nat. Mater. 7, 105-114 (2008).

3. Chang, C. W., Okawa, D., Majumdar, A. \& Zettl, A. Solid-state thermal rectifier. Science 314, 1121-4 (2006).

4. Wang, L. \& Li, B. Thermal Memory: A Storage of Phononic Information. Phys. Rev. Lett. 101, 267203 (2008).

5. Li, B., Wang, L. \& Casati, G. Negative differential thermal resistance and thermal transistor. Appl. Phys. Lett. 88, 143501 (2006).

6. Padture, N. P., Gell, M. \& Jordan, E. H. Thermal barrier coatings for gas-turbine engine applications. Science 296, 280-4 (2002).

7. Pop, E. Energy dissipation and transport in nanoscale devices. Nano Res. 3, 147169 (2010).

8. Dresselhaus, M. S. et al. New Directions for Low-Dimensional Thermoelectric Materials. Adv. Mater. 19, 1043-1053 (2007).

9. Furlong, R. R. \& Wahlquist, E. J. US space missions using radioisotope power systems. Nucl. News 42, 26-35 (1999).

10. Ourmazd, A. \& Bean, J. C. Observation of order-disorder transitions in strainedsemiconductor systems. Phys. Rev. Lett. 55, 765 (1985).

11. Müller, E., Nissen, H., Ospelt, M. \& Känel, H. von. Chemical ordering and boundary structure in strained-layer Si-Ge superlattices. Phys. Rev. Lett. 63, 1819 (1989).

12. LeGoues, F., Kesan, V. \& Iyer, S. Long-range order in thick, unstrained Si $0.5 \mathrm{Ge}$ 0.5 epitaxial layers. Phys. Rev. Lett. 64, 40 (1990).

13. Kuan, T. S. \& Iyer, S. S. Strain relaxation and ordering in SiGe layers grown on (100), (111), and (110) Si surfaces by molecular-beam epitaxy. Appl. Phys. Lett. 59, 2242 (1991).

14. Jesson, D. E. et al. Interplay between evolving surface morphology, atomic-scale growth modes, and ordering during SixGe1-x epitaxy. Phys. Rev. Lett. 70, 22932296 (1993).

15. Araki, T., Fujimura, N., Ito, T., Wakahara, a. \& Sasaki, a. Structural characterization of ordered $\mathrm{SiGe}$ films grown on $\mathrm{Ge}(100)$ and $\mathrm{Si}(100)$ substrates. $J$. Appl. Phys. 80, 3804 (1996). 
16. Duda, J. C., English, T. S., Jordan, D. A., Norris, P. M. \& Soffa, W. A. Reducing thermal conductivity of binary alloys below the alloy limit via chemical ordering. J. Phys. Condens. Matter 23, 205401 (2011).

17. Duda, J. C., English, T. S., Jordan, D. A., Norris, P. M. \& Soffa, W. A. Controlling Thermal Conductivity of Alloys via Atomic Ordering. J. Heat Transfer 134, 14501 (2012).

18. Banhart, J. \& Czycholl, G. Electrical conductivity of long-range-ordered alloys. Europhys. Lett. 58, 264-270 (2002).

19. Fukatsu, S., Sunamura, H., Shiraki, Y. \& Komiyama, S. Phononless radiative recombination of indirect excitons in a Si/Ge type-II quantum dot. Appl. Phys. Lett. 71, 258-260 (1997).

20. Pernot, G. et al. Precise control of thermal conductivity at the nanoscale through individual phonon-scattering barriers. Nat. Mater. 9, 491-495 (2010).

21. Chen, P. et al. Thermal transport through short-period SiGe nanodot superlattices. J. Appl. Phys. 115, 44312 (2014).

22. Majumdar, A. Thermoelectricity in Semiconductor Nanostructures. Science (80-. ). 303, 777-778 (2004).

23. Wang, X. W. et al. Enhanced thermoelectric figure of merit in nanostructured ntype silicon germanium bulk alloy. Appl. Phys. Lett. 93, 193121 (2008).

24. Fromherz, T. \& Stangl, J. 3D SiGe quantum dot crystals: structural characterization and electonic coupling. Int. J. Mod. Phys. B 23, 2836-2841 (2009).

25. Fiedler, G. \& Kratzer, P. Theoretical prediction of improved figure-of-merit in Si/Ge quantum dot superlattices. New J. Phys. 15, (2013).

26. Mo, Y. W., Savage, D. E., Swartzentruber, B. S. \& Lagally, M. G. Kinetic pathway in Stranski-Krastanov growth of Ge on Si(001). Phys. Rev. Lett. 65, 1020-1023 (1990).

27. Floro, J. et al. Evolution of coherent islands in Si1-xGex/Si(001). Phys. Rev. B 59, 1990-1998 (1999).

28. Eaglesham, D.J.; Cerullo, M. Dislocation-Free Stransky-Krastanov Growth of Ge on Si(100). Phys. Rev. Lett. 64, 1943-1950 (1990).

29. Tersoff, J. Stress-induced layer-by-layer growth of Ge on Si(100). Phys. Rev. B 43, 9377-9380 (1991).

30. Rastelli, A. \& Von Känel, H. Surface evolution of faceted islands. Surf. Sci. 515, (2002).

31. Asaro, R. J. \& Tiller, W. A. Interface morphology development during stress 
corrosion cracking: Part I. Via surface diffusion. Metall. Trans. 3, 1789-1796 (1972).

32. Grinfeld, M. A. Instability of the interface between a nonhydrostatically stressed elastic body and a melt. Akad. Nauk SSSR 290, 1358-1363 (1986).

33. Srolovitz, D. J. On the stability of surfaces of stressed solids. Acta Metall. 37, 621625 (1989).

34. Lu, G. H. \& Liu, F. Towards quantitative understanding of formation and stability of Ge hut islands on Si(001). Phys. Rev. Lett. 94, 176103 (2005).

35. Ross, F., Tersoff, J. \& Tromp, R. Coarsening of Self-Assembled Ge Quantum Dots on Si(001). Phys. Rev. Lett. 80, 984-987 (1998).

36. Sutter, P. \& Lagally, M. G. Nucleationless Three-Dimensional Island Formation in Low-Misfit Heteroepitaxy. Phys. Rev. Lett. 84, 4637-4640 (2000).

37. Tersoff, J., Spencer, B., Rastelli, a. \& von Känel, H. Barrierless Formation and Faceting of SiGe Islands on Si(001). Phys. Rev. Lett. 89, 196104 (2002).

38. Jesson, D. E., Chen, K., Pennycook, S., Thundat, T. \& Warmack, R.

Morphological Evolution of Strained Films by Cooperative Nucleation. Phys. Rev. Lett. 77, 1330-1333 (1996).

39. Aqua, J. N. \& Xu, X. Growth of quantum dots on pit-patterns. Surf. Sci. 639, 2024 (2015).

40. Tromp, R., Ross, F. \& Reuter, M. Instability-driven SiGe island growth. Phys. Rev. Lett. 84, 4641-4 (2000).

41. Jesson, D. E., Pennycook, S. J., Baribeau, J. M. \& Houghton, D. C. Direct imaging of surface cusp evolution during strained-layer epitaxy and implications for strain relaxation. Phys. Rev. Lett. 71, 1744-1747 (1993).

42. Matthews, J. W. \& Blakeslee, A. E. Defects in epitaxial multilayers. J. Cryst. Growth 27, 118-125 (1974).

43. Yao, J. Y., Andersson, T. G. \& Dunlop, G. L. Structure of lattice-strained InxGa1$\mathrm{xAs} / \mathrm{GaAs}$ layers studied by transmission electron microscopy. Appl. Phys. Lett. 53, 1420-1422 (1988).

44. Gao, H. \& D. Nix, W. Surface Roughening of Heteroepitaxial Thin Films. Annu. Rev. Mater. Res. 29, 173-209 (1999).

45. Pidduck, A. J., Robbins, D. J., Cullis, A. G., Leong, W. Y. \& Pitt, A. M. Evolution of surface morphology and strain during SiGe epitaxy. Thin Solid Films 222, 78 84 (1992).

46. Cullis, A. G., Robbins, D. J., Pidduck, A. J. \& Smith, P. W. The characteristics of strain-modulated surface undulations formed upon epitaxial Si1-xGex alloy layers 
on Si. J. Cryst. Growth 123, 333-343 (1992).

47. Floro, J., Chason, E., Lee, S. R. \& Petersen, G. A. Biaxial moduli of coherent Si[sub 1-x]Ge[sub x] films on Si (001). Appl. Phys. Lett. 71, 1694 (1997).

48. Shklyaev, O., Beck, M., Asta, M., Miksis, M. \& Voorhees, P. Role of StrainDependent Surface Energies in Ge/Si(100) Island Formation. Phys. Rev. Lett. 94, $176102(2005)$.

49. Floro, J. \& Chason, E. Measuring Ge segregation by real-time stress monitoring during Si1 - xGex molecular beam epitaxy. Appl. Phys. Lett. 69, 3830-3832 (1996).

50. Biasiol, G. \& Sorba, L. Molecular beam epitaxy: principles and applications. Cryst. growth Mater. energy Prod. Energy-Saving Appl. 66-83 (2001). at $<$ http://www.chimica.unipd.it/gaetano.granozzi/privata/documentazione/insegnam enti/Fondamenti di Nanoscienza (A)/A15.MBE.pdf>

51. Vögeli, B., Zimmermann, S. \& von Känel, H. STM study of silicon (001) grown by magnetron sputter epitaxy. Thin Solid Films 318, (1998).

52. Sutter, P., Schick, I., Ernst, W. \& Sutter, E. Initial Surface Roughening in Ge/Si(001) Heteroepitaxy Driven by Step-Vacancy Line Interaction. Phys. Rev. Lett. 91, 176102 (2003).

53. Kudriavtsev, Y., Villegas, A. \& Godines, A. Calculation of the surface binding energy for ion sputtered particles. Appl. Surf. Sci. (2005). at <http://www.sciencedirect.com/science/article/pii/S0169433204009560>

54. Thompson, M. II. The energy spectrum of ejected atoms during the high energy sputtering of gold. Philos. Mag. (1968). at <http://www.tandfonline.com/doi/abs/10.1080/14786436808227358>

55. Handbook of Silicon Wafer Cleaning Technology, 2nd Edition. (William Andrew, 2008). at

<https://books.google.com/books?hl=en\&lr=\&id=5ORtHBrWyuQC\&pgis=1>

56. Meuris, M. et al. Implementation of the IMEC-Clean in advanced CMOS manufacturing. in 1999 IEEE International Symposium on Semiconductor Manufacturing Conference Proceedings (Cat No.99CH36314) 157-160 (IEEE, 1999). doi:10.1109/ISSM.1999.808761

57. Ishizaka, A. Low Temperature Surface Cleaning of Silicon and Its Application to Silicon MBE. J. Electrochem. Soc. 133, 666 (1986).

58. Thompson, P. E., Twigg, M. E., Godbey, D. J., Hobart, K. D. \& Simons, D. S. Low-temperature cleaning processes for Si molecular beam epitaxy. J. Vac. Sci. Technol. B J. Appl. Phys. J. Appl. Phys 11, 14311-8237 (1993).

59. Eaglesham, D. J., Higashi, G. S. \& Cerullo, M. $370{ }^{\circ} \mathrm{C}$ clean for Si molecular 
beam epitaxy using a HF dip. Cit. Appl. Phys. Lett. J. Vac. Sci. Technol. A J. Vac. Sci. Technol. B Appl. Phys. Lett. J. Appl. Phys. Phys. Lett 59, 1416-380 (1991).

60. Zhang, X.-J. et al. Thermal desorption of ultraviolet-ozone oxidized Ge(001) for substrate cleaning. J. Vac. Sci. Technol. A 11, 2553 (1993).

61. Whiteaker, K., Robinson, I., Van Nostrand, J. \& Cahill, D. Compositional ordering in SiGe alloy thin films. Phys. Rev. B 57, 12410-12420 (1998).

62. Rigaku. SmartLab Automatic X-Ray Diffractometer. Rigaku J. 23, 52-55 (2006).

63. Watkins, T., CAVIN, O. \& HUBBARD, C. Considerations Regarding the Alignment of Diffractometers for Residual Stress Analysis. Rigaku J. 23, 25-39 (2006).

64. Hartmann, J. M., Gallas, B., Zhang, J. \& Harris, J. J. Gas-source molecular beam epitaxy of SiGe virtual substrates: II. Strain relaxation and surface morphology. Semicond. Sci. Technol. 15, 370-377 (2000).

65. Zaumseil, P. A fast X-ray method to determine Ge content and relaxation of partly relaxed Si1-xGex layers on silicon substrates. Phys. status solidi 141, 155-161 (1994).

66. Dismukes, J., Ekstrom, L. \& Paff, R. Lattice Parameter and Density in Germanium-Silicon Alloys1. J. Phys. Chem. 68, (1964).

67. Hu, W. (Walter), Sarveswaran, K., Lieberman, M. \& Bernstein, G. H. Sub-10 nm electron beam lithography using cold development of poly(methylmethacrylate). $J$. Vac. Sci. Technol. B Microelectron. Nanom. Struct. 22, 1711 (2004).

68. Kassim, J. K. Ge1-x Mnx/Si (001) Heteroepitaxy: A study of how Mn Incorporates during Molecular Beam Epitaxial growth of Self Assembled Quantum Dots. (University of Virginia, 2012).

69. Petz, C. Epitaxial nanotemplates for strain modulated carrier confinement in Silicon. (University of Virginia, 2012).

70. Martins, J. \& Zunger, A. Stability of ordered bulk and epitaxial semiconductor alloys. Phys. Rev. Lett. 56, (1986).

71. Littlewood, P. Strain-induced ordering in silicon-germanium alloys. Phys. Rev. B 34, 2-5 (1986).

72. LeGoues, F., Kesan, V., Iyer, S., Tersoff, J. \& Tromp, R. Surface-stress-induced order in SiGe alloy films. Phys. Rev. Lett. 64, 2038 (1990).

73. Jesson, D. E., Pennycook, S. \& Baribeau, J.-M. Direct imaging of interfacial ordering in ultrathin $\left(\mathrm{Si}_{-}\{\mathrm{m}\} \mathrm{Ge}_{-}\{\mathrm{n}\}\right)_{-}\{\mathrm{p}\}$ superlattices. Phys. Rev. Lett. 66, (1991).

74. Tischler, J. Z., Budai, J. D., Jesson, D. E., Eres, G. \& Zschack, P. Ordered 
structures in SixGe1-x alloy thin films. Phys. Rev. B 51, 10947 (1995).

75. LeGoues, F. K., Tromp, R., Kesan, V. P. \& Tsang, J. C. Critical test of the structure of the ordered phase in epitaxially grown SixGe1-x films. Phys. Rev. B 47, (1993).

76. Muller, E., Nissen, H. U., Mader, K. A., Ospelt, M. \& Kanel, H. Von. Occurence of two ordered structures in Si-Ge systems. Philos. Mag. Lett. 64, 183-189 (1991).

77. Jesson, D. E., Pennycook, S., Baribeau, J. \& Houghton, D. C. Atomistic processes of surface segregation during Si-Ge MBE growth. Thin Solid Films 222, 98-103 (1992).

78. Kelires, P. \& Tersoff, J. Equilibrium Alloy Properties by Direct Simulation: Oscillatory Segregation at Si-Ge (100) 2x1 Surface. Phys. Rev. Lett. 63, 1164 (1989).

79. Kesan, V., LeGoues, F. \& Iyer, S. Influence of surface reconstructions and epitaxial-growth conditions on long-range order in Si 1-x Ge x alloys. Phys. Rev. B 46, 1576 (1992).

80. Chadi, D. Stabilities of single-layer and bilayer steps on Si (001) surfaces. Phys. Rev. Lett. 59, 1691-1694 (1987).

81. Araki, T., Fujimura, N. \& Ito, T. Mechanism for ordering in SiGe films with reconstructed surface. Appl. Phys. Lett. 71, 1174-1176 (1997).

82. Malachias, A. et al. X-ray study of atomic ordering in self-assembled Ge islands grown on Si(001). Phys. Rev. B 72, 1-9 (2005).

83. Malachias, a., Metzger, T. H., Stoffel, M., Schmidt, O. G. \& Holy, V. Composition and atomic ordering of $\mathrm{Ge} / \mathrm{Si}(001)$ wetting layers. Thin Solid Films 515, 5587-5592 (2007).

84. Richard, M.-I., Malachias, a, Schulli, T. \& Renaud, G. Ordered domain lateral location, symmetry and thermal stability in Ge:Si islands. Submitted 12108, (2013).

85. Richard, M.-I., Malachias, A., Schulli, T. \& Renaud, G. Ordered domain lateral location, symmetry and thermal stability in Ge:Si islands. Appl. Phys. Lett. 106, (2015).

86. Vantarakis, G., Remediakis, I. N. \& Kelires, P. C. Ordering Mechanisms in Epitaxial SiGe Nanoislands. Phys. Rev. Lett. 108, 176102 (2012).

87. Cullity, B. Elements of $x$-ray diffraction. (Prentice Hall, 2001).

88. Birkholz, M. Thin Film Analysis by X-Ray Scattering. (Wiley, 2006). at <https://books.google.com/books?hl=de\&lr=\&id=KMgQK8jB6zoC\&pgis=1>

89. Warren, B. X-ray diffraction. (Addison-Wesley Pub. Co., 1969). 
90. Ghatwai, P., Vetter, E., Hrdy, M. \& Soffa, W AFloro, J. Evolution of microstructure and magnetic properties in $\mathrm{Co}-\mathrm{Pt}$ alloys bracketing the eutectoid composition. J. Magn. Magn. Mater. 375, 87-95 (2015).

91. Xie, Y. et al. Semiconductor Surface Roughness: Dependence on Sign and Magnitude of Bulk Strain. Phys. Rev. Lett. 73, 3006-3009 (1994).

92. Lutterotti, L. \& Gialanella, S. X-ray diffraction characterization of heavily deformed metallic specimens. Acta Mater. 46, 101-110 (1998).

93. Hirth, J. P. \& Lothe, J. Theory of dislocations. (Wiley, 1982). at $<$ http://search.lib.virginia.edu/catalog/u181422>

94. Hashimoto, T., Morikawa, Y., Lagally, M. G. \& Et Al. Rebonded S B step model of Ge / Si ( 105 ) 1x2: A first-principles theoretical study. Surf. Sci. Lett. 513, 445 (2002).

95. Tersoff, J., Teichert, C. \& Lagally, M. Self-organization in growth of quantum dot superlattices. Phys. Rev. Lett. 76, 1675-1678 (1996).

96. Springholz, G. Self-Organized Growth of Three- Dimensional Quantum-Dot Crystals with fcc-Like Stacking and a Tunable Lattice Constant. Science (80-. ). 282, 734-737 (1998).

97. Zhu, J.-H., Brunner, K., Abstreiter, G. \& Brunner, ) K. Two-dimensional ordering of self-assembled $\mathrm{Ge}$ islands on vicinal $\mathrm{Si}(001)$ surfaces with regular ripples. Cit. Appl. Phys. Lett. Phys. Lett 73, (1998).

98. Teichert, C., Bean, J. C. \& Lagally, M. G. Self-organized nanostructures in Si 1-x Ge x films on Si(001). Appl. Phys. A Mater. Sci. Process. 67, 675-685 (1998).

99. Shchukin, V. A. \& Bimberg, D. Spontaneous ordering of nanostructures on crystal surfaces. Rev. Mod. Phys. 71, 1125-1171 (1999).

100. Baier, M. H., Watanabe, S., Pelucchi, E. \& Kapon, E. High uniformity of sitecontrolled pyramidal quantum dots grown on prepatterned substrates. Appl. Phys. Lett. 84, 1943-1945 (2004).

101. Pascale, A., Berbezier, I., Ronda, A. \& Kelires, P. C. Self-assembly and ordering mechanisms of Ge islands on prepatterned Si(001). Phys. Rev. B 77, 75311 (2008).

102. Duska, C. J. \& Floro, J. Highly uniform arrays of epitaxial Ge quantum dots with interdot spacing of $50 \mathrm{~nm}$. J. Mater. Res. 29, 2240-2249 (2014).

103. Hull, R. et al. Precision placement of heteroepitaxial semiconductor quantum dots. Mater. Sci. Eng. B Solid-State Mater. Adv. Technol. 101, 1-8 (2003).

104. Dais, C. et al. Ge quantum dot molecules and crystals: Preparation and properties. Surf. Sci. 601, 2787-2791 (2007).

105. Grydlik, M., Langer, G., Fromherz, T., Schäffler, F. \& Brehm, M. Recipes for the 
fabrication of strictly ordered Ge islands on pit-patterned $\mathrm{Si}(001)$ substrates. Nanotechnology 24, 105601 (2013).

106. Chen, G., Lichtenberger, H., Bauer, G., Jantsch, W. \& Schäffler, F. Initial stage of the two-dimensional to three-dimensional transition of a strained SiGe layer on a pit-patterned Si(001) template. Phys. Rev. B 74, 35302 (2006).

107. Kamins, T. I. \& Williams, R. S. Lithographic positioning of self-assembled Ge islands on Si(001). Appl. Phys. Lett. 71, 1201-1203 (1997).

108. Jin, G. et al. Controlled arrangement of self-organized Ge islands on patterned $\mathrm{Si}$ (001) substrates. Appl. Phys. Lett. 75, 2752-2754 (1999).

109. Zhong, Z., Halilovic, A., Mühlberger, M., Schäffler, F. \& Bauer, G. Ge island formation on stripe-patterned Si(001) substrates. Appl. Phys. Lett. 82, 445 (2003).

110. Schmidt, O. G. et al. Long-Range Ordered Lines of Self-Assembled Ge Islands on a Flat Si (001) Surface. Appl. Phys. Lett. 77, 4139 (2000).

111. Yoon, T. S. et al. Study of growth behaviour and microstructure of epitaxially grown self-assembled Ge quantum dots on nanometer-scale patterned $\mathrm{SiO} / \mathrm{Si}(001)$ substrates. Phys. Status Solidi B-Basic Solid State Phys. 246, 721724 (2009).

112. Yang, B., Liu, F. \& Lagally, M. G. Local strain-mediated chemical potential control of quantum dot self-organization in heteroepitaxy. Phys. Rev. Lett. 92, 25502 (2004).

113. Vastola, G. et al. How pit facet inclination drives heteroepitaxial island positioning on patterned substrates. Phys. Rev. B 84, 155415 (2011).

114. Vastola, G., Montalenti, F. \& Miglio, L. Understanding the elastic relaxation mechanisms of strain in Ge islands on pit-patterned $\mathrm{Si}(001)$ substrates. J. Phys. Condens. Matter 20, 454217 (2008).

115. Schülli, T. U. et al. Enhanced Relaxation and Intermixing in Ge Islands Grown on Pit-Patterned Si(001) Substrates. Phys. Rev. Lett. 102, 25502 (2009).

116. Dais, C. et al. SiGe quantum dot crystals with periods down to $35 \mathrm{~nm}$. Nanotechnology 26, 255302 (2015).

117. Grützmacher, D. et al. Three-dimensional Si/Ge quantum dot crystals. Nano Lett. 7, 3150-3156 (2007).

118. Zhang, J. J., Rastelli, a, Schmidt, O. G. \& Bauer, G. Role of the wetting layer for the SiGe Stranski-Krastanow island growth on planar and pit-patterned substrates. Semicond. Sci. Technol. 26, 14028 (2011).

119. Hu, H., Gao, H. J. \& Liu, F. Theory of directed nucleation of strained Islands on patterned substrates. Phys. Rev. Lett. 101, 216102 (2008). 
120. Aqua, J. N. \& Xu, X. Directed self-organization of quantum dots. Phys. Rev. E 90, 030402(R) (2014).

121. Duska, C. Towards Directed Self-Assembly of Quantum Dot Mesocrystals of $\mathrm{Ge} / \mathrm{Si}$ Using Focused Ion Beam Patterning. (University of Virginia, 2016).

122. Mohammad, M. A., Muhammad, M. \& Dew, S. K. Nanofabrication. (2012). doi:10.1007/978-3-7091-0424-8

123. Lee, Y.-H. Low voltage alternative for electron beam lithography. J. Vac. Sci. Technol. B Microelectron. Nanom. Struct. 10, 3094 (1992).

124. Kamp, M., Emmerling, M., Kuhn, S., Forchel, A. \& Emmerling, ) M. Nanolithography using a $100 \mathrm{kV}$ electron beam lithography system with a Schottky emitter. J. Vac. Sci. Technol. B J. Vac. Sci. Technol. B J. Vac. Sci. Technol. B J. Vac. Sci. Technol. B J. Vac. Sci. Technol. B J. Vac. Sci. Technol. B 171, (1999).

125. Chang, T. H. P. Proximity effect in electron-beam lithography. J. Vac. Sci. Technol. J. Vac. Sci. Technol. B J. Appl. Phys.346846 J. Vac. Sci. Technol. B J. Vac. Sci. Technol. J. Vac. Sci. Technol 12, 1271-2367 (1975).

126. Ocola, L. E. \& Stein, a. Effect of cold development on improvement in electronbeam nanopatterning resolution and line roughness. J. Vac. Sci. Technol. B Microelectron. Nanom. Struct. 24, 3061 (2006).

127. Jansen, H., Gardeniers, H. \& Boer, M. De. A survey on the reactive ion etching of silicon in microtechnology. 6, 14-28 (1996).

128. Zhang, J. J. et al. SiGe growth on patterned $\mathrm{Si}(001)$ substrates: Surface evolution and evidence of modified island coarsening. Appl. Phys. Lett. 91, 173115 (2007).

129. Grydlik, M. et al. Unrolling the evolution kinetics of ordered $\mathrm{SiGe}$ islands via $\mathrm{Ge}$ surface diffusion. Phys. Rev. B 88, 115311 (2013).

130. Barbato, A. \& Cavallotti, C. Challenges of introducing quantitative elementary reactions in multiscale models of thin film deposition. Phys. Status Solidi 247, 2127-2146 (2010).

131. Iguain, J. L. Dimer chain patterns during submonolayer growth of silicon on Si(100). J. Vac. Sci. Technol. A Vacuum, Surfaces, Film. 16, 3460 (1998).

132. Barnett, S., Rockett, A. \& Kaspi, R. Monte Carlo simulations of molecular beam epitaxy on Si (001) surfaces. J. Electrochem. ... 136, 1132-1137 (1989).

133. Dais, C. Templated Self-Assembly of SiGe Quantum Dots. (2009).

134. Zhong, Z. et al. Delayed Plastic Relaxation on Patterned Si Substrates: Coherent SiGe Pyramids with Dominant $\{111\}$ Facets. Phys. Rev. Lett. 98, 176102 (2007).

135. Szkutnik, P., Sgarlata, a., Nufris, S., Motta, N. \& Balzarotti, a. Real-time 
scanning tunneling microscopy observation of the evolution of Ge quantum dots on nanopatterned Si(001) surfaces. Phys. Rev. B 69, 201309 (2004).

136. Grützmacher, D., Dais, C., Zhang, L., Müller, E. \& Solak, H. H. Templated selforganization of SiGe quantum structures for nanoelectronics. Mater. Sci. Eng. C 27, 947-953 (2007).

137. Xu, X., Aqua, J. N. \& Frisch, T. Growth kinetics in a strained crystal film on a wavy patterned substrate. J. Phys. Condens. Matter 24, 45002 (2012).

138. Tomitori, M., Watanabe, K., Kobayashi, M. \& Nishikawa, O. STM study of the Ge growth mode on Si(001) substrates. Appl. Surf. Sci. 76-77, 322-328 (1994).

139. Medeiros-Ribeiro, G., Bratkovski, A. M., Kamins, T. I., Ohlberg, D. A. A. \& Williams, R. S. Shape Transition of Germanium Nanocrystals on a Silicon (001) Surface from Pyramids to Domes. Science 279, 353-355 (1998).

140. Berbezier, I. \& Ronda, a. SiGe nanostructures. Surf. Sci. Rep. 64, 47-98 (2009).

141. Paul, D. J. Si/SiGe heterostructures: from material and physics to devices and circuits. Semicond. Sci. Technol. 19, R75-R108 (2004).

142. Yasaka, M. X-ray thin-film measurment techniques V. X-ray reflectivity measurement. Rigaku J. 26, 1-9 (2010).

143. Zhong, Z. \& Bauer, G. Site-controlled and size-homogeneous Ge islands on prepatterned Si (001) substrates. Appl. Phys. Lett. 84, 1922-1924 (2004).

144. Biasiol, G., Gustafsson, a., Leifer, K. \& Kapon, E. Mechanisms of self-ordering in nonplanar epitaxy of semiconductor nanostructures. Phys. Rev. B 65, 1-15 (2002).

145. MicroChem. NANO PMMA and Copolymer. 8 (2001). 


\section{Appendix 1: Deposition rates and substrate temperature calibration}

\section{A1.1 Deposition rate calibration for DC magnetron sputter guns}

We used a Maxtek quartz crystal rate monitor (QCM) to calibrate the deposition rates of the $\mathrm{Si}$ and Ge sputter guns. QCM calibrates the deposition rates based on damped frequency. A quartz crystal is oscillated during deposition and frequency being damped due to deposited material (known density) is recorded over a period of time to calculate the thickness. The measured film thickness from QCM is given by,

$$
\mathrm{h}_{\text {film }}=\frac{\mathrm{N}_{\mathrm{q}} * \rho_{\mathrm{q}}}{\rho_{\text {film }}}\left(\frac{1}{\mathrm{f}_{0}}-\frac{1}{\mathrm{f}_{1}}\right)
$$

where $\mathrm{h}_{\mathrm{film}}$ is the film thickness, $\mathrm{N}_{\mathrm{q}}$ is the frequency constant for the bare quartz crystal, $\rho_{\mathrm{q}}$ is the density of the quartz, $\rho_{\mathrm{f}}$ is the density of the film and $\mathrm{f}_{\mathrm{o}}, \mathrm{f}_{1}$ are the frequencies before and after deposition.

The deposition rates of DC magnetron sputter guns in MBE can be controlled either by adjusting the output power or my physically changing the target-substrate distance which is done by adjusting the linear translation gun stage. The sputter targets used in our system are 0.25 " thick with 2 " diameter. The calibrated deposition rates for the sputter guns at various gun heights and output powers are given in Figure A1-1. The increase in deposition rate is linear with output power, but the effect of working distance is enhanced at larger powers. Figure A1-1 gives a plot of deposition rates vs. power at various gun heights where gun height is given in actual position of gun linear feed (i.e, higher the gun height shorter the distance between target and substrate) 
(a)

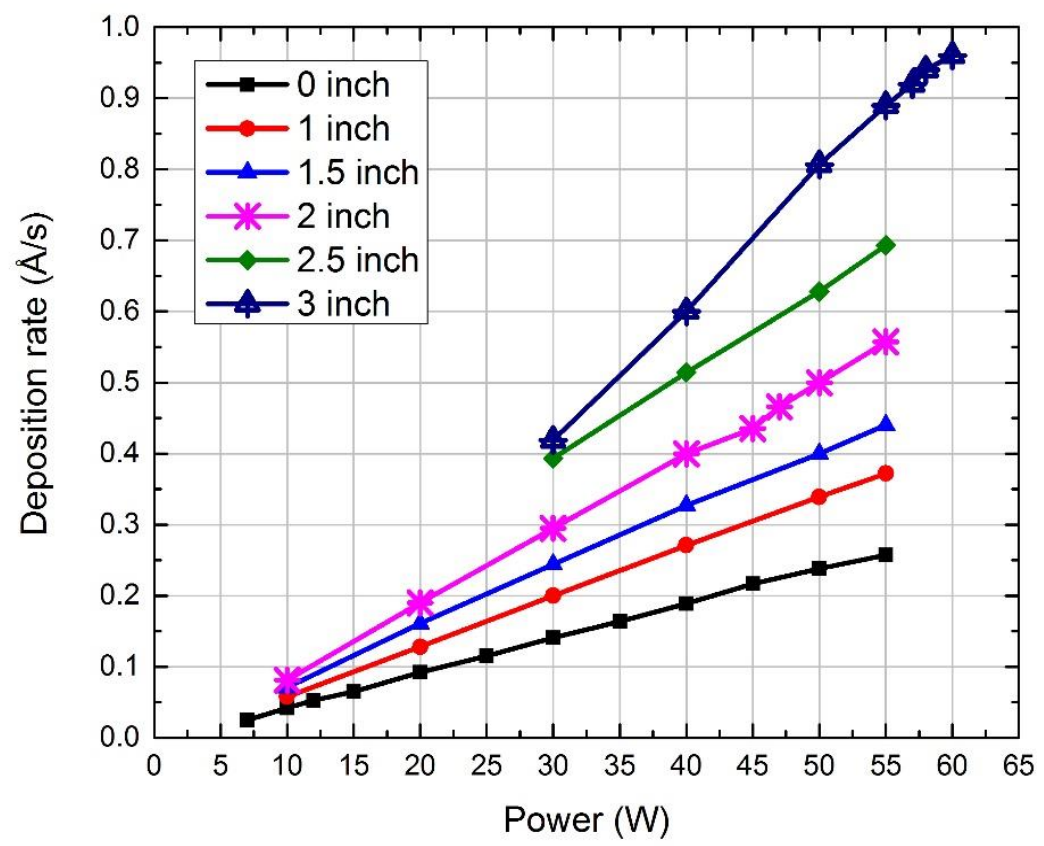

(b)

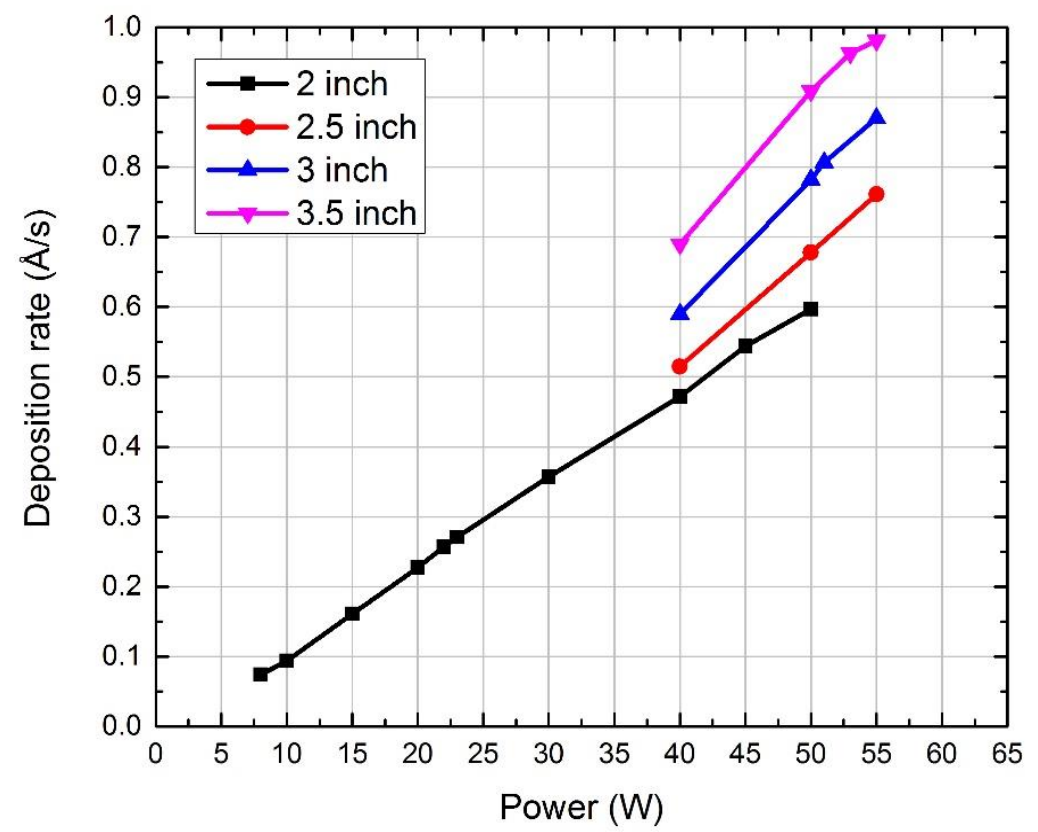

Figure A1-1: Deposition rates for (a) Si sputter target and (c) Ge sputter target. The calibration was performed at a chamber pressure of 4.75 mTorr of Ar. 


\section{A1.2 Temperature calibration}

For heating the substrate, the MBE is equipped with tungsten filament coiled around ceramic beads to uniformly heat the sample, see Figure A1-2. The sample during MBE operation is mounted over the heating filament. There is a K-type thermocouple (TC) at
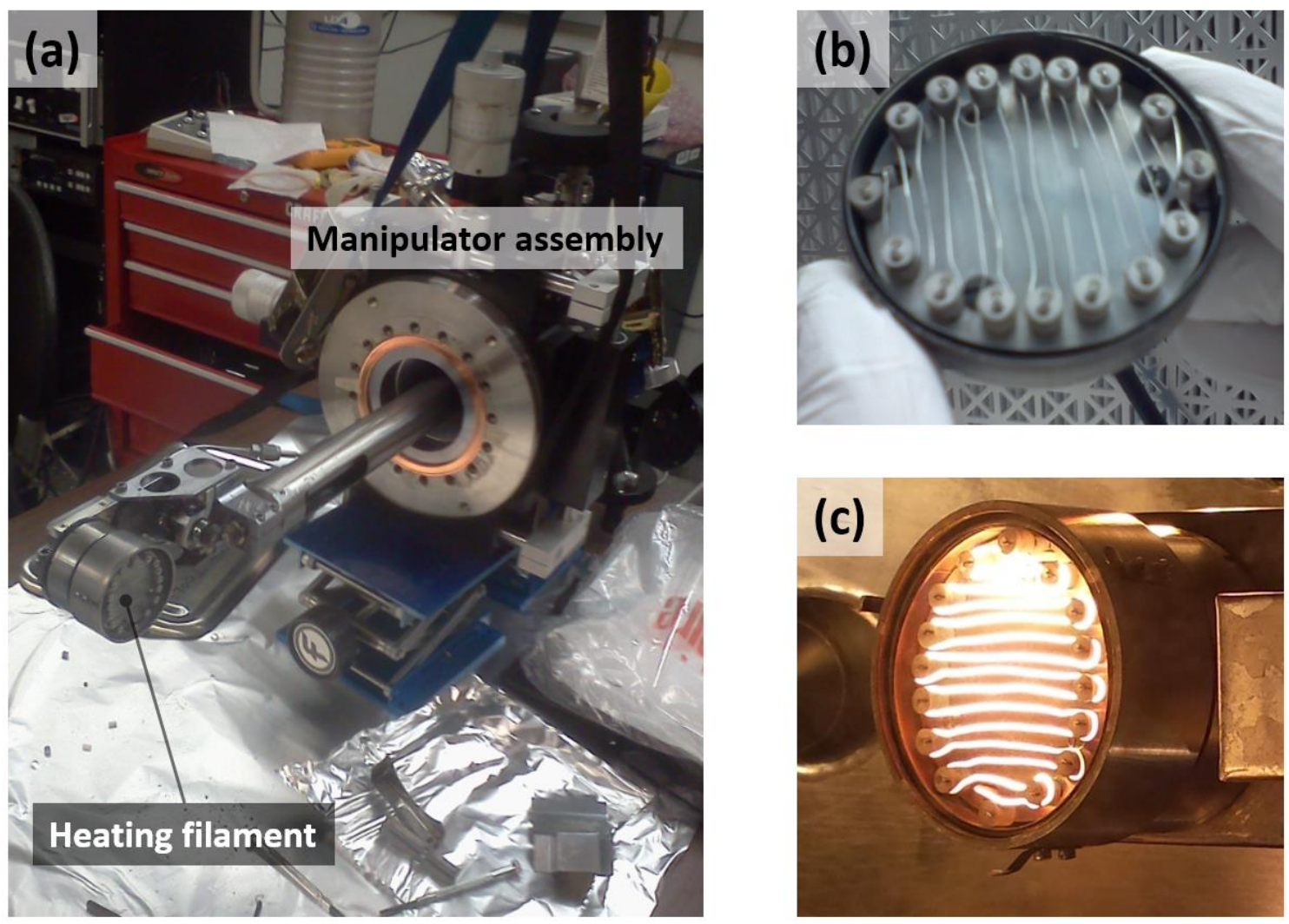

Figure A1-2: (a) Manipulator assembly installed with a 2 inch wafer handling system along with substrate heating assembly, (b) tungsten heating filament coiled around ceramic beads which is about 2 inch in diameter to heat the substrate uniformly, and (c) heating filament under operation at UHV condition.

the rear plate of the heating filament to measure the temperature but is always hotter than the substrate temperature itself. We do have a pyrometer which accurately measure the substrate temperature but is only rated to measure temperatures higher than $450{ }^{\circ} \mathrm{C}$. Therefore, a temperature calibration was performed to accurately monitor the substrate 
temperatures below $450{ }^{\circ} \mathrm{C}$. The temperature measured by TC and pyrometer holds a linear relation as shown in Figure A1-3, which was fitted to estimate the substrate temperatures below $450{ }^{\circ} \mathrm{C}$. The substrate temperature was also verified experimentally by clamping a TC in between two wafers and monitoring the steady state temperature with filament current.

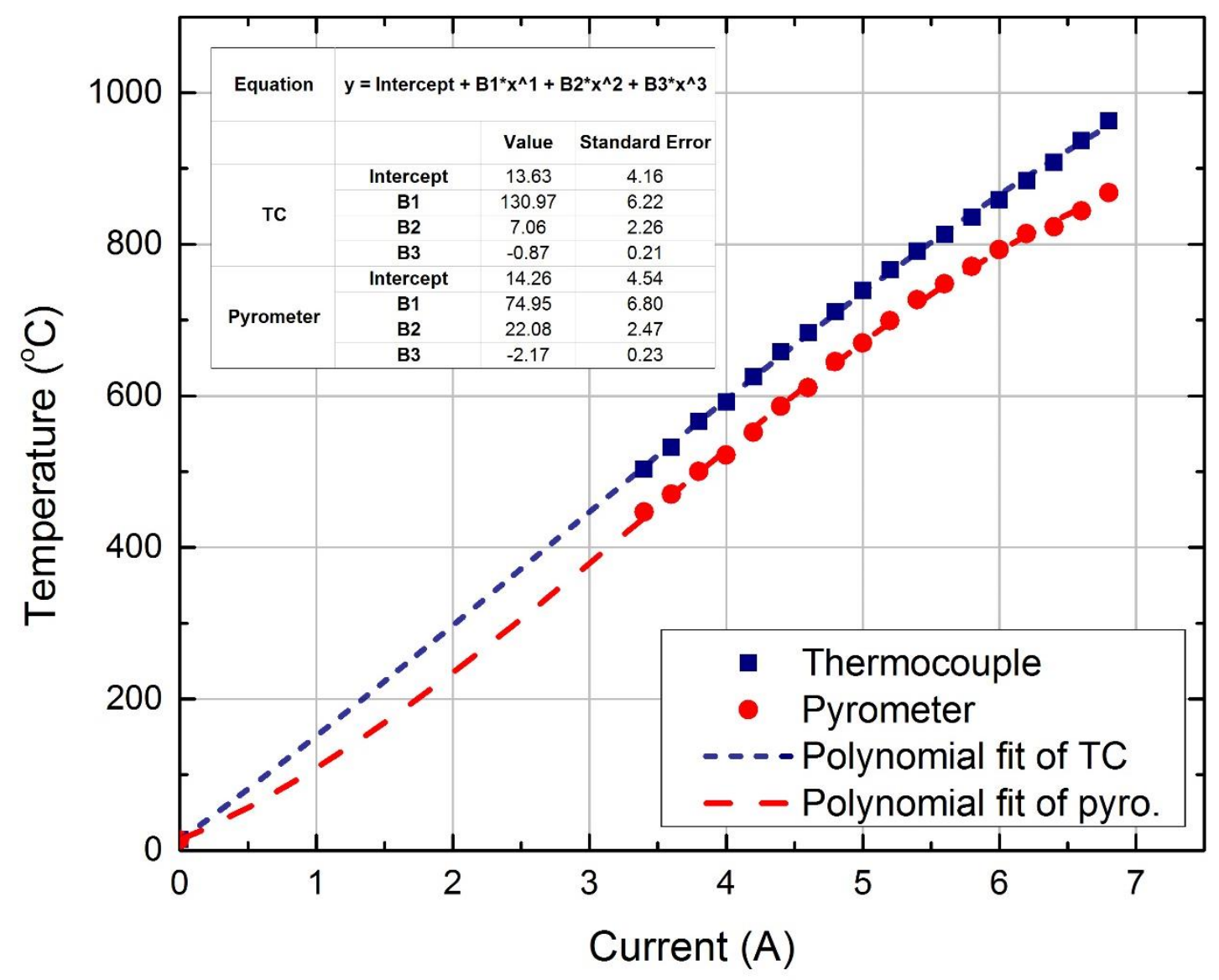

Figure A1-3: Temperature vs. Filament current plot 


\section{Appendix 2: IMEC-Shiraki Si cleaning process (Floro Group)}

Basic Components: PTFE Beakers and Dippers, Teflon-coated tweezers, $19 \mathrm{M} \Omega$ DI water, heated bath

Solutions: (solution stoichiometry is not critical)

IMEC: 4:1 Sulfuric : Peroxide $\left(\mathrm{H}_{2} \mathrm{SO}_{4}: \mathrm{H}_{2} \mathrm{O}_{2}\right)$; self-heated

Beaker should be $80 \%$ full

RCA-2: 4:1:1 Water : Hydrochloric : Peroxide $\left(\mathrm{H}_{2} \mathrm{O}: \mathrm{HCl}: \mathrm{H}_{2} \mathrm{O}_{2}\right) ; 80^{\circ} \mathrm{C}$

Beaker should be $75 \%$ full

OXIDATION: 2:4:1 Water : Hydrochloric : Peroxide $\left(\mathrm{H}_{2} \mathrm{O}: \mathrm{HCl}: \mathrm{H}_{2} \mathrm{O}_{2}\right) ; 80^{\circ} \mathrm{C}$

Beaker should be $20-25 \%$ full

\section{Notes:}

- Safety and Environment:

- Wear proper personal protection equipment (PPE)

- Keep the sash closed as much as possible

- Know the location of eyewashes and showers

- Only add acid to water, never the other way around

- HF IS INSIDIOUS - USE WITH EXTREME CAUTION

- Chemicals are never poured down the drain- always store waste in properly labeled containers, expeditiously have waste picked up when full

- Process Cleanliness:

- Dippers and tweezers are CLEAN - use only clean gloves to touch, rest only on clean lint-free cloths

- Beakers are dedicated to one specific solution and should never be used for anything else

- Avoid passing over any open beaker

- Do not let water run onto gloves and then into beakers

- Rinse beakers thoroughly before and after clean (at least $5 \mathrm{x}$ )

- Wipe down chemical bottles as needed to remove dust (before) or any acid drips (after) 


\section{Si wafer cleaning recipe:}

1. Pre-heat water bath to $80^{\circ} \mathrm{C}$, at least one hour prior to starting clean.

2. When bath has stabilized, mix RCA-2 and OXIDATION solutions (but don't add peroxide yet) and place in bath to heat.

3. Pre-rinse wafer in its dipper in beaker DI-1

4. Mix IMEC. Place wafer/ dipper into IMEC immediately. Etch for 5 min. (Rinse DI1 and BOE-1)

5. Place wafer/ dipper into full DI-1 and rinse for $3 \mathrm{~min}$. (Dip tweezers in IMEC for 10 sec, and rinse briefly in DI-1)

6. Fill BOE-1. Dip wafer into BOE for $20 \mathrm{sec}$. Should pull out dry.

7. Rinse in DI-1 for 2 min.

8. Add peroxide to RCA-2. When bubbling starts, place wafer/ dipper in. Etch for 2 min. (Rinse DI-2, BOE-1, BOE-2) Fill BOE-2.

9. Dip rinse in DI-2, then etch in BOE-2 for $20 \mathrm{sec}$. Dip rinse in DI-2 again.

10. Put wafer/ dipper back to RCA-2 for 2 more min. (Rinse DI-2)

11. Dip rinse in DI-2, then etch in BOE-2 for $20 \mathrm{sec}$. Dip rinse in DI-2 again.

12. Put wafer/ dipper back into RCA-2 for 2 more min. (Rinse DI-2 and BOE-3) Fill BOE-3.

13. Dip rinse in DI-2, then etch in BOE-3 for 20 sec.

14. Rinse in DI-2 for $5 \mathrm{~min}$.

15. Remove wafer from dipper, place in OXIDATION.

16. Add peroxide to OXIDATION. Warning - this is a violent solution!

17. After 5-6 min, add another shot of peroxide. (Rinse BOE-2, BOE-3, DI-2)

18. When OXIDATION solution has quenched, use tweezers to remove wafer, dip rinse, place back in dipper, rinse for 10 min in DI-2. Turn off water bath, remove RCA-2 and OXIDATION beakers.

19. Wafer can remain in running DI until ready to dry and load in laminar flow hood.

20. Dry wafer with blowing $\mathrm{N}_{2}$, inspect, mount, load and pump down.

21. After wafer has been loaded, allow solutions to cool for $30 \mathrm{~min}$ before pouring into waste. 


\section{Appendix 3: 2D slope distribution plot analysis}

Slope distribution plot enables to interactively plot the surface slope/facets occurring in the AFM data. The center of the plot corresponds to the horizontal facet, slope in $\mathrm{x}$-direction increases towards the left and right border and slope in y-direction increases towards up and bottom borders. For instance, if the surface morphology is smooth with minimal roughness mounds and faceted features the slope distribution plot will be ideally an "intensity dot" at the center of the plot denoting majority of the surface is plane with the horizontal facet. More details on the slope distribution plot analysis can be found in Gwyddion's software manual.

For our chemically ordered SiGe samples discussed in Chapter 3, Figure A3-1 shows the slope distribution plots for alloy samples with various thickness grown on $\mathrm{Ge}(001)$ substrate. An intense center dot in observed for all the samples, suggesting the surface is mostly smooth and coplanar with the growth (001) surface. However, comparing the 100 $\mathrm{nm}, 300 \mathrm{~nm}$ and $500 \mathrm{~nm}$ thick sample, the distribution appear to spread with the rise in thickness corroborating our analysis that roughness increases but at the same time overall (001) surface area decreases with the alloy thickness. Similar slope distribution plots are

presented for $\mathrm{SiGe}$ alloy films grown on $\mathrm{Si}(001)$ substrate in Figure A3-2. It is evident that the samples grown on $\mathrm{Si}(001)$ substrate are visibly more spread in the distribution plots indicating a much rougher surface morphology. However, there are distinct intensity points along $\langle 100\rangle$ in the plots for $100 \mathrm{~nm}$ and $300 \mathrm{~nm}$ thick samples at $11.3^{\circ}$ referring to the $\{105\}$ facets of the QDs present on the surface, see Figure A3-2(a) and (b). 


\section{(a) $100 \mathrm{~nm} \mathrm{SiGe/Ge}$}
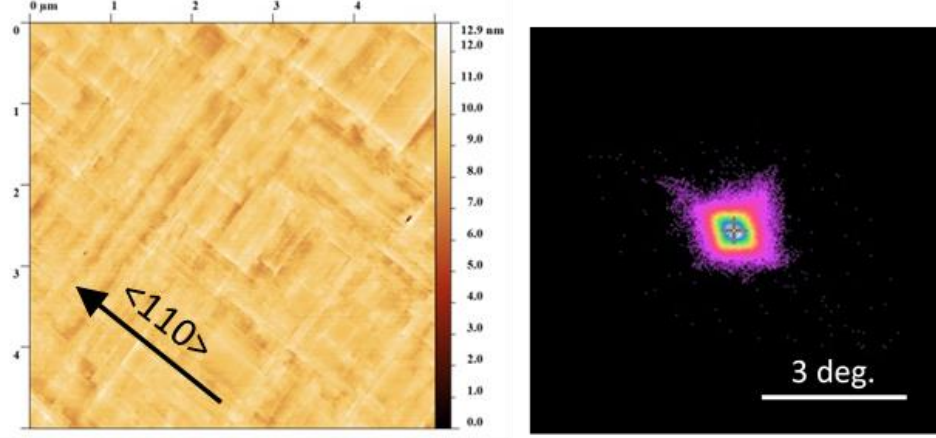

(b) $300 \mathrm{~nm} \mathrm{SiGe/Ge}$
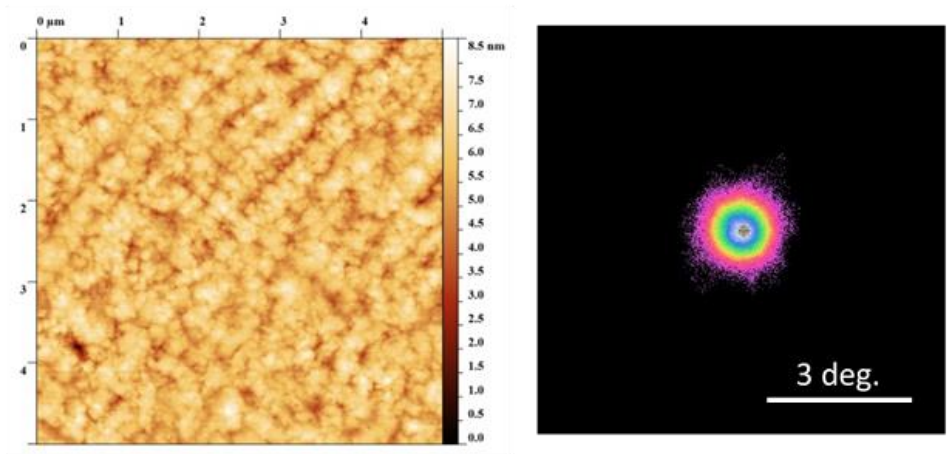

\section{(c) $500 \mathrm{~nm} \mathrm{SiGe/Ge}$}
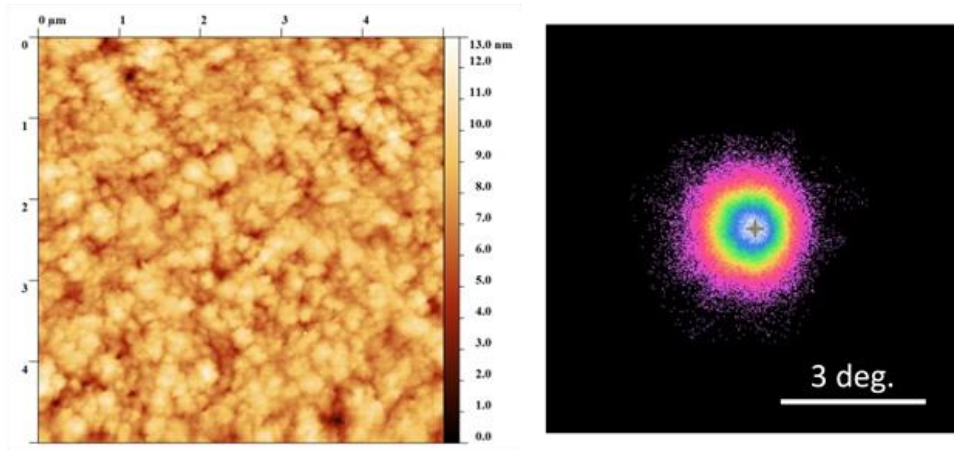

Figure A3-1: Slope distribution plots of $\mathrm{Si}_{0.5} \mathrm{Ge}_{0.5}$ alloys grown on $\mathrm{Ge}(001)$ with thickness (a) $100 \mathrm{~nm}$, (b) $300 \mathrm{~nm}$, and (c) $500 \mathrm{~nm}$. 
(a) $100 \mathrm{~nm} \mathrm{SiGe/Si}$

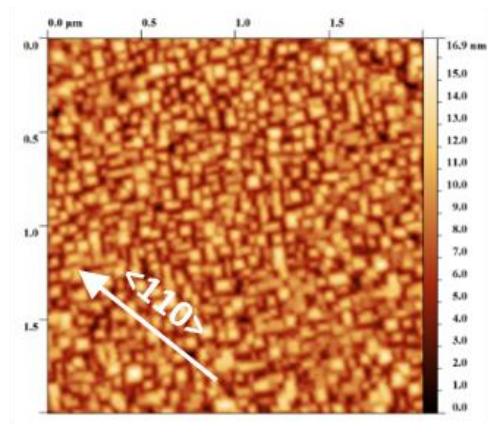

(b) $300 \mathrm{~nm} \mathrm{SiGe/Si}$
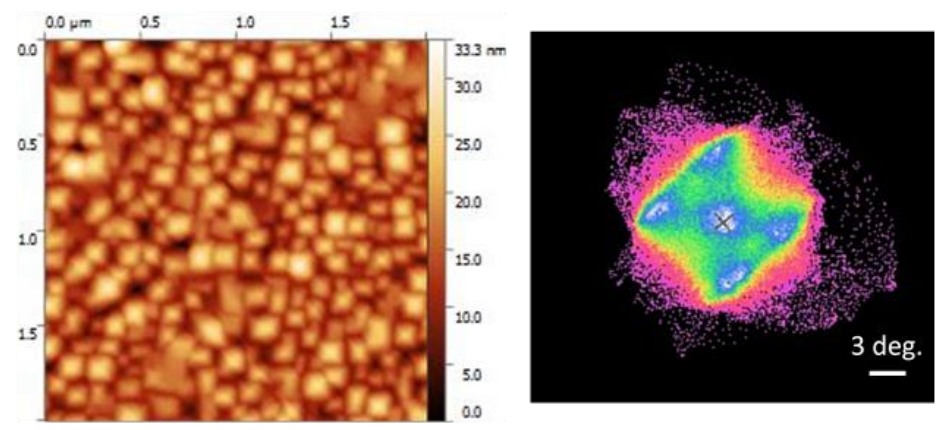

(c) $500 \mathrm{~nm} \mathrm{SiGe/Si}$
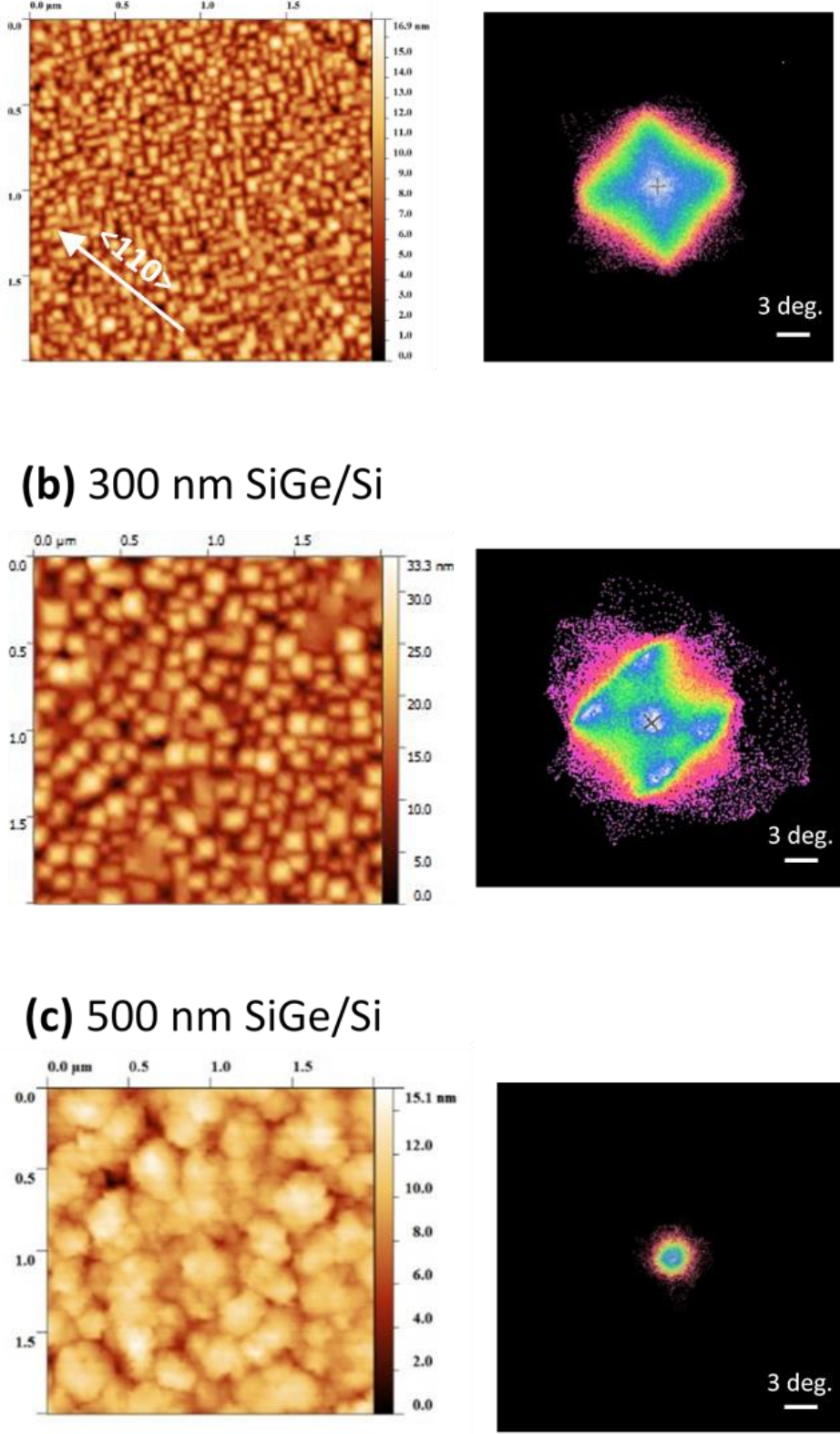

deg.

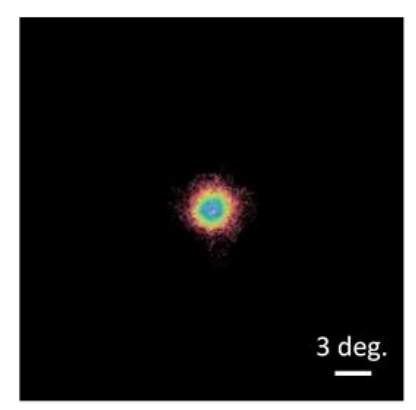

Figure A3-2: Slope distribution plots of $\mathrm{Si}_{0.5} \mathrm{Ge}_{0.5}$ alloys grown on $\mathrm{Si}(001)$ with thickness (a) $100 \mathrm{~nm}$, (b) $300 \mathrm{~nm}$, and (c) $500 \mathrm{~nm}$. 


\section{Appendix 4: Normality test: Probability plot}

A probability plot is a graphical method to test whether a dataset follows a given distribution. It shows a graph with an observed cumulative percentage on the $\mathrm{X}$ axis and an expected cumulative percentage on the $\mathrm{Y}$ axis. If all the data points are close to the reference line denoting a particular type of distribution (normal distribution is chosen for reference line in our work), we can say that the dataset follows the given distribution. (refer OriginPro software manual for more details). Probability plots are sometimes also referred to as Q-Q (Quantile-Quantile) plots. The only difference in that the Q-Q plot shows observed and expected value instead of percentages on axes.

For normality test of our data presented in Chapter 6, all the data were checked in OriginPro software by comparing the dataset to normal distribution reference line in the probability plot. Figure A4-1 presents the probability plots of the datasets for $100 \mathrm{~nm}$ pitch patterns with annealing time varying as $2 \mathrm{~min} 30 \mathrm{sec}, 5 \mathrm{~min}$ and $10 \mathrm{~min}$, refer Figure 6-7(a) in Chapter 6. Similar, tests were performed to confirm the normal distribution for all the datasets discussed in Chapter 6. 


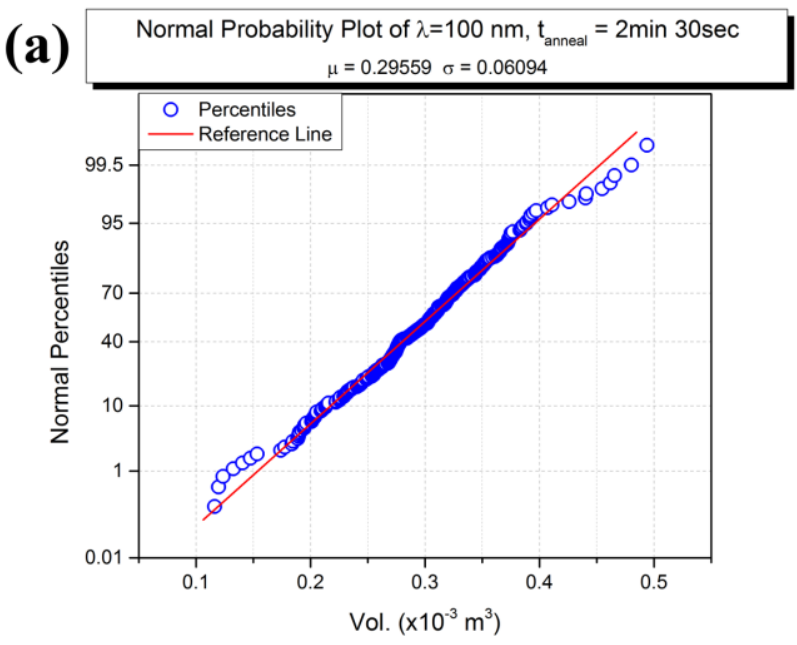

(b)
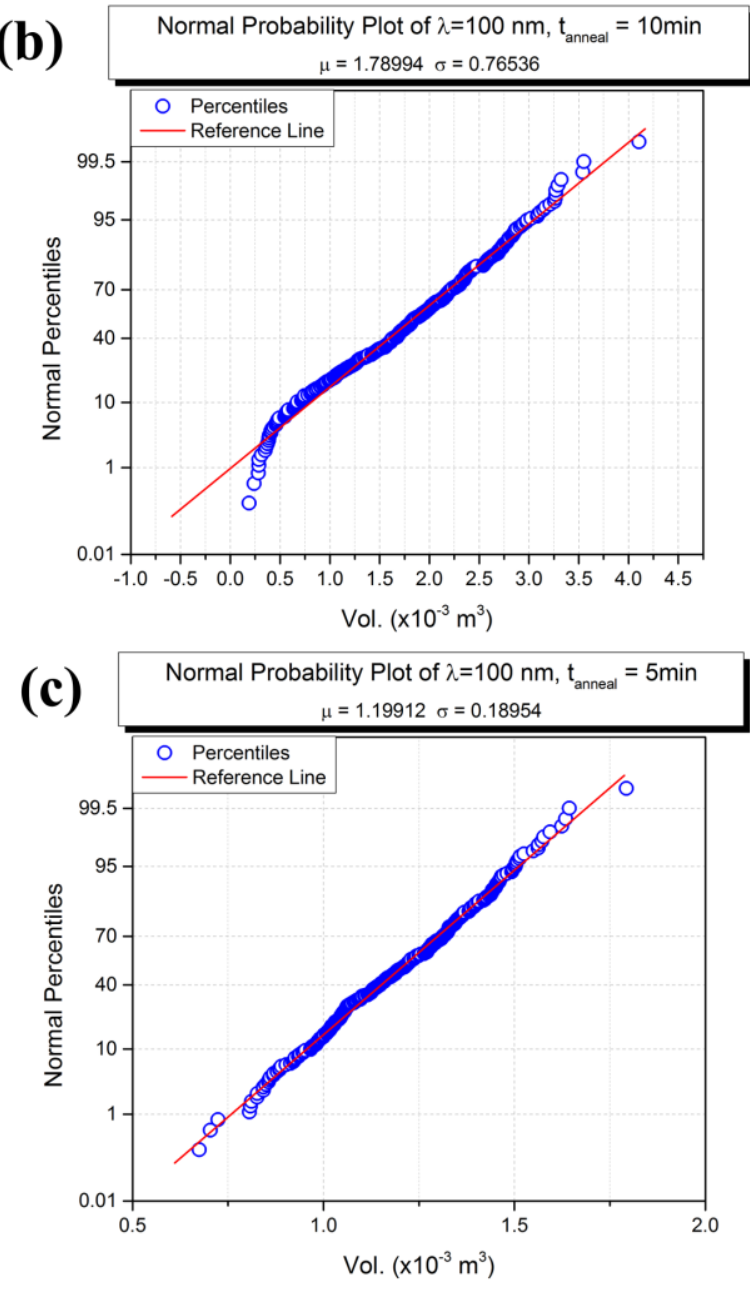

Figure A4-1: Probability plots comparing to the normal distribution reference line for three of the datasets discussed in Chapter $6.100 \mathrm{~nm}$ pitch pattern with a population of 300 points and anneal time of (a) $2 \mathrm{~min} 30 \mathrm{sec}$, (b) $5 \mathrm{~min}$, and (c) $10 \mathrm{~min}$. 